\title{
insoleresearch
}

\section{Evaluierung des Impulsprogramms FIT-IT und der Themeninitiative „IKT der Zukunft"}

Endbericht an das

Bundesministerium für Verkehr, Innovation und Technologie

Abteilung III//5

Radetzkystraße 2

1030 Wien

24. August 2018

Anton Geyer

Barbara Good

inspire research Beratungsgesellschaft m.b.H.

Lange Gasse 65/16, 1080 Wien

office@inspire-research.at 


\section{Kurzfassung}

Das Bundesministerium für Verkehr, Innovation und Technologie (BMVIT) förderte zwischen 2002 und 2012 mit dem Impulsprogramm FIT-IT ambitionierte kooperative Forschungs- und Entwicklungsvorhaben zu Informations- und Kommunikationstechnologien (IKT). Voraussetzung für die Förderung war die Zusammenarbeit von Unternehmen mit Forschungseinrichtungen. Durch die Vernetzung bestehender Kompetenzen in Wissenschaft und Forschung sollten mit der Förderung kritische Massen an österreichischer IKT-Forschung auf internationalem Spitzenniveau aufgebaut werden. Besondere Bedeutung hatte dabei die Etablierung neuer Kooperationen zwischen großen, international produzierenden Unternehmen und innovativen KMU.

Seit dem Jahr 2007 förderte das BMVIT darüber hinaus FuE-Projekte, die sich mit IKT-Lösungen zur Bewältigung des demographischen Wandels im Sinne der alternden Gesellschaft beschäftigen. Mit seinem nationalen Programm benefit beteiligt sich das BMVIT damit gleichzeitig auch am transnationalen Ambient Assisted Living (AAL) Programm, das nach Artikel 185 des Vertrags über die EU-Arbeitsweise von der Europäischen Kommission unterstützt wird. Das besondere Kennzeichen des Programms stellt die aktive Einbeziehung von Bedarfsträgern und Nutzern dar. In den Projekten sollen nutzerorientierte Geschäftsmodelle und innovative Wertschöpfungsketten erarbeitet werden. Damit will das Programm benefit frühzeitig Nischen für die heimische Wirtschaft schaffen und deren internationale Vermarktung fördern.

Als dritte Förderschiene innerhalb der IKT-Förderprogramme des BMVIT etablierte sich ebenfalls ab 2007 die österreichische Beteiligung an den Gemeinsamen Technologieinitiativen ARTEMIS (Embedded Systems) und ENIAC (Mikro- und Nanoelektronik). Mit diesen öffentlich-privaten Partnerschaften der europäischen Industrie, der Mitgliedsländer und der Europäischen Union sollte durch Zusammenführen der finanziellen Ressourcen eine gemeinsame Forschungsagenda umgesetzt werden, mit der die fragmentierte Forschungsanstrengungen der Mitgliedsländer überwunden und durch koordinierte Forschung die Wettbewerbsfähigkeit und zukünftiges Wachstum in Europa in den adressierten Technologiefeldern unterstützt werden. Nach sechs Jahren Laufzeit wurden die beiden Programme in der Gemeinsamen Technologieinitiative ECSEL zusammengeführt.

Im Jahr 2012 beendete das BMVIT das Impulsprogramm FIT-IT und richtete die nationale BMVIT-Förderung mit der Themeninitiative "IKT der Zukunft" neu aus. Das Förderthema Demographischer Wandel (benefit und AAL) und die Beteiligung an ECSEL-Projekten wurden mit der nationalen kooperativen FuE-Förderung (ehemals FIT-IT) in „IKT der Zukunft" zusammengeführt. Die nationale IKT-Förderung wurde an zwölf Förderthemen konzentriert und an BMVIT-relevanten Anwendungsfeldern gespiegelt. Damit einher ging eine Erweiterung des Instrumentenportfolios um strukturwirksame Förderinstrumente wie Leitprojekte und Stiftungsprofessuren im nationalen Förderschwerpunkt "IKT der Zukunft“.

Mit dieser Evaluierung sollten Design, Umsetzung und Wirkungen des Impulsprogramms FIT-IT ex-post analysiert und bewertet werden. Die Evaluierung diente gleichzeitig einer Interimsbewertung der aktuell laufenden Themeninitiative "IKT der Zukunft".

\section{Ergebnisse zum Impulsprogramm FIT-IT}

Insgesamt wurden über die Laufzeit des Impulsprogramms FIT-IT in den einzelnen Programmen und Programmlinien mehr als 1.000 Projektanträge mit einem beantragten Gesamtvolumen von 663 Mio. Euro eingereicht. Rund 67 \% der Anträge entfielen dabei auf die verschie- 
denen Programmlinien des Impulsprogramms FIT-IT. Etwa 9 \% der Anträge wurden für österreichische Beteiligungen an den Gemeinsamen Technologieinitiativen ARTEMIS und ENIAC eingereicht. Rund $24 \%$ der Anträge kamen für Projekte zum Demographischen Wandel. Mehr als 470 Anträge wurden genehmigt wobei der Barwert der Förderungen fast 165 Mio. Euro ausmachte. Mehr als 95 Mio. Euro entfielen dabei auf die nationale FIT-IT-Förderung. 25 Mio. Euro auf Projekte zum Demographischen Wandeln und rund 45 Mio. Euro auf ARTEMIS- und ENIAC-Projekte.

Die Ergebnisse der Evaluierung zeigen, dass es dem Impulsprogramm FIT-IT insgesamt sehr gut gelungen ist, die gesetzten Ziele zu erreichen. Ein sehr hoher Anteil von $81 \%$ der ehemals beteiligten wissenschaftlichen Partner und $67 \%$ der ehemals beteiligten Unternehmen sehen heute noch positive Nachwirkungen der geförderten Projekte in ihren Einrichtungen. Vor allem die Qualifizierung von Mitarbeiter/innen und der mit den Projekten erfolgte interne Know-how-Aufbau, aber auch die entstandenen Netzwerke und Kooperationen für die FuEZusammenarbeit im IKT-Bereich wurden als langfristiger Nutzen der FIT-IT Förderung genannt. Nach Einschätzung der Programmteilnehmer/innen hat FIT-IT maßgeblich zur Stärkung der Leistungs- und Wettbewerbsfähigkeit der österreichischen IKT-Forschung und zur Förderung der Kooperationen zwischen Wirtschaft und Wissenschaft beigetragen. Die Programmkonzeption, die Programmumsetzung und das Programmmanagement von FIT-IT werden von den ehemals Programmbeteiligten auch heute noch durchwegs als exzellent bewertet.

Die am Impulsprogramm FIT-IT beteiligten Unternehmen bewerten die wirtschaftlichen Erfolge und den Beitrag zur Steigerung ihrer Wettbewerbsfähigkeit durchwegs positiv. Fast die Hälfte (45\%) der an FIT-IT Projekten beteiligten Unternehmen berichteten über einen besseren Zugang zu Kunden und fast ein Viertel (24\%) über unmittelbare Umsatzsteigerungen bzw. verringerte Kosten aufgrund der Durchführung des Projekts. Der Hauptnutzen der Beteiligung an den FIT-IT Projekten wird aber im Aufbau von internem Know-how, der Verbesserung der Zusammenarbeit mit Forschungseinrichtungen und Unternehmen sowie der Basis für die Weiterentwicklung der Ergebnisse in FuE-Projekten und Kundenprojekten gesehen.

Die bibliometrischen Auswertungen zeigen eine intensive Publikationstätigkeit der wissenschaftlichen Partner, aber auch der beteiligten Unternehmen, während und nach der Laufzeit der FIT-IT Projekte. Die Auswertung der PATSTAT-Datenbank über die Patentanmeldungen der an den FIT-IT Projekten beteiligten Unternehmen zeigt eine sehr deutliche Zunahme der Patentierungsaktivitäten über die Laufzeit des FIT-IT-Programms, die mit einem deutlich steigenden Anteil der Bedeutung von Patenten in IKT-Technologiefeldern und einer Internationalisierung der Patentanmeldungen verbunden war.

Die ausgesprochen positive Einschätzung und Bewertung der Ergebnisse und Wirkungen des Impulsprogramms FIT-IT durch die ehemaligen Förderempfänger steht jedoch in einem Spannungsverhältnis mit den Ergebnissen einer im Zuge der Evaluation durchgeführten Sonderauswertung der FuE-Erhebung von Statistik Austria zur Entwicklung der internen FuEAusgaben und der FuE-Beschäftigen von geförderten und nicht-geförderten Unternehmen. Demnach kann aus den Daten der FuE-Erhebung nur für KMU und nur für die ersten Programmjahre von FIT-IT in der Gruppe der geförderten Unternehmen eine stärkere Entwicklung der internen FuE-Ausgaben und der Zahl FuE-Beschäftigen festgestellt werden. Die generelle Ausweitung der Angebote der direkten und indirekten (d. h. der steuerlichen) Forschungsförderung für Unternehmen seit Mitte der 2000er Jahre dürften hier die Auswertungsergebnisse beeinflusst haben. 


\section{Ergebnisse zur Themeninitiative IKT der Zukunft}

Durch die thematische Neuausrichtung des nationalen Förderschwerpunkts IKT der Zukunft mit der Festlegung von spezifischen IKT-Themenfeldern, Anwendungsfeldern und Querschnittszielen hat sich der fachlich-inhaltliche Charakter der Förderung im Vergleich zum Impulsprogramm FIT-IT deutlich verändert. Während die neuen IKT-Themenfelder bei den Programmbeteiligten insgesamt auf hohe Akzeptanz stoßen, trifft die nunmehrige verpflichtende Ausrichtung der Projekte an spezifischen Anwendungsfeldern nicht auf ausschließliche Zustimmung. Durch die Anwendungsfelder könnten stärker generisch ausgerichtete IKT-Projekte nicht mehr im Programm eingereicht werden. Außerdem wären die Alleinstellungsmerkmale des IKT-Programms im Vergleich zu anderen thematischen Förderprogrammen des BMVIT geringer geworden. Das IKT-Förderprogramm des BMVIT habe damit an eigenständigem Profil verloren, da anwendungsfeldorientierte IKT-Forschung auch in anderen thematischen Programmen adressiert werden würde.

Weiterhin als ausgezeichnet bewerten die Programmteilnehmer die Qualität der Programmdurchführung von IKT der Zukunft. Die Förderempfänger sind mit der Abwicklung des Programms ausgesprochen zufrieden. Hervorgehoben werden insbesondere die Informationsveranstaltungen zum Programm und zu neuen Ausschreibungen, die Einreichmodalitäten sowie die Kompetenz und Unterstützung durch die FFG-Mitarbeiter/innen bei administrativen und inhaltlichen Fragen.

Im nationalen Förderschwerpunkt IKT der Zukunft kam mit Leitprojekten auch ein neues Förderinstrument zum Einsatz, mit dem national vorhandenes Know-how zusammengeführt und durch Erarbeiten von modellhaften Lösungen für bedeutende gesellschaftliche Herausforderungen ein Sektor bzw. eine Branche spezifisch gestärkt werden soll. Leitprojekte dienen auch dazu, Bewusstsein für die jeweiligen Themenstellung zu schaffen und die nationale und internationale Sichtbarkeit von österreichischen Technologien zu erhöhen. Die bisher vorliegenden Erfahrungen zeigen, dass es dem Instrument Leitprojekt durchaus gelingt, die angestrebte Plattformfunktion wirksam auszufüllen und über das geförderte Projektkonsortium hinaus neue Partner für die Entwicklung von innovativen Lösungen zu mobilisieren.

Auf den nationalen Förderschwerpunkt IKT der Zukunft entfielen seit der Neugestaltung des Programms jedoch nur mehr ein Viertel der insgesamt für die Ausschreibungen im Zeitraum 2012 bis 2016 genehmigten Fördermittel. Mehr als die Hälfte der Mittel wurde für die Förderung österreichischer Partner in Projekten der Gemeinsamen Technologieinitiative ECSEL (bzw. deren Vorgängerinitiativen ARTEMIS und ENIAC) verwendet. Österreichische Projektpartner sind in der Hälfte aller in den ECSEL-Ausschreibungen der Jahre 2014 bis 2016 geförderten Projekte vertreten. Mit 29,5 Mio. Euro bewilligten EU-Beiträgen für diese Projekte liegt Österreich auf Rang 6 unter den beteiligten EU-Staaten. Nur die Niederlande und Belgien erhielten bezogen auf ihre Wirtschaftsleistung höhere EU-Beiträge als Österreich. Österreich hat sich damit sehr erfolgreich an ECSEL beteiligt. Gleichzeitig legen die Evaluierungsergebnisse nahe, dass trotz bereits gesunkener Förderintensitäten nach wie vor substanzielle Mitnahmeeffekte bei der Unternehmensförderung auftreten.

Der Förderschwerpunkt Demographischen Wandel ist unter den drei Förderschwerpunkten der Themeninitiative IKT der Zukunft nach wie vor die strukturell und organisatorisch ambitionierteste. Durch die Einbeziehung von nicht-klassischen Akteuren und dem Fokus auf Lösungen für (End-)Anwender/innen unterscheiden sich diese Projekte sehr deutlich von klassischen FuE-Projekten. Die Kombination von nationalen Ausschreibungen (benefit) und transnationalen Ausschreibungen (AAL) hat sich bewährt. Bezüglich der angestrebten Wirkungen und Ergebnisse des Förderschwerpunkts Demographischer Wandel stellt die Vermarktung 
von Lösungen für die beteiligten Unternehmen nach wie vor eine große Herausforderung dar. Die nicht-klassischen Partnerstrukturen machen auch die Aufrechterhaltung der Zusammenarbeit nach Projektende schwieriger als in klassischen kooperativen FuE-Projekten. Gleichzeitig wird aber auch eines der Hauptziele der Förderung erreicht, nämlich bei den Unternehmen das Verständnis über die Anforderungen und Bedürfnisse der Nutzer/innen und Endanwender/innen zu erhöhen.

\section{Empfehlungen zur Fortführung der Themeninitiative IKT der Zukunft}

Um die Abgrenzung des nationalen Förderschwerpunkts IKT der Zukunft zu anderen thematischen Programmen zu stärken, sollte die Förderung kooperativer FuE-Projekte stärker auf wissenschaftlich ambitionierte Forschungsvorhaben zwischen Forschungseinrichtungen und Unternehmen ausgerichtet werden. Dies kann weiterhin entlang der bestehenden Themenfelder des Programms erfolgen und auch primär Vorhaben unterstützen, die einen Beitrag zur Lösung von Herausforderungen in einem oder mehreren Anwendungsfeldern erwarten lassen, ohne diese jedoch unmittelbar ins Zentrum zu stellen. Kooperative FuE-Projekte der experimentellen Entwicklung sowie Vorhaben, die überwiegend durch die internen Forschungsleistungen der Unternehmen getragen werden, sollten hingegen bevorzugt in anderen (thematischen) Programmen gefördert werden. Durch eine stärkere Orientierung auf wissenschaftliche Grundlagen und besonders ambitionierte Forschungsfragen würden die Programmziele "Spitzentechnologien weiterentwickeln" und "Spitzenkräfte bereitstellen und gewinnen" noch besser unterstützt werden können. Die Konsortien der Projekte sollten durchaus strukturell anspruchsvoll sein, etwa in dem mehrere Forschungspartner und Unternehmen gemeinsam an komplexeren FuE-Fragen zusammenarbeiten. Die inhaltlichen Anforderungen und die Größe kooperativer FuE-Projekte in der Themeninitiative IKT der Zukunft sollte jedenfalls die Beschäftigung von Doktorand/inn/en ermöglichen, wobei ein entsprechendes Konzept bereits im Antrag gefordert werden könnte. Der Fokus von IKT der Zukunft auf wissenschaftlich besonders ambitionierte Forschung ließe sich auch sehr gut mit strukturell wirksamen Maßnahmen wie Stiftungsprofessuren kombinieren.

Bei der Programmumsetzung sollte das BMVIT auf einen Mix an Förderinstrumenten setzen, wobei neben kooperativen FuE-Projekten verstärkt auch Leitprojekte ausgeschrieben werden können. Voraussetzung ist allerdings, dass die Themen der Leitprojekte ausreichend inhaltlich spezifiziert werden können und ein Wettbewerb zwischen potenziellen Einreichern möglich ist. Sondierungsprojekte sollten weiterhin zur Unterstützung spezifischer Programmziele oder auch als Begleitmaßnahmen für Leitprojekten eingesetzt werden. Ausschließlich durch Unternehmen getragene Projekte, bzw. Vorhaben, in denen Forschungseinrichtungen nur in untergeordneter Rolle (z. B. im Unterauftrag) vertreten sind, sollten an das FFG-Basisprogramm verwiesen werden. Im Falle einer Förderung sollten derartige Projekte die gleichen Fördersätze erhalten, wie bei einer Förderung der Projekte im FFG-Basisprogramm (bzw. bei transnationalen Vorhaben als EUREKA-Projekt) gewährt werden.

Schließlich sollte das BMVIT die Programmmonitoringstrukturen weiter verbessern. Zur Unterstützung der Steuerung und kontinuierlichen Weiterentwicklung des Programms sollten Wirkungs- und Zielindikatoren festgelegt werden, die mit möglichst einfachen Mitteln, vergleichbar, regelmäßig bzw. zeitnahe erhoben werden können. Als zentrale Datenquelle bietet sich dazu die jährlich durchgeführte Befragung der Projektpartner abgeschlossener Projekte zum FFG Wirkungsmonitoring an. Das Nutzen der Daten aus dem FFG-Wirkungsmonitoring würde auch einen Vergleich der Förderwirkungen zwischen Programmen möglich machen. Voraussetzung dafür ist eine erhebungsjahrübergreifende Auswertung der Datensätze. 


\section{Executive Summary}

Between 2002 and 2012, the Austrian Federal Ministry of Transport, Innovation and Technology (BMVIT) funded ambitious collaborative R\&D projects in the field of information and communication technologies (ICT) under the umbrella of the FIT-IT programme. The FIT-IT scheme required collaboration between at least one (public) research partner (university or RTO) and at least one company for a consortium to receive funding. By joining the available competences of companies and research partners, the FIT-IT funding scheme aimed at creating critical mass of internationally competitive Austrian ICT-research capabilities. The FITIT programme also strove to create new collaborations between large, internationally active Austrian enterprises and innovative SMEs.

In 2007, BMVIT started to fund R\&D projects with a view to providing ICT-based solutions for mastering the challenges of demographic change and an aging society. With its national R\&D programme benefit, BMVIT contributes to the transnational Ambient Assisted Living (AAL) Joint Programme (since 2014: Active and Assisted Living Programme), which is co-funded by the European Commission in accordance with Article 185 of the Treaty on the Functioning of the European Union (TFEU). A special feature of this programme is the integration care organisations and end users in R\&D projects. Benefit projects develop user-oriented business models and innovative value chains that address challenges of the aging society. The programme helps establish market niches for domestic (service) suppliers and promote international commercialisation of solutions.

The third funding priority, which was established within the BMVIT ICT R\&D programme in 2007, supports Austrian participants in projects funded under the Joint Technology Initiatives (JTIS) ARTEMIS (embedded systems research) and ENIAC (micro- and nano-electronics research). These public-private partnerships formed between European industry, member states and the European Union pooled resources and implemented a strategic European research agenda in order to overcome fragmentation of national research efforts. The aim was to strengthen competitiveness and generate economic growth in various fields of technology in Europe. After six year of operation ARTEMIS and ENIAC merged to become the Joint Technology Initiative ECSEL.

In 2012, BMVIT renamed the ICT R\&D programme "thematic initiative ICT of the Future" and refocused national funding activities on 12 thematic R\&D priorities and specific fields of application in line with BMVIT's core policy competencies and responsibilities. As part of the redesign of the programme, BMVIT introduced Lead Projects and Endowed Chairs - new funding instruments aimed to stimulate structural change in the Austrian ICT R\&D landscape. The funding priority on Demographic Change (benefit, AAL) and the Austrian participation in ENIAC were integrated in the thematic initiative ICT of the Future.

This evaluation report analyses the design, implementation and impact of the FIT-IT programme 2002 - 2011. The evaluation also provides an assessment of the current funding programme ICT of the Future for the period $2012-2016$.

\section{Findings concerning the FIT-IT programme}

Over the ten-year FIT-IT programme period, applicants submitted more than 1,000 project proposals in response to the various calls in the three funding priorities. The total project budget of the submitted proposals amounted to more than $663 \mathrm{~m} €$. About $67 \%$ of the proposals concerned collaborative projects in the national FIT-IT programme lines. Approximately $9 \%$ of all applications were submitted in response to calls in relation to the Joint Technology Initiatives ARTEMIS and ENIAC, and $24 \%$ related to projects on demographic change. 
More than 470 proposals were accepted and received BMVIT funding, amounting to almost $165 \mathrm{~m} €$. National FIT-IT projects received about $95 \mathrm{~m} €$ funding. National and transnational projects on demographic change topics received financial support from BMVIT funds totaling 25 m€. The BMVIT contribution to Austrian participants in ARTEMIS and ENIAC projects amounted to $45 \mathrm{~m} €$.

The findings in this evaluation confirm that the FIT-IT programme succeeded in meeting most of its goals and objectives. A rather high share of $81 \%$ of scientific partners and $67 \%$ of company partners involved FIT-IT funded projects still notice long-term effects and impacts in their organisations. The projects mainly contributed to the qualification and training of (research) staff and to the creation of internal know-how and expertise. Further, the participants in the FIT-IT programme mentioned the establishment of new networks and R\&D collaborations in the field of ICTs as long-term benefits of the FIT-IT research projects. According to programme participants, FIT-IT contributed to strengthening the capacity and competitiveness of the Austrian ICT research community and significantly stimulated science-industry collaboration in the ICT area. The former programme participants still give high marks to the general FIT-IT design, the implementation of the programme and to the quality of the programme management.

Many companies involved in FIT-IT projects report significant economic effects and impacts regarding the projects' contribution to strengthening competitiveness. Almost half (45\%) of the company partners in FIT-IT projects report that access to clients has improved as result of the R\&D project, and almost a quarter of all companies (24\%) see a direct effect in relation to increasing turnover and / or reducing (production) costs. The most frequently mentioned benefits for companies, however, relate to building up internal know-how, improving R\&D networks with scientific partners and industry and laying the foundations for further (product / service) development in R\&D and client projects.

The bibliometric analysis of the publications by the lead collaborators of the scientific partners in FIT-IT projects points to a fairly strong publication activity during and after the duration of the projects, not only by scientific partners but in collaboration with company partners as well. The analysis of patent data covered in the PATSTAT data-base shows a significant increase in patent applications by companies funded in the FIT-IT programme over the FIT-IT programme period. This increase is particularly strong among patent applications in ICT-related technology fields and concerning international patent applications.

However, the very positive perception and assessment of the impacts of FIT-IT by the programme participants does not translate into better performance of the companies that received FIT-IT funding regarding their development of internal R\&D expenditures and internal R\&D personnel. Data analysis provided by the Austrian statistical office Statistik Austria shows that only SMEs and only companies funded in the early years of the FIT-IT programme increased their internal R\&D expenditures and the number of R\&D personnel at a higher rate than companies that did not participate in the FIT-IT programmes. The general expansion of direct and indirect (i. e. tax credit) R\&D support schemes for companies since the mid-2000s probably superimpose any effects of FIT-IT funding concerning these indicators.

\section{Findings concerning the FIT-IT programme}

As a consequence of the redesign of the national funding priority ICT of the Future, which was marked by a new set of specific R\&D themes, fields of application and some cross-cutting objectives the contents, projects differ considerably from those funded under the FIT-IT programme. Whilst the new set of R\&D themes is generally in line with the priorities and preferences of programme participants, it is the required alignment of these R\&D themes with 
specific fields of application which is seen as controversial. Some programme participants pointed out that due to the new focus on specific fields of application more generic R\&D projects became ineligible under the new funding requirements. Some programme stakeholders felt that through the changes in the design, the ICT R\&D programme lost its most distinguished feature, that is the funding of more generic ICT research. Many other thematic programmes fund ICT-related research in specific application areas (mobility, production, energy, etc.) whereas after the termination of the FIT-IT programme the ICT research community lacks a more generic ICT R\&D funding scheme.

However, administrative quality remained at a high level with the start of ICT for the Future. The programme participants still rate the quality of programme implementation and programme management very favorably. In particular, programme participants mention the range of available information about the programme and the public events on new calls as very positive features of programme implementation. To a high extent the participants are also satisfied with the proposal submission procedures and with the support they receive from FFG programme staff when they have administrative queries or questions regarding content.

In the funding priority of ICT of the future BMVIT introduced "lead projects" as a new funding instrument in order to pool available know-how of Austrian R\&D actors with a view to developing pilot applications and pilot solutions for key challenges in industry and society. Lead projects aim at strengthening specific industry sectors of companies. Furthermore, lead projects strive to raise awareness for certain themes or challenges and increase the visibility of innovative Austrian technology at national and international levels. The evidence available confirms that lead projects can be successful in creating national platforms and also attract new partners beyond the project consortia to join the development of innovative solutions.

Since the start of ICT of the Future, projects that are funded in the national collaborative R\&D scheme received only a quarter of the total funds available for the programme period 2012 to 2016. More than half of the financial aid was paid out to Austrian partners in project of the Joint Technology Initiative ECSEL (or to projects in the predecessor programmes ARTEMIS and ENIAC). Austrian organisations contributed to half of all projects selected and funded in the ECSEL calls 2014 to 2016. With $29.5 \mathrm{~m} €$ additional EU-funding Austria ranks $6^{\text {th }}$ under all ECSEL active member states. Only the Netherlands and Belgium received a higher share of EU-funds for ECSEL projects in relation to their total economic output. Hence, Austria's participation in ECSEL certainly has been a great success. At the same time evidence in this evaluation suggests that despite decreasing national funding intensity in ECSEL projects the deadweight losses with regard to company funding are still substantial.

The funding priority Demographic Change is the structurally and organisationally most ambitious part of the ICT of the Future programme. The involvement of various actors that are usually not seen as being R\&D active and the focus on funding the development of (end)user-oriented solutions for the elderly distinguish Demographic Change projects significantly from classical collaborative R\&D projects. The combination of national calls (benefit) and transnational calls (AAL) has proved adequate and useful. Concerning the goals and objectives of the funding priority Demographic Change there is still a lack of products and services that have been successfully introduced in the market. The non-classical structure of the project consortia also pose particular challenges, especially with regard to keeping up contact and continuing collaboration after the termination of the funded projects. Nevertheless, the funding priority Demographic Chance meets one of its key objectives: a high share of the companies involved in the projects report that their understanding of the requirements and needs of (end-)users has increased significantly through collaboration in the projects. 


\section{Recommendations}

In order to increase the unique features of the national funding priority ICT of the Future in relation to other thematic programmes, funding of projects should focus on scientifically ambitious, generic and cooperative R\&D between scientific partners and industry in the field of "industrial research". There is no need to change the current thematic R\&D priorities. The proposals should demonstrate the potential contributions of the research to certain application areas whilst not requiring dealing with specific applications as part of the research projects. Cooperative R\&D projects in the field of "experimental development" and projects that are predominantly carried out by companies alone should be directed towards other available thematic funding programmes. By focusing on generic and scientifically ambitious research questions the projects are expected to better support the programme goals "develop cutting-edge technology" and "provide and attract top personnel". The composition of the project consortia should be ambitious as well, by requiring the collaboration of several research partners and company partners in order to tackle more complex research questions. The scale and scope of the projects should allow PhD students to carry out their dissertations in the framework of the projects. The consortia should submit their concept for the involvement of PhD students as part of the application documents. The continued funding of Endowed Chairs would underline the focus of ICT of the Future on scientifically ambitious research.

BMVIT should continue to use a broad mix of funding instruments for the implementation of the programme. In addition to classical cooperative R\&D projects, BMVIT could increasingly call for applications for lead projects. However, lead projects require that the focus and the objectives of the projects can be laid down in sufficient detail in advance. There should also be competition between consortia in order to avoid moral hazard behaviour of applicants. Company-only applications and applications with research partners in subcontracting roles should be referred to the FFG Basisprogramm. In case there are convincing reasons to fund company-only projects in ICT of the Future, the funding intensity should not exceed the level of project funding in the FFG Basisprogramm (or for transnational projects in a EUREKA project).

Last but not least, BMVIT could improve the programme monitoring procedures. To support the governance and the continuous development of ICT of the Future, BMVIT should review the impact indicators laid down in the programme document. BMVIT should define indicators which can be collected in a timely manner, by simple means, regularly and that allow comparison over time and with other funding programmes. In this respect the annual FFG impact monitoring questionnaire survey, which surveys all project partners of FFG funded projects that ended four years before, could become the key data source for the information required on project level. Using FFG data would not only allow the monitoring of effects and impacts with relation to the goals and objectives of the programme but also make cross-programme comparisons possible. As a prerequisite, this would require the analysis of data collected over multiple years. 


\section{Inhaltsverzeichnis}

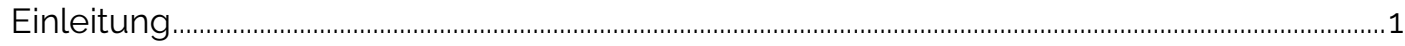

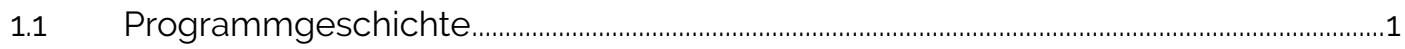

1.2 Ergebnisse bereits vorliegender Evaluierungen .................................................................... 7

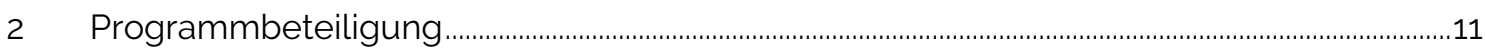

2.1 Beteiligung nach Programmlinien und Förderthemen ...................................................... 11

2.2 Beteiligung nach Teilnehmergruppen .......................................................................................16

2.3 Beteiligung von Klein- und Mittelunternehmen (KMU) .................................................. 22

2.4 Beteiligung erstmaliger Antragsteller/innen in FFG-Programmen........................... 25

2.5 Beteiligung von Frauen als technische Ansprechpersonen.........................................28

2.6 Wissenschaftliche Leistungen der Antragsteller und Projektauswahl.....................30

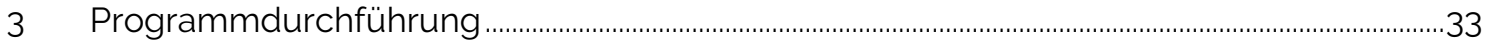

3.1 FuE-Zusammenarbeit und Vorerfahrungen mit den Partnern ................................... 33

3.2 Probleme bei der Projektdurchführung ……........................................................................... 38

3.3 Zufriedenheit der Förderempfänger mit der Programmdurchführung ...................40

3.4 Einschätzung der Entwicklung des Programmumfelds................................................ 44

$4 \quad$ Hinweise zum Programmkonzept und zu den Förderinstrumenten.....................................46

4.1 Inhaltliche Ausrichtung des Programms ...............................................................................46

4.2 Antragsverfahren und Berichtswesen ..................................................................................46

$4.3 \quad$ Förderquoten und Finanzierung ..................................................................................... 47

4.4 Leitprojekte ........................................................................................................................ 48

4.5 Förderschwerpunkt Demographischer Wandel ...................................................................49

4.6 Förderschwerpunkt Gemeinsame Technologieinitiative ECSEL ...............................50

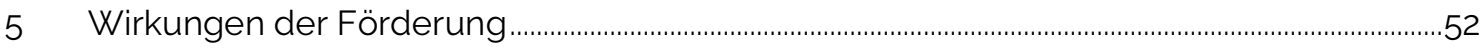

5.1 Langfristige Nachwirkungen der FIT-IT-Projektförderung............................................52

5.2 Nutzen und Auswirkungen von IKT der Zukunft-Projekten...........................................57

5.3 Wissenschaftliche Publikationen......................................................................................... 63

5.4 Patentanmeldungen der beteiligten Einrichtungen.......................................................69

5.5 Entwicklung von FuE-Ausgaben und FuE-Beschäftigung ........................................... 75

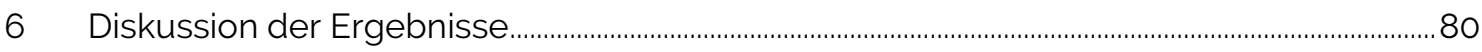

6.1 Konnten die Ziele des Impulsprogramms FIT-IT erreicht werden? ..........................80

6.2 Wie gut ist die Umsetzung von IKT der Zukunft bisher gelungen? .......................... 82

6.3 Wie erfolgreich ist die österreichische Beteiligung an der Gemeinsamen Technologieinitiative ECSEL?.

6.4 Welche Erfahrungen liegen für die Umsetzung des Förderschwerpunkts Demographischer Wandel vor?. 
$7 \quad$ Empfehlungen zur Fortführung von IKT der Zukunft. 91

7.1 Förderschwerpunkt IKT der Zukunft auf wissenschaftlich ambitionierte, generische FuE-Projekte ausrichten. 91

7.2 Durch Instrumentenmix die Strukturwirkung von Förderung stärken ......................91

7.3 Laufende FFG Erhebungen für das Monitoring des Programms nutzen............... 92

Anhang 1 Kennzahlen zur Entwicklung des IKT-Sektors in Österreich.......................................95

Anhang 2 Programmbeispiele zur IKT-Forschung in anderen Ländern...................................101

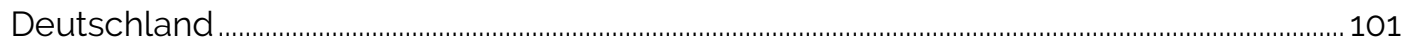

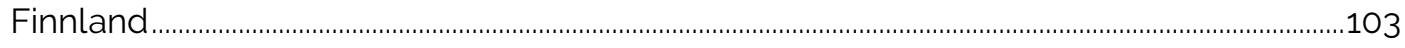

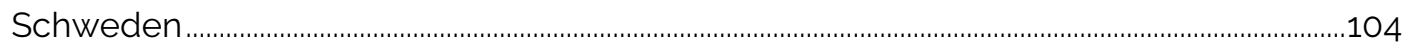

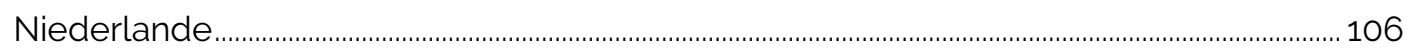

Fazit aus den internationalen Beispielen ...................................................................................... 108

Anhang 3 Online-Befragungen der Förderempfänger ....................................................................110

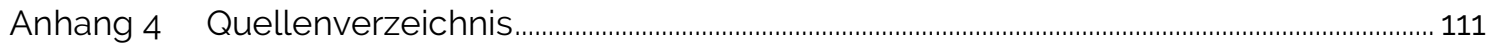




\section{$1 \quad$ Einleitung}

Gegenstand dieses Berichts ist die Ex-post Evaluierung des Impulsprogramms FIT-IT, das vom Bundesministeriums für Verkehr, Innovation und Technologie (BMVIT) in den Jahren 2002 bis 2012 durchgeführt wurde, und die Zwischenevaluierung der seit 2012 laufenden BMVIT-Themeninitiative "IKT der Zukunft" mit inren Programmlinien IKT der Zukunft, ECSEL und benefit / AAL. In der Ex-post Evaluierung des Impulsprogrammes FIT-IT wird im Sinne einer Gesamtbewertung der Programmdurchführung die Wirkung, Effektivität, Effizienz und Zielerreichung über die gesamte Programmlaufzeit dargestellt. Die Zwischenevaluierung der aktuellen Themeninitiative "IKT der Zukunft" dient vorrangig der Design- und Prozessevaluierung zur Qualitätsüberprüfung des Programmes. Zudem haben wir, soweit bereits möglich, Hinweise auf die Wirkungen der Förderung erhoben.

In diesem ersten Abschnitt beschreiben wir die Ziele, Schwerpunkte und Entwicklung der BMVIT IKT-FuE-Förderprogramme seit Start des Impulsprogramms FIT-IT. Daran anschlieBend stellen wird die Evaluierungsaufgabe dar und fassen die Ergebnisse bereits vorliegender früherer Evaluationen einzelner Programmteile zusammen.

\subsection{Programmgeschichte}

Das Bundesministerium für Verkehr, Innovation und Technologie (BMVIT) förderte ab 2002 mit dem Impulsprogramm FIT-IT ambitionierte kooperative Forschungs- und Entwicklungsvorhaben zu Informations- und Kommunikationstechnologien (IKT). Voraussetzung für die Förderung war die Zusammenarbeit von Unternehmen mit Forschungseinrichtungen. Durch die Vernetzung bestehender Kompetenzen in Wissenschaft und Forschung war es Ziel der Förderung, kritische Massen auf internationalem Spitzenniveau aufzubauen. Besondere Bedeutung hatte dabei die Etablierung neuer Kooperationen zwischen großen, international produzierenden Unternehmen und innovativen KMU.

\section{Programmziele}

Mit dem Impulsprogramm FIT-IT sollten insbesondere:

- radikal neue Informationstechnologie bis zum funktionsnachweisenden Forschungsprototyp am Standort Österreich entwickelt werden;

- die Wettbewerbsfähigkeit der österreichischen Forschung und Wirtschaft durch Kooperation von Forschung und Industrie, thematische Fokussierung und Bildung von Clustern gestärkt werden;

- qualifizierte Forscher/innen ausgebildet und kooperative anspruchsvolle Forschungsprojekte intensiviert werden;

- die europaweite und internationale Sichtbarkeit und Vernetzung der österreichischen Forscher/innen verbessert werden.

Im Mittelpunkt des Programms sollten visionäre interdisziplinäre Projekte stehen. Diese sollten auf signifikante technologische Innovationen abzielen und in einem Zeitrahmen von etwa drei bis acht Jahren neue (wirtschaftliche) Anwendungsfelder erschließen. Als Ergebnis eines FIT-IT Forschungsvorhabens sollte beispielsweise der Funktionsnachweis einer technologischen Lösung, z. B. in Form eines Prototyps stehen. Reine Produktentwicklung wurde mit dem Impulsprogramm FIT-IT nicht gefördert. 


\section{Zielgruppen und Form der Förderung}

Zur Teilnahme und Einreichung von Projektvorschlägen im Impulsprogramm FIT-IT waren Unternehmen der gewerblichen Wirtschaft, insbesondere auch neu gegründete Unternehmen (Spin-offs, Start-ups), wissenschaftliche Einrichtungen und Forschungsinstitute bzw. deren Rechtsträger, Arbeitsgemeinschaften aber auch Einzelforscher/innen berechtigt.

Mit dem FIT-IT Programm wurden primär kooperative Projekte unterstützt. Die Konsortien für die Forschungsprojekte mussten aus mindestens zwei Partnern bestehen. Mindestens ein Konsortialpartner sollte ein Unternehmen der gewerblichen Wirtschaft sein, um den Anwendungskontext des Forschungsvorhabens schon in einem möglichst frühen Stadium sicherzustellen. Die Anzahl der Projektteilnehmer war formal nicht begrenzt. In der Evaluierung der Projektanträge wurde geprüft, ob die Zusammensetzung des Konsortiums zweckmäßig war. Außerdem konnten im Impulsprogramm FIT-IT Begleitmaßnahmen und Ausbildungsprojekte finanziert werden, die auch von einem einzelnen Antragsteller eingereicht werden konnten.

Hinsichtlich Dauer und finanziellem Umfang der Projekte bestanden keine Vorgaben. In der Praxis wurden überwiegend Projekte zur Förderung eingereicht, die etwa auf zwei bis drei Jahre Projektdauer ausgelegt waren. Die Einreichung von Projekten erfolgte auf Grundlage von Ausschreibungen, in denen thematisch inhaltliche Schwerpunkte gesetzt werden konnten. Hinsichtlich der technologischen Problemlösung war der Anspruch des Programms, der kreativen Vielfalt von Forschung Raum zu geben.

\section{Ausschreibungsschwerpunkte und Auswahlverfahren}

Die Festlegung der Ausschreibungsschwerpunkte erfolgte in einem Dialog mit Fachexpert/inn/en, in dessen Rahmen zahlreiche Informationsveranstaltungen über die Inhalte aktueller Ausschreibungen, aber auch über Erfolge bereits geförderter Projekte oder über aktuelle Entwicklungen berichtet wurde. Auch die Erfahrungen des Programmmanagements und Rückmeldungen der Einreicher/innen aus durchgeführten Ausschreibungen wurden für die Weiterentwicklung des Programms berücksichtigt und dienten als Input für zukünftige Ausschreibungen.

Die Evaluierung der eingereichten Anträge wurde durch Panels internationaler Expert/inn/en durchgeführt. Die Auswahl der Expert/inn/en für die Panelevaluierungen erfolgte durch das BMVIT mit Unterstützung des Programmmanagements. Die Auswahljurys erstellten unabhängig von BMVIT und dem Programmmanagement einen Vorschlag für die Reihung / Förderung der eingereichten Anträge. Die Förderentscheidung wurde auf Grundlage der Ergebnisse des Auswahlverfahrens durch den Bundesminister / die Bundesministerin für Verkehr, Innovation und Technologie getroffen.

\section{Programmmanagement}

Eine Besonderheit - zumindest aus heutiger Sicht - in der ersten Phase des FIT-IT Programms war die Betrauung eines privaten Unternehmens mit dem operativen Programmmanagement. Das FIT-IT Programm startete bereits einige Jahre vor Gründung der FFG Österreichische Forschungsförderungsgesellschaft. Eine ihrer Vorgängerorganisationen - der FFF Forschungsförderungsfonds für die Gewerbliche Wirtschaft - nahm zwar von Beginn an die fördertechnische Abwicklung der bewilligten FIT-IT Projekte vor, die Kommunikation und Betreuung der Antragsteller/innen in den Ausschreibungen erfolgte jedoch durch die vom BMVIT beauftragte private Agentur.

Dies war in den späten 1990er Jahren und in frühen $2000 e r$ Jahre eine gängige Konstruktion bei der Abwicklung von thematischen FuE-Programmen des heutigen BMVIT. Erst nach der 
Gründung der FFG, dem damit verbundenen Aufbau von Expertise und zusätzlicher personellen Ressourcen für das Management thematischer Programme wurde auch das Programmmanagement des Impulsprogramms FIT-IT ganz von der FFG übernommen. Seit 2007 war die FFG alleine für die operative Programmdurchführung zuständig.

\section{Programmlinien}

Das Impulsprogramm FIT-IT startete im Jahr 2002 mit den zwei Programmlinien „Embedded Systems" und "Semantic Systems" (Semantische und intelligente Systeme und Dienste). Diese Themen spiegelten zu diesem Zeitpunkt auch europäische Förderschwerpunkte im Rahmenprogramm der Europäischen Union wieder. Das Programm FIT-IT diente damit der Ausrichtung und Abstimmung österreichischer Forschungsschwerpunkte mit jenen der Europäischen Union, um Synergien zwischen nationaler und europäischer Forschung zu sichern und die längerfristigen Erfolgsaussichten österreichischer Einreicher in den entsprechenden Schwerpunkten des EU-Rahmenprogramms zu verbessern.

Bis zum Jahr 2006 wurde das Impulsprogramm FIT-IT um die drei Programmlinien „Systems on Chip", "Trust in IT Systems" und "Visual Computing" erweitert. Daneben wurden im Impulsprogramm FIT-IT immer wieder auch neue Akzente gesetzt bzw. neue Förderschwerpunkte des BMVIT aufgegriffen. Mit der Förderinitiative ModSim Computational Mathematics sollten beispielsweise ab 2007 die in Österreich vorhandenen Kapazitäten auf dem Gebiet der Modellierung und Simulation gestärkt werden. Die Förderinitiative zielte dabei primär auf strukturwirksame Maßnahmen ab, um die vorhandene akademische Expertise für die wirtschaftliche Entwicklung zu nutzen und die Anwendung von Computational Mathematics in der Wirtschaft zu verbreitern. Die Förderung sollte damit die Absorptionskapazität der Unternehmen für Computational Mathematics erhöhen. Gefördert wurden sowohl kooperative Projekte als auch Einzelanträge.

\section{Projektarten}

Für die Förderung von Projekten wurden im Impulsprogramm FIT-IT hauptsächlich drei Projektarten eingesetzt: Die wichtigste Projektart war das "Kooperative Forschungsprojekt", das in erster Linie gemeinsam durchgeführte Projekte der industriellen Forschung zwischen Unternehmen und Forschungseinrichtungen förderte. Die Anforderungen an die Zusammenarbeit der Konsortien und die (Mindest-)Anteile an den Projektkosten für Forschungspartner und Unternehmenspartner wurden über die Laufzeit des Programms mehrfach angepasst. Die Mindestwerte für zugeordnete FuE-Leistungen der Partner sollten den kooperativen Charakter der Projekte sicherstellen. Für die Projektanteile kleiner, mittlerer und großer Unternehmen wurden unterschiedliche Förderquoten gewährt, um die Beteiligung von KMU besonders zu stimulieren. Da die beteiligten Forschungspartner (d. h. Hochschulen und auBeruniversitäre Forschungseinrichtungen) keine vollständige Förderung der an ihren Einrichtungen anfallenden Kosten erhielten, waren diese von den an den Projekten beteiligten Unternehmen auszufinanzieren.

Daneben förderte FIT-IT in kleinerem Umfang Ausbildungsprojekte bzw. vergab Dissertationsstipendien zu Themen in den FIT-IT Programmlinien. Dissertationsstipendien wurden vom Institut eingereicht, an dem die Dissertation durchgeführt werden sollte. Die Höhe des Stipendiums richtete sich danach, ob die Promovierenden nur am Institut oder auch in einem Unternehmen beschäftigt waren. Waren die Promovierenden Teilzeit in einem Unternehmen angestellt, so konnten die anfallenden Personalkosten für die restliche Zeit am Institut auf Basis der Höhe des Firmengehalts gefördert werden. Waren die Promovierenden ausschließlich am Institut beschäftigt, wurden die Personalkosten in der Höhe des entsprechenden Sat- 
zes für Doktorandinnen und Doktoranden beim FWF Fonds zur Förderung der wissenschaftlichen Forschung gefördert. Weitere, im Programm FIT-IT förderbare Ausbildungsmaßnahmen waren beispielsweise Summer Schools oder der Personalaufbau bei neuen, thematisch fokussierten Forschungsteams. Schließlich finanzierte das Impulsprogramm FIT-IT über die Jahre auch zahlreiche begleitende Maßnahmen wie Studien, Maßnahmen der Öffentlichkeitsarbeit oder wissenschaftliche Konferenzen.

Im Jahr 2007 wurde im Impulsprogramm FIT-IT außerdem die BMVIT-Konzeptinitiative „Forschung schafft Arbeit" aufgegriffen. Das BMVIT förderte damit die Aktivitäten von Kleinstunternehmen bis zu neun Beschäftigten (einschließlich Einzelunternehmen, Einzelpersonen, neue Selbstständige), um neue und innovative Ideen mit technologischem und wirtschaftlichem Verwertungspotenzial aus diesem Adressatenkreis frühzeitig aufzugreifen. Dadurch sollte die wissenschaftliche und industrielle Basis für Innovationen verbreitert, Stärken aufgebaut und neue Märkte erschlossen werden. Die Förderung konnte zur Vorbereitung auf nationale oder internationale Ausschreibungen gewährt werden oder auch als Vorstufe zu oder als begleitende Unterstützung bei der Unternehmensgründung dienen. Die Anträge in der Konzeptinitiativen mussten nicht den FIT-IT Programmzielen entsprechen, jedoch thematisch auf Informationstechnologie ausgerichtet sein. Gefördert wurden Projekte bis zu 35 Tsd. Euro und einer Laufzeit von maximal zwölf Monaten mit einer Förderquote von 100 \%.

\section{Förderthema Demographischer Wandel}

Daneben entwickelte sich das BMVIT-Programmportfolio im Bereich der IKT-Forschung auch aufgrund neuer (europäischer) Impulse weiter. So wurde beispielsweise Mitte der 2000er Jahre das Thema IKT und demographischer Wandel bzw. IKT und die alternde Gesellschaft als wichtiges Handlungsfeld identifiziert. Das BMVIT war in der Folge sehr aktiv bei der Vorbereitung der Einrichtung des transnational organisierten Ambient Assisted Living (AAL) Joint Programms (2008 - 2013), das seit 2014 als „Active and Assisted Living Programme" weitergeführt wird. Als nationaler Programmbeitrag bzw. als nationale Schnittstelle zur transnationalen Zusammenarbeit im AAL-Programm wurde dazu das Programm benefit eingerichtet.

Seit dem Jahr 2007 fördert das BMVIT mit dem Programm benefit IKT-Themen, die in Zusammenhang mit der Bewältigung des demographischen Wandels im Sinne der alternden Gesellschaft stehen. Die Finanzierung des transnationalen AAL-Programms erfolgt ebenfalls aus nationalen Mitteln der beteiligten Länder sowie aus Beiträgen der Europäischen Kommission auf Grundlage des Artikels 185 des Vertrags über die Arbeitsweise der Europäischen Union (AEUV), mit dem die Teilnahme der Europäischen Union an der gemeinsamen Durchführung von FuE-Programmen der Mitgliedsstaaten ermöglicht wird.

Im Zentrum der Programme benefit und AAL stehen Forschung, Technologieentwicklung und Innovation zur Bewältigung von gesellschaftlichen Herausforderungen im Zusammenhang mit der Alterung der Gesellschaft. Durch den effektiven Einsatz von IKT-Lösungen sollen soziale Innovationen ermöglicht und gleichzeitig die Wettbewerbsfähigkeit Österreichs gesteigert werden. Durch IKT-unterstützte Produkte und Dienstleistungen soll Menschen möglichst lange ein unabhängiges Leben in den eigenen vier Wänden ermöglicht werden. Die aus Mitteln des Programms entwickelten Technologien sollen dazu beitragen, die Bedürfnisse und das individuelle Wohlbefinden der Nutzer/innen von AAL-Lösungen zu steigern oder durch IKT-unterstützte Ansätze zur Prävention von chronischen Krankheiten beitragen. Neue nutzerorientierte Geschäftsmodellen und innovative Wertschöpfungsketten stehen ebenfalls besonders im Zentrum der Förderung. Damit will das Programm benefit 
frühzeitig Nischen für die heimische Wirtschaft schaffen und deren internationale Vermarktung fördern.

Der transnationale Teil der FuE-Förderung zur Bewältigung des demographischen Wandels (AAL) wird von der FFG gemeinsam mit der von den nationalen Ministerien und Agenturen eingerichteten Trägerorganisation des Forschungsprogramms, der AAL Association (AALA) mit Sitz in Brüssel, abgewickelt. Die Förderung der Projektpartner erfolgt entlang der Bedingungen und Bestimmungen in den jeweiligen nationalen FuE-Programmen. Das BMVIT beteiligt sich mit Fördermitteln an den jährlichen Ausschreibungen, wobei bei Bedarf auch Mittel aus dem Programm benefit genutzt werden können. AAL fördert FuE-Projekte, an denen mindestens drei Projektpartner aus verschiedenen Partnerländern beteiligt sind, darunter zwingend ein Partner aus dem Unternehmensbereich. Die FFG prüft bei Einreichungen die Einhaltung der Beteiligungskriterien. Die Evaluation der Projektanträge erfolgt zentral durch eine internationale Jury. Da mit den gewidmeten nationalen Mitteln nur Partner aus den jeweiligen Ländern gefördert werden, kann es im Zuge der Selektionsphase zu Anpassungen in der Partnerstruktur der Projektanträge kommen, um die zur Verfügung stehenden Mittel einschließlich der EU-Beiträge - bestmöglich ausschöpfen zu können.

\section{Gemeinsame Technologieinitiativen ARTEMIS und ENIAC}

Im Bereich Mikro- und Nanoelektronik und im Bereich Embedded Systems hatten sich die bereits Anfang der 2000er Jahre industriegetriebene Technologieplattformen etabliert, die europäische Forschungsagenden ausgearbeitet hatten. Zur Umsetzung dieser Forschungsagenden wurden schließlich von der Europäischen Union Gemeinsame Technologieinitiativen (GTI) beschlossen, die über eigens eingerichtete institutionelle Vehikel in Form von öffentlich-privaten Partnerschaften (den GU Gemeinsamen Unternehmen) unter Beteiligung der Industrie, der Europäischen Union und der Mitgliedsländern realisiert wurden.

Um sich effektiv an den unter ARTEMIS (Embedded Systems) und ENIAC (Nanoelektronik) bezeichneten Programmen beteiligen zu können, brauchte es entsprechende nationale strategische, organisatorische und finanzielle Kapazitäten, die durch das BMVIT mittels direkter (Förderung) und indirekter Maßnahmen (Unterstützung des Aufbaus nationaler Plattformen) auch aufgebaut wurden. Ab dem Jahr 2007 wurden die beiden Gemeinsamen Technologieinitiativen ARTEMIS und ENIAC schließlich unter aktiver Beteiligung des BMVIT umgesetzt.

\section{Neukonzeption der BMVIT IKT-Forschungsprogramme}

Durch die Aktivitäten und Maßnahmen des BMVIT war über die Jahre das thematische Portfolio des Impulsprogramms FIT-IT stark angewachsen. Auch das Programmumfeld hatte sich sehr deutlich gegenüber dem Start des Impulsprogramms FIT-IT gewandelt. Innerhalb des BMVIT setzte daher zu Beginn der 2010er Jahre ein Prozess ein, mit dem die unterschiedlichen Programme und Maßnahmen unter dem Begriff Themenmanagement stärker aufeinander abgestimmt und konsolidiert werden sollten. Für das Impulsprogramm FIT-IT war das BMVIT auch zur Überzeugung gekommen, dass einzelne Programmziele bzw. Programmansprüche (wie beispielsweise die Abstimmung mit europäischen Förderthemen oder die Heranführung österreichischer Akteure an europäische Programme) bereits erreicht worden waren und daher in dieser Form förderpolitisch nicht mehr als prioritär galten.

Im Jahr 2011 startete unter der Bezeichnung Themeninitiative "IKT der Zukunft" die Neukonzeption und Neugestaltung des Programms unter Einbeziehung von zahlreichen Expert/inn/en. Unter dem neuen Programmschirm wurde die nationale IKT-Förderung stärker anwendungsfeldbezogen ausgerichtet und mit der Förderung der österreichischen Beteiligung an den Gemeinsamen Technologieinitiativen ARTEMIS und ENIAC sowie seit 2014 auch 
mit den nationalen und transnationalen Programmen für die Bewältigung des demographischen Wandels (AAL, benefit) zusammengefasst.

Die Themeninitiative IKT der Zukunft verfolgt nunmehr die folgenden Ziele:

- Spitzentechnologien weiterentwickeln

- Spitzenpositionen im Wettbewerb erzielen

- Spitzenpositionen als Forschungsstandort ausbauen bzw. neu einnehmen

- Spitzenkräfte bereitstellen und gewinnen

Bereits die letzte FIT-IT Ausschreibung des Jahres 2011 stellte einen Übergang zwischen dem Impulsprogramm FIT-IT und der Themeninitiative "IKT der Zukunft" dar. Die FIT-IT Ausschreibung 2011 war die erste, in der die Förderschwerpunkte Embedded Systems, Semantic Systems, Trust in IT Systems und Visual Computing zusammen ausgeschrieben wurden. Gleichzeitig wurden die Ausschreibung unter das gemeinsame Thema „Komplexe IKT-gestützte Systeme - Entwicklung und Sicherheit" gestellt.

\section{Fokus auf Förderthemen und Anwendungsfelder}

Ab 2012 wurden die Ausschreibungen schließlich unter der neuen Programmbezeichnung „IKT der Zukunft" durchgeführt. IKT der Zukunft konzentriert sich inhaltlich nunmehr auf vier Förderthemen, die in jeweils drei Subthemen unterteilt sind:

- Komplexe IKT-Lösungen ermöglichen (Systems of Systems) mit den Subthemen a) Rigorose Entwurfsmethoden, b) Adaptivität und Weiterentwicklung und c) Autonomie;

- Vertrauen rechtfertigen (Sichere Systeme) mit den Subthemen a) Safety \& Security by Design, b) Usable Security und c) Ubiquitous Security;

- Daten durchdringen (Intelligente Systeme) mit den Subthemen a) Suche und Analyse, b) Semantische Verarbeitung und c) Kognitive Systeme;

- Interoperabilität gewährleisten (Schnittstellen von Systemen) mit den Subthemen a) Schnittstellenkonzepte, b) Kompatibilität und c) Technologien und Werkzeuge für Schnittstellen.

Dazu kommen mit a) Human-Centered Computing, b) Schonender Umgang mit Ressourcen und c) Bewusster Umgang mit F\&E-Daten drei inhaltliche Querschnittsthemen.

Diese Themen werden in den IKT der Zukunft-Ausschreibungen an verschiedenen Anwendungsfelder wie Produktion, Energiesysteme, Telekommunikation, Weltraum oder gesellschaftlichen Fragen wie dem demographischen Wandel gespiegelt. Mit dem Programm sollen IKT-spezifische Herausforderungen adressiert werden, für deren Lösung Grundfragen der Informatik, Elektronik, Software- oder Hardwareentwicklung notwendig sind, um in den jeweiligen Anwendungsfeldern Innovationen auslösen zu können.

Förderanträge, die nicht in einem engen Zusammenhang mit einem der zwölf Themen stehen, werden im Unterschied zur früheren, im Impulsprogramm FIT-IT gepflegten Praxis, nur mehr dann gefördert, wenn dies in den Ausschreibungen explizit vorgesehen ist bzw. Budget für Themen außerhalb der definierten Anwendungsfelder reserviert wurde.

Die dominierende Projektart blieb weiterhin das kooperative FuE-Projekt (für industrielle Forschung oder experimentelle Entwicklung). Daneben können Sondierungsprojekte und FuEDienstleistungsprojekte zu definierten Fragestellungen gefördert werden, die z. B. der Vorbereitung und Abstimmung mit europäischen Forschungsschwerpunkten dienen können. 


\section{Einführung neuer, strukturell wirksamer Förderinstrumente}

Das BMVIT als Programmeigentümer versucht mit der Themeninitiative IKT der Zukunft immer wieder auch besonders strukturell wirksame Maßnahmen zu setzen, das heißt solche, deren Ziele mit klassischen kooperativen FuE-Projekten als Projektart nicht erreicht werden können. Darunter fallen beispielsweise Leitprojekte oder Stiftungsprofessuren. Auch mit der Ausschreibung von Modellregionen im Programm benefit verfolgte das BMVIT einen Förderansatz, mit dem der beschränkte Wirkrahmen der kooperativen FuE-Projektförderung überwunden werden sollte.

\section{Diffusion von IKT als Forschungsthema in andere thematische Programme}

Für diese Evaluierung relevant ist auch die Tatsache, dass sich seit Start des Impulsprogramms FIT-IT das Programmumfeld stark weiterentwickelt hat. Im Jahr 2002 war FIT-IT das erste unmittelbar auf IKT-Forschung abzielende thematische bzW. missionsorientierte FuEProgramm des BMVIT. In den folgenden Jahren wurden vom BMVIT selbst, aber auch von den für Wissenschaft und Wirtschaft zuständigen Ministerien, zahlreiche andere Initiativen und Programme beauftragt, die (auch) IKT-Themen aufgreifen. Dies geschah überwiegend durch eine zunehmende Orientierung auf wirtschaftliche und gesellschaftliche Herausforderungen, in deren Dienst neue Forschungs- und Innovationsprogramme gestellt wurden. In den letzten Jahren war es vor allem das Thema Digitalisierung, das aufgrund seiner praktisch allgegenwärtigen und horizontalen Relevanz für Innovationen in Wirtschaft und Gesellschaft auch die IKT-Forschung bzw. die IKT-Forschungsförderung massiv beeinflusste.

Gemessen an den verfügbaren Budgets für thematische FuE-Programme ist daher die Bedeutung des nationalen BMVIT IKT-Programms über die Jahre zurückgegangen. In der FFGFörderstatistik des Jahres 2006 machten die FIT-IT Förderungen noch 19,3\% der insgesamt für thematische Programme der FFG gewidmeten Förderungen aus. Im Förderjahr 2016 waren es hingegen nur mehr 12,2 \% (ohne benefit). Gleichzeitig gibt es deutlich mehr Förderangebot mit substanziellen IKT-Forschungsanteilen in anderen thematischen Programmen, wie Produktion der Zukunft, Mobilität der Zukunft, Energie der Zukunft oder Smart Cities.

IKT als Forschungsthema hat mit diesen Entwicklungen jedoch sicher nicht an Bedeutung verloren - ganz im Gegenteil. Allerdings hat sich der Fokus der Forschungsförderung insgesamt von einem eher wissenschaftlich-technisch orientierten Ansatz hin zu stärker anwendungs- und problemorientierten Fragestellungen verschoben. Die ist eine Entwicklung, die nicht nur in Österreich zu beobachten war, sondern auch sehr deutlich in anderen europäischen Ländern (siehe dazu Anhang 2).

\subsection{Ergebnisse bereits vorliegender Evaluierungen}

Einzelne Teile des Impulsprogramms FIT-IT bzw. der Themeninitiative "IKT der Zukunft" waren bereits in der Vergangenheit Gegenstand von Evaluierungen, deren Ergebnisse wir an dieser Stelle kurz zusammenfassen und sie später in der Diskussion unserer eigenen Befunde und Schlussfolgerungen zu berücksichtigen.

\section{FIT-IT Zwischenevaluierung 2005}

Für das Impulsprogramm FIT-IT wurde im Jahr 2005 eine erste Zwischenevaluierung durchgeführt (Zinöcker et al. 2005). Das Evaluatorenteam kommt dabei zum Schluss, dass mit dem Programm ein sinnvoller Schwerpunkt gesetzt wurde und das Design des Programms zweckmäßig ist. Auch wurde in der Befragung der Antragsteller/innen dem vom BMVIT beauftragten Programmmanagement für die Abwicklung ein sehr gutes Zeugnis ausgestellt. 
Über die Additionalität der Förderung konnten in der Zwischenevaluierung noch keine belastbaren Aussagen gemacht werden. Erste Hinweise hätten jedoch auf positive Effekte hingedeutet. Gleichzeitig wurde auf Mitnahmeeffekte hingewiesen, da überwiegend Konsortien gefördert wurden, die bereits zuvor zusammengearbeitet hatten.

Die Evaluator/inn/en empfahlen eine Konzentration des Programms auf kooperative Forschungsprojekte und rieten vor allem von einer Ausweitung der eingesetzten Förderinstrumente ab, um andere Programme und Maßnahmen nicht zu duplizieren.

\section{Evaluierung der österreichischen Beteiligung an AAL 2015}

Die österreichische Beteiligung am Ambient Assisted Living Joint Programme (AAL JP 2008 - 2013) wurde im Jahr 2015 evaluiert (Geyer und Good 2016). Um einen Vergleich mit der nationalen Förderung zu ermöglichen, wurden in dieser Evaluierung auch Projekte des Programms benefit mitberücksichtigt.

Die Evaluierung zeigte, dass die nationale Förderschiene benefit thematisch deutlich mehr Diversität ermöglicht und auch stärker experimentell ausgerichtete Projekte zulässt, als das transnationale AAL-Programm. Größter Mehrwert des AAL-Programms ist die erfolgreiche Einbeziehung von nicht-klassischen Projektpartnern in FuE-Vorhaben, wie Daseinsvorsorgern, Hilfsorganisationen und Nutzer/innen von AAL-Anwendungen. Dieser Umstand wird auch von den beteiligten Forschungseinrichtungen und Hochschulen als äußerst hilfreich und befruchtend bewertet.

Gleichzeitig zeigte die Evaluation, dass die wirtschaftlichen Programmziele (noch) nicht erreicht werden konnten bzw. sich als zu ambitioniert herausgestellt haben. Der im Programm angestrebte Verwertungshorizont von Projektergebnissen von zwei bis drei Jahren nach Projektende konnte in den meisten Fällen nicht erreicht werden. Die Evaluator/inn/en schlugen vor, zukünftig noch stärker auf die Einbeziehung von Schlüsselakteuren aus dem Gesundheits-, Sozial- und Pflegewesen zu achten, um die Praxisrelevanz der AAL-Lösungen möglichst gut zu gewährleisten.

\section{Europäische AAL Evaluierungen}

In der letzten Evaluierung des Active \& Assisted Living Programme (Uusikylä et al. 2017) kommt die Expert/inn/engruppe zum Schluss, dass der Übergang vom ersten Ambient Assisted Living Joint Programme (AAL JP 2008 - 2013) auf das aktuelle Programm mit Blick auf die angestrebten Ziele insgesamt gut gelungen sei. Das Programm habe nach wie vor ein klares Alleinstellungsmerkmal in europäischen Kontext, sei thematisch weiterhin hoch relevant und liefere daher auch einen klaren Mehrwert für Europa.

Sehr kritisch merkte die Expert/inn/engruppe allerdings an, dass durch die im Vergleich zum ersten AAL-Programm deutlich geringere (finanzielle) Beteiligung der Mitgliedsstaaten die Nachhaltigkeit einer kritischen Masse an FuE- und Innovationsaktivitäten auf europäischer Ebene nicht mehr gesichert sei. Das Programm brauche mehr strategische Führung, was vor allem die beteiligten Mitgliedsstaaten als „Eigentümer" des Programms in die Pflicht nehme.

Bezüglich der Verwertung von Projektergebnissen konstatierte die Expert/inn/engruppe, dass es trotz der gesetzten Maßnahmen, um die Marktrelevanz und die Weiterentwicklung von Ergebnissen in Produkte und Dienstleistungen zu steigern, nach wie vor an marktfähigen Ergebnissen mangle. Das Programm sollte hier noch stärker auf Entwicklungen am Markt reagieren. Insbesondere sei der Fokus auf KMU und Endnutzer/inn/en (d. h. ältere Personen) alleine nicht geeignet, erfolgreich Produktentwicklung zu machen. Große Unternehmen, aber 
auch sekundäre und tertiäre Nutzer (einschließlich Gebietskörperschaften) sollten intensiver in die Projekte integriert werden.

\section{Zwischenevaluierung ARTEMIS und ENIAC 2011}

In einer im Jahr 2011 durchgeführten Zwischenevaluierung (Geyer 2012) wird ein durchwachsenes Bild des Erfolgs der österreichischen Beteiligung an ARTEMIS und ENIAC zum damaligen Zeitpunkt gezeichnet. Wird als Beurteilungsmaßstab der Umfang der Projektbeteiligungen bzw. der österreichische Anteil am Gesamtprojektumfang herangezogen, so war die Bilanz in beiden Initiativen sehr positiv. Mit 7,1\% des Gesamtprojektvolumens in ARTEMIS bzw. $5,2 \%$ in ENIAC lag der österreichische Anteil deutlich über dem damaligen BIP-Anteil Österreichs innerhalb der Europäischen Union oder auch über den IKT-Vergleichszahlen im 7. EUForschungsrahmenprogramm (ca. 4,0\%). Der österreichische Projektanteil an ARTEMIS und ENIAC alleine würde jedoch keinen aussagekräftigen Erfolgsindikator darstellen, da die verfügbaren nationalen Finanzierungsbudgets in einem hohen Ausmaß die Beteiligungsmöglichkeiten von Partnern in den verschiedenen Ländern an den Projekten bestimmen würden.

Auf der positiven Seite seien aber jedenfalls die Netzwerkeffekte zu nennen, die von vielen österreichischen Projektpartnern festgestellt wurden. Auch die bessere Vernetzung und Abstimmung der österreichischen Forschungsakteure untereinander und mit den zuständigen Stellen im BMVIT durch Einrichtung von nationalen Plattformen wurde als Erfolg gewertet. Eines der Ziele des BMVIT mit den Initiativen ARTEMIS und ENIAC sei es aber auch gewesen, einer größeren Anzahl an österreichischen Akteuren Zugang zu den wesentlichen europäischen Konsortien und Netzwerken zu verschaffen. Dieses Ziel sei nur sehr eingeschränkt erreicht worden. Die Liste der erfolgreichen Organisationen aus Wirtschaft und Wissenschaft habe nur die "usual suspects" umfasst.

Als besonders auffällig wurde bemerkt, dass sich bis zum Zeitpunkt der Zwischenevaluierung mehr österreichische Einrichtungen an ENIAC beteiligen konnten als an ARTEMIS, obwohl die Stakeholder ursprünglich das Beteiligungspotenzial Österreichs an ARTEMIS deutlich höher eingeschätzt hatten als bei ENIAC. Fördertechnisch wurde vorgeschlagen, die effektiven Förderintensitäten (d. h. Summe von EU-Beitragsanteil und nationaler Förderung) bei ARTEMIS und ENIAC-Projekten jenen von EUREKA-Cluster-Projekten anzupassen.

\section{Europäische Evaluierungen der Gemeinsamen Technologieinitiativen}

Die Gemeinsamen Technologieinitiativen ARTEMIS und ENIAC, die seit 2014 in gemeinsamer Form unter ECSEL fortgesetzt wurden, waren wiederholt Gegenstand von Zwischen- und Endevaluierungen der Europäischen Kommission. Dazu kamen zahlreiche weitere Untersuchungen zur Prüfung der Verfahren und Abläufe, insbesondere hinsichtlich der finanziellen Gebarung der für die Umsetzung der Initiativen eingerichteten Gemeinsamen Unternehmen (ARTEMIS, ENIAC bzw. ECSEL Joint Undertaking).

Die finanzielle Gebarung der Gemeinsamen Unternehmen war in der Vergangenheit immer wieder Gegenstand der Kritik des Europäische Rechnungshofs und des Europäischen Parlaments. Die Kritik betraf beispielsweise die bestehenden Kontrollmechanismen, um die Einhaltung der anzuwendenden Europäischen Finanzregeln sicherzustellen. Der Budgetkontrollausschuss des Europäischen Parlaments verweigerte den beiden Gemeinsamen Unternehmen ARTEMIS und ENIAC für die Jahre 2013 und 2014 vorerst auch die Entlastung, weil sie die Abgeordneten nicht von der ordnungsgemäßen Verwendung der eingebrachten EUMittel überzeugen konnten (EP 2015, EP 2016). Erst nach einer Reihe von Maßnahmen zur 
Erhöhung der Transparenz der Mittelvergabe und der Etablierung eines Ex-Post Auditmechanismus zur Prüfung der vertraglichen Vereinbarungen mit den beteiligten nationalen Förderstellen wurde eine Entlastung erteilt.

In der aktuellsten inhaltlichen Evaluierung (Thompson et al. 2017) kommt die von der Europäischen Kommission eingesetzte Expert/inn/engruppe zum Schluss, dass ECSEL und die beiden Vorläuferinitiativen sehr erfolgreich waren, die Interessen der europäischen Mikround Nanoelektronikindustrie zu unterstützen. Mit den Programmen wurden Entwicklungen gefördert, um die Produktionsmöglichkeiten für modernste siliziumbasierte Fertigungsverfahren und das dazu notwendige Know-how in Europa zu halten. Gleichzeitig sahen die Expert/inn/en einen weiterhin großen Bedarf nach besserer vertikaler Integration der entwickelten Technologie. Zudem sei eine noch bessere Abstimmung der nationalen Aktivitäten, Förderbedingungen und Förderverfahren notwendig. Außerdem sollten Maßnahmen getroffen werden, um das Beteiligungsziel für KMU in Horizon 2020 im Umfang von 20 \% der bewilligten Mittel zu erreichen.

Schließlich wurde noch darauf hingewiesen, dass es geeignete Indikatoren und ein verpflichtendes Wirkungsmonitoring hinsichtlich der erzielten Ergebnisse der geförderten Projekte braucht, um das finanzielle Engagement der Kommission, der Mitgliedsstaaten und der Industrie rechtfertigen zu können. 
In welchem Umfang wurden die Ausschreibungen in den BMVIT IKT-Programmen von den Zielgruppen angenommen? Wer hat sich an den Ausschreibungen beteiligt und mit welchem Erfolg? Wie haben sich die Beteiligungszahlen, die Bewilligungen und die Budgets in den BMVIT IKT-Programmen seit 2002 entwickelt? Haben sich die Schwerpunkte der Förderung nach dem Übergang vom Impulsprogramm FIT-IT zur Themeninitiative IKT der Zukunft verändert? Diese Fragen stehen im Zentrum dieses Abschnitts.

\subsection{Beteiligung nach Programmlinien und Förderthemen}

Die folgenden Tabellen geben einen Überblick zu den Beteiligungskennzahlen im FIT-IT Programm in den Jahren 2002 bis $2012{ }^{1}$

\section{Eingereichte und bewilligte Projekte}

Insgesamt wurden in den einzelnen Programmen und Programmlinien über die Laufzeit des Impulsprogramms FIT-IT mehr als 1.000 Projekte (kooperative FuE-Projekte, Sondierungsprojekte, Begleitprojekte und Beauftragungen) eingereicht. Rund $67 \%$ der Projektanträge entfielen dabei auf die Programmlinien des Impulsprogramms FIT-IT. Etwa $9 \%$ der Anträge wurden für österreichische Beteiligungen an den Gemeinsamen Technologieinitiativen ARTEMIS und ENIAC eingereicht. Rund 24 \% der Anträge kamen für Projekte zum Demographischen Wandel.

Tabelle 1 Impulsprogramm FIT-IT: Anzahl der eingereichten und bewilligten Projektanträge nach Programmen / Programmlinien 2002-2012

\begin{tabular}{|c|c|c|c|}
\hline Programm / Programmlinie & $\begin{array}{c}\text { Projektanträge } \\
\text { eingereicht }\end{array}$ & $\begin{array}{c}\text { Projektanträge } \\
\text { genehmigt }\end{array}$ & $\begin{array}{c}\text { Anteil } \\
\text { genehmigt (\%) }\end{array}$ \\
\hline Impulsprogramm FIT-IT & $\mathbf{6 7 4}$ & $\mathbf{3 1 7}$ & $\mathbf{4 7 \%}$ \\
\hline Embedded Systems & 198 & 103 & $52 \%$ \\
\hline Semantic Systems & 152 & 64 & $42 \%$ \\
\hline Systems on Chip & 35 & 18 & $51 \%$ \\
\hline Trust in ITS & 59 & 24 & $41 \%$ \\
\hline Visual Computing & 99 & 50 & $51 \%$ \\
\hline Initiativen \& Joint Call & 131 & 58 & $44 \%$ \\
\hline Gemeinsame Technologieinitiativen & $\mathbf{9 1}$ & $\mathbf{4 6}$ & $\mathbf{5 1 \%}$ \\
\hline ARTEMIS & 48 & 19 & $40 \%$ \\
\hline ENIAC & 43 & 27 & $63 \%$ \\
\hline Demographischer Wandel & $\mathbf{2 4 6}$ & $\mathbf{1 1 0}$ & $\mathbf{4 5 \%}$ \\
\hline AAL & 126 & 46 & $37 \%$ \\
\hline benefit & 120 & 64 & $53 \%$ \\
\hline Gesamtzahl Projekte & $\mathbf{1 . 0 1 1}$ & $\mathbf{4 7 3}$ & $\mathbf{4 7 \%}$ \\
\hline
\end{tabular}

Quelle: FFG, eigene Berechnung inspire research

Die Bewilligungsquote der Projektanträge lag bei durchschnittlich $47 \%$. Deutlich niedrigere Bewilligungsquoten gab es bei den transnationalen AAL-Projekten zum Demographischen

\footnotetext{
${ }^{1}$ Mit „FIT-IT Programm" bezeichnen wir in der Folge die nationale FIT-IT Förderung einschließlich der während der Programmlaufzeit durchgeführten ARTEMIS / ENIAC- sowie AAL / benefit-Ausschreibungen). MIT „FIT-IT Programmlinien" bezeichnen wir ausschließlich die nationale FIT-IT Förderung. Mit "Gemeinsame Technologieinitiativen“ bezeichnen wir die Programme ARTEMIS und ENIAC (bzw. ECSEL). Mit „Demographischer Wandel“ bezeichnen wir die Programme benefit und AAL.
} 
Wandel (37\%), bei Anträgen zur Gemeinsamen Technologieinitiative ARTEMIS (40 \%) und bei den Anträgen in der FIT-IT Programmlinie Trust in IT-Systems (41\%).

Überdurchschnittlich hohe Projektbewilligungsquoten lassen sich für die Gemeinsamen Technologieinitiative ENIAC (63\%), die nationalen Projekte zum Demographischen Wandel (53\%) sowie für die Programmlinie Embedded Systems (52 \%) feststellen. Insgesamt deuten die Bewilligungsquoten in den Jahren 2002 bis 2012 auf ein kompetitives Verfahren hin, das jedoch nach wie vor den Projekteinreichern insgesamt angemessene Erfolgschancen für eine Förderung ihrer Anträge in Aussicht stellte.

Ein Vergleich mit den Antragszahlen seit dem Start der Themeninitiative "IKT der Zukunft" zeigt, dass die Bewilligungsquoten der Projektanträge im Vergleich zum Impulsprogramm FIT-IT doch deutlich zurückgegangen sind. Dieser Rückgang betrifft insbesondere die nationale kooperative Projektförderung in der Themeninitiative "IKT der Zukunft" und die Förderung von Projektanträgen zum Demographischen Wandel (AAL und benefit).

Tabelle 2 Themeninitiative „IKT der Zukunft“: Anzahl der eingereichten und bewilligten Projektanträge nach Förderthemen 2012-2016*

\begin{tabular}{|c|c|c|c|}
\hline Förderthemen & $\begin{array}{c}\text { Projektanträge } \\
\text { eingereicht }\end{array}$ & $\begin{array}{c}\text { Projektanträge } \\
\text { genehmigt }\end{array}$ & $\begin{array}{c}\text { Anteil } \\
\text { genehmigt (\%) }\end{array}$ \\
\hline IKT der Zukunft & $\mathbf{3 0 0}$ & $\mathbf{1 0 5}$ & $\mathbf{3 5 \%}$ \\
\hline IKT der Zukunft & 281 & 98 & $35 \%$ \\
\hline IKT der Zukunft Dienstleistungen & 16 & 6 & $38 \%$ \\
\hline IKT der Zukunft Professur & 3 & 1 & $33 \%$ \\
\hline Gemeinsame Technologieinitiativen & $\mathbf{1 6 4}$ & $\mathbf{7 4}$ & $\mathbf{4 5 \%}$ \\
\hline ARTEMIS & 12 & 7 & $58 \%$ \\
\hline ENIAC & 125 & 52 & $42 \%$ \\
\hline ECSEL & 27 & 15 & $56 \%$ \\
\hline Demographischer Wandel & $\mathbf{4 1 6}$ & $\mathbf{1 2 1}$ & $\mathbf{2 9 \%}$ \\
\hline AAL & 285 & 73 & $26 \%$ \\
\hline benefit & 125 & 47 & $38 \%$ \\
\hline benefit Dienstleistungen & 6 & 1 & $17 \%$ \\
\hline Gesamtzahl Projekte & $\mathbf{8 8 0}$ & $\mathbf{3 0 0}$ & $\mathbf{3 4 \%}$ \\
\hline
\end{tabular}

" bis zum 01.08.2017 entschiedene Projekte; Quelle: FFG, eigene Berechnung inspire research

In der nationalen FuE-Projektförderung sank die Bewilligungsquote der Projektanträge von $47 \%$ auf $35 \%$, bei den Anträgen zu nationalen benefit-Projekten von $53 \%$ auf $38 \%$. Noch stärker war der Rückgang bei den transnationalen AAL-Projekten. Hier verringerte sich die durchschnittliche Bewilligungsquote von $37 \%$ auf $26 \%$, wobei hierfür weniger nationale Faktoren ausschlaggebend waren, als vielmehr der Rückgang an verfügbaren Budgets und Kooperationsmöglichkeiten in anderen am AAL-Programm beteiligten Staaten.

\section{Anzahl der an den Projekten beteiligten Partner}

Während der Laufzeit von FIT-IT beteiligten sich mehr als 2.500 Partner an den Projektanträgen. Alleine an den Anträgen für eine nationale Förderung im Impulsprogramm FIT-IT waren mehr als 1750 Partner beteiligt. An den Anträgen der zeitlich später gestarteten Gemeinsamen Technologieinitiativen waren mehr als 200 Partner beteiligt, an Anträgen für Projekte zum Demographischen Wandel mehr als 550 Partner.

Bezogen auf die an den Projektanträgen beteiligten Projektpartner lag die Bewilligungsquote in den Jahren 2002 bis 2012 ähnlich wie unter den Projektanträgen bei knapp unter der Hälfte der insgesamt einreichenden Partner. 
Tabelle 3 Impulsprogramm FIT-IT: Anzahl der Projektpartner in den eingereichten und bewilligten Anträgen nach Programmen / Programmlinien 2002-2012

\begin{tabular}{|c|c|c|c|}
\hline Programm / Programmlinie & $\begin{array}{c}\text { Anzahl Partner } \\
\text { eingereichte Anträge }\end{array}$ & $\begin{array}{c}\text { Anzahl Partner } \\
\text { genehmigte Anträge }\end{array}$ & $\begin{array}{c}\text { Anteil Partner } \\
\text { genehmigt (\%) }\end{array}$ \\
\hline Impulsprogramm FIT-IT & $\mathbf{1 . 7 5 1}$ & $\mathbf{7 8 9}$ & $\mathbf{4 5 \%}$ \\
\hline Embedded Systems & 478 & 226 & $47 \%$ \\
\hline Semantic Systems & 467 & 202 & $43 \%$ \\
\hline Systems on Chip & 92 & 54 & $59 \%$ \\
\hline Trust in ITS & 161 & 61 & $38 \%$ \\
\hline Visual Computing & 257 & 117 & $46 \%$ \\
\hline Initiativen \& Joint Call & 296 & 129 & $44 \%$ \\
\hline Gemeinsame Technologieinitiativen & $\mathbf{2 0 7}$ & $\mathbf{1 0 1}$ & $\mathbf{4 9 \%}$ \\
\hline ARTEMIS & 110 & 51 & $46 \%$ \\
\hline ENIAC & 97 & 50 & $52 \%$ \\
\hline Demographischer Wandel & $\mathbf{5 5 6}$ & $\mathbf{2 7 2}$ & $\mathbf{4 9 \%}$ \\
\hline AAL & 211 & 74 & $35 \%$ \\
\hline benefit & 345 & 198 & $57 \%$ \\
\hline Gesamt Partner & $\mathbf{2 . 5 1 4}$ & $\mathbf{1 . 1 6 2}$ & $\mathbf{4 6 \%}$ \\
\hline
\end{tabular}

Quelle: FFG, eigene Berechnung inspire research

Auch auf Ebene der beteiligten Projektpartner zeigt die Auswertung einen deutlichen Rückgang der Bewilligungsquoten. Erhielten im Impulsprogramm FIT-IT noch $45 \%$ der einreichenden Projektpartner eine Förderung für ihr beantragtes FuE-Vorhaben, ging der entsprechende Anteil in nationalen Projekten von "IKT der Zukunft" auf 35 \% zurück. Eine noch deutliche Tendenz zeigt sich für die Partner in den Projekten zum Demographischen Wandel, während in Projekten der Gemeinsamen Technologieinitiative der Anteil der geförderten Partner unter den Einreichern vergleichsweise stabil geblieben ist.

Tabelle 4 Themeninitiative „IKT der Zukunft": Anzahl der Partner in eingereichten und bewilligten Projektanträgen nach Förderthemen 2012-2016*

\begin{tabular}{|c|c|c|c|}
\hline Themeninitiative „IKT der Zukunft“ & $\begin{array}{c}\text { Anzahl Partner } \\
\text { eingereichte Anträge }\end{array}$ & $\begin{array}{c}\text { Anzahl Partner } \\
\text { genehmigte Anträge }\end{array}$ & $\begin{array}{c}\text { Anteil Partner } \\
\text { genehmigt (\%) }\end{array}$ \\
\hline IKT der Zukunft & $\mathbf{9 5 1}$ & $\mathbf{3 3 3}$ & $\mathbf{3 5 \%}$ \\
\hline IKT der Zukunft & 906 & 318 & $35 \%$ \\
\hline IKT der Zukunft Dienstleistungen & 42 & 14 & $33 \%$ \\
\hline IKT der Zukunft Professur & 3 & 1 & $33 \%$ \\
\hline Gemeinsame Technologieinitiativen & $\mathbf{3 4 3}$ & $\mathbf{1 5 6}$ & $\mathbf{4 5 \%}$ \\
\hline ARTEMIS & 46 & 27 & $59 \%$ \\
\hline ENIAC & 50 & 32 & $64 \%$ \\
\hline ECSEL & 247 & 97 & $39 \%$ \\
\hline Demographischer Wandel & $\mathbf{8 4 2}$ & $\mathbf{2 8 0}$ & $\mathbf{3 3 \%}$ \\
\hline AAL & 354 & 94 & $27 \%$ \\
\hline benefit & 471 & 182 & $39 \%$ \\
\hline benefit Dienstleistungen & 17 & 4 & $24 \%$ \\
\hline Gesamtzahl Partner & $\mathbf{2 . 1 3 6}$ & $\mathbf{7 6 9}$ & $\mathbf{3 6 \%}$ \\
\hline
\end{tabular}

* bis zum 01.08.2017 entschiedene Anträge; Quelle: FFG, eigene Berechnung inspire research

Insgesamt legen diese Auswertungsergebnisse nahe, dass die IKT-Programme des BMVIT in den vergangenen Jahren deutlich kompetitiver geworden sind. 


\section{Beantragte Projektkosten und Umfang der finanziellen Förderungen}

In welchem finanziellen Umfang wurden in den BMVIT IKT-Programmen seit 2002 Projekte gefördert? Während der Laufzeit des Impulsprogramms FIT-IT wurden Projekte mit einem beantragten Gesamtbudget von mehr 663 Mio. Euro eingereicht. Bei den bewilligten Projekten wurden Kosten von insgesamt rund 281 Mio. Euro als förderfähig anerkannt.

Tabelle 5 Impulsprogramm FIT-IT: beantragte und genehmigte Projektkosten sowie Barwerte der Förderung nach Programmen / Programmlinien 2002-2012 (Mio. Euro)

\begin{tabular}{|c|c|c|c|c|}
\hline Programm / Programmlinie & $\begin{array}{c}\text { Kosten } \\
\text { beantragt }\end{array}$ & $\begin{array}{c}\text { Kosten } \\
\text { genehmigt }\end{array}$ & $\begin{array}{c}\text { Barwert } \\
\text { Förderung }\end{array}$ & $\begin{array}{c}\text { Förder- } \\
\text { quote }\end{array}$ \\
\hline Impulsprogramm FIT-IT & $\mathbf{3 4 0 , 6}$ & $\mathbf{1 4 5 , 6}$ & $\mathbf{9 5 , 2}$ & $\mathbf{6 5 \%}$ \\
\hline Embedded Systems & 109,3 & 43,9 & 28,3 & $65 \%$ \\
\hline Semantic Systems & 60,5 & 24,0 & 17,5 & $73 \%$ \\
\hline Systems on Chip & 34,7 & 22,3 & 12,7 & $57 \%$ \\
\hline Trust in IST & 37,7 & 16,4 & 10,1 & $62 \%$ \\
\hline Visual Computing & 40,5 & 17,5 & 12,6 & $72 \%$ \\
\hline Initiativen \& Joint Call & 57,9 & 21,5 & 14,0 & $65 \%$ \\
\hline Gemeinsame Technologieinitiativen & $\mathbf{2 0 6 , 0}$ & $\mathbf{9 6 , 2}$ & $\mathbf{4 4 , 5}$ & $\mathbf{4 6 \%}$ \\
\hline ARTEMIS & 99,2 & 51,2 & 25,2 & $49 \%$ \\
\hline ENIAC & 106,8 & 44,9 & 19,4 & $43 \%$ \\
\hline Demographischer Wandel & $\mathbf{1 1 6 , 7}$ & $\mathbf{3 9 , 3}$ & $\mathbf{2 5 , 1}$ & $\mathbf{6 4 \%}$ \\
\hline AAL & 79,1 & 23,1 & 15,0 & $65 \%$ \\
\hline benefit & 37,6 & 16,3 & 10,1 & $62 \%$ \\
\hline Gesamt & $\mathbf{6 6 3 , 4}$ & $\mathbf{2 8 1 , 1}$ & $\mathbf{1 6 4 , 8}$ & $\mathbf{5 9 \%}$ \\
\hline Quelle: FFG eigene Berechnung inspire research & & & & \\
\hline
\end{tabular}

Quelle: FFG, eigene Berechnung inspire research

Der Barwert der genehmigten Förderungen betrug in Summe 164,8 Mio. Euro. Davon entfielen 95,2 Mio. Euro (58 \%) auf Projekte der nationalen IKT-Projektförderung, 44,5 Mio. Euro auf österreichische Partner in Projekten der Gemeinsamen Technologieinitiativen (27\%) und 25,1 Mio. Euro auf Projekte im Bereich Demographischer Wandel (15\%). Die durchschnittliche Förderintensität (Förderquote berechnet als Anteil des Barwerts an den genehmigten Gesamtkosten) betrug durchschnittlich $59 \%$.

Seit dem Start der Themeninitiative "IKT der Zukunft" ist die Förderquote bei Projekten der nationalen kooperativen Projektförderung (d. h. ohne Dienstleistungen und Stiftungsprofessuren) um einige Prozentpunkte auf nunmehr $72 \%$ Förderquote angestiegen. Vergleichsweise stabil blieb die Förderquote bei bewilligten Projekten zum Demographischen Wandel.

Die Förderquote für österreichische Teilnehmer an Projekten in den Gemeinsamen Technologieinitiativen verringerte sich von durchschnittlich $46 \%$ während der Laufzeit des Impulsprogramms FIT-IT auf $37 \%$ seit Start der Themeninitiative "IKT der Zukunft". Nach der Zusammenführung von ARTEMIS und ENIAC zu ECSEL betrug die durchschnittliche Förderintensität jedoch wieder $42 \%$. Die insgesamt geringeren Förderquoten in den Programmen der Gemeinsamen Technologieinitiativen spiegeln dabei vor allem den höheren Anteil großer Unternehmen unter den Förderempfängern wider.

Bemerkenswert ist in diesem Zusammenhang der deutlich höhere Budgetanteil von Projektanträgen und geförderten Projekten (bzw. genehmigten Förderungen) in den Gemeinsamen Technologieinitiativen seit Start der Themeninitiative "IKT der Zukunft" im Vergleich zum Durchführungszeitraum des Impulsprogramms FIT-IT. Während der Laufzeit von FIT-IT wur- 
den 27 \% der gesamten Barwerte für Förderungen im Programm für Projekte in den Gemeinsamen Technologieinitiativen gewidmet, während seit dem Start von IKT der Zukunft der Fördermittelanteil von ARTEMIS-, ENIAC- und ECSEL-Projekten an den insgesamt genehmigten Förderungen auf $54 \%$ zunahm.

Tabelle 6 Themeninitiative "IKT der Zukunft": beantragte und genehmigte Projektkosten sowie Barwerte der Förderung nach Förderthemen 2012-2016 (Mio. Euro)

\begin{tabular}{|l|c|c|c|c|}
\hline Förderthemen & $\begin{array}{c}\text { Kosten } \\
\text { beantragt }\end{array}$ & $\begin{array}{c}\text { Kosten } \\
\text { genehmigt }\end{array}$ & $\begin{array}{c}\text { Barwert } \\
\text { Förderung }\end{array}$ & $\begin{array}{c}\text { Förder- } \\
\text { quote }\end{array}$ \\
\hline IKT der Zukunft & $\mathbf{1 8 1 , 7}$ & $\mathbf{5 7 , 8}$ & $\mathbf{4 1 , 3}$ & $\mathbf{7 1 \%}$ \\
\hline IKT der Zukunft & 172,8 & 54,6 & 39,4 & $\mathbf{7 2 \%}$ \\
\hline IKT der Zukunft Dienstleistungen & 1,4 & 0,7 & 0,6 & $92 \%$ \\
\hline IKT der Zukunft Professur & 7,5 & 2,5 & 1,3 & $50 \%$ \\
\hline Gemeinsame Technologieinitiativen & $\mathbf{4 0 6 , 9}$ & $\mathbf{2 3 6 , 4}$ & $\mathbf{8 7 , 5}$ & $\mathbf{3 7 \%}$ \\
\hline ARTEMIS & 43,7 & 33,2 & 13,4 & $40 \%$ \\
\hline ENIAC & 92,5 & 73,5 & 19,7 & $27 \%$ \\
\hline ECSEL & 270,7 & 129,7 & 54,4 & $42 \%$ \\
\hline Demographischer Wandel & $\mathbf{1 6 6 , 5}$ & $\mathbf{5 2 , 1}$ & $\mathbf{3 2 , 5}$ & $\mathbf{6 2 \%}$ \\
\hline AAL & 105,5 & 28,8 & 19,1 & $66 \%$ \\
\hline benefit & 60,6 & 23,2 & 13,3 & $57 \%$ \\
\hline benefit Dienstleistungen & 0,6 & 0,1 & 0,1 & $100 \%$ \\
\hline Gesamt & $\mathbf{7 5 5 , 1}$ & $\mathbf{3 4 6 , 3}$ & $\mathbf{1 6 1 , 3}$ & $\mathbf{4 7 \%}$ \\
\hline
\end{tabular}

" bis zum 01.08.2017 entschiedene Projekte; Quelle: FFG, eigene Berechnung inspire research

Für die folgenden Abbildungen wurden die bewilligten Barwerte der Förderung anteilsmäßig über die Projektlaufzeit (Förderjahre) verteilt und nach Programmen gegliedert aufsummiert. Die Abbildungen verdeutlichen die Zunahme der Förderung von Projekten der Gemeinsamen Technologieinitiativen sowohl absolut als auch relativ zu den anderen Förderschwerpunkten der BMVIT Themeninitiative "IKT der Zukunft".

Abbildung 1 Impulsprogramm FIT-IT und Themeninitiative "IKT der Zukunft": Summe der genehmigten Barwerte verteilt auf die Projektlaufzeiten nach Programmen / Förderthemen (Mio. Euro)

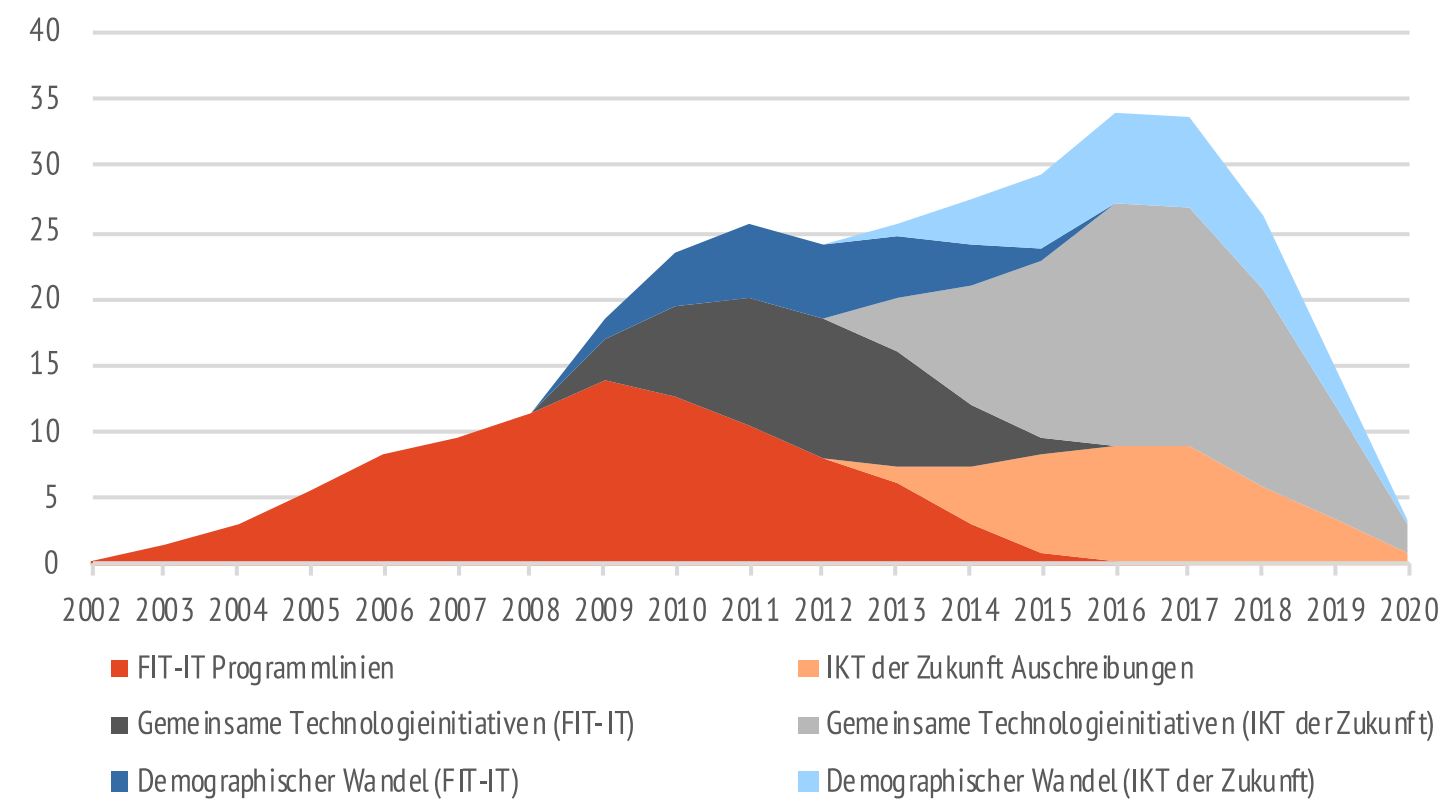

Quelle: FFG, Auswertung und Darstellung inspire research 
Diese Entwicklung ist dabei nicht nur dem Umstand geschuldet, dass Projekte zu den Gemeinsamen Technologieinitiativen (und zum Thema Demographischer Wandel) erst seit 2008 gefördert werden, während die nationale Förderung in FIT-IT bereits 2002 begann.

Abbildung 2 Impulsprogramm FIT-IT und Themeninitiative „IKT der Zukunft": Anteile an der Summe der genehmigten Barwerte verteilt auf die Projektlaufzeiten nach Programmen / Förderthemen

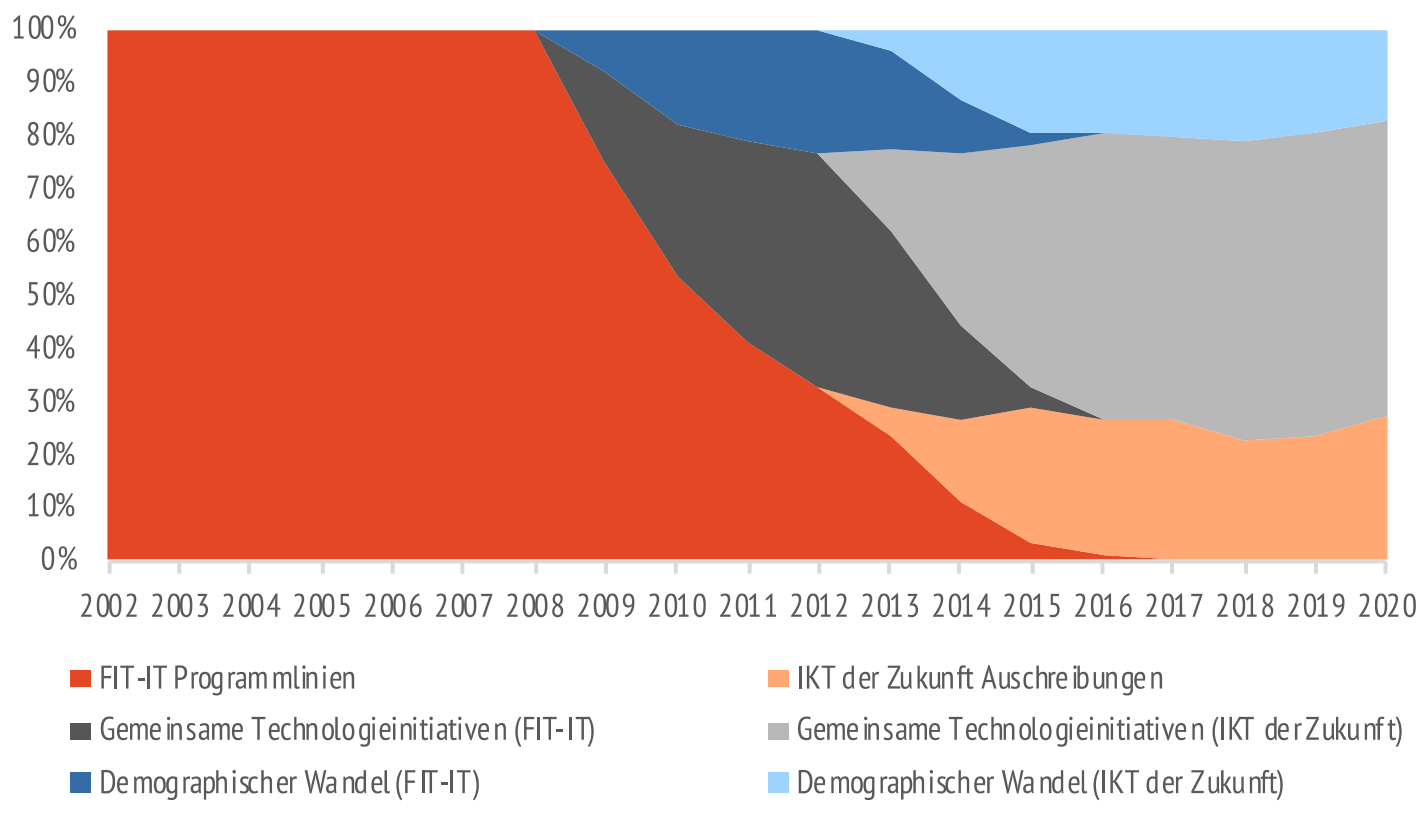

Quelle: FFG, Auswertung und Darstellung inspire research

Wie die Auswertungen zeigen, hat über die Jahre auch anteilsmäßig die budgetäre Bedeutung von Projekten in den Gemeinsamen Technologieinitiativen deutlich zugenommen.

\subsection{Beteiligung nach Teilnehmergruppen}

In welchem Umfang haben sich verschiedene Gruppen von Organisationen am Impulsprogramm FIT-IT und seit 2012 an der Themeninitiative "IKT der Zukunft" beteiligt? Die Auswertung der FFG-Antrags- und Förderdaten zeigt, dass etwa die Hälfte der Antragsteller/innen in FIT-IT aus dem wissenschaftlichen Bereich (d. h. Forschungseinrichtungen und Hochschulen) und die Hälfte aus (gewerblichen) Unternehmen und sonstigen Organisationen (z. B. Verbände, öffentlicher Bereich, NPO / Nicht profitorientierte Betriebe und Einrichtungen, Hilfsorganisationen) kamen. Innerhalb des wissenschaftlichen Bereichs dominierten in den thematischen FIT-IT Programmlinien die Hochschulen, während in Projekten der Gemeinsamen Technologieinitiativen und zum Demographischen Wandel Hochschulen und (außeruniversitäre) Forschungseinrichtungen in ähnlichem Ausmaß beteiligt waren.

Der Anteil an Unternehmen unter den Einreichern war im Einklang mit den Zielsetzungen der Förderung in den Gemeinsamen Technologieinitiativen mit 64 \% besonders hoch. Die Bedeutung von Einrichtungen, die nicht zu den klassischen FuE-Akteuren zählen, zeigt sich in den Programmen zum Demographischen Wandel. Rund 10 \% der Einreicher in AAL- und benefitProjekten gehörte der Gruppe der Sonstigen Einreicher an. In den transnational ausgerichteten Projekten zum Demographischen Wandel waren auch außeruniversitäre Forschungseinrichtungen überproportional beteiligt.

Die Verteilung zwischen den verschiedenen Teilnehmergruppen verändert sich nur wenig, wenn wir nur die Beteiligungen an bewilligten Projektanträgen betrachten. Antragstellende 
Hochschulen waren in den Programmlinien der nationalen FIT-IT-Förderung etwas erfolgreicher als die anderen Antragstellergruppen.

Tabelle 7 Impulsprogramm FIT-IT: Anzahl der Beteiligungen an Projektanträgen nach Teilnehmergruppen 2002-2012

\begin{tabular}{|c|cc|cc|cc|c|c|}
\hline Programm / Programmlinie & \multicolumn{2}{|c|}{$\begin{array}{c}\text { Forschungs- } \\
\text { einricht. }\end{array}$} & \multicolumn{2}{|c|}{$\begin{array}{c}\text { Hoch- } \\
\text { schulen }\end{array}$} & \multicolumn{2}{c|}{$\begin{array}{c}\text { Sonstige } \\
\text { Einreicher }\end{array}$} & \multicolumn{2}{c|}{$\begin{array}{c}\text { Unter- } \\
\text { nehmen }\end{array}$} \\
\hline Impulsprogramm FIT-IT & $\mathbf{2 4 5}$ & $\mathbf{1 4} \%$ & $\mathbf{6 9 1}$ & $\mathbf{3 9} \%$ & $\mathbf{3 6}$ & $\mathbf{2 \%}$ & $\mathbf{7 7 9}$ & $\mathbf{4 4 \%}$ \\
\hline Embedded Systems & 55 & $12 \%$ & 213 & $45 \%$ & 8 & $2 \%$ & 202 & $42 \%$ \\
\hline Semantic Systems & 67 & $14 \%$ & 180 & $39 \%$ & 14 & $3 \%$ & 206 & $44 \%$ \\
\hline Systems on Chip & 2 & $2 \%$ & 48 & $52 \%$ & & $0 \%$ & 42 & $46 \%$ \\
\hline Trust in IST & 22 & $14 \%$ & 54 & $34 \%$ & 1 & $1 \%$ & 84 & $52 \%$ \\
\hline Visual Computing & 47 & $18 \%$ & 97 & $38 \%$ & 2 & $1 \%$ & 111 & $43 \%$ \\
\hline Initiativen \& Joint Call & 52 & $18 \%$ & 99 & $33 \%$ & 11 & $4 \%$ & 134 & $45 \%$ \\
\hline Gemeinsame Technologieinitiativen & $\mathbf{3 5}$ & $\mathbf{1 7} \%$ & $\mathbf{3 7}$ & $\mathbf{1 8 \%}$ & $\mathbf{3}$ & $\mathbf{1 \%}$ & $\mathbf{1 3 2}$ & $\mathbf{6 4 \%}$ \\
\hline ARTEMIS & 28 & $\mathbf{2 5} \%$ & 12 & $11 \%$ & 3 & $3 \%$ & 67 & $61 \%$ \\
\hline ENIAC & 7 & $7 \%$ & 25 & $26 \%$ & & $0 \%$ & 65 & $67 \%$ \\
\hline Demographischer Wandel & $\mathbf{1 2 8}$ & $\mathbf{2 3} \%$ & $\mathbf{1 1 5}$ & $\mathbf{2 1 \%}$ & $\mathbf{5 4}$ & $\mathbf{1 0 \%}$ & $\mathbf{2 5 9}$ & $\mathbf{4 7 \%}$ \\
\hline AAL & 65 & $31 \%$ & 44 & $21 \%$ & 20 & $9 \%$ & 82 & $39 \%$ \\
\hline benefit & 63 & $18 \%$ & 71 & $21 \%$ & 34 & $10 \%$ & 177 & $51 \%$ \\
\hline Gesamt & $\mathbf{4 0 8}$ & $\mathbf{1 6} \%$ & $\mathbf{8 4 3}$ & $\mathbf{3 4} \%$ & $\mathbf{9 3}$ & $\mathbf{4} \%$ & $\mathbf{1 . 1 7 0}$ & $\mathbf{4 7 \%}$ \\
\hline
\end{tabular}

Quelle: FFG, eigene Berechnung inspire research

Die nicht-klassischen FuE-Akteure in den Programmen zum Demographischen Wandel hatten insbesondere im transnationalen AAL-Programm schlechtere Erfolgsaussichten auf die Genehmigung ihrer Anträge, während im nationalen Programm benefit ihr Anteil unter den Geförderten in etwa auch ihrem Anteil unter den insgesamt einreichenden Einrichtungen entsprach.

Tabelle 8 Impulsprogramm FIT-IT: Anzahl der Beteiligungen an genehmigten Projektanträgen nach Teilnehmergruppen 2002-2012

\begin{tabular}{|c|c|c|c|c|c|c|c|c|}
\hline \multirow{2}{*}{$\begin{array}{l}\text { Programm / Programmlinie } \\
\text { Impulsprogramm FIT-IT }\end{array}$} & \multicolumn{2}{|c|}{$\begin{array}{l}\text { Forschungs- } \\
\text { einricht. }\end{array}$} & \multicolumn{2}{|c|}{$\begin{array}{l}\text { Hoch- } \\
\text { schulen }\end{array}$} & \multicolumn{2}{|c|}{$\begin{array}{l}\text { Sonstige } \\
\text { Einreicher }\end{array}$} & \multicolumn{2}{|c|}{$\begin{array}{c}\text { Unter- } \\
\text { nehmen }\end{array}$} \\
\hline & 101 & $13 \%$ & 333 & $42 \%$ & 16 & $2 \%$ & 339 & $43 \%$ \\
\hline Embedded Systems & 23 & $10 \%$ & 112 & $50 \%$ & 6 & $3 \%$ & 85 & $38 \%$ \\
\hline Semantic Systems & 26 & $13 \%$ & 80 & $40 \%$ & 5 & $2 \%$ & 91 & $45 \%$ \\
\hline Systems on Chip & 2 & $4 \%$ & 28 & $52 \%$ & - & $0 \%$ & 24 & $44 \%$ \\
\hline Trust in IST & 7 & $11 \%$ & 21 & $34 \%$ & - & $0 \%$ & 33 & $54 \%$ \\
\hline Visual Computing & 21 & $18 \%$ & 49 & $42 \%$ & - & $0 \%$ & 47 & $40 \%$ \\
\hline Initiativen \& Joint Call & 22 & $17 \%$ & 43 & $33 \%$ & 5 & $4 \%$ & 59 & $46 \%$ \\
\hline Gemeinsame Technologieinitiativen & 20 & $20 \%$ & 18 & $18 \%$ & - & $0 \%$ & 63 & $62 \%$ \\
\hline ARTEMIS & 14 & $27 \%$ & 5 & $10 \%$ & - & $0 \%$ & 32 & $63 \%$ \\
\hline ENIAC & 6 & $12 \%$ & 13 & $26 \%$ & - & $0 \%$ & 31 & $62 \%$ \\
\hline Demographischer Wandel & 65 & $24 \%$ & 57 & $21 \%$ & 20 & $7 \%$ & 130 & $48 \%$ \\
\hline AAL & 26 & $35 \%$ & 14 & $19 \%$ & 3 & $4 \%$ & 31 & $42 \%$ \\
\hline benefit & 39 & $20 \%$ & 43 & $22 \%$ & 17 & $9 \%$ & 99 & $50 \%$ \\
\hline Gesamt & 186 & $16 \%$ & 408 & $35 \%$ & 36 & $3 \%$ & 532 & $46 \%$ \\
\hline
\end{tabular}

Bezogen auf die genehmigten Förderungen zeigt die Auswertung der FFG-Daten, dass während der Laufzeit des Impulsprogramms FIT-IT etwas weniger als die Hälfte (48\%) der Bar- 
werte der Förderungen an Unternehmen ausbezahlt wurden. In den auch inhaltlich klar industrieorientierten Gemeinsamen Technologieinitiativen lag der Fördermittelanteil der Unternehmen bei $72 \%$. Am geringsten war der Fördermittelanteil der Unternehmen in den Förderlinien Semantic Systems (23\%) und Visual Computing (26\%).

Tabelle 9 Impulsprogram FIT-IT: Förderbarwerte der genehmigten Projektanträge nach Teilnehmergruppen 2002-2012 (Mio. Euro)

\begin{tabular}{|c|c|c|c|c|c|c|c|c|}
\hline \multirow{2}{*}{$\begin{array}{l}\text { Programm / Programmlinie } \\
\text { Impulsprogramm FIT-IT } \\
\end{array}$} & \multicolumn{2}{|c|}{$\begin{array}{l}\text { Forschungs- } \\
\text { einricht. }\end{array}$} & \multicolumn{2}{|c|}{$\begin{array}{l}\text { Hoch- } \\
\text { schulen }\end{array}$} & \multicolumn{2}{|c|}{$\begin{array}{l}\text { Sonstige } \\
\text { Einreicher }\end{array}$} & \multicolumn{2}{|c|}{$\begin{array}{c}\text { Unter- } \\
\text { nehmen }\end{array}$} \\
\hline & 17,6 & $18 \%$ & 40,4 & $42 \%$ & 0,5 & $1 \%$ & 36,7 & $39 \%$ \\
\hline Embedded Systems & 4.3 & $15 \%$ & 12,7 & $45 \%$ & 0,2 & $1 \%$ & 11,1 & $39 \%$ \\
\hline Semantic Systems & 4,6 & $26 \%$ & 8,9 & $51 \%$ & 0,0 & $0 \%$ & 4,0 & $23 \%$ \\
\hline Systems on Chip & 0,5 & $4 \%$ & 4,2 & $33 \%$ & 0,0 & $0 \%$ & 8,0 & $63 \%$ \\
\hline Trust in IST & 1,2 & $12 \%$ & 3.7 & $36 \%$ & 0,0 & $0 \%$ & 5.3 & $52 \%$ \\
\hline Visual Computing & 3,0 & $24 \%$ & 6,4 & $51 \%$ & 0,0 & $0 \%$ & 3,2 & $26 \%$ \\
\hline Initiativen \& Joint Call & 4,1 & $29 \%$ & 4,6 & $33 \%$ & 0,3 & $2 \%$ & 5,1 & $36 \%$ \\
\hline Gemeinsame Technologieinitiativen & 7,2 & $16 \%$ & 5,4 & $12 \%$ & 0,0 & $0 \%$ & 31,9 & $72 \%$ \\
\hline ARTEMIS & 5,8 & $23 \%$ & 2,0 & $8 \%$ & 0,0 & $0 \%$ & 17,4 & $69 \%$ \\
\hline ENIAC & 1,4 & $7 \%$ & 3,4 & $17 \%$ & 0,0 & $0 \%$ & 14,6 & $75 \%$ \\
\hline Demographischer Wandel & 9,0 & $36 \%$ & 5,5 & $22 \%$ & 0,7 & $3 \%$ & 9,9 & $39 \%$ \\
\hline AAL & 6,4 & $42 \%$ & 2,8 & $19 \%$ & 0,2 & $1 \%$ & 5,6 & $37 \%$ \\
\hline benefit & 2,6 & $26 \%$ & 2,6 & $26 \%$ & 0,5 & $5 \%$ & 4,3 & $42 \%$ \\
\hline Gesamt & 33,8 & $21 \%$ & 51,3 & $31 \%$ & 1,2 & $1 \%$ & 78,5 & $48 \%$ \\
\hline
\end{tabular}

Ein Vergleich der Beteiligungszahlen nach Teilnehmergruppen zwischen dem Impulsprogramm FIT-IT und der Themeninitiative IKT der Zukunft zeigt, dass insbesondere die Beteiligung der Hochschulen deutlich zurückgegangen ist. Dies betrifft insbesondere die nationale kooperative FuE-Förderung. Während der Anteil der Hochschulen unter den Einreichern über die FIT-IT Programmlinien hinweg noch $39 \%$ ausmachte, waren es in den bisherigen Ausschreibungen von IKT der Zukunft nur mehr $27 \%$. Erhöht hat sich im Gegenzug insbesondere der Anteil der außeruniversitären Forschungseinrichtungen unter den Einreichern.

Tabelle 10 Themeninitiative „IKT der Zukunft": Anzahl der Beteiligungen an Projektanträgen nach Teilnehmergruppen $2012-2016^{*}$

\begin{tabular}{|l|cc|cc|cc|c|c|}
\hline Förderthemen & \multicolumn{2}{|c|}{$\begin{array}{c}\text { Forschungs- } \\
\text { einricht. }\end{array}$} & \multicolumn{2}{|c|}{$\begin{array}{c}\text { Hoch- } \\
\text { schulen }\end{array}$} & \multicolumn{2}{c|}{$\begin{array}{c}\text { Sonstige } \\
\text { Einreicher }\end{array}$} & $\begin{array}{c}\text { Unter- } \\
\text { nehmen }\end{array}$ \\
\hline IKT der Zukunft & $\mathbf{2 1 6}$ & $\mathbf{2 3} \%$ & $\mathbf{2 5 7}$ & $\mathbf{2 7} \%$ & $\mathbf{2 5}$ & $\mathbf{3 \%}$ & $\mathbf{4 5 3}$ & $\mathbf{4 8 \%}$ \\
\hline IKT der Zukunft & 199 & $22 \%$ & 244 & $27 \%$ & 22 & $2 \%$ & 441 & $49 \%$ \\
\hline IKTdZ Dienstleistungen & 17 & $40 \%$ & 10 & $24 \%$ & 3 & $7 \%$ & 12 & $29 \%$ \\
\hline IKTdZ Professur & & - & 3 & $100 \%$ & & - & & - \\
\hline Gemeinsame Technologieinitiativen & $\mathbf{8 1}$ & $\mathbf{2 4} \%$ & $\mathbf{5 9}$ & $\mathbf{1 7} \%$ & & - & $\mathbf{2 0 3}$ & $\mathbf{5 9 \%}$ \\
\hline ARTEMIS & 12 & $26 \%$ & 9 & $20 \%$ & & - & 25 & $54 \%$ \\
\hline ENIAC & 11 & $22 \%$ & 9 & $18 \%$ & & - & 30 & $60 \%$ \\
\hline ECSEL & 58 & $23 \%$ & 41 & $17 \%$ & & - & 148 & $60 \%$ \\
\hline Demographischer Wandel & $\mathbf{1 8 1}$ & $\mathbf{2 1 \%}$ & $\mathbf{1 8 3}$ & $\mathbf{2 2 \%}$ & $\mathbf{1 4 6}$ & $\mathbf{1 7 \%}$ & $\mathbf{3 3 2}$ & $\mathbf{3 9 \%}$ \\
\hline AAL & 89 & $25 \%$ & 74 & $21 \%$ & 59 & $17 \%$ & 132 & $37 \%$ \\
\hline benefit & 89 & $19 \%$ & 105 & $22 \%$ & 80 & $17 \%$ & 197 & $42 \%$ \\
\hline benefit Dienstleistungen & 3 & $18 \%$ & 4 & $24 \%$ & 7 & $41 \%$ & 3 & $18 \%$ \\
\hline Gesamt & $\mathbf{4 7 8}$ & $\mathbf{2 2} \%$ & $\mathbf{4 9 9}$ & $\mathbf{2 3 \%}$ & $\mathbf{1 7 1}$ & $\mathbf{8 \%}$ & $\mathbf{9 8 8}$ & $\mathbf{4 6 \%}$ \\
\hline
\end{tabular}

" bis zum 01.08.2017 entschiedene Projekte; Quelle: FFG, eigene Berechnung inspire research 
In den Projekten zum Demographischen Wandel ist im Einklang mit den Förderzielen der Anteil der Antragsteller, die nicht zur Gruppe der wissenschaftlichen Einrichtungen und der (gewerblichen) Unternehmen zählen weiter angestiegen. Auch ihr Anteil unter den Geförderten entspricht nunmehr in etwa jenem unter den Antragstellern.

Tabelle 11 Themeninitiative „IKT der Zukunft": Anzahl der Beteiligungen an genehmigten Projektanträgen nach Teilnehmergruppen $2012-2016^{*}$

\begin{tabular}{|l|cc|cc|cc|cc|}
\hline Förderthemen & \multicolumn{2}{|c|}{$\begin{array}{c}\text { Forschungs- } \\
\text { einricht. }\end{array}$} & \multicolumn{2}{|c|}{$\begin{array}{c}\text { Hoch- } \\
\text { schulen }\end{array}$} & \multicolumn{2}{c|}{$\begin{array}{c}\text { Sonstige } \\
\text { Einreicher }\end{array}$} & \multicolumn{2}{c|}{$\begin{array}{c}\text { Unter- } \\
\text { nehmen }\end{array}$} \\
\hline IKT der Zukunft & $\mathbf{7 8}$ & $\mathbf{2 3} \%$ & $\mathbf{9 9}$ & $\mathbf{3 0} \%$ & $\mathbf{1 0}$ & $\mathbf{3 \%}$ & $\mathbf{1 4 6}$ & $\mathbf{4 4 \%}$ \\
\hline IKT der Zukunft & 71 & $22 \%$ & 95 & $30 \%$ & 9 & $3 \%$ & 143 & $45 \%$ \\
\hline IKTdZ Dienstleistungen & 7 & $50 \%$ & 3 & $21 \%$ & 1 & $7 \%$ & 3 & $21 \%$ \\
\hline IKTdZ Professur & & - & 1 & $100 \%$ & - & - & - & - \\
\hline Gemeinsame Technologieinitiativen & $\mathbf{4 4}$ & $\mathbf{2 8 \%}$ & $\mathbf{2 9}$ & $\mathbf{1 9} \%$ & - & - & $\mathbf{8 3}$ & $\mathbf{5 3} \%$ \\
\hline ARTEMIS & 8 & $30 \%$ & 7 & $26 \%$ & - & - & 12 & $44 \%$ \\
\hline ENIAC & 8 & $25 \%$ & 4 & $13 \%$ & - & - & 20 & $63 \%$ \\
\hline ECSEL & 28 & $29 \%$ & 18 & $19 \%$ & - & - & 51 & $53 \%$ \\
\hline Demographischer Wandel & $\mathbf{5 8}$ & $\mathbf{2 1 \%}$ & $\mathbf{6 3}$ & $\mathbf{2 3 \%}$ & $\mathbf{4 6}$ & $\mathbf{1 6 \%}$ & $\mathbf{1 1 3}$ & $\mathbf{4 0 \%}$ \\
\hline AAL & 21 & $22 \%$ & 22 & $23 \%$ & 15 & $16 \%$ & 36 & $38 \%$ \\
\hline benefit & 35 & $19 \%$ & 41 & $23 \%$ & 30 & $16 \%$ & 76 & $42 \%$ \\
\hline benefit Dienstleistungen & 2 & $50 \%$ & - & - & 1 & $25 \%$ & 1 & $25 \%$ \\
\hline Gesamt & $\mathbf{1 8 0}$ & $\mathbf{2 3 \%}$ & $\mathbf{1 9 1}$ & $\mathbf{2 5 \%}$ & $\mathbf{5 6}$ & $\mathbf{7 \%}$ & $\mathbf{3 4 2}$ & $\mathbf{4 4 \%}$ \\
\hline
\end{tabular}

* bis zum 01.08.2017 entschiedene Projekte; Quelle: FFG, eigene Berechnung inspire research

Mit dem geringeren Anteil an Beteiligungen an den Einreichungen und Genehmigungen von Anträgen durch Hochschulen ist auch ihr Anteil an den vergebenen Fördermitteln zurückgegangen. Aus den bisherigen nationalen Ausschreibungen von IKT der Zukunft erhielten Hochschulen nur mehr unwesentlich mehr Fördermittel (13,9 Mio. Euro) als die außeruniversitären Forschungseinrichtungen (13,2 Mio. Euro). Auch aus den Projekten in den Gemeinsamen Technologieinitiativen erhielten Hochschulen seit Start der Themeninitiative IKT der Zukunft einen deutlich geringeren Fördermittelanteil als während der Laufzeit des Impulsprogramms FIT-IT.

Tabelle 12 Themeninitiative „IKT der Zukunft“: Förderbarwerte (Mio. Euro) nach Teilnehmergruppen 2012-2016*

\begin{tabular}{|l|cc|cc|cc|cc|}
\hline Förderthemen & \multicolumn{2}{|c|}{$\begin{array}{c}\text { Forschungs- } \\
\text { einricht. }\end{array}$} & \multicolumn{2}{|c|}{$\begin{array}{c}\text { Hoch- } \\
\text { schulen }\end{array}$} & \multicolumn{2}{c|}{$\begin{array}{c}\text { Sonstige } \\
\text { Einreicher }\end{array}$} & \multicolumn{2}{c|}{$\begin{array}{c}\text { Unter- } \\
\text { nehmen }\end{array}$} \\
\hline IKT der Zukunft & $\mathbf{1 3 , 2}$ & $\mathbf{3 2} \%$ & $\mathbf{1 3 , 9}$ & $\mathbf{3 4} \%$ & $\mathbf{0 , 4}$ & $\mathbf{1} \%$ & $\mathbf{1 3 , 9}$ & $\mathbf{3 4 \%}$ \\
\hline IKT der Zukunft & 12,8 & $33 \%$ & 12,5 & $32 \%$ & 0,3 & $1 \%$ & 13,7 & $35 \%$ \\
\hline IKT der Zukunft Dienstleistungen & 0,4 & $59 \%$ & 0,1 & $12 \%$ & 0,0 & $6 \%$ & 0,1 & $23 \%$ \\
\hline IKT der Zukunft Professur & - & - & 1,3 & $100 \%$ & - & - & - & - \\
\hline Gemeinsame Technologieinitiativen & $\mathbf{2 0 , 8}$ & $\mathbf{2 4 \%}$ & $\mathbf{8 , 6}$ & $\mathbf{1 0} \%$ & - & - & $\mathbf{5 8 , 0}$ & $\mathbf{6 6 \%}$ \\
\hline ARTEMIS & 3,8 & $29 \%$ & 1,1 & $8 \%$ & - & - & 8,5 & $63 \%$ \\
\hline ENIAC & 3,5 & $18 \%$ & 1,3 & $6 \%$ & - & - & 15,0 & $76 \%$ \\
\hline ECSEL & 13,6 & $25 \%$ & 6,3 & $12 \%$ & - & - & 34,5 & $64 \%$ \\
\hline Demographischer Wandel & $\mathbf{9 , 3}$ & $\mathbf{2 9} \%$ & $\mathbf{9 , 7}$ & $\mathbf{3 0 \%}$ & $\mathbf{2 , 6}$ & $\mathbf{8 \%}$ & $\mathbf{1 0 , 8}$ & $\mathbf{3 3 \%}$ \\
\hline AAL & 5,6 & $29 \%$ & 6,2 & $33 \%$ & 1,1 & $6 \%$ & 6,1 & $32 \%$ \\
\hline benefit & 3,7 & $28 \%$ & 3,6 & $27 \%$ & 1,4 & $11 \%$ & 4,6 & $35 \%$ \\
\hline benefit Dienstleistungen & $<0,1$ & $35 \%$ & - & - & $<0,1$ & $25 \%$ & $<0,1$ & $39 \%$ \\
\hline Gesamt & $\mathbf{4 3 , 4}$ & $\mathbf{2 7} \%$ & $\mathbf{3 2 , 2}$ & $\mathbf{2 0} \%$ & $\mathbf{3 , 0}$ & $\mathbf{2 \%}$ & $\mathbf{8 2 , 7}$ & $\mathbf{5 1 \%}$ \\
\hline
\end{tabular}

"bis zum 01.08.2017 entschiedene Projekte; Quelle: FFG, eigene Berechnung inspire research 
Der Anteil an Fördermitteln, der an Unternehmen ausbezahlt wurde, liegt bisher in der Themeninitiative "IKT der Zukunft" über dem Vergleichswert im Impulsprogramm FIT-IT, obwohl der Fördermittelanteil der Unternehmen in allen drei Programmteilen abgenommen hat. Dies ist ein Effekt des höheren Anteils der Förderungen für Projekte der Gemeinsamen Technologieinitiativen an den insgesamt vergebenen Förderungen in der Themeninitiative "IKT der Zukunft" im Vergleich zum Impulsprogramm "FIT-IT".

Wir haben diese Veränderungen auch wieder graphisch über die einzelnen Förderjahre der genehmigten Projekte dargestellt. Die folgenden beiden Abbildungen zeigen die Summen bzw. Summenanteile der genehmigten Barwerte der Förderung anteilsmäßig über die Projektlaufzeit (Förderjahre) verteilt nach Gruppen von Antragstellern.

Der Unternehmensanteil an den genehmigten Förderungen hat mit dem Start der Gemeinsamen Technologieinitiativen im Jahr 2008 sehr deutlich zugenommen. Das Maximum des Unternehmensanteils an den Förderungen lag dabei bei Projekten, die in den Jahren 2010 und 2011 durchgeführt wurden. Seither hat der Unternehmensanteil an den Fördermitteln verteilt auf die Projektlaufzeiten wieder geringfügig abgenommen.

Abbildung 3 Summe der Barwerte der Förderung verteilt auf die Projektlaufzeit nach Teilnehmergruppen (Mio. Euro)

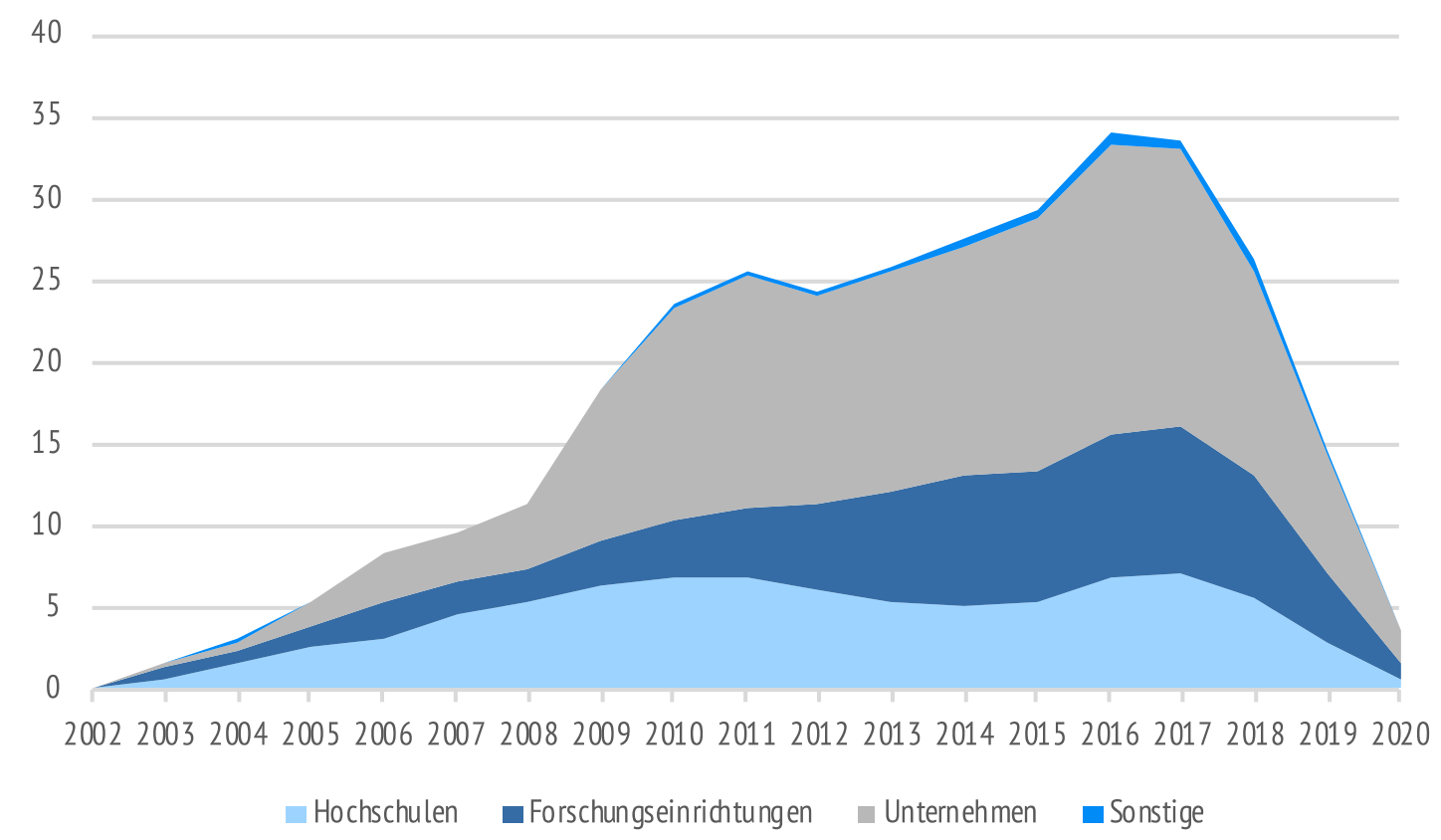

Quelle: FFG, Auswertung und Darstellung inspire research

Der Rückgang der für Hochschulen genehmigten Mittel zeigt sich insbesondere für Projekte, die zwischen 2011 und 2015 durchgeführt wurden. In diesen Jahren nahmen die summierten, auf die Projektlaufzeit umgelegten Förderungen auch nominal ab. Der Fördermittelanteil der Hochschulen an den in den jeweiligen Jahren genehmigten Projekten war jedoch bereits seit dem Jahr 2008 rückläufig. 
Abbildung 4 Anteile an der Summe der Barwerte der Förderung verteilt auf die Projektlaufzeit nach Teilnehmergruppen

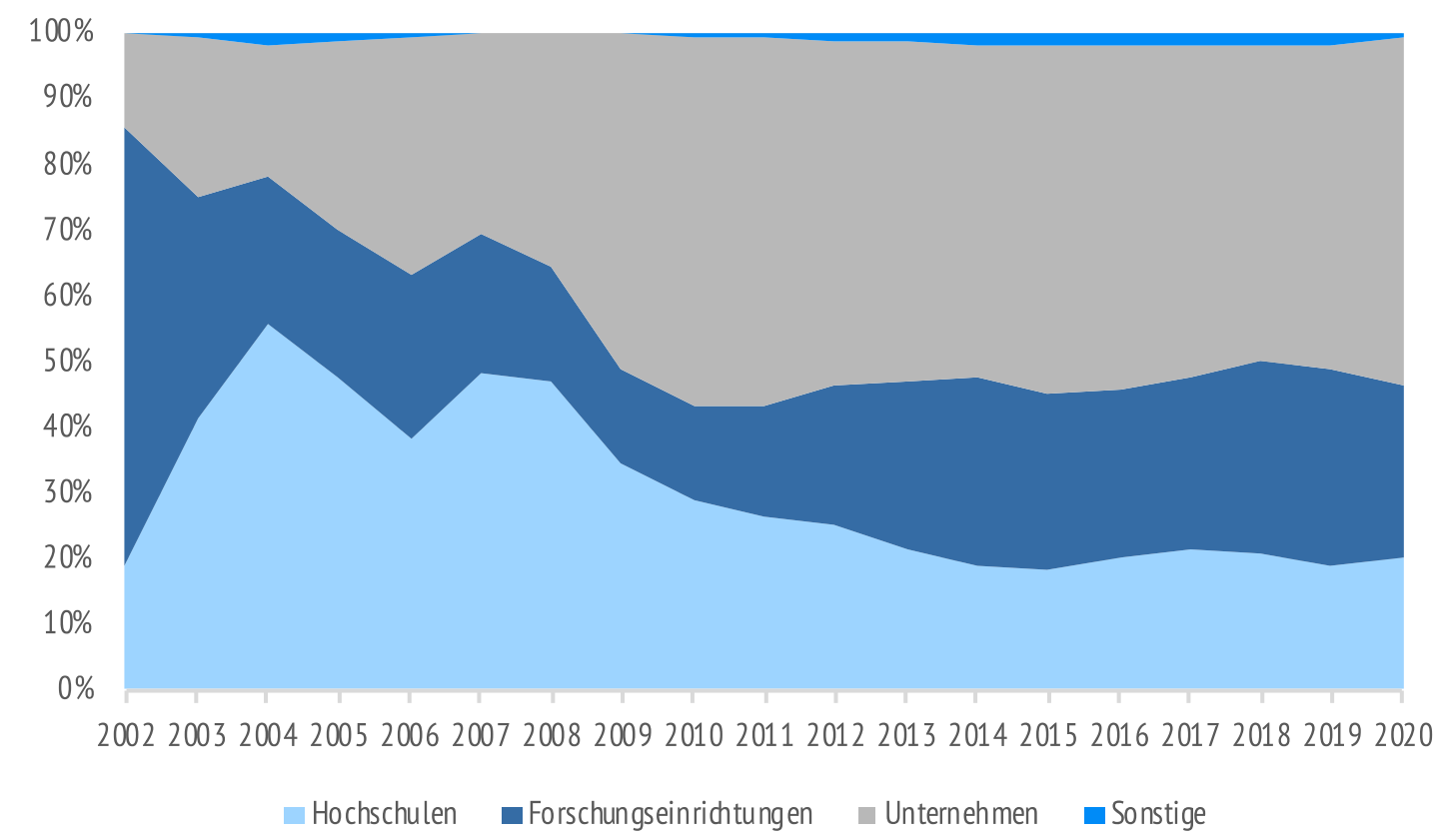

Quelle: FFG, Auswertung und Darstellung inspire research

Reiht man die an den BMVIT IKT-Programmen seit 2002 beteiligten Einrichtungen nach Höhe der bewilligten Förderungen (Förderbarwert), finden sich in der Liste der 25 Einrichtungen mit den höchsten Förderungen 11 Unternehmen und 14 wissenschaftliche Einrichtungen (Universitäten, außeruniversitäre Forschungseinrichtungen, COMET-Zentren, Fachhochschulen).

Abbildung 5 Ranking der Top25-Förderempfänger in den BMVIT IKT-Programmen 2002-2016 (Gesamtsumme der Förderbarwerte in Mio. Euro)

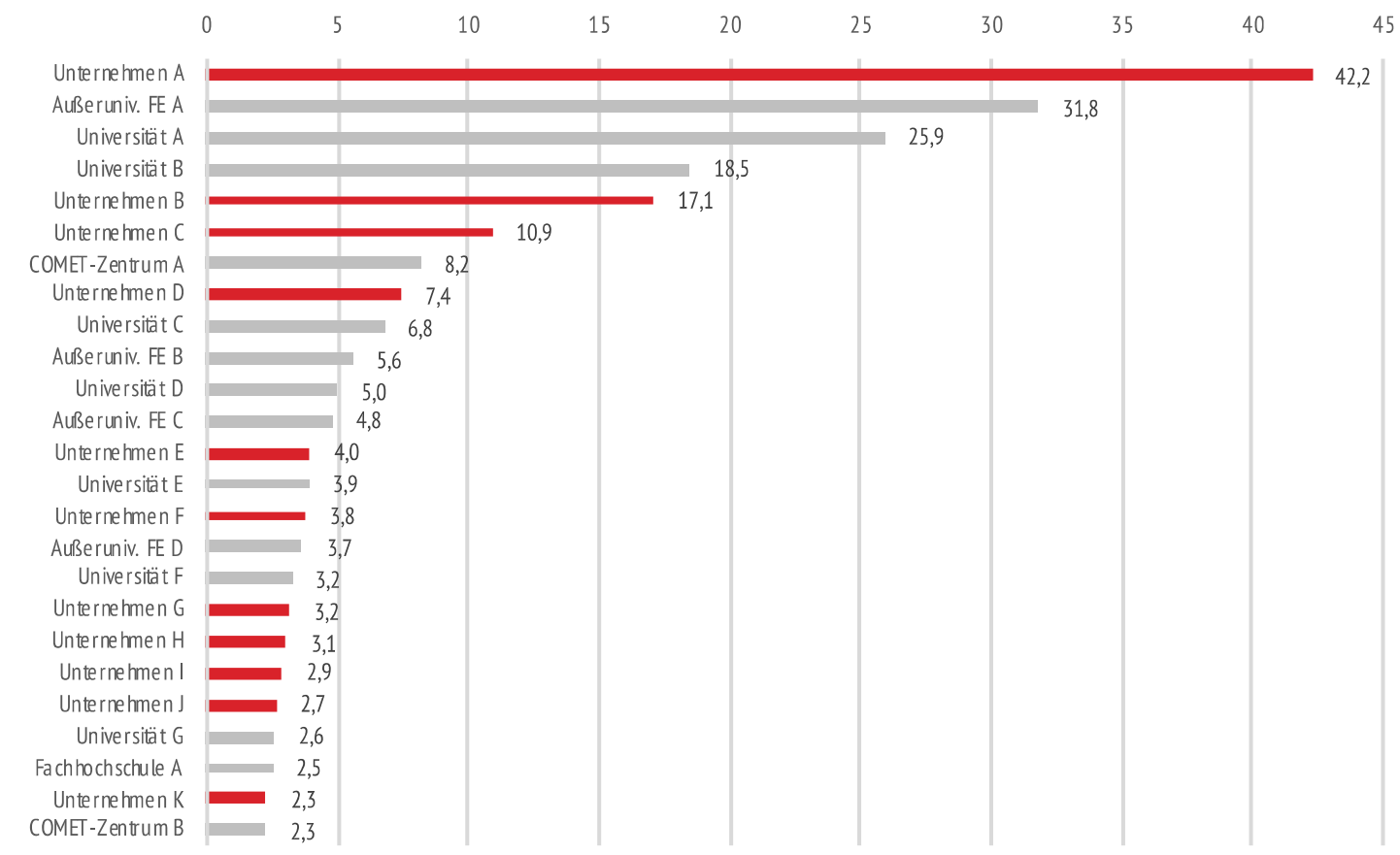

Quelle: FFG, Auswertung und Darstellung inspire research 
Der Anteil der kumulierten Förderungen für diese Gruppe von 25 Einrichtungen an den insgesamt genehmigten Förderungen in den BMVIT IKT-Programmen seit 2002 liegt bei $69 \%$.

\subsection{Beteiligung von Klein- und Mittelunternehmen (KMU)}

Betrachten wir das Ausmaß der Beteiligung von Klein- und Mittelunternehmen (KMU) während der Laufzeit des Impulsprogramms FIT-IT zeigen sich besonders hohe KMU-Anteile unter den Einreichungen von Projekten zum Demographischen Wandel, aber auch in der FITIT Programmlinie Semantic Systems.

KMU hatten insgesamt etwas geringer Erfolgsaussichten, ihre Anträge auch genehmigt zu bekommen, als die Großunternehmen unter den Einreichern. Besonders deutlich war dieser Selektionseffekt in den Gemeinsamen Technologieinitiativen ARTEMIS und ENIAC. Der KMUAnteil unter den antragstellenden Unternehmen ist hier doppelt so hoch wie der KMU-Anteil unter den Unternehmen mit bewilligten Projekten. Nur im Programm benefit und in den FITIT Programmlinien Semantic Systems und Systems on Chip war die Förderwahrscheinlichkeit von KMU praktisch gleich hoch wie jene der Großunternehmen.

Gemessen an den Barwerten erhielten KMU rund 30 \% der insgesamt für Unternehmen genehmigten Fördermittel, wobei zwischen den drei Programmen die Unterschiede sehr groß sind. In ARTEMIS- und ENIAC-Projekten lag der KMU-Barwertanteil an den Unternehmensförderungen bei $11 \%$, in den Projekten zum Demographischen Wandel hingegen bei $80 \%$.

Tabelle 13 Impulsprogramm FIT-IT 2002-2012: KMU-Anteile an den Beteiligungen von Unternehmen (in \%)

\begin{tabular}{|c|c|c|c|c|c|c|}
\hline Programm / Programmlinie & $\begin{array}{l}\text { KMU- } \\
\text { Antrag- } \\
\text { steller }\end{array}$ & $\begin{array}{l}\text { KMU- } \\
\text { Förder- } \\
\text { empf. }\end{array}$ & $\begin{array}{l}\text { KMU- } \\
\text { Kosten- } \\
\text { anteil }\end{array}$ & $\begin{array}{c}\text { KMU- } \\
\text { Anteil } \\
\text { Barwert }\end{array}$ & $\begin{array}{c}\text { Förder- } \\
\text { quote } \\
\text { KMU }\end{array}$ & $\begin{array}{c}\text { Förder- } \\
\text { quote } \\
\text { GU }\end{array}$ \\
\hline Impulsprogramm FIT-IT & $64 \%$ & $57 \%$ & $27 \%$ & $32 \%$ & $68 \%$ & $54 \%$ \\
\hline Embedded Systems & $51 \%$ & $45 \%$ & $18 \%$ & $21 \%$ & $67 \%$ & $54 \%$ \\
\hline Semantic Systems & $85 \%$ & $84 \%$ & $73 \%$ & $75 \%$ & $70 \%$ & $62 \%$ \\
\hline Systems on Chip & $19 \%$ & $17 \%$ & $10 \%$ & $10 \%$ & $57 \%$ & $53 \%$ \\
\hline Trust in IST & $65 \%$ & $58 \%$ & $26 \%$ & $31 \%$ & $69 \%$ & $54 \%$ \\
\hline Visual Computing & $71 \%$ & $57 \%$ & $51 \%$ & $55 \%$ & $73 \%$ & $62 \%$ \\
\hline Initiativen \& Joint Call & $57 \%$ & $47 \%$ & $38 \%$ & $45 \%$ & $69 \%$ & $51 \%$ \\
\hline Gemeinsame Technologieinitiativen & $33 \%$ & $16 \%$ & $9 \%$ & $11 \%$ & $53 \%$ & $39 \%$ \\
\hline ARTEMIS & $40 \%$ & $16 \%$ & $7 \%$ & $10 \%$ & $59 \%$ & $42 \%$ \\
\hline ENIAC & $25 \%$ & $16 \%$ & $10 \%$ & $12 \%$ & $48 \%$ & $37 \%$ \\
\hline Demographischer Wandel & $81 \%$ & $78 \%$ & $74 \%$ & $80 \%$ & $60 \%$ & $42 \%$ \\
\hline AAL & $79 \%$ & $68 \%$ & $69 \%$ & $76 \%$ & $59 \%$ & $41 \%$ \\
\hline benefit & $82 \%$ & $82 \%$ & $80 \%$ & $85 \%$ & $61 \%$ & $42 \%$ \\
\hline Gesamt & $64 \%$ & $57 \%$ & $23 \%$ & $30 \%$ & $63 \%$ & $45 \%$ \\
\hline
\end{tabular}

Quelle: FFG, eigene Berechnung inspire research

Über die gesamte Laufzeit von FIT-IT errechnet sich eine durchschnittliche Förderquote für KMU von $63 \%$ im Vergleich zu einer durchschnittlichen Förderquote von 45 \% für die beteiligten Großunternehmen.

Vergleicht man dazu die Zahlen für die Themeninitiative IKT der Zukunft, fällt auf, dass der Kosten- und Barwertanteil der KMU an den genehmigten Projekten von Unternehmen in der nationalen kooperativen FuE-Förderung deutlich zugenommen hat. Der Unterschied in den bewilligten Projektgrößen zwischen KMU und Großunternehmen hat also abgenommen. 
Tabelle 14 BMVIT Themeninitiative „IKT der Zukunft" 2012-2016*: KMU-Anteile an den Beteiligungen von Unternehmen (in \%)

\begin{tabular}{|c|c|c|c|c|c|c|}
\hline $\begin{array}{l}\text { Themeninitiative } \\
\text { "IKT der Zukunft" }\end{array}$ & $\begin{array}{l}\text { KMU- } \\
\text { Antrag- } \\
\text { steller }\end{array}$ & $\begin{array}{l}\text { KMU- } \\
\text { Förder- } \\
\text { empf. }\end{array}$ & $\begin{array}{l}\text { KMU- } \\
\text { Kosten- } \\
\text { anteil }\end{array}$ & $\begin{array}{c}\text { KMU- } \\
\text { Anteil } \\
\text { Barwert }\end{array}$ & $\begin{array}{c}\text { Förder- } \\
\text { quote } \\
\text { KMU }\end{array}$ & $\begin{array}{c}\text { Förder- } \\
\text { quote } \\
\text { GU }\end{array}$ \\
\hline IKT der Zukunft & $62 \%$ & $55 \%$ & $43 \%$ & $50 \%$ & $72 \%$ & $54 \%$ \\
\hline IKT der Zukunft & $61 \%$ & $55 \%$ & $43 \%$ & $50 \%$ & $72 \%$ & $54 \%$ \\
\hline IKT der Zukunft Dienstleistungen & $84 \%$ & $33 \%$ & $42 \%$ & $42 \%$ & $100 \%$ & $100 \%$ \\
\hline Gemeinsame Technologieinitiativen & $26 \%$ & $16 \%$ & $5 \%$ & $8 \%$ & $45 \%$ & $29 \%$ \\
\hline ARTEMIS & $52 \%$ & $17 \%$ & $19 \%$ & $23 \%$ & $39 \%$ & $31 \%$ \\
\hline ENIAC & $13 \%$ & $15 \%$ & $3 \%$ & $7 \%$ & $47 \%$ & $22 \%$ \\
\hline ECSEL & $24 \%$ & $16 \%$ & $3 \%$ & $5 \%$ & $55 \%$ & $34 \%$ \\
\hline Demographischer Wandel & $89 \%$ & $86 \%$ & $85 \%$ & $90 \%$ & $59 \%$ & $37 \%$ \\
\hline AAL & $90 \%$ & $83 \%$ & $89 \%$ & $93 \%$ & $59 \%$ & $40 \%$ \\
\hline benefit & $88 \%$ & $87 \%$ & $80 \%$ & $87 \%$ & $58 \%$ & $35 \%$ \\
\hline benefit Dienstleistungen & $100 \%$ & $100 \%$ & $100 \%$ & $100 \%$ & $100 \%$ & - \\
\hline Gesamt & $64 \%$ & $56 \%$ & $16 \%$ & $26 \%$ & $59 \%$ & $31 \%$ \\
\hline
\end{tabular}

" bis zum 01.08.2017 entschiedene Projekte; Quelle: FFG, eigene Berechnung inspire research

Während die durchschnittlichen genehmigten Gesamtkosten bei KMU-Anträgen in der nationalen Förderung von 94 Tsd. Euro in den Programmlinien von FIT-IT auf 121 Tsd. Euro in IKT der Zukunft zugenommen haben, verringerten sich die genehmigten Gesamtkosten bei Großunternehmen von durchschnittlich 329 Tsd. Euro im Impulsprogramm FIT-IT auf 196 Tsd. Euro in der Themeninitiative IKT der Zukunft.

Die durchschnittlichen Förderquoten für Unternehmen haben sich in der nationalen kooperativen FuE-Projektförderung zwischen dem Impulsprogramm FIT-IT und IKT der Zukunft kaum verändert bzw. sind für die Gruppe der KMU leicht angestiegen. Einen deutlichen Rückgang der Förderquote von $8 \%$-Punkten bei KMU und 10\%-Punkten bei Großunternehmen gab es in Projekten der Gemeinsamen Technologieinitiativen. Die durchschnittliche Förderquote in Projekten zum Demographischen Wandel lag bisher in der Themeninitiative "IKT der Zukunft" für KMU auf ähnlichem Niveau wie in den Projekten während der Laufzeit des Impulsprogramms FIT-IT, für Großunternehmen ist sie um etwa $5 \%$-Punkte zurückgegangen.

Durch den gestiegenen Anteil des Projekt- und Fördervolumens in Vorhaben der Gemeinsamen Technologieinitiativen ist in der Themeninitiative IKT der Zukunft im Vergleich zum Impulsprogramm FIT-IT der KMU-Kostenanteil (von $23 \%$ auf $16 \%$ ) und der KMU-Barwertanteil (30\% auf $26 \%$ ) zurückgegangen.

Die folgenden beiden Abbildungen illustrieren diese Entwicklungen wieder graphisch. Abbildung 6 zeigt den Umfang der bewilligten Barwerte für Unternehmen über die Projektlaufzeiten aggregiert. Abbildung 7 zeigt die Verteilung der Förderbarwerte, die Unternehmen genehmigt, zwischen KMU und großen Unternehmen. 
Abbildung 6 Summe der Barwerte der Förderung für Unternehmen in den BMVIT IKT-Programmen verteilt auf die Projektlaufzeit nach Unternehmensgröße (Mio. Euro)

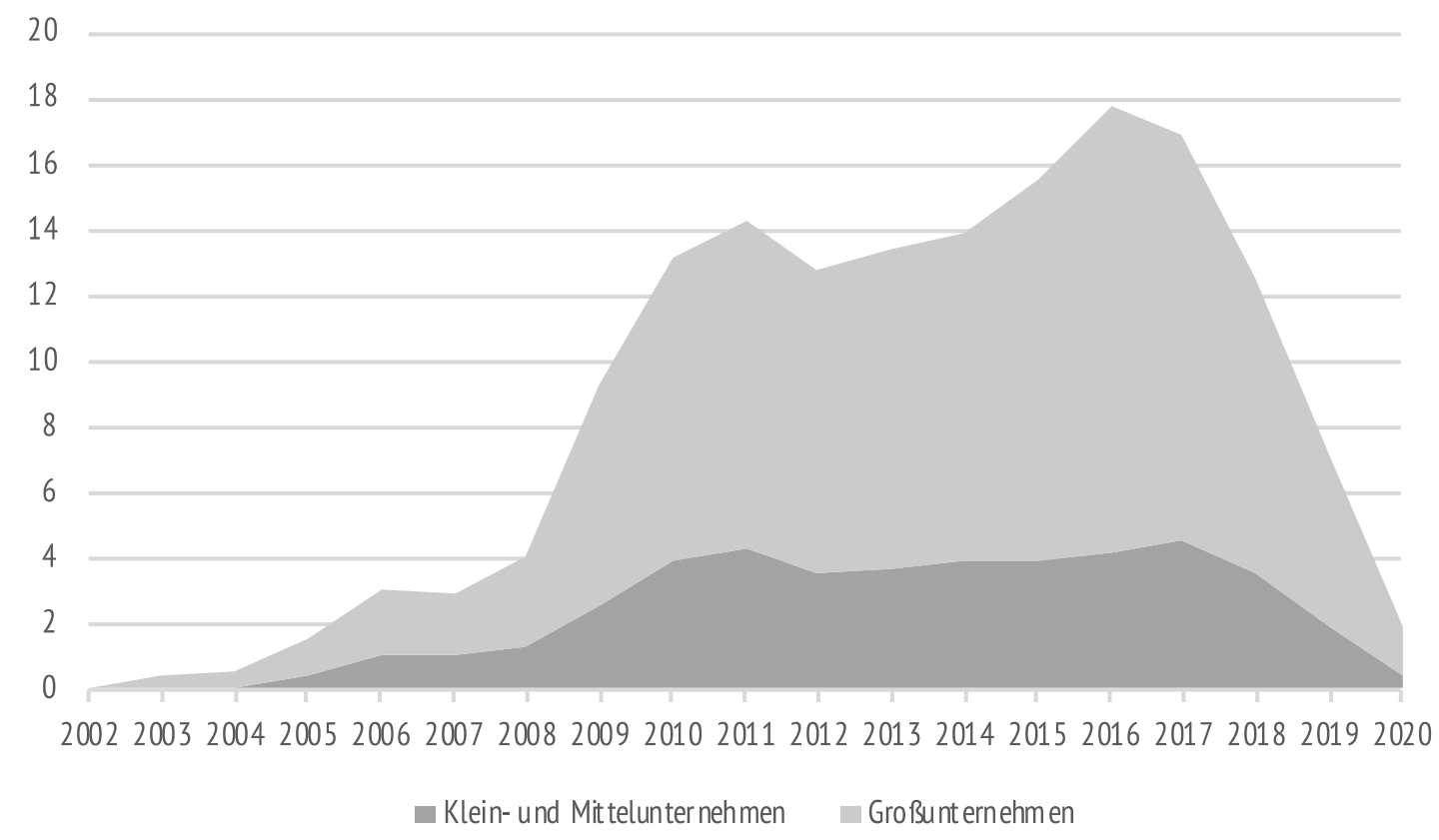

Quelle: FFG, Auswertung und Darstellung inspire research

Seit dem Jahr 2010 verläuft die Förderung von KMU-Projekten nominal fast in einem konstanten Umfang, während die Förderung von Projekten von Großunternehmen auch in den Folgejahren deutlich zugenommen hat. Seit 2007 nimmt der KMU-Fördermittelanteil an den insgesamt den Unternehmen zur Verfügung gestellten Mitteln tendenziell ab.

Abbildung 7 Anteile an der Summe der Barwerte der Förderung für Unternehmen in den BMVIT IKTProgrammen verteilt auf die Projektlaufzeit nach Unternehmensgröße (in \%)

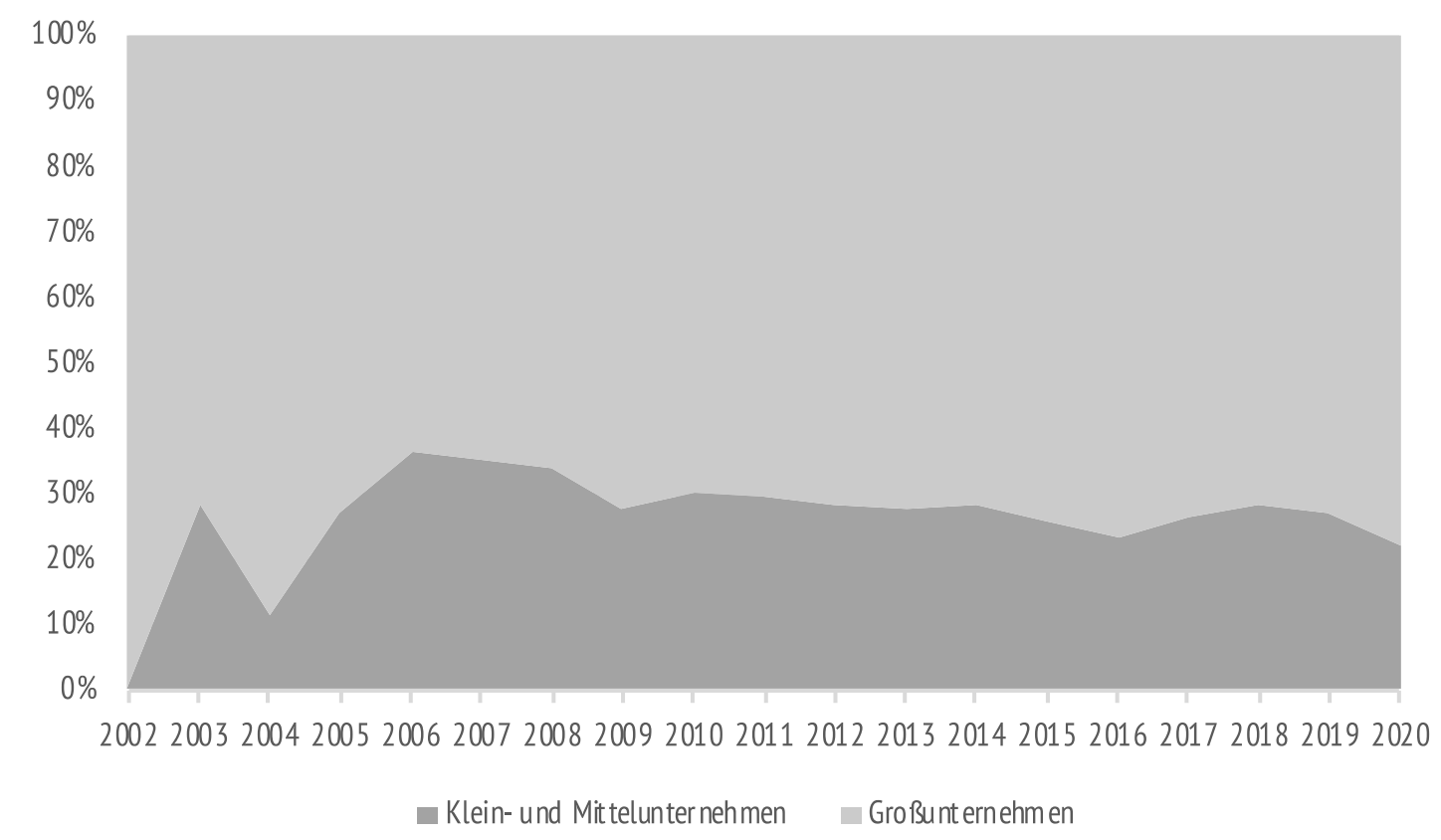

Quelle: FFG, Auswertung und Darstellung inspire research 


\subsection{Beteiligung erstmaliger Antragsteller/innen in FFG-Programmen}

In welchem Umfang ist es der BMVIT-Projektförderung gelungen, neue Akteure für die Mitarbeit in den FuE-Projekten anzusprechen?

Tabelle 15 Impulsprogramm FIT-IT 2002-2012: Anteil der Einrichtungen, die erstmals einen FFG-Antrag stellten

\begin{tabular}{|c|c|c|c|c|}
\hline Programm / Programmlinie & $\begin{array}{c}\text { Alle Antrag- } \\
\text { steller }\end{array}$ & $\begin{array}{c}\text { Genehmigte } \\
\text { Antragst. }\end{array}$ & $\begin{array}{c}\text { Nur Unter- } \\
\text { nehmen }\end{array}$ & $\begin{array}{c}\text { Genehm. Un- } \\
\text { ternehmen }\end{array}$ \\
\hline Impulsprogramm FIT-IT & $\mathbf{1 6 \%}$ & $\mathbf{1 6 \%}$ & $\mathbf{2 0 \%}$ & $\mathbf{1 7 \%}$ \\
\hline Embedded Systems & $16 \%$ & $15 \%$ & $15 \%$ & $9 \%$ \\
\hline Semantic Systems & $22 \%$ & $23 \%$ & $29 \%$ & $29 \%$ \\
\hline Systems on Chip & $4 \%$ & $6 \%$ & $2 \%$ & $4 \%$ \\
\hline Trust in IST & $11 \%$ & $5 \%$ & $17 \%$ & $9 \%$ \\
\hline Visual Computing & $16 \%$ & $18 \%$ & $22 \%$ & $21 \%$ \\
\hline Initiativen \& Joint Call & $14 \%$ & $12 \%$ & $21 \%$ & $15 \%$ \\
\hline Gemeinsame Technologieinitiativen & $\mathbf{2 \%}$ & $\mathbf{2} \%$ & $\mathbf{3} \%$ & $\mathbf{3} \%$ \\
\hline ARTEMIS & $2 \%$ & - & $1 \%$ & - \\
\hline ENIAC & $3 \%$ & $4 \%$ & $5 \%$ & $6 \%$ \\
\hline Demographischer Wandel & $\mathbf{2 1 \%}$ & $\mathbf{2 1 \%}$ & $\mathbf{2 9} \%$ & $\mathbf{3 0 \%}$ \\
\hline AAL & $16 \%$ & $15 \%$ & $27 \%$ & $32 \%$ \\
\hline benefit & $23 \%$ & $23 \%$ & $31 \%$ & $29 \%$ \\
\hline Gesamt & $\mathbf{1 6 \%}$ & $\mathbf{1 6} \%$ & $\mathbf{2 0} \%$ & $\mathbf{1 8 \%}$ \\
\hline Quelle: FFG, eigene Berechnung & & & \\
\hline
\end{tabular}

Quelle: FFG, eigene Berechnung inspire research

Die Auswertung der FFG-Antrags- und Förderdaten zeigt, dass es insbesondere dem Thema Demographischer Wandel gut gelingt, neue Akteure für die FuE-Projekte zu gewinnen. Vor allem in den ersten Jahren der AAL- und benefit-Förderung lag der Anteil der erstmals einreichenden Einrichtungen sehr hoch. Während der Laufzeit des Impulsprogramms FIT-IT waren $21 \%$ der Antragsteller in Projekten zum Demographischen Wandels erstmalige Einreicher, in der Gruppe der Unternehmen sogar 29 \%. Keine Rolle spielen Neueinreicher hingegen in den Gemeinsamen Technologieinitiativen.

Tabelle 16 BMVIT Themeninitiative IKT der Zukunft 2012-2016*: Anteil der Einrichtungen, die erstmals einen FFG-Antrag stellten

\begin{tabular}{|c|c|c|c|c|}
\hline Themeninitiative IKT der Zukunft & $\begin{array}{c}\text { Alle Antrag- } \\
\text { steller }\end{array}$ & $\begin{array}{c}\text { Genehmigte } \\
\text { Antragst. }\end{array}$ & $\begin{array}{c}\text { Nur Unter- } \\
\text { nehmen }\end{array}$ & $\begin{array}{c}\text { Genehm. Un- } \\
\text { ternehmen }\end{array}$ \\
\hline IKT der Zukunft & $\mathbf{8} \%$ & $\mathbf{6 \%}$ & $\mathbf{1 3 \%}$ & $\mathbf{8 \%}$ \\
\hline IKT der Zukunft & $8 \%$ & $5 \%$ & $12 \%$ & $7 \%$ \\
\hline IKT der Zukunft Dienstleistungen & $12 \%$ & $14 \%$ & $25 \%$ & $33 \%$ \\
\hline IKT der Zukunft Professur & - & - & - & - \\
\hline Gemeinsame Technologieinitiativen & $\mathbf{1 \%}$ & - & $\mathbf{2 \%}$ & - \\
\hline ARTEMIS & - & - & - & - \\
\hline ENIAC & - & - & - & - \\
\hline ECSEL & $2 \%$ & - & $3 \%$ & - \\
\hline Demographischer Wandel & $\mathbf{1 2} \%$ & $\mathbf{1 4 \%}$ & $\mathbf{1 7 \%}$ & $\mathbf{1 9 \%}$ \\
\hline AAL & $5 \%$ & $5 \%$ & $11 \%$ & $8 \%$ \\
\hline benefit & $15 \%$ & $19 \%$ & $21 \%$ & $25 \%$ \\
\hline benefit Dienstleistungen & $47 \%$ & $25 \%$ & - & - \\
\hline Gesamt & $\mathbf{8} \%$ & $\mathbf{8 \%}$ & $\mathbf{1 2} \%$ & $\mathbf{1 0 \%}$ \\
\hline
\end{tabular}

"bis zum 01.08.2017 entschiedene Projekte; Quelle: FFG, eigene Berechnung inspire research 
In der Themeninitiative "IKT der Zukunft" ist der Anteil der neueinreichenden Einrichtungen im Vergleich zum Impulsprogramm FIT-IT deutlich zurückgegangen. Dies ist ein Effekt von Wiedereinreichungen in den BMVIT IKT-Programmen selbst - 166 der bisher 514 Ersteinreicher (32\%) seit Start des Impulsprogramms FIT-IT haben bis 2016 zumindest einen weiteren Antrag in den BMVIT IKT-Programmen gestellt. Eine Rolle dürfte aber auch das seit dem Jahr 2002 doch sehr deutlich erweiterte Förderangebot an anderen FuE-Programmen spielen.

In wie weit gelang bzw. gelingt es den in den BMVIT IKT-Programmen nicht-geförderten Unternehmen, Förderungen aus anderen FFG-Quellen zu erhalten? Eine Auswertung der FFGAntragsdaten zeigt, dass insgesamt rund die Hälfte der nicht in den BMVIT IKT-Programmen geförderten Unternehmen eine Förderung aus dem FFG-Basisprogramm erhielten. Am Programm beteiligte, aber nicht geförderte Großunternehmen wurden dabei bereits meist vor dem Jahr 2006 erstmals im FFG-Basisprogramm gefördert. Die nicht in den IKT-Programmen geförderten KMU erhielten zu einem größeren Anteil erstmals zwischen 2007 und 2016 eine Förderung im FFG-Basisprogramm.

Nur $16 \%$ der im Zeitraum 2002 bis 2016 Antrag stellenden Großunternehmen, aber $29 \%$ der KMU erhielten weder in den BMVIT IKT-Programmen noch im FFG Basisprogramm eine Förderung. Eine Auswertung nach Programmen zeigt, dass vor allem Unternehmen in den Ausschreibungen zum Demographischen Wandel nach einer Ablehnung auch weniger häufig im FFG-Basisprogramm gefördert werden.

Abbildung 8 Anzahl der geförderten und nicht geförderten antragstellenden Unternehmen in den BMVIT IKT-Programmen 2002-2016 sowie im FFG-Basisprogramm (BP) nach Unternehmensgröße

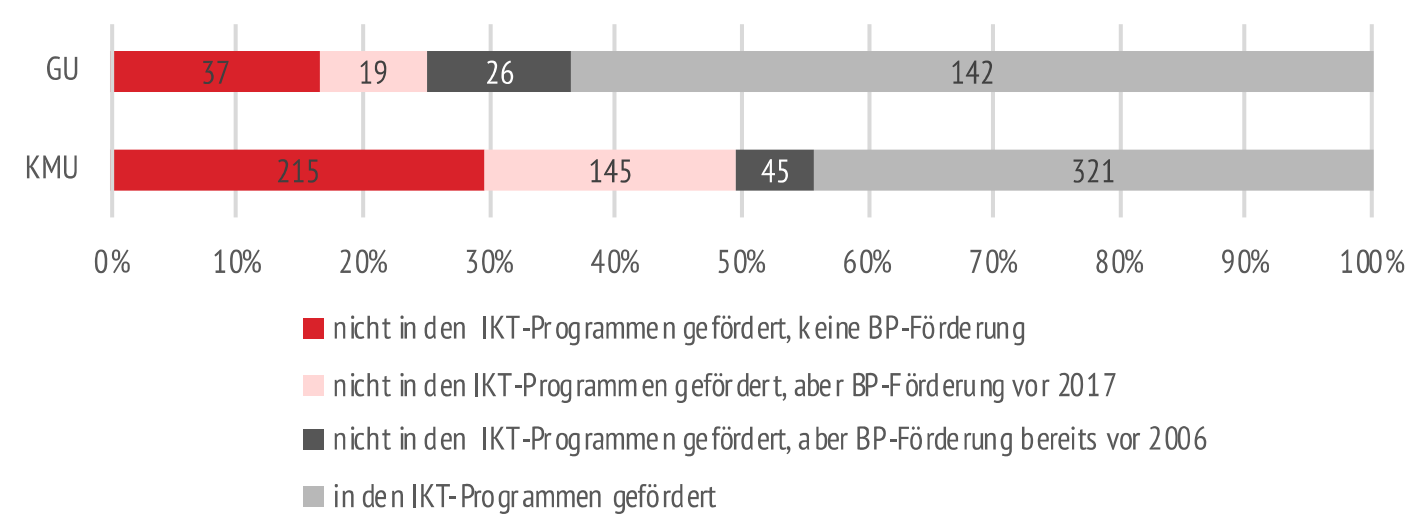

Quelle: FFG, Auswertung und Darstellung inspire research

Während $55 \%$ der nicht geförderten Unternehmen, die sich an den nationalen Programmen FIT-IT / IKT der Zukunft bzw. an den Gemeinsamen Technologieinitiativen beteiligt hatten, schließlich eine Förderung im FFG-Basisprogramm erhielten, liegt dieser Anteil in den beiden Programmen zum Demographischen Wandel (benefit, AAL) bei nur 30 \% (siehe Abbildung 9 , folgende Seite). 
Abbildung 9 Nicht-geförderte Antrag stellende Unternehmen in den IKT-Programmen 2002-2016 nach Unternehmensgröße: Förderung im Basisprogramm (BP)

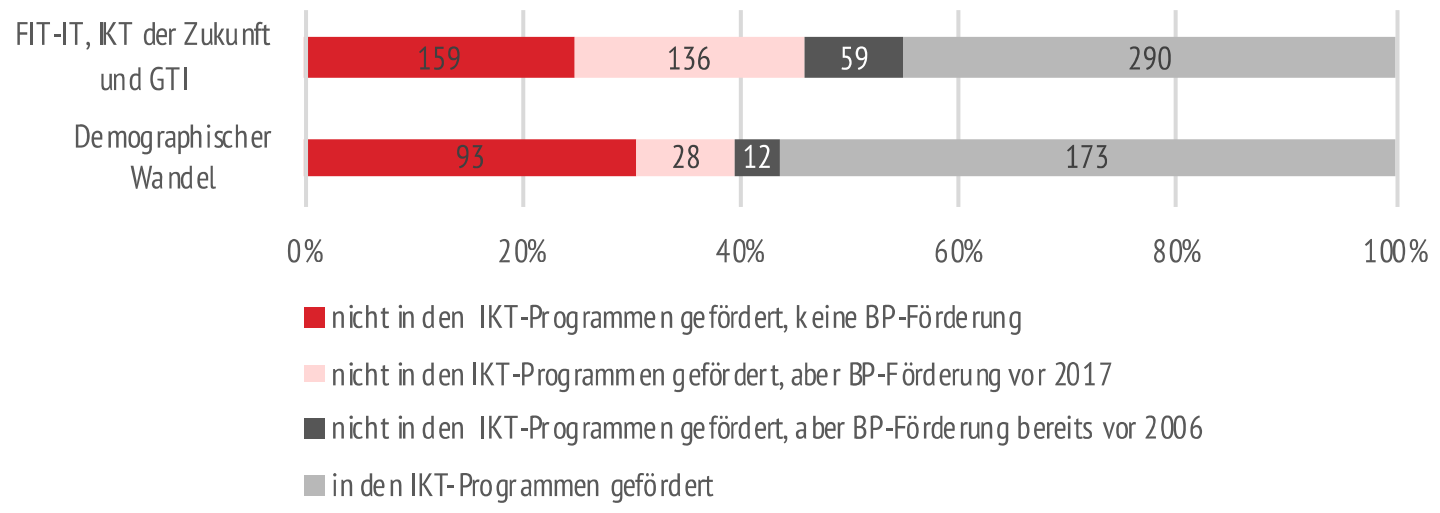

Quelle: FFG, Auswertung und Darstellung inspire research

Für alle Unternehmensgrößenklassen liegt der öffentliche Finanzierungsanteil (ohne Berücksichtigung der Forschungsprämie) an den internen FuE-Ausgaben in der Erhebung 2013 bei Unternehmen, die in den Jahren 2010 bis 2013 eine Förderung aus den BMVIT-IKT Programmen erhielten, sehr deutlich über den Niveaus in der Gruppe der antragstellenden, nicht geförderten Unternehmen. Dass sich aggregiert für alle Unternehmen bzw. für produzierende Unternehmen keine Unterschiede zeigen, hat mit den unterschiedlichen Anteilen an Klein-, Mittel- und Großunternehmen in den beiden Gruppen zu tun (d. h. deutlich höherer Anteil von Großunternehmen in der Gruppe der geförderten Unternehmen in der FuE-Erhebung 2013).

Abbildung 10 Anteil der direkten öffentlichen Förderung (d. h. ohne Finanzierungsbeitrag der Forschungsprämie) an den internen FuE-Ausgaben der geförderten und der Antrag stellenden, nicht-geförderte Unternehmen in den BMVIT IKT-Programmen 2010 bis 2013, die in der amtlichen F\&E-Statistik 2013 erfasst sind, nach Wirtschaftssektoren und Unternehmensgrößenklassen (Mittelwerte)

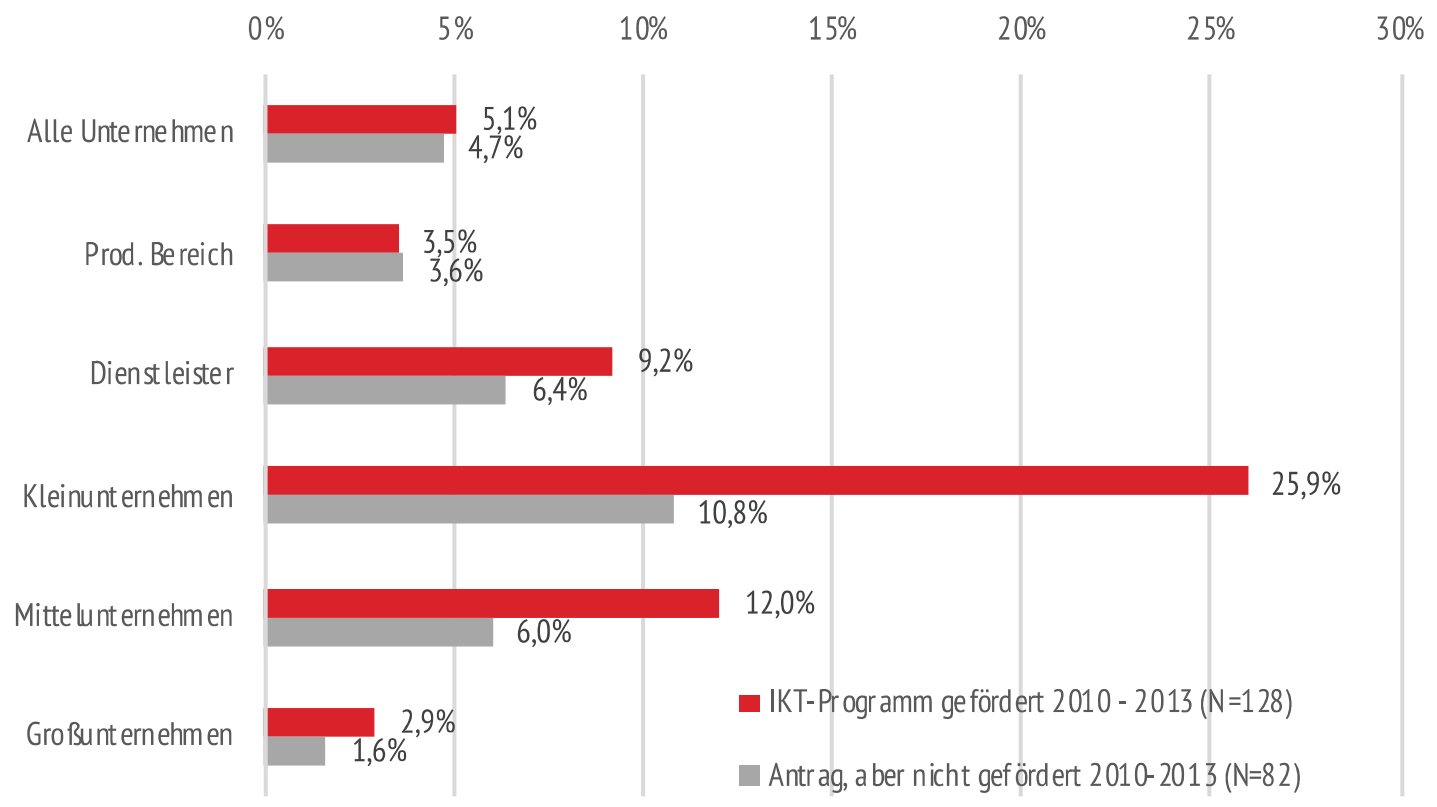

Quelle: FFG, Auswertung und Darstellung inspire research

Außerdem zeigt die Analyse der Daten der FuE-Statistik von Statistik Austria, dass es sich bei den einreichenden, nicht geförderten Unternehmen insgesamt um deutlich forschungsschwächere Einrichtungen handelt. Gemäß FuE-Erhebung 2013 stand den geförderten Unternehmen im Jahr 2013 mehr als dreimal so viel Personal für Forschung und Entwicklung (in Vollzeitäquivalenten) zur Verfügung als der Gruppe der nicht geförderten Unternehmen. 
Abbildung 11 Personal für Forschung und Entwicklung (VZÄ) der geförderten und der antragstellenden nicht-geförderte Unternehmen in den BMVIT IKT-Programmen 2010 bis 2013, die in der amtlichen F\&E-Statistik 2013 erfasst sind, nach Wirtschaftssektoren und Unternehmensgrößenklassen (Mittelwerte)

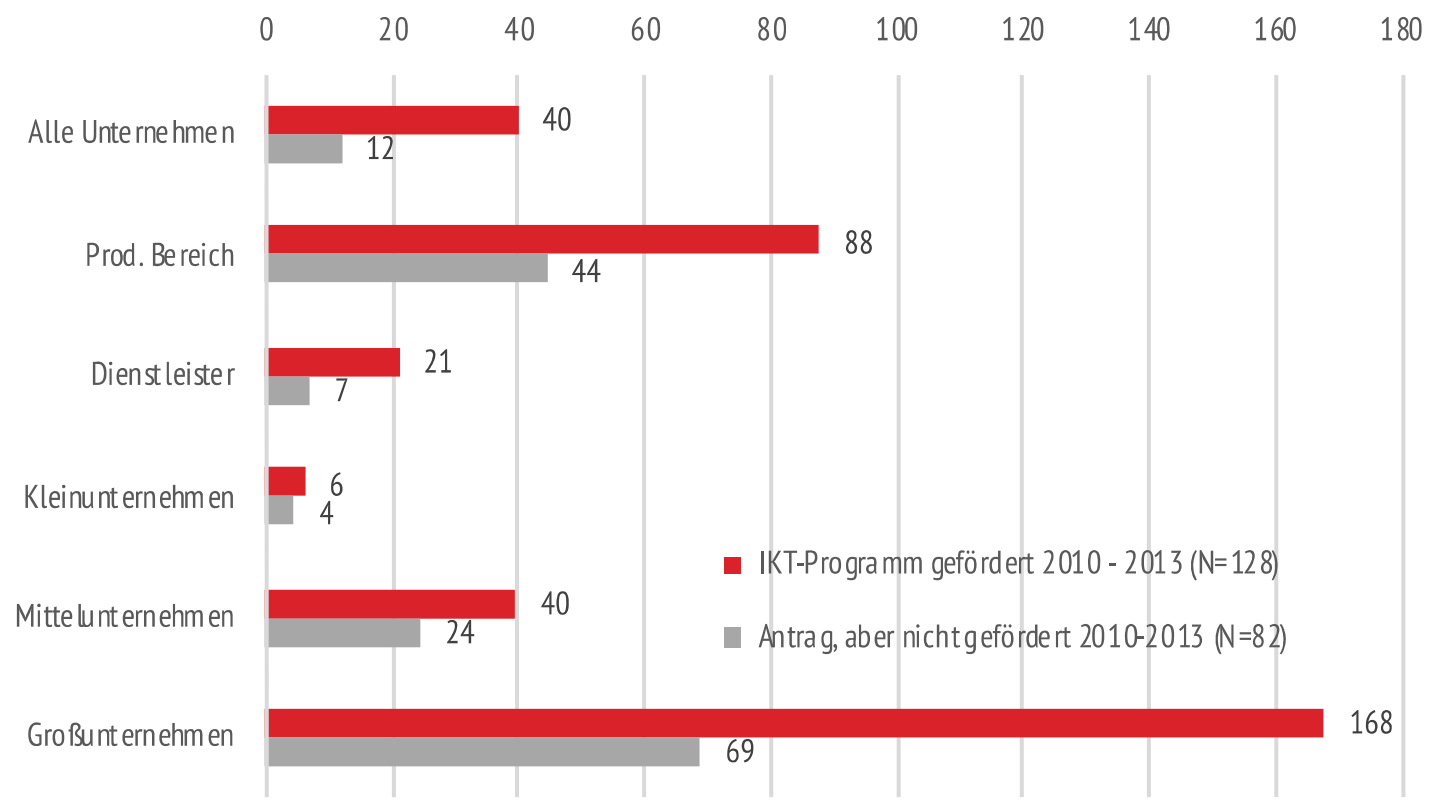

Quelle: FFG, Statistik Austria, Auswertung und Darstellung inspire research

\subsection{Beteiligung von Frauen als technische Ansprechpersonen}

Wie hat sich die Beteiligung von Frauen an den Anträgen und Projekten der BMVIT IKT-Programme seit 2002 entwickelt? Zeigen sich hier Unterschiede zwischen verschiedenen Programmen bzw. Förderthemen?

Ziehen wir als einen groben Indikator für die Entwicklung der Beteiligung von Frauen in den BMVIT IKT-Programmen den Frauenanteil unter den in der FFG-Datenbank erfassten technischen Ansprechpersonen (die in vielen Fällen auch die operativen Projektleiter/innen der Projekte sind) heran, so zeigt sich, dass während der Laufzeit des Impulsprogramms FIT-IT ein substanzieller Frauenanteil nur in den Projekten zu Themen des Demographischen Wandels gegeben war. Fast ein Viertel der technischen Ansprechpersonen in Anträgen zum Demographischen Wandel waren Frauen. In Projekten der Gemeinsamen Technologieinitiativen ARTEMIS und ENIAC war der Frauenanteil unter den technischen Ansprechpersonen hingegen besonders gering. Aber auch in den verschiedenen Programmlinien von FIT-IT lag der Frauenanteil unter den technischen Ansprechpersonen bei maximal $10 \%$.

Der Anteil der Frauen unter den technischen Ansprechpersonen unterscheidet sich zwischen eingereichten und genehmigten Projektanträgen nur unwesentlich. Der Vergleich zwischen Teilnehmergruppen zeigt, dass der Frauenanteil unter den technischen Ansprechpersonen bei Unternehmen mit rund $8 \%$ besonders gering war. Aber auch an Hochschulen und außeruniversitären Forschungseinrichtungen lag der Frauenanteil bei nur $10 \%$ bzw. $11 \%$. Den höchsten Anteil an Frauen unter den technischen Ansprechpersonen gab es mit fast einem Viertel (22\%) in der Gruppe der sonstigen Antragsteller.

Die FFG-Antragsdaten für die bisherigen Ausschreibungen in der Themeninitiative "IKT der Zukunft" zeigen in allen Förderthemen einen leicht steigenden Frauenanteil unter den technischen Ansprechpersonen. In den nationalen FuE-Projekten von IKT der Zukunft liegt der 
Frauenanteil unter den technischen Ansprechpersonen bei genehmigten Anträgen mit $13 \%$ deutlich über dem Niveau des Impulsprogramms FIT-IT (7\%).

Tabelle 17 Impulsprogramm FIT-IT: Anteil der Frauen unter den technischen Ansprechpersonen in den Anträgen nach Programmen / Programmlinien und Teilnehmergruppen 2002-2012

\begin{tabular}{|c|c|c|c|c|c|}
\hline Programm / Programmlinie & $\begin{array}{c}\text { Hoch- } \\
\text { schulen }\end{array}$ & $\begin{array}{c}\text { Forsch.- } \\
\text { einricht. }\end{array}$ & $\begin{array}{c}\text { Unter- } \\
\text { nehmen }\end{array}$ & Sonstige & Gesamt \\
\hline Impulsprogramm FIT-IT & $\mathbf{7 \%}$ & $\mathbf{8} \%$ & $\mathbf{6 \%}$ & $\mathbf{2 0 \%}$ & $\mathbf{7 \%}$ \\
\hline Embedded Systems & $3 \%$ & $2 \%$ & $5 \%$ & $14 \%$ & $4 \%$ \\
\hline Semantic Systems & $11 \%$ & $15 \%$ & $6 \%$ & $14 \%$ & $10 \%$ \\
\hline Systems on Chip & $2 \%$ & $0 \%$ & $0 \%$ & - & $1 \%$ \\
\hline Trust in ITS & $6 \%$ & $0 \%$ & $5 \%$ & $0 \%$ & $4 \%$ \\
\hline Visual Computing & $8 \%$ & $4 \%$ & $6 \%$ & $50 \%$ & $7 \%$ \\
\hline Initiativen \& Joint Call & $6 \%$ & $13 \%$ & $9 \%$ & $27 \%$ & $10 \%$ \\
\hline Gemeinsame Technologieinitiativen & $\mathbf{8} \%$ & $\mathbf{3} \%$ & $\mathbf{3} \%$ & $\mathbf{0} \%$ & $\mathbf{4} \%$ \\
\hline ARTEMIS & $17 \%$ & $4 \%$ & $3 \%$ & $0 \%$ & $5 \%$ \\
\hline ENIAC & $4 \%$ & $0 \%$ & $3 \%$ & - & $3 \%$ \\
\hline Demographischer Wandel & $\mathbf{3 2} \%$ & $\mathbf{2 0} \%$ & $\mathbf{1 9} \%$ & $\mathbf{2 5 \%}$ & $\mathbf{2 3} \%$ \\
\hline AAL & $39 \%$ & $18 \%$ & $25 \%$ & $35 \%$ & $27 \%$ \\
\hline benefit & $29 \%$ & $22 \%$ & $17 \%$ & $21 \%$ & $21 \%$ \\
\hline Gesamt & $\mathbf{1 0} \%$ & $\mathbf{1 1} \%$ & $\mathbf{8} \%$ & $\mathbf{2 2} \%$ & $\mathbf{1 0 \%}$ \\
\hline
\end{tabular}

Quelle: FFG, eigene Berechnung inspire research

Selbst in Projektanträgen der Gemeinsamen Technologieinitiative hat sich der Frauenanteil unter den technischen Ansprechpersonen - wenn auch von einem sehr geringen Niveau ausgehend - merkbar erhöht. Geringe Anstiege zeigen sich weiterhin in den Projekten zum Demographischen Wandel, wobei hier insbesondere der Frauenanteil unter den technischen Ansprechpersonen im nationalen Programm benefit zugenommen hat.

Tabelle 18 Themeninitiative "IKT der Zukunft": Anteil der Frauen unter den technischen Ansprechpersonen in den Anträgen nach Förderthemen und Teilnehmergruppen 2012-2016*

\begin{tabular}{|c|c|c|c|c|c|}
\hline Förderthemen & $\begin{array}{c}\text { Hoch- } \\
\text { schulen }\end{array}$ & $\begin{array}{c}\text { Forsch.- } \\
\text { einricht. }\end{array}$ & $\begin{array}{c}\text { Unter- } \\
\text { nehmen }\end{array}$ & Sonstige & Gesamt \\
\hline IKT der Zukunft & $\mathbf{1 2 \%}$ & $\mathbf{1 3} \%$ & $\mathbf{6 \%}$ & $\mathbf{1 6 \%}$ & $\mathbf{9 \%}$ \\
\hline IKT der Zukunft & $11 \%$ & $12 \%$ & $6 \%$ & $18 \%$ & $9 \%$ \\
\hline IKT der Zukunft Dienstleistungen & $10 \%$ & $24 \%$ & $0 \%$ & $0 \%$ & $12 \%$ \\
\hline IKT der Zukunft Professur & $33 \%$ & - & - & - & $33 \%$ \\
\hline Gemeinsame Technologieinitiativen & $\mathbf{5 \%}$ & $\mathbf{9 \%}$ & $\mathbf{8 \%}$ & - & $\mathbf{8 \%}$ \\
\hline ARTEMIS & $0 \%$ & $25 \%$ & $4 \%$ & - & $9 \%$ \\
\hline ENIAC & $0 \%$ & $9 \%$ & $7 \%$ & - & $6 \%$ \\
\hline ECSEL & $7 \%$ & $5 \%$ & $9 \%$ & - & $8 \%$ \\
\hline Demographischer Wandel & $\mathbf{4 5 \%}$ & $\mathbf{2 3} \%$ & $\mathbf{1 4 \%}$ & $\mathbf{3 6 \%}$ & $\mathbf{2 6 \%}$ \\
\hline AAL & $37 \%$ & $14 \%$ & $15 \%$ & $49 \%$ & $25 \%$ \\
\hline benefit & $50 \%$ & $32 \%$ & $13 \%$ & $25 \%$ & $27 \%$ \\
\hline benefit Dienstleistungen & $50 \%$ & $33 \%$ & $33 \%$ & $43 \%$ & $41 \%$ \\
\hline Gesamt & $\mathbf{2 3 \%}$ & $\mathbf{1 6 \%}$ & $\mathbf{9} \%$ & $\mathbf{3 3 \%}$ & $\mathbf{1 6 \%}$ \\
\hline
\end{tabular}

"bis zum 01.08.2017 entschiedene Anträge; Quelle: FFG, eigene Berechnung inspire research

Die Auswertungen der FFG-Daten deuten damit auf eine über die Jahre steigende Beteiligung von Frauen in operativ tragenden Rollen der Projekte in den BMVIT IKT-Programmen hin, wobei jedoch nach wie vor Frauen - vor allem in den Vorhaben der Unternehmen - stark unterrepräsentiert sind. 


\subsection{Wissenschaftliche Leistungen der Antragsteller und Projektauswahl}

Welche Projektanträge wurden im Impulsprogramm FIT-IT für die Förderung bevorzugt ausgewählt? Waren es eher bereits durch Publikationen gut ausgewiesene Wissenschaftler/innen oder eher noch weniger publikationsaktive Wissenschaftler/innen, deren Projekte gefördert wurden? Lassen sich Unterschiede zwischen den drei Programmen ausmachen?

Um diese Fragen zu beantworten, haben wir die in der Publikationsdatenbank Scopus erfassten Publikationen der technischen Ansprechpersonen bei den wissenschaftlichen Einrichtungen (d. h. Hochschulen und außeruniversitäre Forschungseinrichtungen), die Anträge während der Laufzeit des Impulsprogramms FIT-IT gestellt haben, ausgewertet. Als Indikatoren für die wissenschaftlichen Leistungen der Antragsteller/innen ziehen wir dabei den aktuellen h-Index ${ }^{2}$ (Stand: Oktober 2017) der Wissenschaftler/innen sowie die durchschnittliche Anzahl der Zitationen ihrer Publikationen (Stand: Oktober 2017) heran.

Wir sind uns bewusst, dass mit diesem Ansatz ein methodisches Problem verbunden ist. Da wir den h-Index bzw. die wissenschaftliche Rezeption der bereits zum Zeitpunkt der Antragstellung veröffentlichten wissenschaftlichen Publikationen in Scopus mit vertretbarem Aufwand nicht direkt erheben können, spiegelt die Auswertung mit den aktuellen Werten streng genommen auch den weiteren wissenschaftlichen Karriereverlauf der Antragsteller/innen wider und nicht nur jenen bis zum Zeitpunkt der Projekteinreichung. Nehmen wir jedoch an, dass sich die wissenschaftliche Publikationstätigkeit sowohl in der Gruppe der geförderten als auch der nicht-geförderten Wissenschaftler/innen stetig und in vergleichbarem Ausmaß weiterentwickelt, können wir die aktuellen Werte zumindest im Verhältnis zueinander als Schätzer für die Situation bei der Entscheidung über die Projekte heranziehen.

Insgesamt sind die in der FFG-Datenbank angeführten technischen Ansprechpersonen der wissenschaftlichen Einrichtungen bibliometrisch gut erfasst. Für in Summe 377 von 390 (97\%) technischen Ansprechpersonen aus Hochschulen und außeruniversitären Forschungsreinrichtungen mit genehmigten Anträgen sowie für 240 von 259 (93 \%) technische Ansprechpersonen mit nur abgelehnten Anträgen in den Jahren 2002 bis 2012 können in der Scopus-Datenbank Einträge über wissenschaftliche Publikationen gefunden werden.

Abbildung 12 Technische Ansprechpersonen der wissenschaftlichen Partner (Hochschulen, außeruniversitäre Forschungseinrichtungen) in FIT-IT Anträgen mit und ohne Scopus-Eintrag

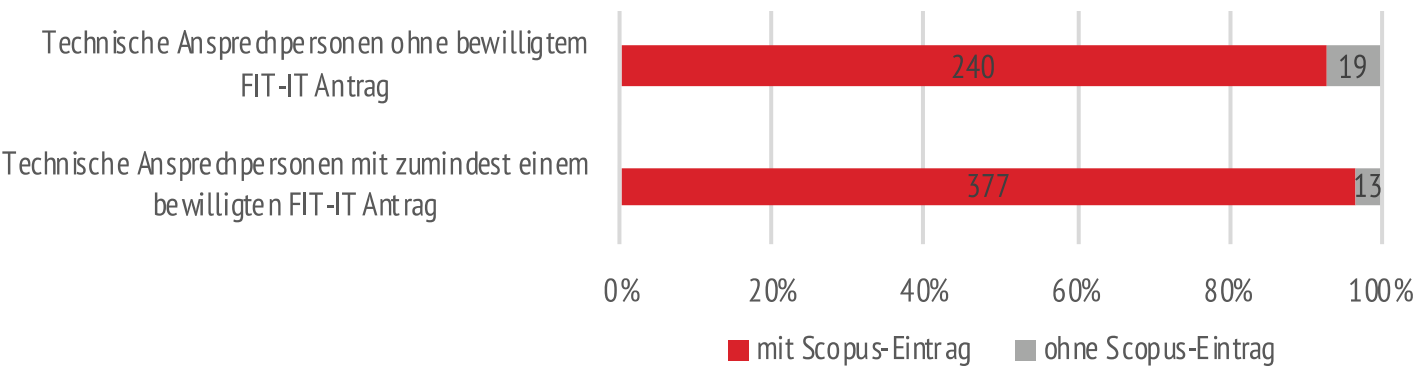

Quelle: FFG, Scopus; Auswertung und Darstellung inspire research

Ziehen wir den h-Index als Indikator für das Ausmaß der wissenschaftlichen Rezeption (und indirekt auch für Seniorität innerhalb eines einzelnen Forschungsfachs) heran, wird deutlich, dass es sich bei den Projekten in den FIT-IT Programmlinien am häufigsten um Projekte gehandelt hat, die auch von wissenschaftlich besonders ausgewiesenen und (bereits) gut rezi-

\footnotetext{
${ }^{2}$ Der h-Index ist die [größtmögliche] Anzahl der Publikationen eines Wissenschaftlers / einer Wissenschaftlerin, die mindestens h-mal zitiert wurden.
} 
pierten Wissenschaftler/innen geleitet bzw. koordiniert wurden. Der durchschnittliche h-Index der technischen Ansprechpersonen geförderter FIT-IT-Projekte beträgt 12,6, während der Durchschnitt des h-Index in der Gruppe der Antragsteller/innen ohne genehmigten FITIT Projekt nur 8,3 beträgt.

Etwas geringer ist der Abstand in den durchschnittlichen h-Indices für die Projekte bzw. Anträge in den Gemeinsamen Technologieinitiativen. Hier beträgt der Mittelwert des h-Index für die technischen Ansprechpersonen geförderter Projekte 10,5, in der Gruppe der Antragsteller/innen ohne genehmigtem Projekt durchschnittlich 7,9.

Vergleichsweise am wenigsten durch bereits rezipierte Publikationen ausgewiesen waren die technischen Ansprechpersonen der wissenschaftlichen Partner in Projekten zum Demographischen Wandel. Für diese Projekte sind auch die Unterschiede zwischen den technischen Ansprechpersonen geförderter Projekte und der technischen Ansprechpersonen mit nur abgelehnten Projekten am geringsten. Wir werten diese Ergebnisse als Indiz dafür, dass die Wissenschaftlicher/innen in Projekten zum Demographischen Wandel zu einem stärkeren Anteil Disziplinen angehören, die einerseits bibliographisch weniger gut erfasst sind und andererseits in diesem Programm die Selektion von Projektanträgen weniger stark von den wissenschaftlichen (Vor-) Leistungen der beteiligten Wissenschaftler/innen abhängig war, als in den beiden anderen (Teil-)Programmen.

Abbildung $13 \mathrm{~h}$-Index der technischen Ansprechpersonen der wissenschaftlichen Partner in FIT-IT Anträgen nach Programmen (Mittelwert)

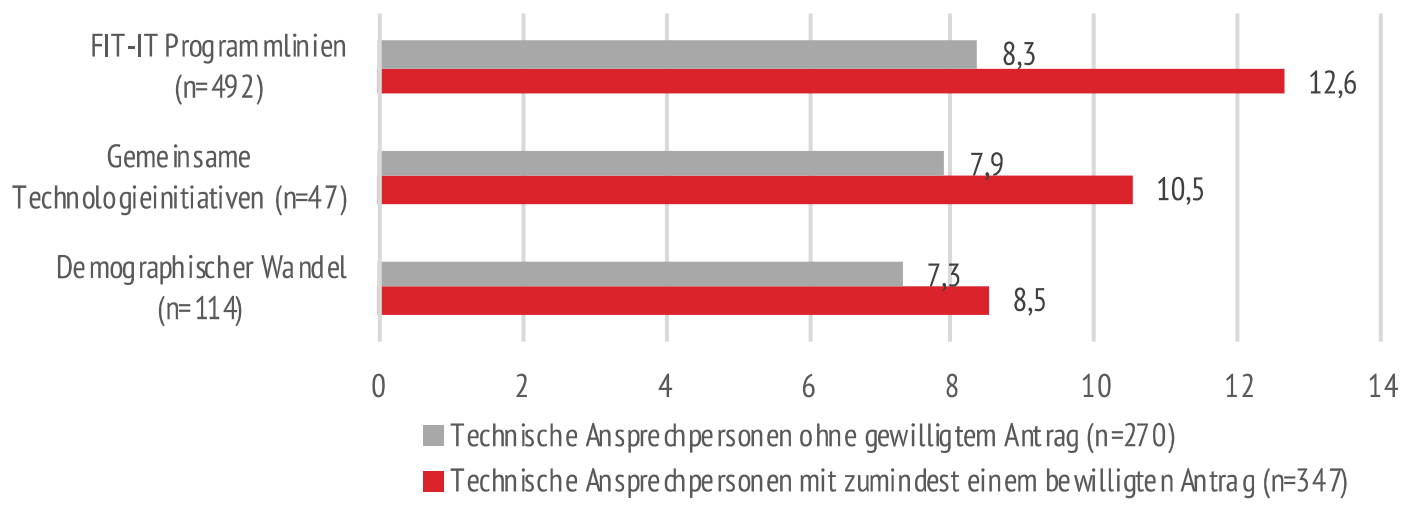

Quelle: FFG, Scopus; Auswertung und Darstellung inspire research

Wenn man die Rezeption der Publikationen der technischen Ansprechpersonen bei den wissenschaftlichen Einrichtungen auf das Jahr des (geplanten) Projektstarts bezieht, sieht man ebenfalls das Ausmaß der Selektionswirkung zwischen (heute) stärker etablierten und (weniger) etablierten Wissenschaftler/innen deutlich.

Die folgende Abbildung zeigt abhängig vom Publikationsjahr die durchschnittliche Anzahl der Zitationen der Publikationen der geförderten und nicht geförderten technischen Ansprechpersonen. Während in den FIT-IT-Programmlinien und in der Gemeinsamen Technologieinitiativen die Unterschiede in den durchschnittlichen Zitationsraten der Publikationen, die vor dem (geplanten) Start des Projekts veröffentlicht wurden, zwischen geförderten und nicht geförderten Wissenschaftler/innen groß sind, fallen sie für die Antragsteller von Projekten zum Demographischen Wandel vergleichsweise gering aus. 
Abbildung 14 Mittelwert der Zitationen der Publikationen der technischen Ansprechpersonen der wissenschaftlichen Partner in FIT-IT Anträgen im Zeitraum zwei Kalenderjahre vor dem Kalenderjahr des (geplanten) Start des Projekts (J-2) bis sechs Kalenderjahre nach dem Kalenderjahr des (geplanten) Start des Projekts $(\mathrm{J}+6)$ nach Programmen

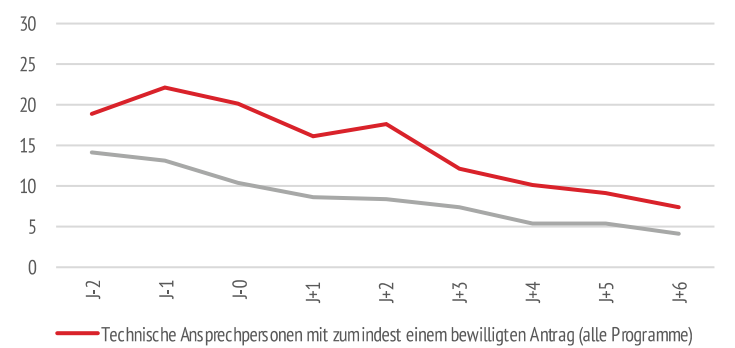

-Technische Ansprechpers onen mit zumindest einem bewilligten Antrag (alle Programme) — Technische Ans prechpers onen ohne gevilligtem Antrag (alle Programme)

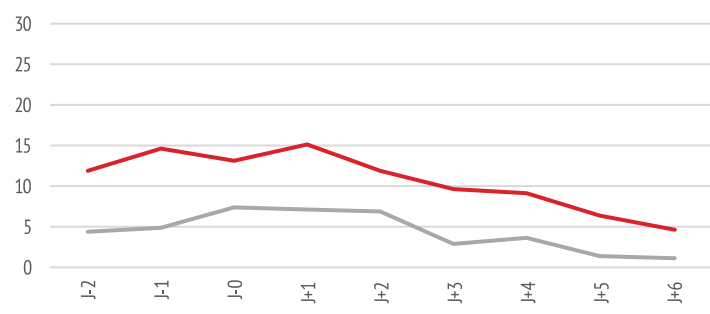

-Techn. Ansprechpers. mit zumind. einem bew. Gemeinsame Technologieinitiative-Antrag — Techn. Ansprechpers. ohne bewilligtem Gemeinsame Technologieinitiative-Antrag
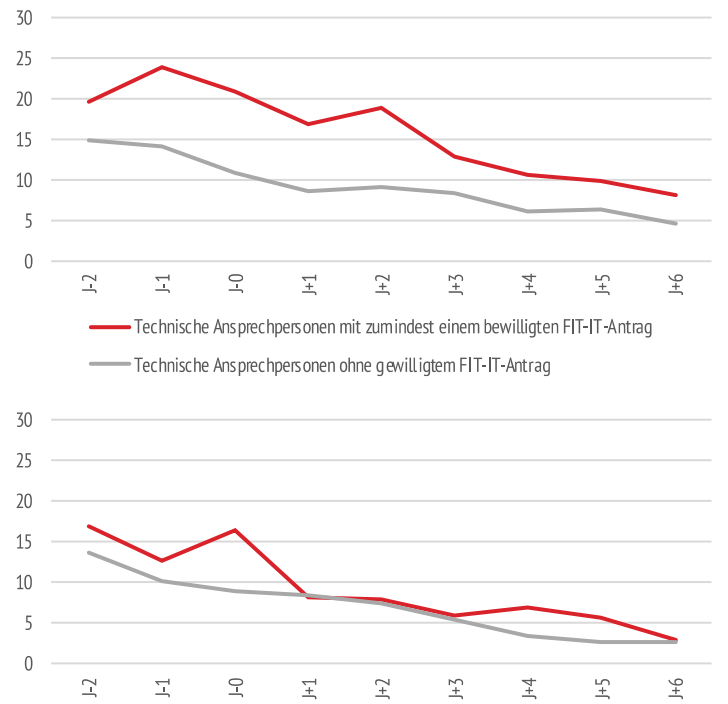

-Techn. Ansprechpersonen mit zumindest einem bew. Demographischen Wandel-Antrag — Techn. Ansprechpersonen ohne bewilligtem Demographischen Wandel-Antrag

Quelle: FFG, Scopus; Auswertung und Darstellung inspire research 


\section{Programmdurchführung}

In diesem Abschnitt präsentieren wir die Ergebnisse der Evaluierung zur Programmumsetzung der Themeninitiative IKT der Zukunft sowie die Hinweise der Förderempfänger über die Zusammenarbeit mit den Projektpartnern und die Erfahrungen mit der administrativen Abwicklung der geförderten Projekte.

\subsection{FuE-Zusammenarbeit und Vorerfahrungen mit den Partnern}

Wie sind die Konsortien in IKT der Zukunft-Projekten zusammengesetzt bzW. welche Rollen und Aufgaben sehen die Projektpartner im Zentrum ihrer Zusammenarbeit? Mit welchem Ziel arbeiten die Partner in den Projekten zusammen?

Mehr als die Hälfte der Unternehmen (55\%) gaben in der Befragung an, die Beteiligung am FuE-Projekt diente der Entwicklung oder Weiterentwicklung eigener Produkte, Verfahren oder Dienstleistungen. Weitere $10 \%$ der Unternehmen sind als FuE-Dienstleister für andere Partner an den Projekten beteiligt. Nicht ganz ein Viertel der Unternehmen in den Projekten sind Anwender von FuE-Ergebnissen anderer Partner im Projekt, im größerem Ausmaß zur Unterstützung der eigenen Entwicklungsaufgaben, im kleineren Ausmaß als reine Pilotanwender von FuE-Lösungen. Nur ein geringer Anteil an den Unternehmen betrieb mit den Projekten FuE, mit der keine unmittelbare wirtschaftliche Verwertung verbunden war.

Abbildung 15 Welche der folgenden Aussagen beschreibt die Aufgabe Ihrer Einrichtung im Projekt am besten? Anzahl der Antworten nach Teilnehmergruppen

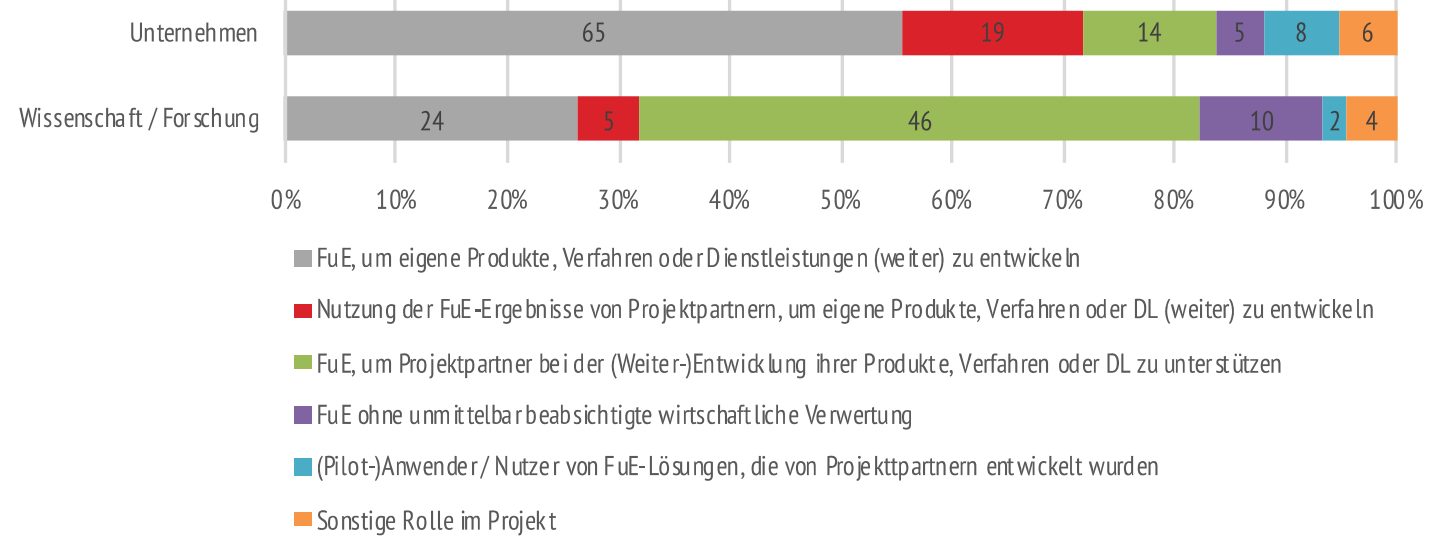

Quelle: Befragung Evaluierung BMVIT-IKT-Programme 2017; inspire research

Auch unter den Forschungseinrichtungen gibt es mehr als ein Viertel (26\%), die ihre Hauptaufgabe im Projekt in der Entwicklung eigener Produkte, Verfahren oder Dienstleistungen sieht. Bei der Hälfte der Forschungseinrichtungen werden die FuE-Aufgaben jedoch mit Blick auf die Unterstützung der Entwicklung von Produkten, Verfahren oder Dienstleistungen bei Partnern durchgeführt. Auch einzelne (Pilot-)Anwender finden sich unter den Forschungsreinrichtungen. Etwa $10 \%$ der Antwortenden aus Forschungseinrichtungen gaben an, mit der FuE im Projekt werde keine unmittelbare wirtschaftliche Verwertung angestrebt.

Unternehmen in Projekten der Gemeinsamen Technologieinitiativen waren zu einem besonders hohen Anteil mit FuE zur Entwicklung ihrer eigenen Produkte, Verfahren oder Dienstleistungen beschäftigt (20 von 23 Unternehmen). In den nationalen IKT der Zukunft-Projekten und in Projekten zum Demographischen Wandel liegt der Anteil der Unternehmen, der in den 
Projekten überwiegend FuE zur Entwicklung ihrer eigenen Produkte, Verfahren oder Dienstleistungen betreibt, bei etwa der Hälfte der Geförderten.

Die meisten Partnerschaften können bereits auf eine frühere FuE-Zusammenarbeit zurückblicken. Unter den Unternehmen finden sich $78 \%$, die bereits vor dem Projekt mit einer oder mehreren beteiligten Forschungsreinrichtungen in anderen FuE-Projekten zusammengearbeitet haben und $60 \%$, die dies bereits mit anderen, an den Projekten beteiligten entwickelnden Unternehmen taten. Die Anteile unter den Forschungseinrichtungen liegen auf ähnlichem Niveau. Sieben von zehn Forschungsreinrichtungen (70 \%) hatten schon mit anderen Forschungsreinrichtungen im Projekt zuvor zusammengearbeitet und $63 \%$ mit am Projekt beteiligten Unternehmen.

Abbildung 16 Vorerfahrungen mit Partnern: Anteil der Antwortenden, die angaben, ihre Einrichtung habe bereits vor dem Projekt mit einem oder mehreren Partnern zu anderen FuE-Themen zusammengearbeitet, nach Teilnehmergruppen

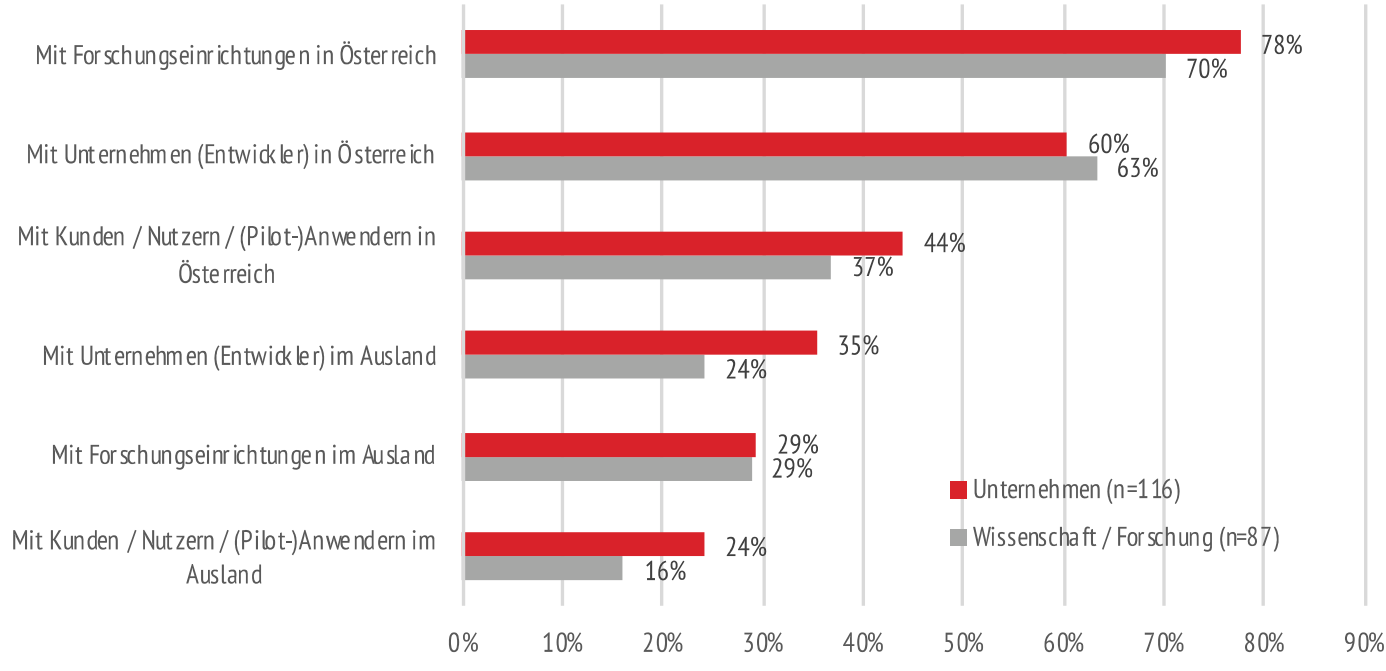

Quelle: Befragung Evaluierung BMVIT-IKT-Programme 2017; inspire research

Bezüglich der Vorerfahrungen in den Projekten zeigen sich Unterschiede zwischen den Förderthemen: In Projekten der Gemeinsamen Technologieinitiativen hatten die Partner besonders häufig bereits zuvor mit anderen Entwicklungspartnern und Forschungseinrichtungen im In- und Ausland zusammengearbeitet. Ein deutlich höherer Anteil an neuen FuE-Kooperationen beobachten wir hingegen bei den Projekten zum Demographischen Wandel.

Tabelle 19 Vorerfahrungen mit Partnern: Anteil der Antwortenden, die angaben, ihre Einrichtung habe bereits vor dem Projekt mit einem oder mehreren Partnern zu anderen FuE-Themen zusammengearbeitet, nach Förderthemen und Teilnehmergruppen

\begin{tabular}{|c|c|c|c|c|c|c|}
\hline & \multicolumn{2}{|c|}{ IKT der Zukunft } & \multicolumn{2}{|c|}{ GTI } & \multicolumn{2}{|c|}{ Dem. Wandel } \\
\hline & $\begin{array}{l}\text { Wissen- } \\
\text { schaft } \\
(\mathrm{N}=42)\end{array}$ & $\begin{array}{c}\text { Unter- } \\
\text { nehmen } \\
(\mathrm{N}=64)\end{array}$ & $\begin{array}{l}\text { Wissen- } \\
\text { schaft } \\
(\mathrm{N}=23)\end{array}$ & $\begin{array}{c}\text { Unter- } \\
\text { nehmen } \\
(\mathrm{N}=22)\end{array}$ & $\begin{array}{l}\text { Wissen- } \\
\text { schaft } \\
(\mathrm{N}=23)\end{array}$ & $\begin{array}{c}\text { Unter- } \\
\text { nehmen } \\
(\mathrm{N}=30)\end{array}$ \\
\hline Mit Forschungseinrichtungen in Österreich & $69 \%$ & $81 \%$ & $91 \%$ & $82 \%$ & $48 \%$ & $67 \%$ \\
\hline Mit Forschungseinrichtungen im Ausland & $14 \%$ & $23 \%$ & $65 \%$ & $59 \%$ & $17 \%$ & $20 \%$ \\
\hline $\begin{array}{l}\text { Mit Kunden / Nutzern / (Pilot-)Anwendern } \\
\text { in Österreich }\end{array}$ & $31 \%$ & $52 \%$ & $43 \%$ & $23 \%$ & $39 \%$ & $43 \%$ \\
\hline $\begin{array}{l}\text { Mit Kunden / Nutzern / (Pilot-)Anwendern } \\
\text { im Ausland }\end{array}$ & $14 \%$ & $25 \%$ & $22 \%$ & $36 \%$ & $13 \%$ & $13 \%$ \\
\hline $\begin{array}{l}\text { Mit Unternehmen (Entwickler) in Öster- } \\
\text { reich }\end{array}$ & $60 \%$ & $63 \%$ & $87 \%$ & $68 \%$ & $43 \%$ & $50 \%$ \\
\hline Mit Unternehmen (Entwickler) im Ausland & $14 \%$ & $31 \%$ & $48 \%$ & $59 \%$ & $17 \%$ & $27 \%$ \\
\hline
\end{tabular}


Quelle: Befragung Evaluierung der BMVIT IKT-Programme 2017; Auswertung inspire research

In den meisten Fällen wurden die gemeinsamen Vorprojekte ebenfalls bereits aus nationalen FuE-Förderungen (teil-)finanziert. Insgesamt $80 \%$ der Unternehmen und $90 \%$ der wissenschaftlichen Partner in den Projekten gaben nationale FuE-Förderungen als Finanzierungsquellen bei früheren FuE-Projekten mit den Partnern an. Zwei Drittel der Unternehmen (67\%) und $57 \%$ der Forschungseinrichtungen führten mit einzelnen Partnern zuvor bereits aus europäischen FuE-Mitteln finanzierte Projekte durch. Fast die Hälfte der Unternehmen (46\%) war zuvor an gemeinsamen Projekten beteiligt, die ganz aus Eigenmitteln finanziert wurden. Außerdem gaben $39 \%$ der Unternehmen an, dass sie für die frühere FuE-Zusammenarbeit mit Partnern keine gesonderte Finanzierung benötigt hätten, da die FuE-Zusammenarbeit Teil des Tagesgeschäfts gewesen sei.

Abbildung 17 Finanzierung früherer FuE-Zusammenarbeit: Anteil der Antwortenden, die angaben, ihre Einrichtung habe die frühere FuE-Zusammenarbeit mit Partnern aus den folgenden Quellen finanziert, nach Teilnehmergruppen

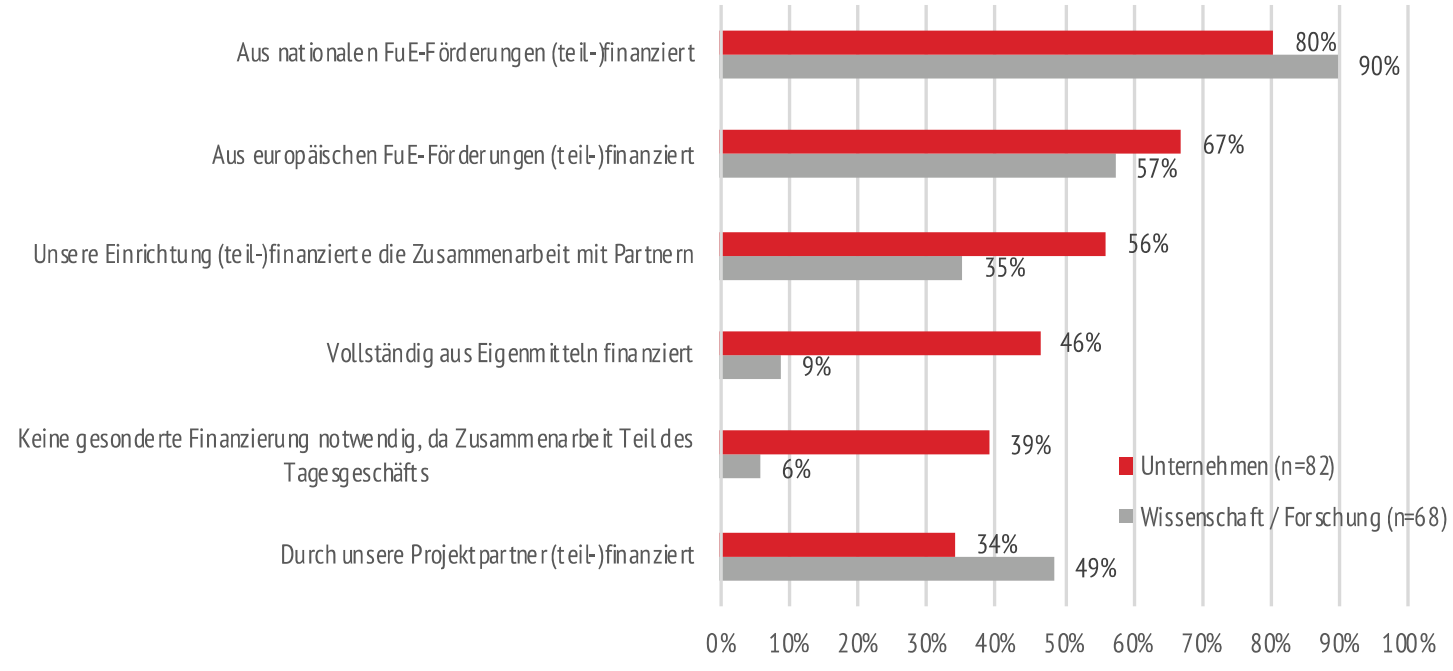

Quelle: Befragung Evaluierung BMVIT-IKT-Programme 2017; inspire research

Die beteiligen Forschungseinrichtungen finanzierten hingegen frühere Kooperationen mit den Partnern nur zu einem geringen Anteil ganz aus Eigenmitteln ( 9 \%) oder ohne gesonderte Finanzierung als Teil des Tagesgeschäfts (6\%). Über eine (Teil-)Finanzierung von früheren FuE-Projekten durch die Partner berichteten fast die Hälfte (49\%) der Forschungseinrichtungen. Interessanterweise brachten die Forschungseinrichtungen trotzdem in signifikantem Umfang Eigenleistungen in frühere FuE-Kooperationsprojekte mit den Partnern ein. Immerhin mehr als ein Drittel (35\%) der wissenschaftlichen Partner gab an, die frühere Zusammenarbeit mit den Partnern selbst teilfinanziert zu haben, also auch Leistungen erbracht zu haben, die nicht durch die Finanzierungsbeiträge der Projektpartner oder durch öffentliche Förderungen gedeckt waren.

Die Partner in Projekten der Gemeinsamen Technologieinitiativen gaben am häufigsten an, frühere FuE-Zusammenarbeit mit den Partnern aus europäischen FuE-Förderungen finanziert zu haben. Bei Projekten zum Demographischen Wandel ist der Anteil der wissenschaftlichen Partner, die in früheren Projekten eine (Teil-)Finanzierung durch ihre Projektpartner erhalten hatten im Vergleich zu Projekten in den zwei anderen Förderthemen besonders gering. Dies interpretieren wir als Hinweis, dass diese Projekte tatsächlich häufig auf eine öffentliche Finanzierung angewiesen sind, um durchgeführt zu werden. 
Tabelle 20 Finanzierung früherer FuE-Zusammenarbeit: Anteil der Antwortenden, die angaben, ihre Einrichtung habe die frühere FuE-Zusammenarbeit mit Partnern aus den folgenden Quellen finanziert, nach Förderthemen und Teilnehmergruppen

\begin{tabular}{|c|c|c|c|c|c|c|}
\hline & \multicolumn{2}{|c|}{ IKT der Zukunft } & \multicolumn{2}{|c|}{ GTI } & \multicolumn{2}{|c|}{ Dem. Wandel } \\
\hline & $\begin{array}{l}\text { Wissen- } \\
\text { schaft } \\
(\mathrm{N}=32)\end{array}$ & $\begin{array}{l}\text { Unter- } \\
\text { nehmen } \\
(\mathrm{N}=48)\end{array}$ & $\begin{array}{l}\text { Wissen- } \\
\text { schaft } \\
(\mathrm{N}=23)\end{array}$ & $\begin{array}{l}\text { Unter- } \\
\text { nehmen } \\
(\mathrm{N}=21)\end{array}$ & $\begin{array}{l}\text { Wissen- } \\
\text { schaft } \\
(\mathrm{N}=13)\end{array}$ & $\begin{array}{l}\text { Unter- } \\
\text { nehmen } \\
(\mathrm{N}=16)\end{array}$ \\
\hline $\begin{array}{l}\text { Keine gesonderte Finanzierung notwendig, } \\
\text { da Zusammenarbeit Teil des Tagesge- } \\
\text { schäfts }\end{array}$ & $6 \%$ & $40 \%$ & $4 \%$ & $38 \%$ & $8 \%$ & $31 \%$ \\
\hline Vollständig aus Eigenmitteln finanziert & $16 \%$ & $44 \%$ & $0 \%$ & $52 \%$ & $8 \%$ & $38 \%$ \\
\hline $\begin{array}{l}\text { Aus nationalen FuE-Förderungen (teil-) fi- } \\
\text { nanziert }\end{array}$ & $84 \%$ & $88 \%$ & $91 \%$ & $67 \%$ & $100 \%$ & $63 \%$ \\
\hline $\begin{array}{l}\text { Aus europäischen FuE-Förderungen (teil-) } \\
\text { finanziert }\end{array}$ & $41 \%$ & $56 \%$ & $78 \%$ & $81 \%$ & $62 \%$ & $69 \%$ \\
\hline $\begin{array}{l}\text { Durch unsere Projektpartner (teil-) finan- } \\
\text { ziert }\end{array}$ & $56 \%$ & $42 \%$ & $52 \%$ & $19 \%$ & $23 \%$ & $25 \%$ \\
\hline $\begin{array}{l}\text { Unsere Einrichtung (teil-)finanzierte die } \\
\text { Zusammenarbeit mit Partnern }\end{array}$ & $38 \%$ & $52 \%$ & $30 \%$ & $62 \%$ & $38 \%$ & $50 \%$ \\
\hline
\end{tabular}

Quelle: Befragung Evaluierung der BMVIT IKT-Programme 2017; Auswertung inspire research

In welchen Phasen der geförderten Projekte engagieren sich die beteiligten Unternehmen und Forschungseinrichtungen am stärksten? Bei den Forschungseinrichtungen steht eindeutig die Durchführung der Forschungsarbeiten im Vordergrund. Insgesamt 93\% der Forschungseinrichtungen waren an der Durchführung der Forschungsarbeiten in großem oder sehr großem Umfang beteiligt. Auch der wissenschaftlichen Ergebnisverwertung widmen sich die Forschungsreinrichtungen sehr häufig in großem oder sehr großem Umfang (87\%). Deutlich häufiger als die Unternehmenspartner kümmern sich die Forschungseinrichtungen um den Ergebnistransfer bzw. die Öffentlichkeitsarbeit im Projekt. Außerdem sind sie in gröBerem Umfang als die Unternehmen mit der Koordinierung der Partner und der Festlegung der Arbeitsprogramme befasst.

Abbildung 18 Beteiligung der Partner im Projekt: Anteil der Antwortenden, die angaben, ihre Einrichtung sei an den folgenden Phasen des Projekts im „großen“ bzw. „sehr großen“ Umfang beteiligt gewesen, nach Teilnehmergruppen

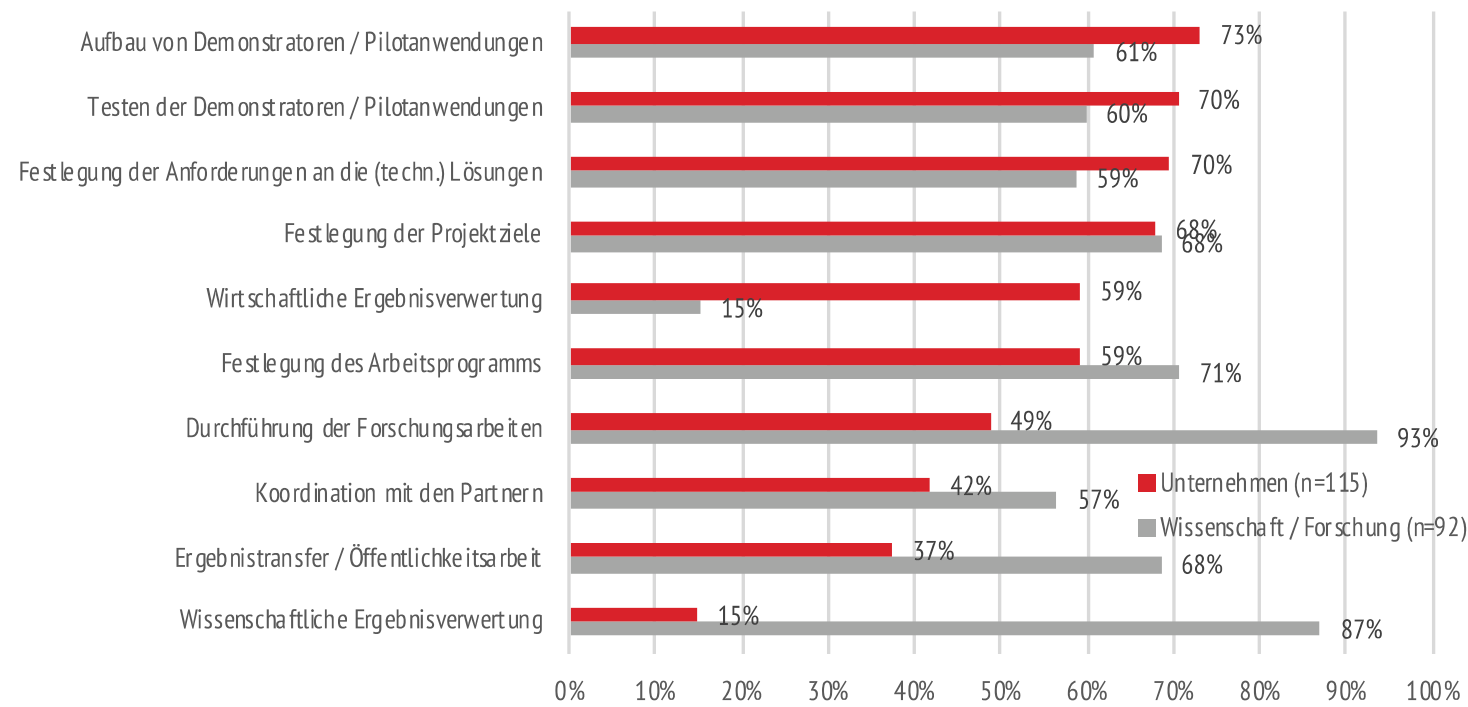

Quelle: Befragung Evaluierung BMVIT-IKT-Programme 2017; inspire research 
Die Unternehmen beteiligen sich hingegen stärker am Aufbau und am Testen von Demonstratoren und Pilotanwendungen, sowie an der Festlegung der Anforderungen an die zu erarbeitenden Lösungen im Projekt. Wenig überraschend dominieren Unternehmen die wirtschaftliche Ergebnisverwertung. Gleichzeitig fällt hierbei jedoch auf, dass immerhin $41 \%$ der geförderten Unternehmen nicht, im geringen oder nur in mäßigem Umfang an der wirtschaftlichen Verwertung der Ergebnisse beteiligt waren bzw. sind. An der Festlegung der Projektziele waren Unternehmen und Forschungseinrichtungen ungefähr zu gleichen Anteilen in "großem" oder "sehr großem" Umfang beteiligt.

Zwischen Projekten in verschiedenen Förderthemen zeigen sich wieder deutliche Unterschiede hinsichtlich des Umfangs der Beteiligung der Partner. Die Projekte in den nationalen IKT der Zukunft-Ausschreibungen werden am deutlichsten von den wissenschaftlichen Partnern dominiert. Nur bezüglich der wirtschaftlichen Ergebnisverwertung und des Testens von Demonstratoren und Pilotanwendungen liegt der Anteil der Unternehmen, die in diesen Projektphasen in großem oder sehr großem Umfang an den Arbeiten beteiligt sind, über dem entsprechenden Anteil unter den Forschungseinrichtungen. Hingegen sind die Projekte der Gemeinsamen Technologieinitiativen sehr deutlich unternehmensgetrieben. Bis auf die Durchführung der Forschungsarbeiten, den Ergebnistransfer / Öffentlichkeitsarbeit und die wissenschaftliche Ergebnisverwertung dominieren die Unternehmen alle Projektphasen. Deutlich ausgeglichener ist die Rollenverteilung in Projekten zum Demographischen Wandel. Unternehmen und Forschungsreinrichtungen engagieren sich in diesen Projekten in hohem Umfang in fast allen Projektphasen gemeinsam. Auffällig sind hier insbesondere die hohen Anteile an Unternehmen, die Koordinierungsaufgaben im Projekt in großem oder sehr groBem Umfang wahrnehmen (60\%) und die sich auch um den Ergebnistransfer / Öffentlichkeitsarbeit (57\%) und die wissenschaftliche Ergebnisverwertung (27\%) kümmern.

Tabelle 21 Beteiligung der Partner im Projekt: Anteil der Antwortenden, die angaben, ihre Einrichtung sei an den folgenden Phasen des Projekts im "großen" bzw. „sehr großen“ Umfang beteiligt gewesen, nach Förderthemen und Teilnehmergruppen

\begin{tabular}{|c|c|c|c|c|c|c|}
\hline & \multicolumn{2}{|c|}{ IKT der Zukunft } & \multicolumn{2}{|c|}{ GTI } & \multicolumn{2}{|c|}{ Dem. Wandel } \\
\hline & $\begin{array}{l}\text { Wissen- } \\
\text { schaft } \\
(\mathrm{N}=45)\end{array}$ & $\begin{array}{l}\text { Unter- } \\
\text { nehmen } \\
(\mathrm{N}=63)\end{array}$ & $\begin{array}{l}\text { Wissen- } \\
\text { schaft } \\
(\mathrm{N}=24)\end{array}$ & $\begin{array}{l}\text { Unter- } \\
\text { nehmen } \\
(\mathrm{N}=23)\end{array}$ & $\begin{array}{l}\text { Wissen- } \\
\text { schaft } \\
(\mathrm{N}=23)\end{array}$ & $\begin{array}{l}\text { Unter- } \\
\text { nehmen } \\
(\mathrm{N}=30)\end{array}$ \\
\hline Festlegung der Projektziele & $89 \%$ & $78 \%$ & $25 \%$ & $52 \%$ & $74 \%$ & $57 \%$ \\
\hline Festlegung des Arbeitsprogramms & $96 \%$ & $62 \%$ & $21 \%$ & $48 \%$ & $74 \%$ & $60 \%$ \\
\hline Koordination mit den Partnern & $71 \%$ & $35 \%$ & $25 \%$ & $35 \%$ & $61 \%$ & $60 \%$ \\
\hline $\begin{array}{l}\text { Festlegung der Anforderungen an die } \\
\text { (technischen) Lösungen }\end{array}$ & $78 \%$ & $75 \%$ & $29 \%$ & $57 \%$ & $52 \%$ & $67 \%$ \\
\hline Durchführung der Forschungsarbeiten & $98 \%$ & $38 \%$ & $88 \%$ & $74 \%$ & $91 \%$ & $50 \%$ \\
\hline $\begin{array}{l}\text { Aufbau von Demonstratoren / Pilotanwen- } \\
\text { dungen }\end{array}$ & $67 \%$ & $65 \%$ & $54 \%$ & $78 \%$ & $57 \%$ & $50 \%$ \\
\hline $\begin{array}{l}\text { Testen der Demonstratoren / Pilotanwen- } \\
\text { dungen }\end{array}$ & $69 \%$ & $73 \%$ & $38 \%$ & $61 \%$ & $65 \%$ & $70 \%$ \\
\hline Wirtschaftliche Ergebnisverwertung & $16 \%$ & $63 \%$ & $13 \%$ & $57 \%$ & $17 \%$ & $50 \%$ \\
\hline Wissenschaftliche Ergebnisverwertung & $93 \%$ & $10 \%$ & $83 \%$ & $13 \%$ & $78 \%$ & $27 \%$ \\
\hline Ergebnistransfer / Öffentlichkeitsarbeit & $84 \%$ & $35 \%$ & $42 \%$ & $17 \%$ & $65 \%$ & $57 \%$ \\
\hline
\end{tabular}

Quelle: Befragung Evaluierung der BMVIT IKT-Programme 2017; Auswertung inspire research

Unternehmen arbeiten in den Projekten am intensivsten mit Forschungseinrichtungen zusammen, Forschungseinrichtungen am intensivsten mit den Unternehmen. Etwas weniger häufig kooperieren Unternehmen mit anderen entwickelnden Unternehmen intensiv oder sehr intensiv in den Projekten. 
Abbildung 19 Intensität der Zusammenarbeit: Anteil der Antwortenden, die angaben, ihre Einrichtung habe mit anderen Partnern im Projekt „intensiv“ bzw. „sehr intensiv“ zusammengearbeitet, nach Teilnehmergruppen

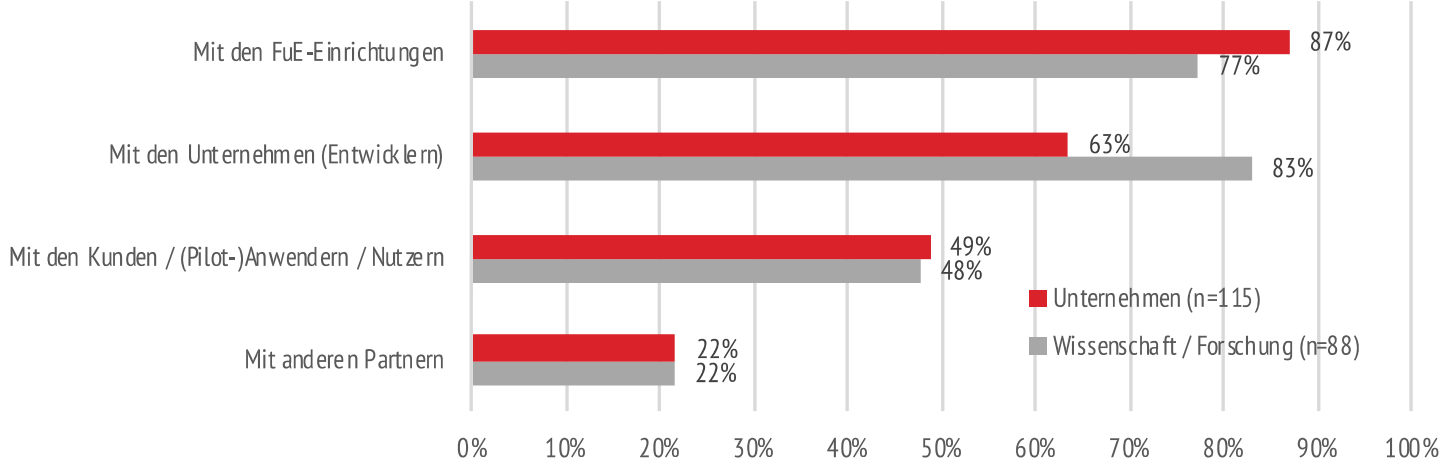

Quelle: Befragung Evaluierung BMVIT-IKT-Programme 2017; inspire research

Bezüglich der Intensität der Zusammenarbeit zeigen sich wieder deutliche Unterschiede zwischen Projekten unterschiedlicher Förderthemen. In Projekten der Gemeinsamen Technologieinitiativen ist die Intensität der Zusammenarbeit von Unternehmen mit Forschungseinrichtungen vergleichsweise gering. Gleichzeitig spielen für die Unternehmen andere (internationale) Partner hier eine deutlich größere Rolle bei der Zusammenarbeit im Projekt.

Tabelle 22 Intensität der Zusammenarbeit: Anteil der Antwortenden, die angaben, ihre Einrichtung hätte mit anderen Partnern im Projekt „intensiv“ bzw. „sehr intensiv“ zusammengearbeitet, nach Förderthemen und Teilnehmergruppen

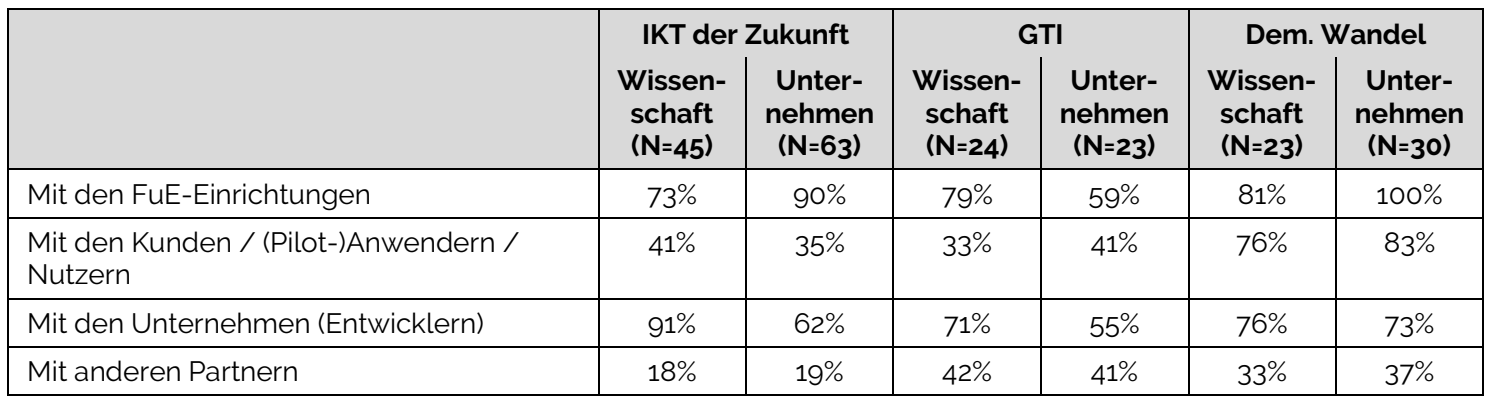

Quelle: Befragung Evaluierung der BMVIT IKT-Programme 2017; Auswertung inspire research

Demgegenüber zeichnen sich Projekte zum Demographischen Wandel in sehr hohem Ausmaß durch eine intensive oder sehr intensive Zusammenarbeit zwischen Unternehmen und Forschungseinrichtungen aus. Insbesondere die Intensität der Zusammenarbeit mit Kunden, (Pilot)Anwendern und Nutzern liegt deutlich über dem Niveau von Projekten in den beiden anderen Förderthemen. Den benefit- und AAL-Projekten gelingt es offenbar, ihrem Anspruch nach aktiver Einbeziehung von Nutzern und (Pilot-)Anwendern auch gerecht zu werden.

\subsection{Probleme bei der Projektdurchführung}

Mit welchen Problemen und Herausforderungen sind die Projektpartner bei der Durchführung ihrer Vorhaben konfrontiert? Die Angaben der Ansprechpartner/innen in der Befragung zeigen, dass es vor allem technische Probleme und organisatorische Probleme (wie der Ausfall von Partnern) sind, die den Projektfortschritt bzw. die Erreichung der Projektziele behindern. Andere Probleme werden von den Projektpartnern deutlich weniger häufig wahrgenommen. 
Abbildung 20 Probleme, die den Projektfortschritt oder die Erreichung der Projektziele behindern: Anteil der Antwortenden, die das Auftreten der folgenden Probleme angaben, nach Teilnehmergruppen

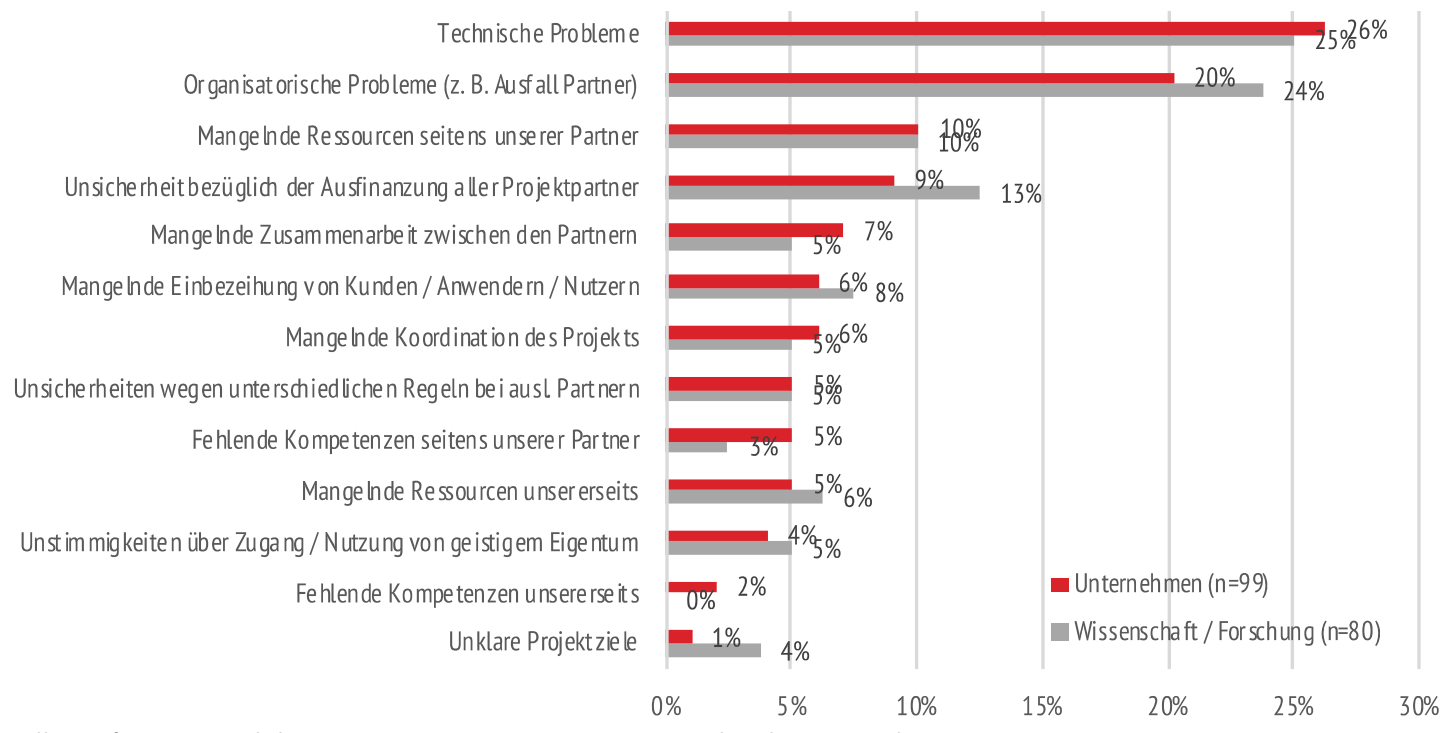

Quelle: Befragung Evaluierung BMVIT-IKT-Programme 2017; inspire research

Nur bei Projekten zum Demographischen Wandel gibt es vor allem aus Sicht der beteiligten Unternehmen ein deutlich breiteres Set von Faktoren, das bei der Durchführung der Vorhaben Probleme bereitet.

Tabelle 23 Probleme, die den Projektfortschritt oder die Erreichung der Projektziele behindern: Anteil der Antwortenden, die das Auftreten der folgenden Probleme angaben, nach Förderthemen und Teilnehmergruppen

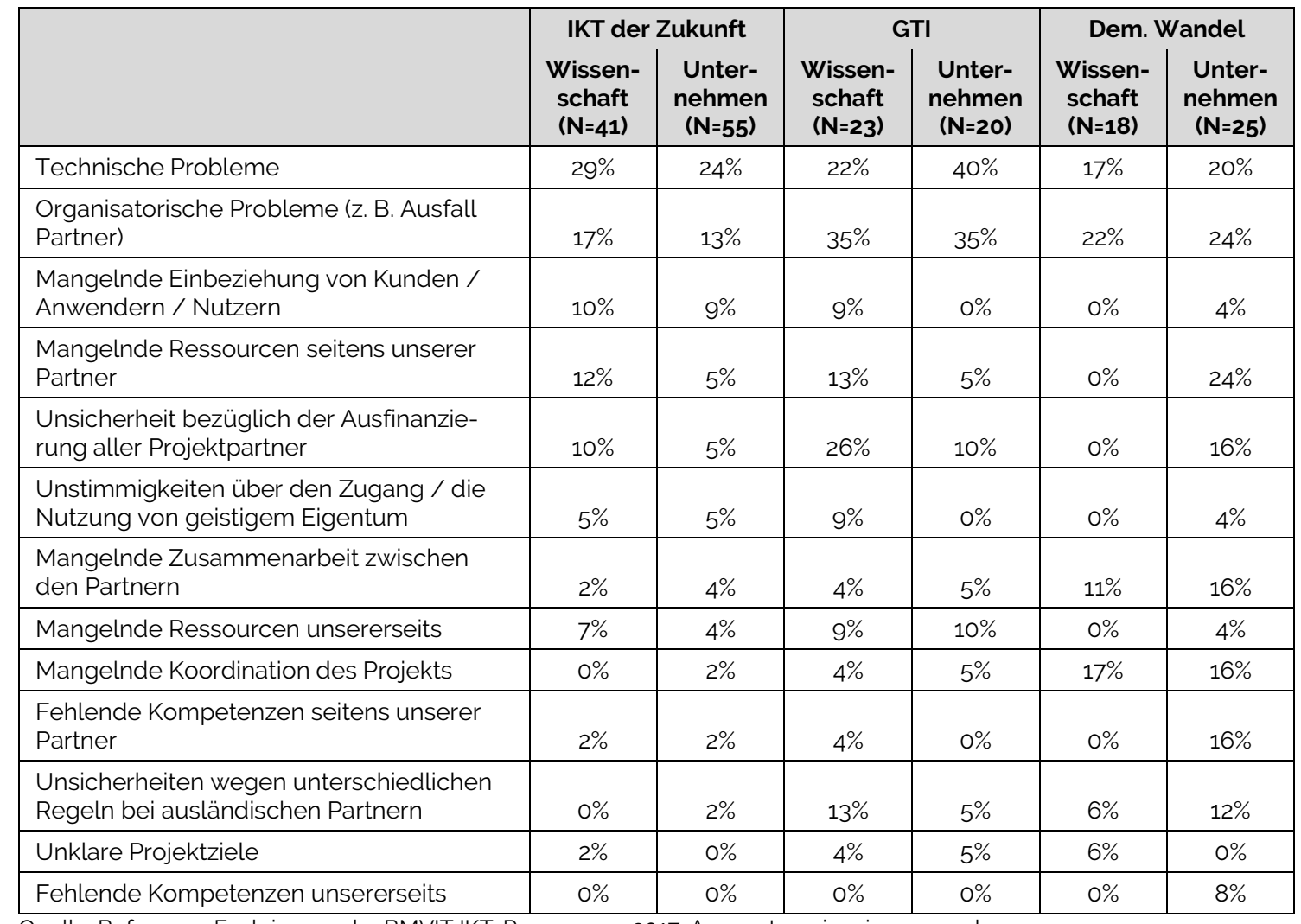

Quelle: Befragung Evaluierung der BMVIT IKT-Programme 2017; Auswertung inspire research 
Häufiger angegeben als in Projekten zu anderen Förderthemen werden von den Unternehmen beispielsweise die Faktoren "Mangelnde Ressourcen seitens unserer Partner", "Mangelnde Zusammenarbeit zwischen den Partnern" und "Mangelnde Koordination des Projekts". Interessant ist in diesem Zusammenhang, dass es bei Projekten zum Demographischen Wandel vor allem die Unternehmen sind, die Probleme hinsichtlich mangelnder Ressourcen und Kompetenzen ihrer Partner wahrnehmen. Die beteiligten Unternehmen dürften also mit inhaltlichen Erwartungen in die Projekte gehen, die von ihren Partnern häufig nicht erfüllt werden können. Die wissenschaftlichen Einrichtungen in Projekten zum Demographischen Wandel sehen hingegen in erster Linie organisatorische und Koordinationsherausforderungen, weniger jedoch Kompetenz- oder Ressourcenprobleme.

\subsection{Zufriedenheit der Förderempfänger mit der Programmdurchführung}

Sowohl unsere Gespräche mit Programmteilnehmer/inne/n als auch die Antworten der Ansprechpersonen in der Befragung zeigen ein insgesamt sehr hohes Ausmaß an Zufriedenheit mit dem Programm und seiner Durchführung und Abwicklung. Die Unternehmen sind insbesondere mit den Anforderungen an die Zusammensetzung der Projektkonsortien und mit den thematischen Schwerpunkten der Ausschreibungen zufrieden oder sehr zufrieden. Ein hohes Ausmaß an Zufriedenheit gibt es unter den Unternehmen auch hinsichtlich des Zahlungsplans der Förderungen und der Anforderungen an das Berichtswesen bei der FFG. Unzufrieden sind die Unternehmen vor allem bezüglich der Dauer der administrativen Prozesse und des Umfangs bzw. der Komplexität des Antragsverfahrens.

Abbildung 21 Zufriedenheit mit den administrativen Verfahren und Abläufen im Projekt: Anteil der Antwortenden, die mit folgenden Aspekten „sehr zufrieden“ bzw. „zufrieden“ waren, nach Teilnehmergruppen

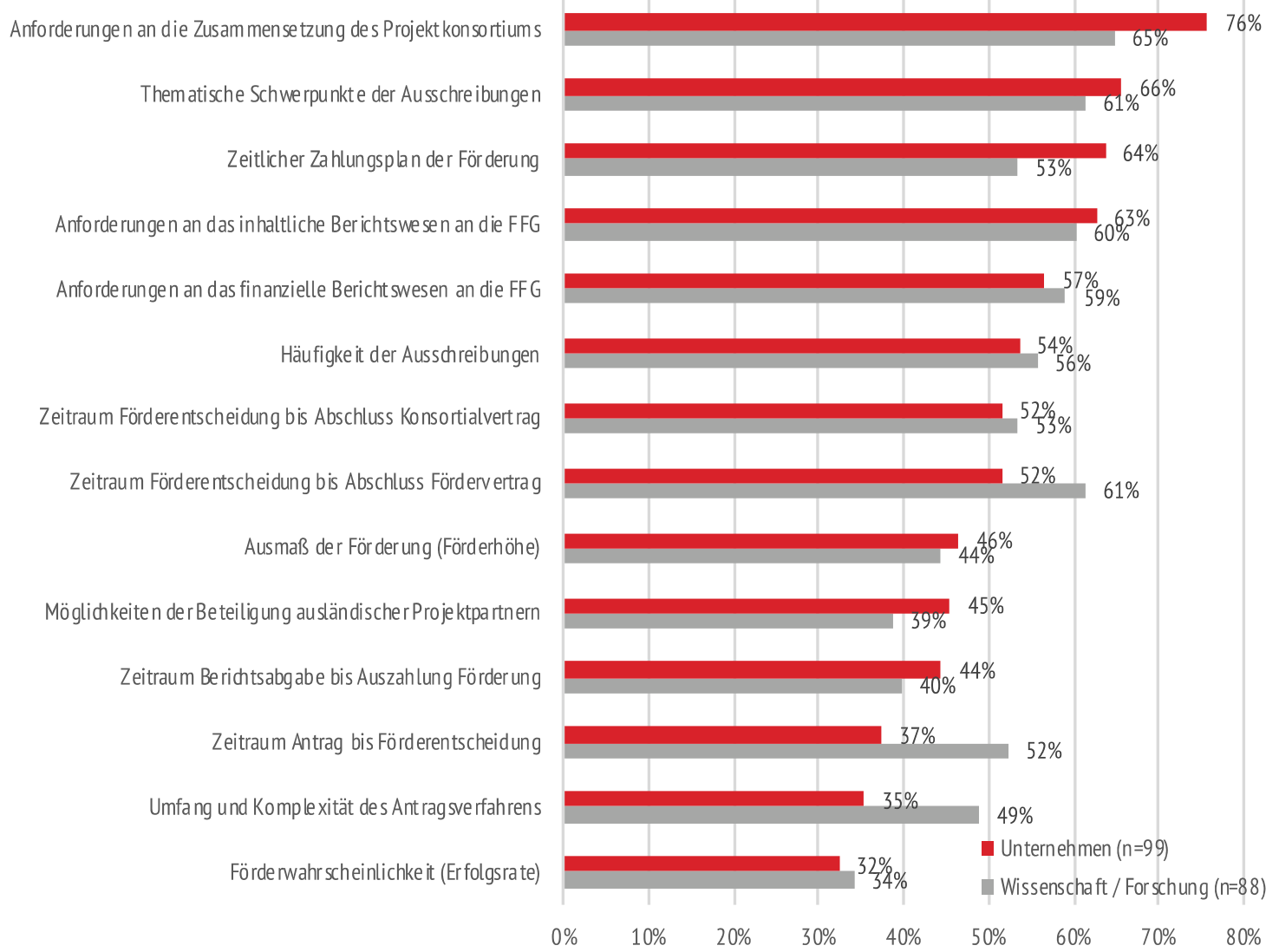

Quelle: Befragung Evaluierung der BMVIT IKT-Programme 2017; Auswertung inspire research 
Die beteiligten Forschungsreinrichtungen zeigen insgesamt ein mehr ausgeglichenes Aus$\mathrm{maß}$ an Zufriedenheit mit den administrativen Verfahren und Abläufen als die Unternehmen. Deutlich positiver als die Unternehmen schätzen die wissenschaftlichen Einrichtungen die Dauer der Verfahren sowie Umfang und Komplexität der Anträge ein.

Die Unternehmen sind vor allem in Projekten der Gemeinsamen Technologieinitiativen mit den thematischen Schwerpunkten der Ausschreibungen sowie mit den Anforderungen an das Berichtswesen besonders zufrieden. Die größte Herausforderung in diesen Projekten sehen die Unternehmen im Abschluss des Konsortialvertrags nach bereits vorliegender Förderentscheidung. Auch mit dem Zeitraum bis zur Auszahlung der Förderungen sind viele Partner in Projekten der Gemeinsamen Technologieinitiativen eher unzufrieden. Auffällig ist schließlich, dass die Forschungseinrichtungen und Unternehmen in Projekten der Gemeinsamen Technologieinitiativen mit der Förderhöhe besonders unzufrieden sind. Dies, obwohl es gerade diese Projekte sind, die im Vergleich zu Projekten zu anderen Förderthemen den Partnern absolut die höchsten Förderbeträge zur Verfügung stellen.

Tabelle 24 Zufriedenheit mit den administrativen Verfahren und Abläufen im Projekt: Anteil der Antwortenden, die mit folgenden Aspekten „sehr zufrieden“ bzw. „zufrieden“ waren, nach Förderthemen und Teilnehmergruppen

\begin{tabular}{|c|c|c|c|c|c|c|}
\hline & \multicolumn{2}{|c|}{ IKT der Zukunft } & \multicolumn{2}{|c|}{ GTI } & \multicolumn{2}{|c|}{ Dem. Wandel } \\
\hline & $\begin{array}{c}\text { Wissen- } \\
\text { schaft } \\
(\mathrm{N}=46)\end{array}$ & $\begin{array}{c}\text { Unter- } \\
\text { nehmen } \\
(N=56)\end{array}$ & $\begin{array}{c}\text { Wissen- } \\
\text { schaft } \\
(\mathrm{N}=23)\end{array}$ & $\begin{array}{c}\text { Unter- } \\
\text { nehmen } \\
(\mathrm{N}=\mathbf{2 0})\end{array}$ & $\begin{array}{c}\text { Wissen- } \\
\text { schaft } \\
(\mathrm{N}=20)\end{array}$ & $\begin{array}{c}\text { Unter- } \\
\text { nehmen } \\
(\mathrm{N}=\mathbf{2 4})\end{array}$ \\
\hline Häufigkeit der Ausschreibungen & $54 \%$ & $54 \%$ & $43 \%$ & $65 \%$ & $70 \%$ & $42 \%$ \\
\hline $\begin{array}{l}\text { Thematische Schwerpunkte der Aus- } \\
\text { schreibungen }\end{array}$ & $54 \%$ & $61 \%$ & $61 \%$ & $85 \%$ & $75 \%$ & $58 \%$ \\
\hline $\begin{array}{l}\text { Möglichkeiten der Beteiligung ausländi- } \\
\text { scher Projektpartnern }\end{array}$ & $17 \%$ & $29 \%$ & $91 \%$ & $90 \%$ & $25 \%$ & $46 \%$ \\
\hline $\begin{array}{l}\text { Anforderungen an die Zusammensetzung } \\
\text { Projektkonsortium }\end{array}$ & $67 \%$ & $79 \%$ & $61 \%$ & $70 \%$ & $60 \%$ & $71 \%$ \\
\hline $\begin{array}{l}\text { Umfang und Komplexität des Antragsver- } \\
\text { fahrens }\end{array}$ & $50 \%$ & $34 \%$ & $30 \%$ & $25 \%$ & $65 \%$ & $46 \%$ \\
\hline Zeitraum Antrag bis Förderentscheidung & $52 \%$ & $43 \%$ & $43 \%$ & $20 \%$ & $60 \%$ & $38 \%$ \\
\hline Förderwahrscheinlichkeit (Erfolgsrate) & $28 \%$ & $30 \%$ & $48 \%$ & $25 \%$ & $30 \%$ & $42 \%$ \\
\hline Ausmaß der Förderung (Förderhöhe) & $52 \%$ & $59 \%$ & $13 \%$ & $15 \%$ & $60 \%$ & $42 \%$ \\
\hline $\begin{array}{l}\text { Zeitraum Förderentscheidung bis Ab- } \\
\text { schluss Fördervertrag }\end{array}$ & $83 \%$ & $57 \%$ & $26 \%$ & $40 \%$ & $60 \%$ & $46 \%$ \\
\hline $\begin{array}{l}\text { Zeitraum Förderentscheidung bis Ab- } \\
\text { schluss Konsortialvertrag }\end{array}$ & $70 \%$ & $55 \%$ & $13 \%$ & $25 \%$ & $60 \%$ & $63 \%$ \\
\hline $\begin{array}{l}\text { Anforderungen an das inhaltliche Berichts- } \\
\text { wesen an die FFG }\end{array}$ & $67 \%$ & $64 \%$ & $52 \%$ & $80 \%$ & $50 \%$ & $46 \%$ \\
\hline $\begin{array}{l}\text { Anforderungen an das inhaltliche Berichts- } \\
\text { wesen an andere Stellen }\end{array}$ & $11 \%$ & $23 \%$ & $57 \%$ & $65 \%$ & $15 \%$ & $29 \%$ \\
\hline $\begin{array}{l}\text { Anforderungen an das finanzielle Berichts- } \\
\text { wesen FFG }\end{array}$ & $61 \%$ & $57 \%$ & $48 \%$ & $65 \%$ & $65 \%$ & $46 \%$ \\
\hline $\begin{array}{l}\text { Anforderungen an das finanzielle Berichts- } \\
\text { wesen andere Stellen }\end{array}$ & $13 \%$ & $23 \%$ & $39 \%$ & $55 \%$ & $10 \%$ & $21 \%$ \\
\hline Zeitlicher Zahlungsplan der Förderung & $59 \%$ & $73 \%$ & $30 \%$ & $50 \%$ & $65 \%$ & $50 \%$ \\
\hline $\begin{array}{l}\text { Zeitraum Berichtsabgabe bis Auszahlung } \\
\text { der Förderung }\end{array}$ & $41 \%$ & $52 \%$ & $26 \%$ & $30 \%$ & $50 \%$ & $38 \%$ \\
\hline
\end{tabular}

Quelle: Befragung Evaluierung der BMVIT IKT-Programme 2017; Auswertung inspire research

Bei den zum Teil deutlich kleineren Projekten in den IKT der Zukunft-Ausschreibungen und zum Demographischen Wandel sind die beteiligten Einrichtungen hingegen mit den finanzi- 
ellen Aspekten der Programmdurchführung deutlich zufriedener. Lediglich die Förderwahrscheinlichkeit wird von den wissenschaftlichen Einrichtungen in Projekten der IKT der Zukunft-Ausschreibungen und zum Demographischen Wandel kritischer gesehen als in Projekten der Gemeinsamen Technologieinitiativen.

Auch mit der Beratung und Unterstützung durch die FFG sind die Programmteilnehmer insgesamt in hohem Ausmaß zufrieden bzw. sehr zufrieden. Am positivsten bewerten die geförderten Unternehmen die Schnelligkeit der Fragebeantwortungen und der Rückmeldungen der FFG sowie die fachliche Kompetenz der Ansprechpartner/innen der FFG. Bezüglich der Zufriedenheit mit der Kompetenz der FFG-Mitarbeiter/innen gibt es bei den beteiligten Unternehmen nur geringe Unterschiede zwischen den Förderthemen. Unter den Forschungseinrichtungen sind vor allem jene in den Projekten zum Demographischen Wandel in sehr hohem Ausmaß mit der Kompetenz der FFG-Mitarbeiter/innen "zufrieden“ oder "sehr zufrieden" (94\%).

Die Forschungseinrichtungen sind außerdem in den Förderthemen IKT der Zukunft und Demographischer Wandel mit den allgemeinen Informationen über das Programm in hohem Ausmaß zufrieden. Wissenschaftliche Partner in Projekten der Gemeinsamen Technologieinitiative sind hier in deutlich geringerem Ausmaß zufrieden.

Abbildung 22 Zufriedenheit mit der Beratung und Unterstützung durch die FFG im Vorfeld und während der Durchführung des Projekts: Anteil der Antwortenden, die mit den folgenden Aspekten „sehr zufrieden“ bzw. „zufrieden“ waren, nach Teilnehmergruppen

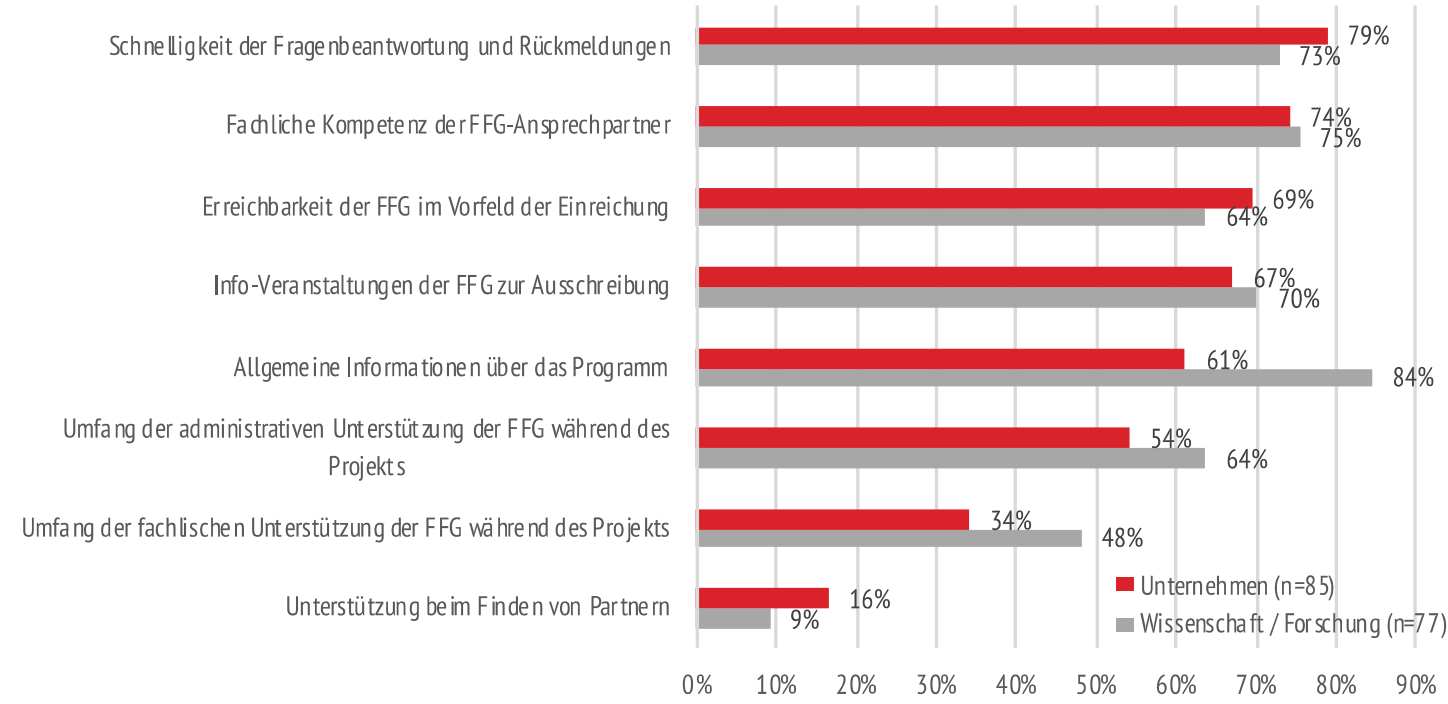

Quelle: Befragung Evaluierung der BMVIT IKT-Programme 2017; Auswertung inspire research

Mehr als zwei Drittel der Programmteilnehmer sind auch mit den angebotenen Informationsveranstaltungen zu den Ausschreibungen „zufrieden“ oder "sehr zufrieden“. Die Informationsveranstaltungen werden insbesondere von den Partnern in den Projekten der nationalen IKT der Zukunft-Ausschreibungen sowie zum Demographischen Wandel sehr geschätzt. Weniger zufrieden sind die Partner in Projekten der Gemeinsamen Technologieinitiativen mit den Informationsveranstaltungen zu den Ausschreibungen.

Das geringste Maß an Zufriedenheit gibt es hinsichtlich des Findens von Partnern für Konsortien. Dieser Aspekt ist jedoch nur für einen Teil der Projekte tatsächlich relevant, etwa bei Vorhaben im AAL-Programm, falls aufgrund von beschränkten Finanzierungsmöglichkeiten Partner aus bestimmten Ländern für die Konsortien gefunden werden müssen. 
Tabelle 25 Zufriedenheit mit der Beratung und Unterstützung durch die FFG im Vorfeld und während der Durchführung des Projekts: Anteil der Antwortenden, die mit den folgenden Aspekten „sehr zufrieden“ bzw. „zufrieden“ waren, nach Förderthemen und Teilnehmergruppen

\begin{tabular}{|l|c|c|c|c|c|c|}
\hline & \multicolumn{2}{|c|}{ IKT der Zukunft } & \multicolumn{2}{|c|}{ GTI } & \multicolumn{2}{c|}{ Dem. Wandel } \\
& $\begin{array}{c}\text { Wissen- } \\
\text { schaft } \\
\mathbf{( N = 4 5 )}\end{array}$ & $\begin{array}{c}\text { Unter- } \\
\text { nehmen } \\
\mathbf{( N = 4 7 )}\end{array}$ & $\begin{array}{c}\text { Wissen- } \\
\text { schaft } \\
\mathbf{( N = 1 7 )}\end{array}$ & $\begin{array}{c}\text { Unter- } \\
\text { nehmen } \\
\mathbf{( N = 2 0 )}\end{array}$ & $\begin{array}{c}\text { Wissen- } \\
\text { schaft } \\
\text { (N=17) }\end{array}$ & $\begin{array}{c}\text { Unter- } \\
\text { nehmen } \\
\text { (N=20) }\end{array}$ \\
\hline $\begin{array}{l}\text { Allgemeine FFG-Informationen über das } \\
\text { Programm }\end{array}$ & $91 \%$ & $87 \%$ & $47 \%$ & $80 \%$ & $94 \%$ & $75 \%$ \\
\hline $\begin{array}{l}\text { Informationsveranstaltungen der FFG zu } \\
\text { den Ausschreibungen }\end{array}$ & $71 \%$ & $72 \%$ & $59 \%$ & $55 \%$ & $82 \%$ & $60 \%$ \\
\hline $\begin{array}{l}\text { FFG-Erreichbarkeit im Vorfeld der Einrei- } \\
\text { chung }\end{array}$ & $69 \%$ & $70 \%$ & $53 \%$ & $60 \%$ & $65 \%$ & $70 \%$ \\
\hline $\begin{array}{l}\text { Schnelligkeit der Fragenbeantwortung } \\
\text { und Rückmeldungen }\end{array}$ & $67 \%$ & $79 \%$ & $71 \%$ & $80 \%$ & $82 \%$ & $70 \%$ \\
\hline $\begin{array}{l}\text { Fachliche Kompetenz der FFG-Ansprech- } \\
\text { partner }\end{array}$ & $64 \%$ & $72 \%$ & $76 \%$ & $70 \%$ & $94 \%$ & $75 \%$ \\
\hline $\begin{array}{l}\text { Umfang der administrativen Unterstützung } \\
\text { durch die FFG während des Projekts }\end{array}$ & $60 \%$ & $70 \%$ & $71 \%$ & $55 \%$ & $71 \%$ & $60 \%$ \\
\hline $\begin{array}{l}\text { Umfang der fachlichen Unterstützung } \\
\text { durch die FFG während des Projekts }\end{array}$ & $40 \%$ & $36 \%$ & $59 \%$ & $25 \%$ & $53 \%$ & $45 \%$ \\
\hline Unterstützung beim Finden von Partnern & $2 \%$ & $11 \%$ & $29 \%$ & $10 \%$ & $6 \%$ & $35 \%$ \\
\hline
\end{tabular}

Quelle: Befragung Evaluierung der BMVIT IKT-Programme 2017; Auswertung inspire research

Auch die Gestaltungs- und Einflussmöglichkeiten auf die Inhalte zukünftiger Ausschreibungen werden von den Förderempfängern insgesamt als gut eingeschätzt. Etwa ein Viertel der Vertreter/innen der Unternehmen und ein Fünftel der technischen Ansprechpartner in Forschungseinrichtungen gab in der Befragung an, dass sie selbst oder ihre Einrichtung an der thematischen Vorbereitung von Ausschreibungen des IKT der Zukunft-Programms beteiligt gewesen seien. Ein weiteres Fünftel der Unternehmen und mehr als ein Drittel der Vertreter/innen der Forschungseinrichtungen waren sich nicht sicher, ob dies auf ihre Einrichtung zutraf (beispielsweise, weil nicht sie selbst, sondern gegebenenfalls Kolleg/inn/en an Vorbereitungsmaßnahmen beteiligt waren).

Abbildung 23 Waren Sie bzw. Ihre Einrichtung an der thematischen Vorbereitung von Ausschreibungen des Programms beteiligt? Anzahl der Antworten nach Teilnehmergruppen

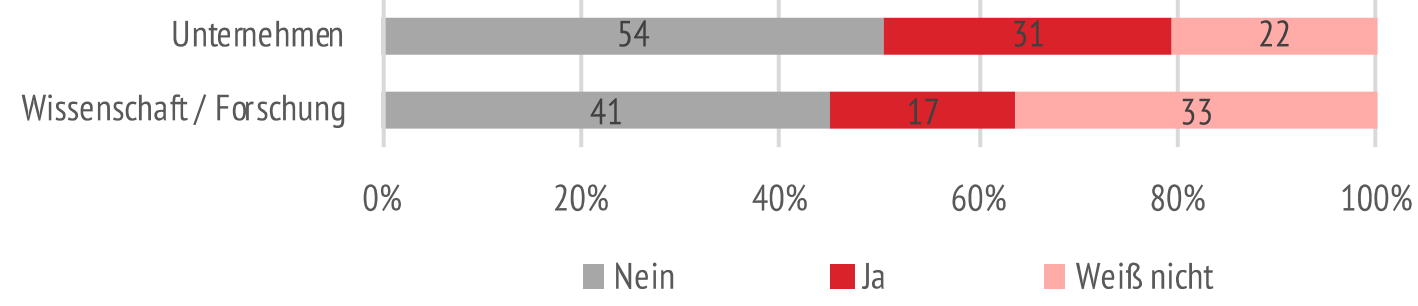

Quelle: Befragung Evaluierung der BMVIT IKT-Programme 2017; Auswertung inspire research

Jene Antwortenden, die an der thematischen Vorbereitung von Ausschreibungen des IKT der Zukunft-Programms beteiligt waren, schätzen die Auswirkungen ihrer Teilnahme als gut ein. Insgesamt 24 von 26 Unternehmensvertreter/inne/n gaben an, die Beteiligung an der inhaltlichen Vorbereitung der Ausschreibungen hätte dazu geführt, dass für ihre Einrichtung wesentlichen Themenschwerpunkte in den Ausschreibungen berücksichtigt wurden. Von insgesamt 16 beteiligten Vertreter/inne/n von Forschungseinrichtungen waren 13 der Ansicht, ihre Beteiligung hätte in relevantem oder sehr großem Ausmaß die thematische Ausrichtung der Ausschreibungen beeinflusst. 
Abbildung 24 In welchem Ausmaß konnte durch die Beteiligung an der inhaltlichen Vorbereitung der Ausschreibungen die für Ihre Einrichtung wesentlichen Themenschwerpunkte in der Ausschreibung berücksichtigt werden? Anzahl der Antworten nach Teilnehmergruppen

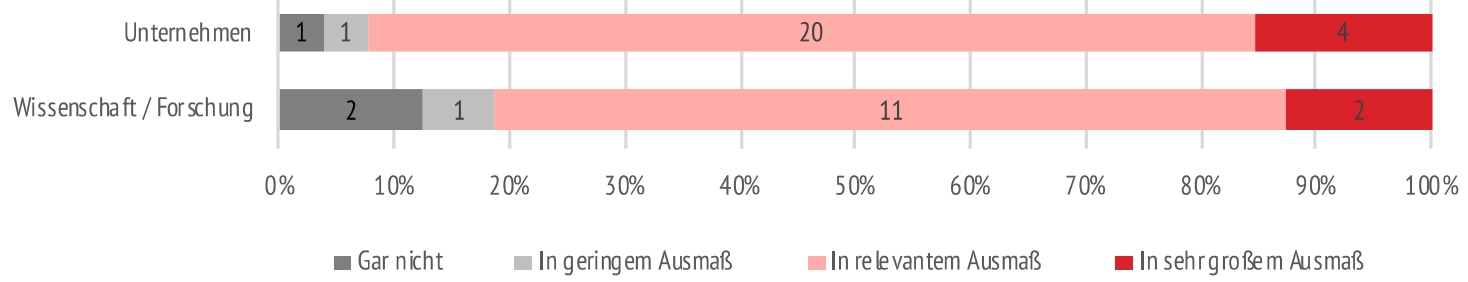

Quelle: Befragung Evaluierung der BMVIT IKT-Programme 2017; Auswertung inspire research

Unternehmen schätzen vor allem bei den Förderthemen Gemeinsame Technologieinitiative und Demographischer Wandel die eigenen Gestaltungs- und Einflussmöglichkeiten auf die Inhalte der Ausschreibungen hoch ein, während die Vertreter/innen der Forschungseinrichtungen größere Einflussmöglichkeiten in den nationalen Ausschreibungen der Themeninitiative IKT der Zukunft sehen.

\subsection{Einschätzung der Entwicklung des Programmumfelds}

Wie haben sich nach Einschätzung der Förderempfänger von FIT-IT Projekten die Rahmenbedingungen für IKT-bezogene Forschung und Entwicklung seit Abschluss ihrer Projekte insgesamt verändert? Falls ja, in welcher Art und Weise?

Fast zwei Drittel (64 \%) der Unternehmen und drei Viertel (74\%) der Forschungseinrichtungen gaben an, dass sich die fachliche Expertise der wissenschaftlichen Einrichtungen bei der FuEZusammenarbeit „etwas verbessert" oder "stark verbessert" hätte. Die Möglichkeiten der Zusammenarbeit mit wissenschaftlichen Einrichtungen hat sich für mehr als die Hälfte (55\%) der an FIT-IT beteiligten Unternehmen verbessert. Gleichzeitig nehmen auch die Forschungsreinrichtungen (58\%) eine Verbesserung der Möglichkeiten der Zusammenarbeit mit Unternehmen war, wobei sich aus Sicht der Mehrheit der Forschungseinrichtungen (53\%) auch die Mitarbeit der Unternehmen in FuE-Projekten nachhaltig verbessert hätte.

Abbildung 25 Wie haben sich für Ihre Einrichtung die Rahmenbedingungen für IKT-bezogene FuE seit Abschluss des Projekts entwickelt? Anteil der Antwortenden, die angaben, die folgenden Aspekte hätten sich "etwas verbessert" oder "stark verbessert", nach Teilnehmergruppen

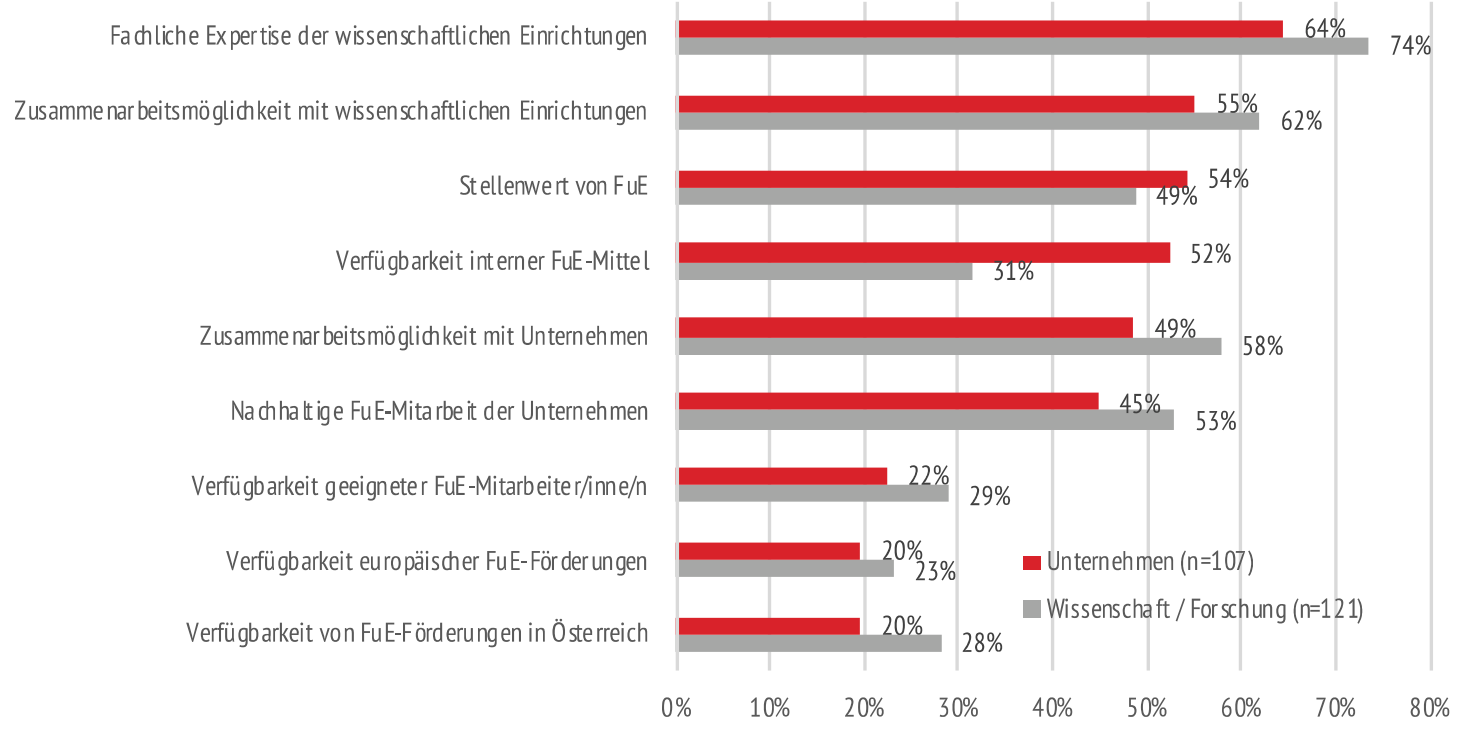

Quelle: Befragung Evaluierung BMVIT-IKT-Programme 2017; inspire research 
Kritisch wird hingegen weiterhin die Personalsituation gesehen. Nur $22 \%$ der Unternehmen und $29 \%$ der Forschungseinrichtungen gaben an, dass sich die Verfügbarkeit geeigneter FuE-Mitarbeiter/innen seit Abschluss der FIT-IT Projekte etwas oder stark verbessert hätte.

Auch bei der Verfügbarkeit von FuE-Förderungen in Österreich und in Europa sieht nur ein geringer Anteil der antwortenden Programmteilnehmer/inne/n Verbesserungen im Vergleich zum Zeitpunkt des Abschlusses des FIT-IT Projekts. Demgegenüber steht jedoch ein insgesamt höherer Stellenwert von FuE und eine bessere Verfügbarkeit interner FuE-Mittel in den Unternehmen.

Tabelle 26 Wie haben sich für Ihre Einrichtung die Rahmenbedingungen für IKT-bezogene FuE seit Abschluss des Projekts entwickelt? Anteil der Antwortenden, die angaben, die folgenden Aspekte hätten sich „etwas verbessert" oder „stark verbessert", nach Programmen und Teilnehmergruppen

\begin{tabular}{|c|c|c|c|c|c|c|}
\hline & \multicolumn{2}{|c|}{ FIT-IT } & \multicolumn{2}{|c|}{ GTI } & \multicolumn{2}{|c|}{ Dem. Wandel } \\
\hline & $\begin{array}{l}\text { Wissen- } \\
\text { schaft } \\
(\mathrm{N}=94)\end{array}$ & $\begin{array}{l}\text { Unter- } \\
\text { nehmen } \\
(\mathrm{N}=72)\end{array}$ & $\begin{array}{c}\text { Wissen- } \\
\text { schaft } \\
(\mathrm{N}=8)\end{array}$ & $\begin{array}{l}\text { Unter- } \\
\text { nehmen } \\
(\mathrm{N}=17)\end{array}$ & $\begin{array}{l}\text { Wissen- } \\
\text { schaft } \\
(\mathrm{N}=19)\end{array}$ & $\begin{array}{l}\text { Unter- } \\
\text { nehmen } \\
(\mathbf{N}=19)\end{array}$ \\
\hline Stellenwert von FuE & $50 \%$ & $61 \%$ & $38 \%$ & $53 \%$ & $47 \%$ & $26 \%$ \\
\hline Verfügbarkeit interner FuE-Mittel & $31 \%$ & $60 \%$ & $38 \%$ & $41 \%$ & $32 \%$ & $32 \%$ \\
\hline $\begin{array}{l}\text { Zusammenarbeitsmöglichkeit mit wissen- } \\
\text { schaftlichen Einrichtungen }\end{array}$ & $59 \%$ & $57 \%$ & $100 \%$ & $65 \%$ & $63 \%$ & $37 \%$ \\
\hline $\begin{array}{l}\text { Zusammenarbeitsmöglichkeit mit Unter- } \\
\text { nehmen }\end{array}$ & $54 \%$ & $53 \%$ & $75 \%$ & $53 \%$ & $68 \%$ & $26 \%$ \\
\hline $\begin{array}{l}\text { Fachliche Expertise der wissenschaftli- } \\
\text { chen Einrichtungen }\end{array}$ & $74 \%$ & $69 \%$ & $75 \%$ & $59 \%$ & $68 \%$ & $47 \%$ \\
\hline Nachhaltige FuE-Mitarbeit der Untern. & $52 \%$ & $47 \%$ & $75 \%$ & $47 \%$ & $47 \%$ & $32 \%$ \\
\hline Verfügbarkeit von FuE-Förd. in Österreich & $29 \%$ & $22 \%$ & $38 \%$ & $6 \%$ & $21 \%$ & $21 \%$ \\
\hline Verfügbarkeit europ. FuE-Förderungen & $21 \%$ & $21 \%$ & $38 \%$ & $12 \%$ & $26 \%$ & $21 \%$ \\
\hline $\begin{array}{l}\text { Verfügbarkeit geeigneter FuE-Mitarbei- } \\
\text { ter/inne/n }\end{array}$ & $27 \%$ & $26 \%$ & $38 \%$ & $18 \%$ & $37 \%$ & $11 \%$ \\
\hline
\end{tabular}

Quelle: Befragung Evaluierung der BMVIT IKT-Programme 2017; Auswertung inspire research

Beim Vergleich der Antworten der Projektteilnehmer/innen zwischen den drei Förderschwerpunkten fällt auf, dass die Unternehmen in Projekten zum Demographischen Wandel deutlich weniger häufig Verbesserungen beim Stellenwert von FuE, der Verfügbarkeit interner Mittel für FuE und den Zusammenarbeitsmöglichkeiten mit wissenschaftlichen Einrichtungen und Unternehmen sehen, als Unternehmen und Forschungseinrichtungen in anderen Programmen. Die beteiligten Forschungseinrichtungen sehen hingegen in allen drei Programmen in ähnlichem Ausmaß Verbesserungen diese Aspekte betreffend.

Außerdem fällt auf, dass es gerade Unternehmen in Projekten der Gemeinsamen Technologieinitiativen sind, die nur in sehr geringem Ausmaß Verbesserungen in der Verfügbarkeit von öffentlichen FuE-Förderungen sehen. Die Entwicklung der Förderdaten, die FuE-Statistik sowie die Kennzahlen zur Entwicklung des IKT-Sektors in Österreich (siehe Anhang) zeichnen hier interessanter Weise ein anderes Bild. 


\section{Hinweise zum Programmkonzept und zu den Förder- instrumenten}

In diesem Abschnitt fassen wir weitere Hinweise der Programmteilnehmer/innen zu einzelnen Aspekte der Themeninitiative IKT der Zukunft aus den Gesprächen und aus den schriftlichen Rückmeldungen der Förderempfänger/innen in den Befragungen zusammen.

\subsection{Inhaltliche Ausrichtung des Programms}

Bezüglich der thematischen Ausrichtung der Ausschreibungen wurde darauf hingewiesen, dass die IKT Branche sehr schnelllebig und zunehmend international orientiert sei. Eine zu restriktive thematische Fokussierung der Ausschreibungen biete daher oft nicht die Möglichkeit auf aktuelle Entwicklungen einzugehen. Das Bedienen von vermeintlichen Trends sei wenig zielführend und vor allem für Forschungseinrichtungen wenig attraktiv. Ein Teil der Programmteilnehmer/innen wünschten sich daher wieder mehr thematische Freiheiten in den Ausschreibungen mit der Möglichkeit, auch thematisch offene Anträge einzureichen. Angeregt wurde beispielsweise eine offene Förderschiene zum Thema Digitalisierung, mehr Interdisziplinarität in den Anwendungsfeldern bzw. generell die Steigerung der Diversität der thematischen Ausschreibungen.

Kritisch wurde von einigen Programmteilnehmer/inne/n die Verschiebung des Schwerpunkts der IKT-Förderung von Hardware (im Impulsprogramm FIT-IT) hin zu Software und Systemen (in der Themeninitiative IKT der Zukunft) wahrgenommen. Während für FuE mit Fokus auf Software und Systeme auch zahlreiche andere Förderprogramme bestehen würden, wären vor allem für grundlagennahe, hardwareorientierte Forschung alternative Förderungen schwer zu finden. Hardwareorientierte Projekte hätten in den aktuell bestehenden Programmschwerpunkten schlechte Chancen, gefördert zu werden.

Einige Förderempfänger gaben an, einen thematischen Fokus statt vorgegebener Anwendungsbereiche in den Ausschreibungen angemessener zu finden. Einzelne Förderempfänger/innen fanden hingegen die Programmschwerpunkte nach wie vor zu wenig anwendungsnahe. Projekte, die beispielsweise die Weiterentwicklung von Produktionstechnik behandeln bzw. neue Fertigungsverfahren implementieren würden, hätten derzeit kaum Chancen, gefördert zu werden. Es fehle eine Säule für industrielle Entwicklung. Andere meinten hingegen, dass die Themenfelder der Themeninitiative IKT der Zukunft ohnehin bereits sehr breit aufgestellt seien.

Einige Förderempfänger sprachen sich für die Einrichtung von Begleitgruppen für die geförderten Projekte in den nationalen Ausschreibungen von IKT der Zukunft aus. Außerdem regten einige Programmteilnehmer/innen an, das BMVIT bzw. die FFG sollten mehr Information über bereits laufende und abgeschlossene Forschungsaktivitäten der Themeninitiative IKT der Zukunft zur Verfügung stellen.

\subsection{Antragsverfahren und Berichtswesen}

Die Rückmeldungen von Programmteilnehmer/innen von IKT der Zukunft bestätigten die insgesamt hohe Qualität der Programmdurchführung. Das Ausschreibungsverfahren und die Projektabwicklung seien sehr gut, so der überwiegende Tenor der Rückmeldungen. Die Themeninitiative IKT der Zukunft könnte - wie bereits auch das Impulsprogramm FIT-IT - als Benchmark für andere (thematische) Programme der FFG dienen. 
Als besonders positiv erwähnten einige Antragsteller/innen die insgesamt gestiegene Qualität und Unterstützung der Antragstellung und Projektabwicklung durch das FFG eCall-System. Das Schema der Themeneinordung in den Ausschreibungsunterlagen könnte jedoch noch besser erläutert werden. Auf administrativer Seite wurde von mehreren Förderempfänger/innen der Aufwand für die Stundenabrechnungen kritisiert und vorgeschlagen, stärker mit Pauschalen oder Globalbudgets zu arbeiten. Auch sollten frühere Projekte oder Teile aus früheren Projekten einfacher fortgeführt werden können. Die Möglichkeit der Einbindung internationaler Partner/innen in die nationalen Projekte sollte weiter bestehen bleiben bzw. unterstützt werden.

Einige wissenschaftliche Projektpartner/innen schlugen vor, das Antragsverfahren durchgehend in Englisch durchzuführen bzw. die inhaltlichen Ausschreibungsunterlagen auch in Englisch zur Verfügung zu stellen. Ein Wissenschaftler gab beispielweise an, an seiner Abteilung habe ein Viertel der wissenschaftlichen Mitarbeiter/innen Deutsch nicht als Muttersprache. Diese Wissenschaftler/innen könnten sich an deutschsprachigen Ausschreibungen nicht beteiligen. Im sonstigen wissenschaftlichen Umfeld würden Deutschkenntnisse jedoch nicht gefordert oder benötigt werden.

Insgesamt betonten die Teilnehmer/innen am Programm, dass IKT der Zukunft eine ausgezeichnete Basis für FuE-Aktivitäten in der IKT-Forschung sei. Die Verfahren und die Abwicklung seitens der FFG seien sehr gut. Die Ausschreibungen seien klar strukturiert und die Ansprechpartner/innen bei der FFG hilfsbereit und kompetent. Mehr Budget für die Themeninitiative IKT der Zukunft wäre angesichts der Erfolgsquoten allerdings wünschenswert.

\subsection{Förderquoten und Finanzierung}

Aufgrund der höheren Förderintensität bei Projekten der industriellen Forschung im Vergleich zu jenen der experimentellen Entwicklung haben die Antragsteller ein großes Interesse, ihre Projekte als industrielle Forschung zu klassifizieren. Unternehmen streben in öffentlich geförderten kooperativen FuE-Projekten die Kategorie industrielle Forschung auch an, weil sie ihre entwicklungs- bzw. marktnäheren Vorhaben aus strategischen bzw. verwertungstechnischen Überlegungen entweder alleine durchführen oder bei notwendigen Kooperationen mit wissenschaftlichen Einrichtungen ein Auftragsverhältnis bevorzugen.

Die Vertreter einzelner Unternehmen wiesen auf Fälle hin, in denen es zu einer Umklassifizierung von Projektanträgen im Begutachtungsverfahren von der Kategorie „industrielle Forschung" zur Kategorie „experimentelle Entwicklung" gekommen sei. Diese Praxis sei sehr kritisch. Damit verringere sich nicht nur der öffentliche Finanzierungsbeitrag für den unternehmensinternen FuE-Teil, sondern die Unternehmen seien zudem häufig mit Anfragen der wissenschaftlichen Partner konfrontiert, zur Ausfinanzierung der nun deutlich höheren, nicht durch die Förderung gedeckten Kosten bei den Forschungseinrichtungen beizutragen. Dies würde die weitere Projektplanung erschweren und zusätzliche Abstimmungsprozesse im Konsortium notwendig machen.

Einige Unternehmensvertreter/innen gaben zwar an, ihre Unternehmen würden im Einzelfall Finanzierungsbeiträge zu den Projektkosten der wissenschaftlichen Partner leisten können, insbesondere wenn dies in den Kooperationsverträgen bereits vorgesehen sei. Andere Unternehmen stehen jedoch auf dem Standpunkt, dass eine Finanzierung von FuE-Arbeiten bei wissenschaftlichen Partnern nur bei entsprechendem Leistungsaustausch erfolgen sollte. Ein gemeinsames kooperatives FuE-Projekt ist jedoch keine FuE-Dienstleistung. Die Möglichkeiten der Querfinanzierung aus sonstigen Eigenmitteln ist insbesondere bei Hochschulen beschränkt. Sowohl Unternehmen als auch Forschungseinrichtungen sprachen sich daher in 
diesem Zusammenhang für realistischere Finanzierungsmodelle für die FuE-Anteile der Projekte aus, die von den wissenschaftlichen Partnern durchgeführt werden.

Wissenschaftliche Partner gaben zum Umfang von IKT der Zukunft-Projekte an, dieser sei häufig zu gering, um Doktoratsstudierenden eine Promotion zu ermöglichen. Man könne mit den Projekten meist nur Teile von Promotionen finanzieren. Dies führe zu Unsicherheit bei den Doktoratsstudierenden und habe in der Vergangenheit bereits qualifizierte Studierende davon abgehalten, ein IKT der Zukunft-gefördertes Promotionsthema anzunehmen. Eine Anpassung der Förderbedingungen an die Anforderungen einer Promotion würde helfen, mehr Doktorand/inn/en zu industriell relevanten Fragestellungen ausbilden zu können. Ein Gesprächspartner wies in diesem Zusammenhang auf das Instrument Leitprojekte hin, das ein geeignetes Vehikel sein könnte, verstärkt auch wieder Doktorand/inn/en in die Forschung einzubinden. Zeitlich und finanziell zu klein dimensionierte Vorhaben seien jedenfalls nicht dazu geeignet Doktorand/inn/en zu beschäftigen.

\subsection{Leitprojekte}

In der 4. Ausschreibung von IKT der Zukunft 2014 wurde erstmals ein Leitprojekt ausgeschrieben. Mit Leitprojekten sollen modellhafte Lösungen für bedeutende gesellschaftliche Herausforderungen bzw. integrierte Lösungen auf Systemebene entwickelt werden. Sie sollen die Wettbewerbsfähigkeit eines oder mehrerer Wirtschaftszweige unter Berücksichtigung der horizontalen bzw. vertikalen Integration in der Wertschöpfungskette stärken und eine langfristige Wachstumsperspektive für Technologien, Produkte, Verfahren und Dienstleistungen schaffen. Mit Leitprojekten soll die Sichtbarkeit österreichischer Technologien, Verfahren, Produkte und Dienstleistungen auf nationaler und internationaler Ebene sowie das Bewusstseins zum Nutzen der Lösungen in der Öffentlichkeit erhöht werden.

Leitprojekte sind mit einer Mindestfördersumme von 2 Mio. Euro deutlich größer dimensioniert als kooperative FuE-Projekte. Die durchschnittlich beantragten Kosten der kooperativen FuE-Projekte in der der 4. Ausschreibung von IKT der Zukunft betrugen rund 790 Tsd. Euro. Im einzigen beantragten (und dann auch geförderten Leitprojekt der Ausschreibung) waren Kosten in der Höhe von 3,83 Mio. Euro beantragt. In der zweiten Ausschreibung für Leitprojekte im Jahr 2016 wurden drei Anträge mit durchschnittlich beantragten Projektkosten von 4,64 Mio. Euro eingereicht, während in der vorangegangenen 5. Ausschreibung von IKT der Zukunft die durchschnittlich beantragten Projektkosten für kooperative FuE-Projekte bei 643 Tsd. Euro lagen. Leitprojekte haben damit etwa das fünf- bis siebenfache Projektbudget wie kooperative FuE-Projekte.

Dieser Größenunterschied spiegelt sich auch in der Anzahl der beteiligten Partner wider. An den beiden bisher bewilligten Leitprojekten sind jeweils 17 Partner als Förderempfänger beteiligt. Die durchschnittliche Anzahl der Förderempfänger in der 4. und 5. Ausschreibung von IKT der Zukunft lag bei rund 3.5 Projektpartnern. Die durchschnittlichen beantragten Projektkosten je Partner waren damit beim ersten bewilligten Leitprojekt vergleichbar hoch wie jene bei kooperativen FuE-Projekten, beim erfolgreichen Antrag der Leitprojektausschreibung 2016 mit ca. 300 Tsd. Euro beantragte Kosten je Partner um ca. 50 \% höher als der Durchschnitt der beantragten Kosten je Partner in den kooperativen FuE-Projekten der 5. Ausschreibung von IKT der Zukunft (ca. 190 Tsd. Euro).

Der Unternehmensanteil an den Gesamtkosten entsprach beim geförderten Leitprojekt 2014 mit rund $47 \%$ in etwa jenem in kooperativen FuE-Projekten der IKT der Zukunft-Ausschreibung 2014. Im geförderten Leitprojekt der Ausschreibung 2016 lag der Unternehmensanteil 
an den beantragten Gesamtkosten mit 59 \% jedoch deutlich über dem Niveau in kooperativen FuE-Projekten der vorangegangenen 5. Ausschreibung von IKT der Zukunft.

Nur sofern spezifische Unternehmen oder sonstige Einrichtungen benötigt werden, um die Wertschöpfungsketten des Leitprojekts angemessen abbilden zu können, sprechen die Leitprojekte auch neue Partner an. Am Leitprojekt 2014 sind zwei Partner beteiligt, die erstmals einen Antrag im BMVIT IKT-Programm gestellt haben. Bei den Unternehmen, die an den drei Anträgen der Leitprojektausschreibung 2016 beteiligt waren, handelte es sich allerdings ausschließlich um Einrichtungen, die bereits zu einem früheren Zeitpunkt an einem Antrag im BMVIT IKT-Programm mitwirkten und auch gefördert wurden. Aufgrund der bisherigen Erfahrungen kann daher nicht davon ausgegangen werden, dass mit Leitprojekten generell neue oder andere Partner angesprochen werden. Die Rückmeldungen von fünf der insgesamt elf beteiligten Unternehmen und sonstigen Partner, die keine Hochschulen oder außeruniversitäre Forschungseinrichtungen sind, zeigen auch, dass zahlreiche Vorerfahrungen bei der Zusammenarbeit mit den Partnern bestehen.

Aufgrund der höheren Anzahl an beteiligten Partnern konzentriert sich die praktische Zusammenarbeit zwischen den Partnern im laufenden Projekt oft nur auf einige Kernpartner, wobei jedoch jeweils die Rolle der Partner über Umfang und Intensität der Kooperationen entscheidet. Ein beteiligtes Unternehmen am Leitprojekt 2014 berichtete beispielsweise, es würde nur mit beteiligten Kunden und Pilotanwendern intensiv zusammenarbeiten, nicht aber mit den Forschungseinrichtungen oder den Entwicklern unter den Unternehmen. Ein anderes Unternehmen gab hingegen an, nur mit den beteiligten Hochschulen bzw. Forschungseinrichtungen und Intermediären intensiv zu kooperieren, nicht aber mit anderen beteiligten Unternehmen. Zwei Einrichtungen beschrieben die Zusammenarbeit mit den Partnern im Projekt hingegen insgesamt als intensiv. Die Muster der Zusammenarbeit spiegeln hier auch den Projektplan wider, der mehrere parallele Pilotentwicklungen vorsieht, an denen unterschiedliche Partner beteiligt sind.

Trotz des aufwändigeren Antragsverfahrens und des höheren Abstimmungsbedarfs in einem größeren Konsortium, konnte bisher der Zeitraum zwischen Einreichschluss für die Projektanträge und dem Start des Leitprojekts ähnlich kurzgehalten werden, wie bei kooperativen FuE-Projekten. Beim Leitprojekt 2014 betrug dieser Zeitraum 208 Tage und bei den kooperativen FuE-Projekten der 4. Ausschreibung von IKT der Zukunft lagen durchschnittlich 193 Tage zwischen den beiden Terminen. Beim Leitprojekt 2016 wurde ein Zeitraum von 200 Tagen benötigt. Der Durchschnitt für kooperative FuE-Projekte der 5. Ausschreibung von IKT der Zukunft betrug 217 Tage. Auch mit der Beratung und Unterstützung durch die FFG sind die beteiligten Partner im Leitprojekt 2014 durchschnittlich gleich zufrieden gewesen, wie Partner in zeitgleich durchgeführten kooperativen FuE-Projekten des Programms.

\subsection{Förderschwerpunkt Demographischer Wandel}

Einige Projektpartner/innen in nationalen und internationalen Projekten zum Demographischen Wandel waren der Ansicht, dass in den Projekten ein noch stärkerer Fokus auf die spätere wirtschaftliche Umsetzbarkeit bzw. Vermarktung gelegt werden sollte. Ein verwertbares Produkt bzw. eine verwertbare Dienstleistung sollte in den FuE-Arbeiten im Vordergrund stehen. Dazu sollten Pilotkunden (wie Enduser-Organisationen) besser eingebunden und ein stärkerer Fokus auf die zugrunde liegenden Business Modelle gelegt werden.

Zur Ausrichtung der Ausschreibungen wurde zudem erwähnt, dass vor allem bei den benefitDemonstrationsprojekten der Schwerpunkt jedenfalls nicht (nur) auf Technologie gelegt wer- 
den sollte, sondern auf Nutzung und Umsetzung, damit die Lösungen tatsächlich bedarfsnahe und bedarfsgerecht aufgegriffen und umgesetzt werden können. Die interdisziplinäre Zusammenarbeit mit Pflege- und Gesundheitswissenschaften sollte dazu stärker eingefordert werden. In den Projekten sollten auch sozialwissenschaftliche Forschungsfragen mit Fokus auf die Anwender/innen besser integriert werden. Technologieentwicklung sollte verstärkt als Beitrag zur Verbesserung sozialer Strukturen zur Bewältigung des Demographischen Wandels verstanden werden.

Bezüglich des Antrags- und Berichtswesens wünschten sich einige Projektpartner /innen nur eine zentrale Stelle, an die berichtet werden muss - das heißt, entweder an die FFG oder an das AAL-Büro in Brüssel. Außerdem sollten die komplexen, länderspezifischen Regeln für die Zusammensetzung der Konsortien reduziert und die nationalen Anforderungen an eine Förderung (einschließlich der Förderquoten) vereinheitlicht werden. Weitere Länder sollten wieder verstärkt in das AAL-Programm eingebunden werden.

Hingewiesen wurde zudem von Förderempfängern, dass die Projektkoordinator/inn/en in den transnationalen AAL-Projekten keine ausreichenden Gestaltungs-, Steuerungs- und Sanktionsmöglichkeiten gegenüber Mitgliedern des Konsortiums hätten (bzw. um die Einhaltung von Vereinbarungen laut Fördervertrag. Projektplan und Konsortialvertrag einzufordern). Die Koordinator/inn/en seien häufig nur Mittelsmänner bzw. Mittelsfrauen zwischen den Partner/innen, dem AAL-Büro in Brüssel und den nationalen Forschungsstellen, jedoch ohne formale Kompetenzen, weil die Finanzierung und Mittelverteilung national erfolgt.

\subsection{Förderschwerpunkt Gemeinsame Technologieinitiative ECSEL}

Einzelne Projektpartner/innen vertraten die Ansicht, dass im Antragsverfahren die zulässigen Seitenzahlen für Project Outline und Full Project Proposal weiter verringert werden sollten, um den Aufwand für die Einreicher/innen zu reduzieren. Außerdem sollte die nationale und die europäische Berichterstattung zusammengelegt werden. Schließlich sei die Zeitschiene der Ausschreibungen mit einer Projekteinreichung im September schlecht gewählt, da damit die Hauptphase der Ausarbeitung eines Antrags in die Urlaubszeit fallen würde.

Zu den Review-Prozessen in ECSEL-Projekten erwähnte ein Programmteilnehmer, dass die technischen Expert/inn/en durch das übermittelte Material überfordert seien und keinen realistischen Einblick bekämen, wie es um die Projektzusammenarbeit im Konsortium tatsächlich stehe. Die Projektkoordinator/inn/en hätten jedenfalls praktisch kaum Möglichkeiten, Druck auf Projektpartner auszuüben, die bei der operativen Projektdurchführung ein distanziertes Verhältnis zu ihren Partner/inne/n im Konsortium an den Tag legen würden. In ECSEL-Projekten gäbe es immer wieder Projektpartner/innen, die ganz klar ihre Partikularinteressen voranstellen würden: Auf Seite der Industrieunternehmen würden sich viele auf ihre intern ohnehin laufenden Entwicklungsarbeiten konzentrieren; auf Seite der Wissenschaft gäbe es Partner/innen, die vor allem in sich geschlossene Dissertationen und Forschungsarbeiten durchführen würden, für die externe Kooperation ebenfalls zweitrangig sei. Derartige Projektdefizite könnten mit den vorhandenen Reviewprozessen nicht erkannt werden.

Andere Programmteilnehmer/innen vertraten die Ansicht, dass die nationalen Förderungen für Forschungseinrichtungen in ECSEL-Projekten dringend erhöht werden müssten, um international wettbewerbsfähig zu bleiben. Andere Länder würden ihre Forschungseinrichtungen mit höheren Budgets fördern und könnten damit Projekte auch eher beeinflussen. Auf Grund der hohen notwendigen Eigenleistungen der österreichischen Forschungseinrichtungen könnten diese nur mit vergleichsweise kleinen Budgets an Projekten mitwirken. ECSEL- 
Projekte seien für Forschungseinrichtungen jedoch strategisch wichtig, da diese die Verbindung zu Unternehmen im nationalen und internationalen Umfeld gewährleisten würden. Forschungseinrichtungen könnten sich in ECSEL-Projekten mit Unternehmen gut vernetzen, Probleme erkennen und gemeinsam Problemlösungen erarbeiten.

Seit der Zusammenführung von ARTEMIS und ENIAC zu ECSEL sei der Fokus des Programms unklarer und die beteiligten Industriezweige deutlich breiter geworden. Die Mitgestaltungsmöglichkeiten für Forschungseinrichtungen bezüglich Arbeitsprogramm hätten sich damit weiter verringert. Kritisiert wurde auch das als zu starr empfundene Korsett für die Einhaltung der Struktur des Kostenplans bzw. der Umwidmung von Kosten unterschiedlicher Kategorien. 


\section{$5 \quad$ Wirkungen der Förderung}

In diesem Abschnitt stellen wir die Angaben und Hinweise der Förderempfänger zu den Ergebnissen und Wirkungen der geförderten Projekte vor. Ergänzt werden die Informationen der Förderempfänger durch bibliometrische Auswertungen zu den Veröffentlichungen der technischen Ansprechpersonen der wissenschaftlichen Projektpartner und der Unternehmen und einer Analyse der Patentierungstätigkeit der an FIT-IT Projekten beteiligten Unternehmen. Außerdem präsentieren wir in diesem Abschnitt die Ergebnisse einer Sonderauswertung der FuE-Erhebungen von Statistik Austria, in der wir für geförderte und nicht geförderte Unternehmen die Entwicklung ihrer internen FuE-Ausgaben und der Anzahl der FuEBeschäftigten gegenüberstellen.

\subsection{Langfristige Nachwirkungen der FIT-IT-Projektförderung}

Für die Evaluierung befragten wir die technischen Ansprechpersonen der Förderempfänger des Impulsprogramms FIT-IT, ob das Projekt oder die erzielten Ergebnisse des Projekts über die Projektlaufzeit hinaus noch eine Nachwirkung in der Einrichtung hat bzw. in der Vergangenheit hatte.

Die Antworten zeigen, dass die wissenschaftlichen Einrichtungen (Hochschulen und außeruniversitäre Forschungseinrichtungen) Nachwirkungen der geförderten IKT-Projekte heute noch etwas häufiger wahrnehmen als die antwortenden Vertreter/innen der Unternehmen (einschließlich nicht-gewerblicher Unternehmen und sonstiger Partner, die nicht dem wissenschaftlichen Bereich zuzurechnen sind).

Abbildung 26 Hatte das Projekt bzw. hatten die erzielten Ergebnisse des Projekts über die Projektlaufzeit hinaus eine Nachwirkung in Ihrer Einrichtung? Anzahl der Antworten der Befragten nach Teilnehmergruppen und Programm

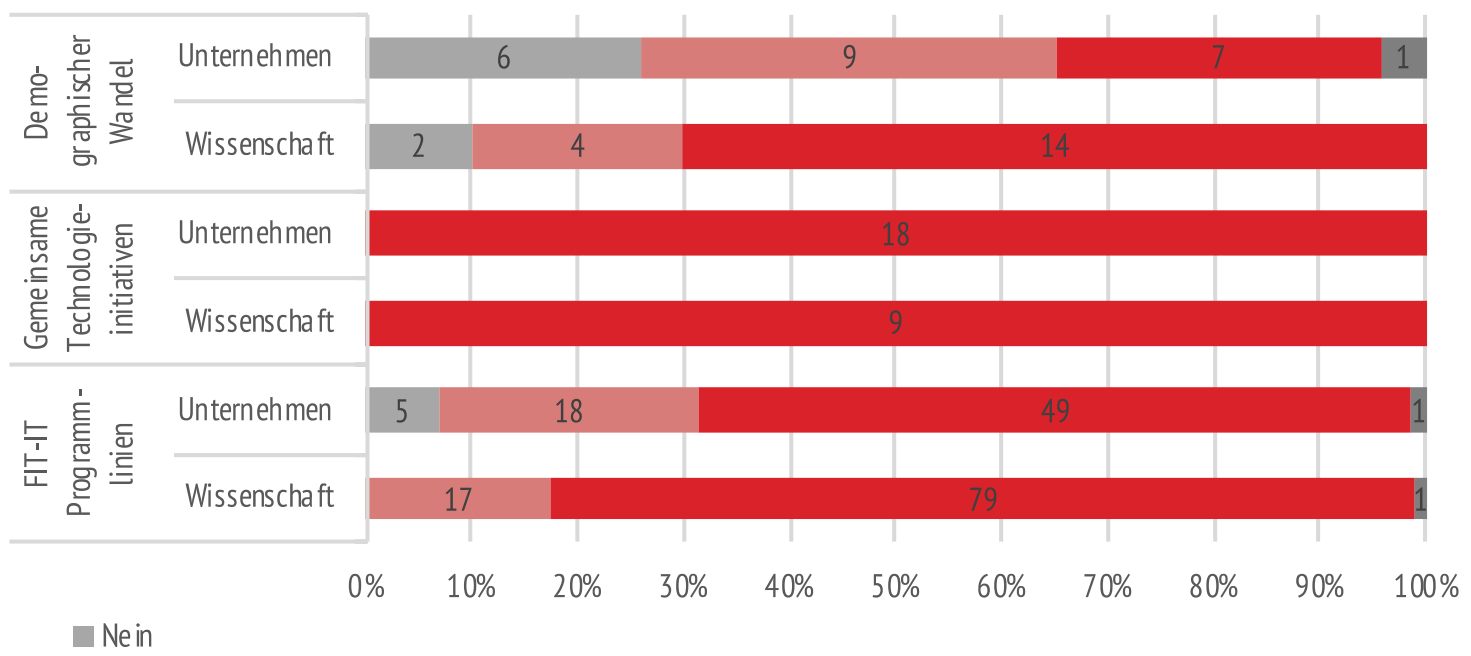

- Ja, das Projek thatte ein Nachwirkung über die Projekt laufzeit hinaus, die jedoch heute nicht mehr von Bede utung ist.

- Ja, das Projekthatte ein Nachwirkung über die Projekt laufzeit hinaus, die auch heute noch von Bedeutung ist.

Weiß nicht

Quelle: Befragung Evaluierung BMVIT-IKT-Programme 2017; inspire research

Auffällig beim Vergleich der Antworten zwischen den drei Programmen ist, dass alle antwortenden Vertreter/innen aus Wissenschaft und Unternehmen, die an ARTEMIS und ENIACProjekten beteiligt waren, auch heute noch eine Nachwirkung der Projekte in der eigenen 
Einrichtung wahrnehmen. Unter den beteiligten Einrichtungen an nationalen FIT-IT Projekten sind es immerhin noch mehr als zwei Drittel der Unternehmen und vier Fünftel der wissenschaftlichen Einrichtungen, die auch Jahre nach Abschluss des Projekts einen bleibenden Effekt der Förderung sehen. Am wenigsten häufig sehen die ehemaligen Beteiligten an nationalen und transnationalen Projekten zum Demographischen Wandel (Programm benefit, AAL) heute noch eine bleibende Nachwirkung der Projekte bzw. der Ergebnisse der Projekte in ihren Einrichtungen. Rund zwei Drittel der wissenschaftlichen Einrichtungen aber nur ein Drittel der beteiligten Unternehmen nimmt heute noch eine Nachwirkung des Projekts wahr.

\section{Gründe, warum FIT-IT Projekte heute keine Nachwirkungen mehr haben}

Als Gründe, warum die Projekte keine Nachwirkungen hatten oder diese heute nicht mehr von Bedeutung sind, wurden von den Antwortenden in Projekten zum Demographischen Wandel am häufigsten genannt, dass die Zusammenarbeit mit den Unternehmen und den wissenschaftlichen Partnern des Projekts inzwischen beendet worden sei. Es scheint den Projektteilnehmer/inne/ $\mathrm{n}$ in diesen Fällen nicht gelungen zu sein, langfristig stabile Kooperationsnetzwerke über die Dauer der Projekte hinaus zu etablieren. Auch wurde mehrfach erwähnt, dass die im Projekt erzielten Ergebnisse inzwischen bereits Stand der Technik seien. Einzelne entwickelte Lösungen seien außerdem zu aufwendig gewesen und wären durch die Endanwender/innen daher nicht angenommen worden.

Die Antwortenden, die an FIT-IT Projekten beteiligt waren, führten am häufigsten an, dass sich die am Projekt beteiligten Mitarbeiter/innen heute mit anderen Themen beschäftigen würden und das Projekt daher aus diesem Grund keine Nachwirkungen mehr habe. An zweiter Stelle unter den Gründen steht die Beendigung der Zusammenarbeit mit den Unternehmen des Projekts. Für die wissenschaftlichen Einrichtungen spielt auch der Weggang der am Projekt beteiligten Mitarbeiter/innen eine wesentliche Rolle, warum die Projekte heute keine Nachwirkungen mehr haben bzw. diese heute nicht mehr von Bedeutung sind.

Weitere genannte Gründe, warum die Projekte keine Nachwirkungen haben oder hatten, waren der Konkurs von beteiligten Unternehmen oder auch die im Projekt gewonnene Erkenntnis, dass mit der Verwertung der Ergebnisse ein zu großer finanzieller Aufwand verbunden gewesen wäre, der die Möglichkeiten der Unternehmen überstiegen hätte. Andere Unternehmen wiesen darauf hin, dass Marktrecherchen nach dem Projekt nur einen geringen Bedarf für die entwickelten Lösungen gezeigt hätten oder dass die Kosten für eine Entwicklung bis zur Marktreife zu hoch gewesen seien.

\section{Qualifizierung und Know-how-Aufbau}

Bei der Frage nach der Art der Nachwirkungen in den Einrichtungen über die Projektlaufzeit hinaus wurde am häufigsten der Beitrag der Projekte zur Qualifizierung der Mitarbeiter/innen sowie der Beitrag zum Know-how-Aufbau in der Einrichtung genannt. Zahlreiche Unternehmen gaben an, dass das vorhandene interne Know-how durch die Projekte deutlich verbessert worden sei. Das aufgebaute Know-how würde noch heute bei der Produktentwicklung und im Kundensupport helfen. Viele Unternehmen gaben auch an, wissenschaftliches Personal des Forschungspartners (z. B. Dissertant/inn/en, Master-Studierende) in den Betrieb übernommen zu haben. Die Unternehmen hätten nunmehr eigene Expert/inn/en im Haus.

Wissenschaftliche Einrichtungen berichteten, dass sie durch die FIT-IT-Förderung ihre Forschungsgruppen aufbauen hätten können, die auch hetue noch an den Themen arbeiten würden. Darüber hinaus seien zahlreiche Masterarbeiten, (Beiträge zu) Promotionen und Post-doc-Projekte entstanden. Außerdem seien die Ergebnisse in die Lehre eingeflossen 


\section{Box 1: Langfristige Wirkungen der FIT-IT Förderung: pureNFC (Performant Usable Reliable and Embedded NFC Systems)}

Das pureNFC-Projekt wurde in der 6. Ausschreibung der Programmlinie Embedded Systems eingereicht und zwischen 2007 und 2009 gefördert. Das Projekt wurde durch die FH Oberösterreich koordiniert. Als weiterer wissenschaftlicher Partner war die Technische Universität Wien am Projekt beteiligt. Als Unternehmenspartner arbeiteten NPX Semiconductors, Mobilkom Austria (heute A1 Telekom Austria) sowie Omnikey (heute Teil von HID Global) an den Forschungs- und Entwicklungsaufgaben mit.

Auf wissenschaftlicher Ebene konnten im Projekt zwei Dissertationen abgeschlossen werden. Diese wurden an der FH Oberösterreich fachlich durchgeführt und von der Johannes Kepler Universität (JKU) Linz betreut. Einer der ehemaligen Doktoranden hat inzwischen eine Postdoc-Position an der JKU Linz angenommen. Der zweite ehemalige Doktorand arbeitete einige Jahre in einem Spin-off Unternehmen des Projektpartners der TU Wien und leitet inzwischen für ein großes privates Medienhaus in Deutschland eine Abteilung, die sich mit Technologien der Zukunft für die Medienbereitstellung beschäftigt.

An der FH Oberösterreich war auch in den Folgejahren des Projekts NFC weiter eines der Kernforschungsthemen. Im Jahr 2009 fand der erste "International Workshop on Near Field Communication (NFC'Og)" am FH-Standort Hagenberg statt. In den Folgejahren wurde dieser "International Workshop on Near Field Communication" vom NFC Research Lab Hagenberg gemeinsam mit dem VTT Technical Research Centre of Finland und der ETH Zürich organisiert. Noch heute organisiert die FH Oberösterreich gemeinsam mit Seibersdorf Laboratories jährlich eine NFC-Tagung. Die wissenschaftliche Verwertung der Ergebnisse erfolgte über zahlreiche internationale Konferenzbeiträge. Alleine für den Projektleiter finden sich für den Zeitraum 2007 bis 2013 insgesamt 32 Scopus-Einträge zu wissenschaftlichen Publikationen mit Themenbezug NFC.

Aus Sicht des beteiligten Unternehmens NXP Semiconductors stellte pureNFC einen FuEBaustein dar, der zum großen Erfolg der NFC-Technologie am Standort Gratkorn beitrug. Inzwischen wurde NFC weltweit erfolgreich auf den Markt gebracht und ist auch für Endkunden in vielen Anwendungen greifbar, z. B. beim kontaktlosen Bezahlen. Ein Mitarbeiter des NXP Standorts Gratkorn, Franz Amtmann, erhielt im Jahr 2015 für die Erfindungen im Bereich NFC den europäischen Erfinderpreis. Die dem Preis zugrundeliegenden Patente wurden jedoch bereits einige Jahre vor Start des Projekts pureNFC angemeldet.

Nach Einschätzung von NXP hat die Förderung in den Pionierjahren von NFC wesentlich zur positiven Entwicklung des Standorts Gratkorn beigetragen. pureNFC habe wesentliche technische Aspekte gelöst, die einen positiven Einfluss auf die Usability von NFC-Systemen hatten und damit die spätere breite Marktakzeptanz sicherten.

Das Projekt war für NXP auch der Startpunkt für eine längerfristige Zusammenarbeit, sowohl mit der FH Oberösterreich, die in ein Josef Ressel-Labor mündete, als auch mit der TU Wien, an der später ein gemeinsames Christian Doppler-Labor eingerichtet wurde. Mit dem beteiligten Technologieunternehmen Omnikey gebe es nach wie vor Arbeitsbeziehungen. Die Kooperation mit dem Anwender Mobilkom (nunmehr A1 Telekom Austria) im Projekt habe hingegen zu keinen unmittelbaren Anwendungen in Österreich geführt. Die Zeit sei damals für Lösungen, die unbedingt internationale Standards und eine NFC-Anwendung in mobilen Endgeräten erforderte, noch nicht reif gewesen. Insgesamt habe das Projekt pureNFC aber deutlich positive Wirkungen sowohl auf technischer Ebene (z. B. Leistungsfähigkeit und Produktdifferenzierung) als auch für Kooperationen mit den wissenschaftlichen Partnern an österreichischen Hochschulen gehabt. 
und hätten zum Aufbau neuer Lehrinhalte in den Studiengängen geführt. Viele wissenschaftliche Partner erwähnten auch wissenschaftliche Publikationen aus den FIT-IT-Projekten, die nach wie vor zitiert werden würden.

\section{Folgeprojekte}

Auch Folgeprojekte wurden als Nachwirkung der geförderten FIT-IT Vorhaben sehr häufig genannt. Eine große Anzahl der Projektpartner gab an, die FIT-IT Projekte seien Ausgangspunkt für zahlreiche nationale und europäische Folgeprojekte gewesen. Als nationale Förderprogramme für diese Folgevorhaben wurden neben FIT-IT bzW. IKT der Zukunft vor allem andere thematische BMVIT-Programme (z. B. Produktion der Zukunft, KIRAS), das Kompetenzzentrenprogramm COMET und grundlagenforschungsnahe Programme des FWF, des WWTF und der Christian Doppler-Gesellschaft genannt. Bei aus europäischen Quellen geförderten Folgeprojekten wurden die EU-Forschungsrahmenprogramme RP7 und Horizon 2020 genannt, daneben aber auch Eurostars. Einige Projektpartner berichteten, die FIT-IT Projekte hätten eine große Bedeutung gehabt, um zu den Netzwerken der Gemeinsamen Technologieinitiativen ARTEMIS und ENIAC Zugang zu finden. Noch heute würden gemeinsame Projekte mit ehemaligen Partnern der FIT-IT Projekte in ECSEL-Projekten weiterlaufen.

Schließlich gaben Unternehmenspartner in der Befragung an, es seien in der Folge von FITIT Projekten kommerzielle Projekte mit Entwicklungspartnern und Kunden abgeschlossen worden. Die beteiligten Industriepartner hätten nach den FIT-IT Projekt gemeinsam die Weiterentwicklung der Ergebnisse vorangetrieben. Ergebnisse hätten Eingang in Produkte gefunden, die in zahlreichen Fällen auch erfolgreich wirtschaftlich vermarktet werden konnten. Einige Unternehmen berichteten über höhere Investitionen aufgrund der erzielten Ergebnisse in den FIT-IT Projekten.

Abbildung 27 Nachwirkungen der Projekte bzw. der Projektergebnisse über die Projektlaufzeit hinaus, nach Teilnehmergruppe

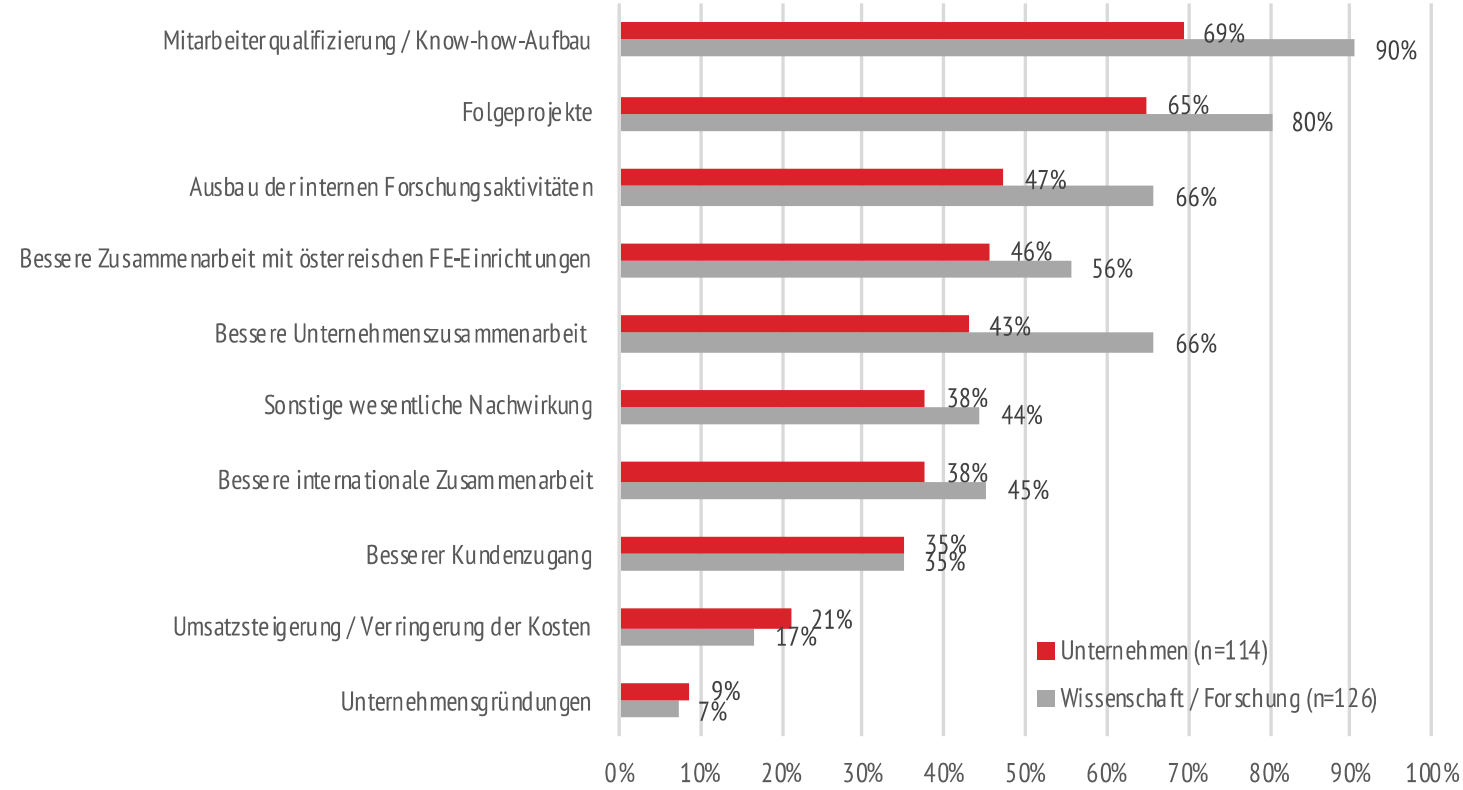

Quelle: Befragung Evaluierung BMVIT-IKT-Programme 2017; inspire research

Die Kontakte und der Austausch mit einzelnen Projektpartnern würden in vielen Unternehmen heute noch bestehen bzw. weiter fortgesetzt. Die Projekte hätten zu nachhaltigen Kooperationen zwischen den Projektpartnern geführt, mit jedenfalls positiven Folgeeffekten für 
die beteiligten Einrichtungen. Es gibt heute nach wie vor Unternehmen und Forschungseinrichtungen, die sich alleine oder in Kooperation mit (ehemaligen) Partnern mit der Optimierung der erzielten Lösungen in FIT-IT Projekten beschäftigen.

\section{Verbesserung der nationalen und internationalen Netzwerke und Zusammenarbeit}

Zahlreiche Vertreter/innen von Unternehmen und Forschungsreinrichtungen berichteten über eine deutlich verbesserte internationale Positionierung durch die Teilnahme an den Projekten. Dies trifft nicht nur auf die ohnehin transnational oder europäisch ausgerichteten Programmteile zu, sondern auch auf die nationalen FIT-IT Programmlinien. In diesen konnten vor allem die wissenschaftlichen Einrichtungen grundlagenorientierte FuE betreiben bzw. neue FuE-Themen vorbereiten, die später auch international sichtbar wurden bzw. in europäische Projekte mündeten.

Auch für die Sondierungsprojekte legen die Hinweise aus unseren Gesprächen und den Rückmeldungen der Teilnehmer/innen an der Befragung nahe, dass sie insgesamt erfolgreich waren bzw. Aufgangspunkt für zahlreiche Folgeprojekte. So berichtete ein Förderempfänger über ein FIT-IT finanziertes Sondierungsprojekt, in dem die Einreichung eines Projekts im EU-Forschungsrahmenprogramm vorbereitet wurde. Dieses Sondierungsprojekt habe substanziell dazu beigetragen, im EU-Verfahren einen sehr guten und erfolgreichen Antrag stellen zu können. Das österreichische Kernteam konnte in der Folge mit namhaften europäischen Partnern und Forschungseinrichtungen zusammenarbeiten. Mit den meisten Partnern wurde auch in den Folgejahren in europäischen Projekten kooperiert, wobei sich eine dauerhafte Community entwickelt habe. Gegenwärtig würden noch mit einigen der ursprünglichen Partner gemeinsame ECSEL-Projekte durchgeführt werden. Auch auf nationaler Ebene gebe es weiterhin eine erfolgreiche Zusammenarbeit mit den ehemaligen Partnern, wobei diese nicht auf IKT der Zukunft beschränkt sei, sondern auch in anderen BMVIT-Programmen stattfinde.

Tabelle 27 Nachwirkungen der Projekte bzw. der Projektergebnisse über die Projektlaufzeit hinaus, nach Teilnehmergruppe und Programm

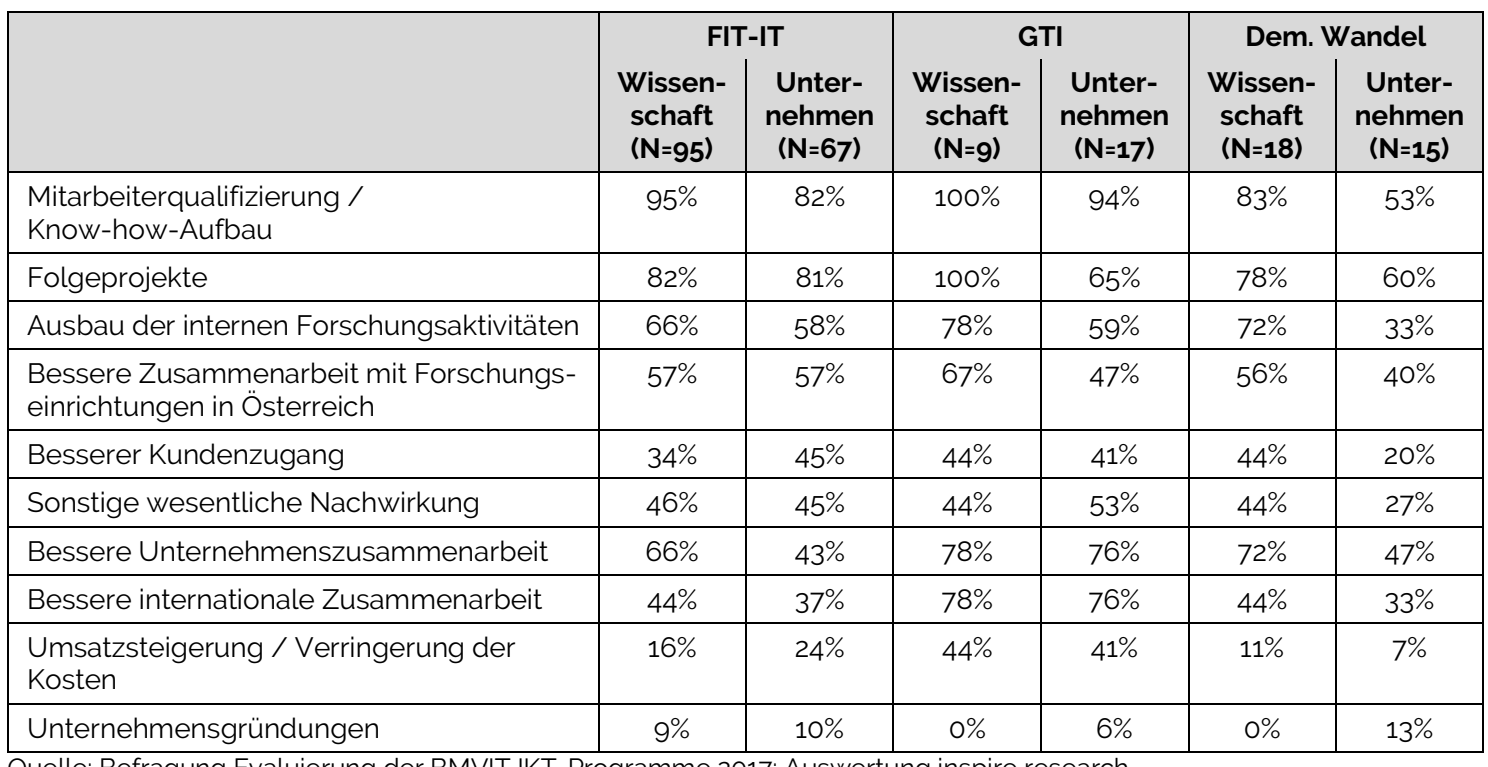

Positiv erwähnt betreffend Nachhaltigkeit der Förderung wurden auch ehemalige Begleitmaßnahmen von FIT-IT, wie beispielsweise die Unterstützung von Sommerschulen oder von wissenschaftlichen Konferenzen. Ehemals geförderte Sommerschulen würden nach wie vor 
stattfinden. Auch ehemals geförderte (internationale) Symposien würden nach wie vor abgehalten.

Nur in Einzelfällen wurde über Fälle berichtet, in denen das FIT-IT Projekt zu ausschließlich negativen Effekten geführt habe, vor allem aufgrund von Konkursen von Partnern oder geänderter Prioritäten im eigenen Unternehmen. Technisches Scheitern alleine wird von vielen Projektteilnehmer/inne/ $\mathrm{n}$ aber nicht als nur negativer Effekt der Projektteilnahme bewertet. So gaben Förderempfänger/innen an, es seien gerade die Schwierigkeiten im Projekt gewesen, die später für die Weiterentwicklung von Produkten und Dienstleistungen besonders hilfreich waren.

\section{Unterschiede in den Auswirkungen zwischen den Förderschwerpunkten}

Auffällig an den Befragungsergebnissen ist, dass Unternehmen (und andere nicht-wissenschaftliche Partner) in Projekten zum Demographischen Wandel anteilsmäßig den Ausbau der internen Forschungsaktivitäten weniger häufig als Nachwirkung der Projekte benennen. In Projekten zum Demographischen Wandel nehmen die beteiligten Unternehmen auch weniger häufig Nachwirkungen im Sinne eines besseren Zugangs zu Kunden sowie eines Beitrags zur Steigerung des Umsatzes bzw. Reduzierung der Kosten wahr. Projekte zum Demographischen Wandel dürften trotz des Programmanspruchs, vergleichsweise nahe am Markt nach neuen IKT-basierten Lösungen für die Herausforderungen der alternden Gesellschaft zu forschen, aufgrund der deutlich komplexeren Partner- und Marktstrukturen an der tatsächlichen Verwertung bzw. Nutzbarmachung der Ergebnisse häufiger gescheitert sein, als FuE-Projekte, die klassisch zwischen forschungsaktiven Unternehmen und ihren wissenschaftlichen Partnern durchgeführt werden.

\subsection{Nutzen und Auswirkungen von IKT der Zukunft-Projekten}

Die technischen Ansprechpersonen der Projekte der Themeninitiative IKT der Zukunft wurden ebenfalls in einer Online-Befragung nach den bereits sichtbaren Auswirkungen der Durchführung der Projekte befragt. Dabei ist zu beachten, dass nur ein Teil der Befragten zu dieser Frage bereits Antworten geben konnten, da in vielen Fällen die geförderten Projekte noch nicht abgeschlossen sind.

Die Ergebnisse zeigen, dass fast drei Viertel (73\%) der an den Projekten beteiligten Unternehmen die erzielten Lösungen in den Projekten intern weiterentwickeln. Etwas mehr als ein Drittel der Unternehmen (36\%) gibt an, es seien bereits eigene Produkte, Verfahren oder Dienstleistungen am Markt, die im Zusammenhang mit der Durchführung der Projekte entwickelt bzw. verbessert wurden. Die Verwertung über Patente spielt hingegen bisher nur eine untergeordnete Rolle. Insgesamt 7 \% der Unternehmen gaben an, Projektergebnisse bereits selbst patentiert zu haben. Betrachten wir nur die Unternehmen, die ihr Projekt bereits bis Ende 2016 abgeschlossen hatten, liegt dieser Anteil jedoch bei $17 \%$.

Bei den Universitäten und außeruniversitären Forschungsreinrichtungen dominiert unter den Ergebnissen der Projekte die wissenschaftliche Verwertung. Mehr als drei Viertel (77\%) der wissenschaftlichen Partner gab ab. Projektergebnisse bereits selbst wissenschaftlich publiziert zu haben. Fast zwei Drittel (62\%) der wissenschaftlichen Einrichtungen führen die Entwicklung von Prototypen und / oder Pilotanwendungen auch weiter fort. 


\section{Box 2: Langfristige Wirkungen der FIT-IT Förderung: DYONIPOS (Dynamic Ontology based Integrated Process Optimisation)}

Das Projekt DYONIPOS wurde in den Jahren 2006 bis 2008 als einer der erfolgreichen Anträge in der 2. Ausschreibung der FIT-IT Programmlinie Semantic Systems gefördert. Koordiniert wurde das Projekt vom Kompetenzzentrum Know-Center Graz. Der damalige Koordinator des Projekts ist seit 2012 Professor für Medieninformatik an der Universität Passau. Als weiterer wissenschaftlicher Partner war das Institut für Informationssysteme und Computermedien der TU Graz an DYONIPOS beteiligt. Unternehmenspartner des Projekts waren Hewlett-Packard Austria und m2n. Das Projekt entwickelte Lösungen zur flexiblen Organisation von Geschäftsprozessen mittels Wissensflussanalyse. Die Idee zum Projekt wurde gemeinsam zwischen dem Know-Center Graz, man und HP entwickelt. Die TU Graz ergänzte das Team mit Expertise zur Visualisierung von Prozessen. Die Beiträge der wissenschaftlichen Partner und der beteiligten Unternehmen im Projekt waren eng miteinander verzahnt, was eine enge Kooperation und regelmäßigen Austausch erforderte.

Nach Einschätzung der Projektbeteiligten haben es DYONIPOS-Projektergebnisse in einem vergleichsweise kurzen Zeitraum in die praktische Anwendung geschafft. Dies sei gelungen, weil parallel zu DYONIPOS weitere unterstützende Vorhaben realisiert werden konnten. Ganz zentral sei ein begleitendes Use case-Projekt mit dem Bundesministerium für Finanzen gewesen. Als Ergebnis des DYONIPOS Projekts und des DYONIPOS Use caseProjekts konnten im Finanzministerium schließlich drei Applikationen verwirklich werden:

Erstens baute ein bis 2014 intern eingesetzter Bürgerservice-Assistent auf DYONIPOS-Ergebnissen auf. Der Assistent unterstützte die Service-Mitarbeiter/innen bei der Bearbeitung von Bürgeranfragen. Eine zweite Applikation wurde für die strategische Kommunikationsanalyse entwickelt, mit der auch externe Datenquellen eingebunden werden konnten. Eine dritte, heute noch relevante Anwendung betrifft das Analysesystem, das vom Bundesministerium für Finanzen für die Betrugserkennung und Betrugsbekämpfung verwendet wird. Die Applikation erlaubt der Steuerfahndung das effiziente Suchen und Filtern von analogen und digitalen Daten aus heterogenen Informationsquellen.

Für den langfristigen Nutzen der erzielten Ergebnisse im Projekt DYONIPOS war daher das für Forschung und Forschungskooperationen sehr offene Team in der zuständigen Abteilung im Finanzministerium ganz wesentlich. Die BMF-Beteiligten verstanden ihre Rolle im Projekt nicht als passive Anwender von Forschungsergebnissen, sondern beteiligten sich aktiv am Forschungsprozess durch Rückkoppeln der eigenen internen Entwicklungsarbeiten in die wissenschaftliche Diskussion. Es existieren zahlreiche Beiträge der am Projekt beteiligen BMF-Mitarbeiter/innen zu DYONIPOS, die auf wissenschaftlichen Konferenzen präsentiert und diskutiert wurden.

Für das Unternehmen man haben sich aus dem Projekt zahlreiche nationale und europäische Folgeprojekte ergeben. Dazu kommen Umsetzungsprojekte mit österreichischen Ministerien. Die Kooperationen, die mit DYONIPOS gestartet wurden, bestehen auch heute noch. Zum Projektpartner Hewlett-Packard gibt es hingegen keine Arbeitskontakte mehr die auf DYONIPOS zurückgehen. Grund dafür war die Strategieentscheidung von HewlettPackard, die DYONIPOS Entwicklungen nicht weiterzuverfolgen.

Nur beschränkt erfolgreich war das Projekt in Bezug auf die Ausbildung des wissenschaftlichen Nachwuchses. Zwar beteiligten sich Doktoranden am Know-Center Graz an der Durchführung der DYONISOS Forschungsarbeiten. Die Projektlaufzeit von zwei Jahren reichte jedoch nicht aus, damit die Kandidaten ihre Promotionen hätten abschließen können. 
Abbildung 28 Unmittelbare wirtschaftliche oder wissenschaftliche Auswirkungen der Durchführung des Projekts: Anteil der Antwortenden, die angaben, folgende Auswirkungen seien bereits sichtbar

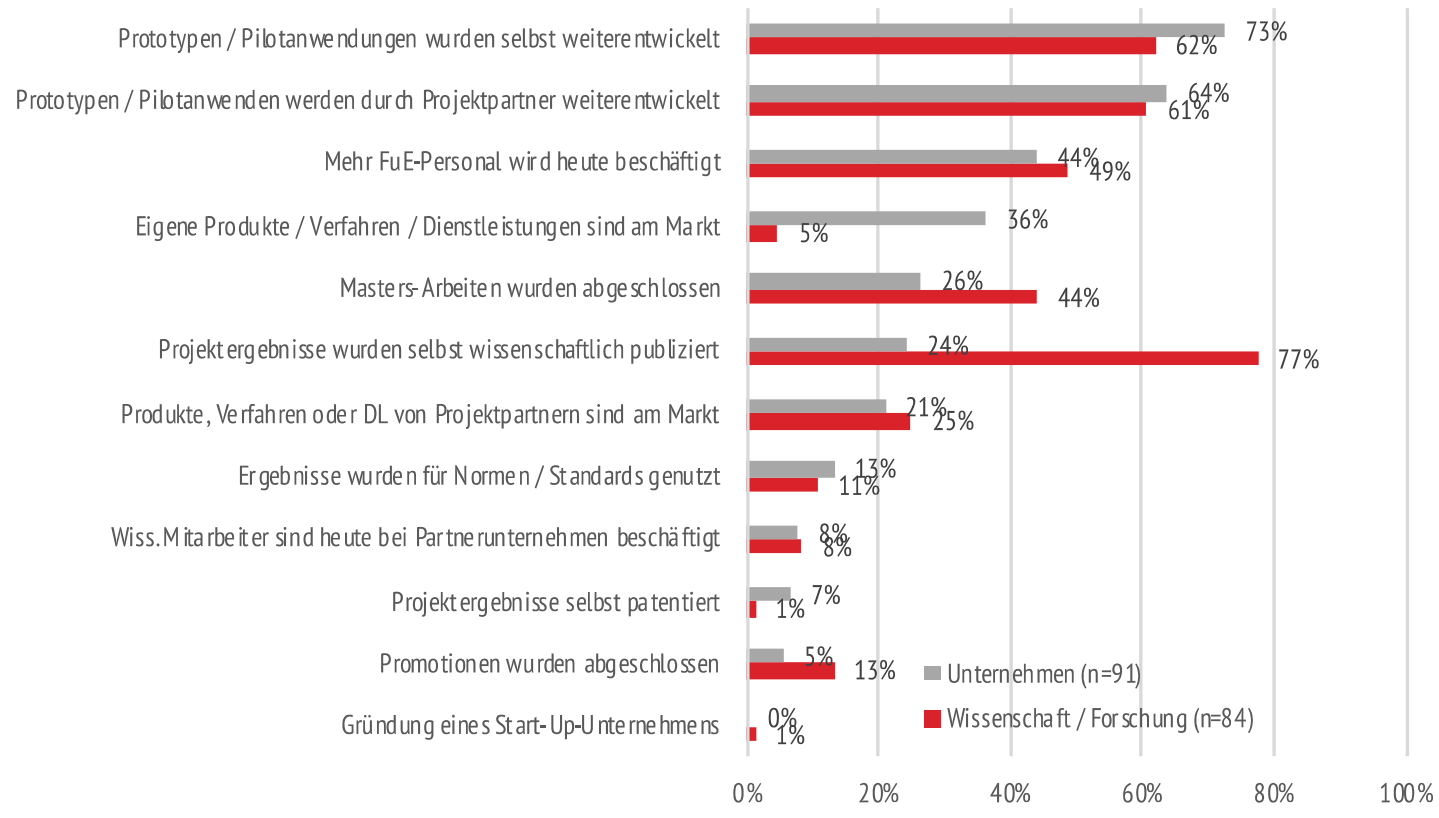

Quelle: Befragung Evaluierung BMVIT-IKT-Programme 2017; inspire research

Die Befragungsergebnisse untergliedert nach Förderschwerpunkten zeigen, dass es vor allem in den Projekten der Gemeinsamen Technologieinitiativen im vergleichsweise hohen Ausmaß gelingt, die Projektergebnisse in eigenen Produkten, Verfahren oder Dienstleistungen unmittelbar zu verwerten. Auch der von den Projektpartnern in der Industrie wahrgenommene Beschäftigungseffekt für FuE-Personal wird bei diesen Projekten von den Antwortenden am stärksten wahrgenommen. Schließlich kommt es nach den Ergebnissen der Befragung in Projekten der Gemeinsamen Technologieinitiativen auch am häufigsten zum Transfer von Wissen über Personen, die von der Wissenschaft in die Wissenschaft wechseln.

Die geringsten Anteile an Antwortenden, die bereits unmittelbare Auswirkungen der Durchführung der Projekte wahrnehmen, zeigen sich bei den Vorhaben zum Demographischen Wandel. Der Umfang der (bereits erfolgreichen) wirtschaftlichen, aber auch wissenschaftlichen Verwertung der Projekte liegt bei diesen Projekten deutlich niedriger als bei Projekten in den Förderschwerpunkten IKT der Zukunft und der Gemeinsamen Technologieinitiative.

Zusätzlich zu der Frage nach den unmittelbaren wirtschaftlichen und wissenschaftlichen Auswirkungen der Projekte befragten wir die Ansprechpartner/innen der geförderten Projekte der Themeninitiative IKT der Zukunft auch nach dem sonstigen Nutzen, die die Einrichtungen aus der Beteiligung an den Projekten bereits gezogen haben.

Die geförderten Unternehmen sehen den Nutzen der Projekte am häufigsten bei der Stärkung des Innovationsnetzwerks und der Stärkung der Partnerschaften in Österreich. Insgesamt $74 \%$ der antwortenden Vertreter/innen der Unternehmen geben diesbezüglich einen sehr großen oder großen Nutzen des Projekts an. 
Tabelle 28 Unmittelbare wirtschaftliche oder wissenschaftliche Auswirkungen der Durchführung des Projekts nach Förderschwerpunkten: Anteil der Antwortenden, die angaben, folgende Auswirkungen seien bereits sichtbar

\begin{tabular}{|c|c|c|c|c|c|c|}
\hline & \multicolumn{2}{|c|}{ IKT der Zukunft } & \multicolumn{2}{|c|}{ GTI } & \multicolumn{2}{|c|}{ Dem. Wandel } \\
\hline & $\begin{array}{l}\text { Wissen- } \\
\text { schaft } \\
(\mathrm{N}=43)\end{array}$ & $\begin{array}{c}\text { Unter- } \\
\text { nehmen } \\
(N=50)\end{array}$ & $\begin{array}{l}\text { Wissen- } \\
\text { schaft } \\
(\mathrm{N}=24)\end{array}$ & $\begin{array}{c}\text { Unter- } \\
\text { nehmen } \\
(\mathrm{N}=19)\end{array}$ & $\begin{array}{l}\text { Wissen- } \\
\text { schaft } \\
(\mathrm{N}=17)\end{array}$ & $\begin{array}{c}\text { Unter- } \\
\text { nehmen } \\
(\mathrm{N}=23)\end{array}$ \\
\hline $\begin{array}{l}\text { Prototypen / Pilotanwendungen werden } \\
\text { selbst weiterentwickelt }\end{array}$ & $63 \%$ & $68 \%$ & $67 \%$ & $84 \%$ & $53 \%$ & $70 \%$ \\
\hline $\begin{array}{l}\text { Prototypen / Pilotanwendungen werden } \\
\text { von Projektpartnern weiterentwickelt }\end{array}$ & $63 \%$ & $58 \%$ & $58 \%$ & $58 \%$ & $59 \%$ & $78 \%$ \\
\hline Mehr FuE-Personal wird heute beschäftigt & $49 \%$ & $36 \%$ & $50 \%$ & $84 \%$ & $47 \%$ & $26 \%$ \\
\hline $\begin{array}{l}\text { Eigene Produkte, Verf. o. Dienstleistungen } \\
\text { sind bereits am Markt }\end{array}$ & $7 \%$ & $28 \%$ & $4 \%$ & $63 \%$ & ०\% & $30 \%$ \\
\hline Masters-Arbeiten wurden abgeschlossen & $44 \%$ & $26 \%$ & $50 \%$ & $42 \%$ & $35 \%$ & $13 \%$ \\
\hline $\begin{array}{l}\text { Projektergebnisse wurden selbst wissen- } \\
\text { schaftlich publiziert }\end{array}$ & $84 \%$ & $20 \%$ & $83 \%$ & $37 \%$ & $53 \%$ & $22 \%$ \\
\hline $\begin{array}{l}\text { Produkte, Verf. o. Dienstleist. von Projekt- } \\
\text { partnern sind bereits am Markt }\end{array}$ & $30 \%$ & $14 \%$ & $33 \%$ & $32 \%$ & $0 \%$ & $26 \%$ \\
\hline $\begin{array}{l}\text { Ergebnisse wurden für Normen / Stan- } \\
\text { dards genutzt }\end{array}$ & $9 \%$ & $12 \%$ & $21 \%$ & $21 \%$ & $0 \%$ & $9 \%$ \\
\hline $\begin{array}{l}\text { Wissenschaftliche Mitarbeiter arbeiten } \\
\text { heute bei Partnerunternehmen }\end{array}$ & $2 \%$ & $6 \%$ & $25 \%$ & $16 \%$ & $0 \%$ & $4 \%$ \\
\hline Promotionen wurden abgeschlossen & $12 \%$ & $4 \%$ & $25 \%$ & $16 \%$ & $0 \%$ & $0 \%$ \\
\hline $\begin{array}{l}\text { Projektergebnisse wurden selbst paten- } \\
\text { tiert }\end{array}$ & $0 \%$ & $0 \%$ & $4 \%$ & $32 \%$ & $0 \%$ & $0 \%$ \\
\hline Gründung eines Start-Up-Unternehmens & $0 \%$ & $0 \%$ & $4 \%$ & $0 \%$ & $0 \%$ & $0 \%$ \\
\hline
\end{tabular}

Quelle: Befragung Evaluierung der BMVIT IKT-Programme 2017; Auswertung inspire research

Auch der Zugang zu Know-how und FuE-Ergebnissen sowie das nunmehr bessere Verständnis für die Anforderungen und Bedürfnisse der Kunden, Nutzer und Endanwender wird sehr häufig als Nutzen der Beteiligung an den Projekten genannt. Einen sehr großen oder großen Nutzen des Projekts zur Verbesserung des Marktzugangs nimmt hingegen nur ein Viertel der Unternehmen (25\%) unmittelbar wahr.

Abbildung 29 Nutzen der Beteiligung am Projekt: Anteil der Antwortenden, die den Nutzen der Projektbeteiligung als "sehr groß" oder "groß" bewerten, nach Teilnehmergruppen

Stärkung des Innovationsnet zwe rks / der Partnerschaf ten in Österreich

Zugang zu Know-how, FuE-Ergebnissen und verwandter Expertise Besse res Verständnis für die Anforderungen und Bedürfnisse der Kunden / Nutzer / Enda nwender

Möglichkeiten zur Publika tion der wissenschaftlichen Ergebnisse

Verbesse rung des Marktzugangs in Öst erre ich

Stärkung des Innovationsnet zwe rks / der Partnerschaf ten im Ausland

Verbesse rung der Ausbildung von Na chwuchswissenschaftle rn

Verbesse rung des Marktzugangs im Ausland

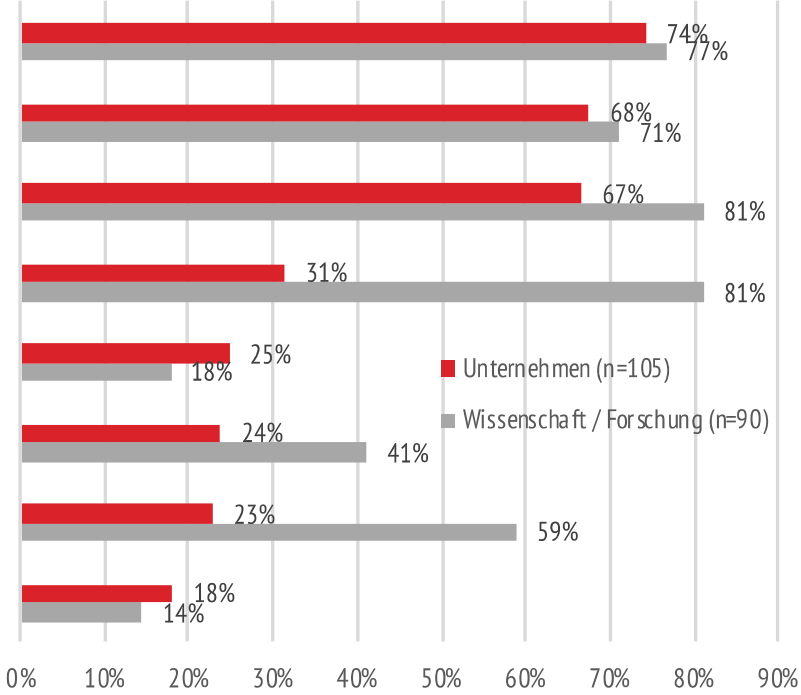

Quelle: Befragung Evaluierung BMVIT-IKT-Programme 2017; inspire research 
Eine Auswertung nach Förderschwerpunkten zeigt, dass die Projektpartner in nationalen FuE-Projekten von IKT der Zukunft den größten Nutzen ihrer Beteiligung an den Projekten in der Stärkung ihrer Innovationsnetzwerke und Partnerschaften in Österreich sehen.

Vor allem die beteiligten Unternehmen (einschließlich der sonstigen nicht gewerblichen Partner) in den Projekten zum Demographischen Wandel nehmen als Nutzen das bessere Verständnis für die Anforderungen und Bedürfnisse der Kund/innen, Nutzer/innen und Anwender/innen wahr. Auffällig ist zudem, dass die Unternehmen in Projekten zum Demographischen Wandel den Nutzen der Projekte zur Verbesserung der Ausbildung von Nachwuchswissenschaftler/innen deutlich weniger groß einschätzen als die Unternehmen mit Projekten in anderen Projekten. Gleichzeitig wird der Nutzen hinsichtlich der Publikationsmöglichkeiten von wissenschaftlichen Ergebnissen jedoch höher eingeschätzt. Auch dies ist ein Indiz dafür, dass es sich bei vielen Projekten zum Demographischen Wandel nicht um primär wissenschaftlich orientierte Forschungsprojekte handelt, sondern der Fokus der Projekte im Zusammenbringen unterschiedlicher Expertisen bzw. unterschiedlicher Akteure mit Blick auf die Erarbeitung von Lösungen für (End-)Anwenderinnen liegt, aus dem ebenfalls in Publikationen berichtet werden kann.

Die wissenschaftlichen Partner in den Projekten zum Demographischen Wandel gaben hingegen weniger häufig als wissenschaftliche Partner in Projekten anderer Förderthemen an, die Projekte hätten in "sehr großem" oder "großem" Ausmaß genutzt, Zugang zu Know-how, FuE-Ergebnissen und verwandter Expertise zu erhalten. Auch die Publikationsmöglichkeiten werden weniger positiv eingeschätzt als in anderen Förderschienen.

Tabelle 29 Nutzen der Beteiligung am Projekt nach Förderschwerpunkten: Anteil der Antwortenden, die den Nutzen der Projektbeteiligung als "sehr groß" oder "groß" bewerten, nach Förderschwerpunkten und Teilnehmergruppen

\begin{tabular}{|c|c|c|c|c|c|c|}
\hline & \multicolumn{2}{|c|}{ IKT der Zukunft } & \multicolumn{2}{|c|}{ GTI } & \multicolumn{2}{|c|}{ Dem. Wandel } \\
\hline & $\begin{array}{l}\text { Wissen- } \\
\text { schaft } \\
(\mathrm{N}=47)\end{array}$ & $\begin{array}{l}\text { Unter- } \\
\text { nehmen } \\
(\mathrm{N}=57)\end{array}$ & $\begin{array}{l}\text { Wissen- } \\
\text { schaft } \\
(\mathrm{N}=\mathbf{2 4})\end{array}$ & $\begin{array}{l}\text { Unter- } \\
\text { nehmen } \\
(\mathrm{N}=\mathbf{2 1})\end{array}$ & $\begin{array}{l}\text { Wissen- } \\
\text { schaft } \\
(\mathrm{N}=20)\end{array}$ & $\begin{array}{l}\text { Unter- } \\
\text { nehmen } \\
(\mathbf{N}=\mathbf{2 8})\end{array}$ \\
\hline $\begin{array}{l}\text { Stärkung des Innovationsnetzwerks / der } \\
\text { Partnerschaften in Österreich }\end{array}$ & $79 \%$ & $81 \%$ & $75 \%$ & $67 \%$ & $70 \%$ & $64 \%$ \\
\hline $\begin{array}{l}\text { Zugang zu Know-how, FuE-Ergebnissen } \\
\text { und verwandter Expertise }\end{array}$ & $72 \%$ & $72 \%$ & $75 \%$ & $62 \%$ & $60 \%$ & $61 \%$ \\
\hline $\begin{array}{l}\text { Besseres Verständnis für die Anforderun- } \\
\text { gen und Bedürfnisse der Kunden / Nutzer } \\
\text { / Endanwender }\end{array}$ & $89 \%$ & $65 \%$ & $79 \%$ & $48 \%$ & $60 \%$ & $82 \%$ \\
\hline $\begin{array}{l}\text { Verbesserung der Ausbildung von Nach- } \\
\text { wuchswissenschaftlern }\end{array}$ & $64 \%$ & $28 \%$ & $71 \%$ & $24 \%$ & $30 \%$ & $11 \%$ \\
\hline $\begin{array}{l}\text { Verbesserung des Marktzugangs in Öster- } \\
\text { reich }\end{array}$ & $15 \%$ & $25 \%$ & $25 \%$ & $24 \%$ & $15 \%$ & $25 \%$ \\
\hline $\begin{array}{l}\text { Möglichkeiten zur Publikation der wissen- } \\
\text { schaftlichen Ergebnisse }\end{array}$ & $79 \%$ & $25 \%$ & $96 \%$ & $33 \%$ & $65 \%$ & $43 \%$ \\
\hline $\begin{array}{l}\text { Verbesserung des Marktzugangs im Aus- } \\
\text { land }\end{array}$ & $11 \%$ & $11 \%$ & $29 \%$ & $43 \%$ & $5 \%$ & $14 \%$ \\
\hline
\end{tabular}

Diese Ergebnisse deuten schließlich auch darauf hin, dass Projekte zum Demographischen Wandel mit größeren inhaltlichen und strukturellen Herausforderungen zu kämpfen haben, als Projekte, die klassisch als Kooperationen zwischen intern forschungsaktiven Unternehmen und wissenschaftlichen Partnern organisiert sind. 


\section{Box 3: Langfristige Wirkungen der FIT-IT Förderung: $\varepsilon$ CEDAC (Evolution Control Environment for Distributed Automation Components)}

Das Projekt $\varepsilon$ CEDAC wurde in den Jahren 2005 bis 2007 durchgeführt. Es wurde in der 4. Ausschreibung der FIT-IT Programmlinie Embedded Systems durch PROFACTOR als Koordinator mit den Projektpartnern TU Wien (Institut für Automatisierungs- und Regelungstechnik), Bachmann electronic, kircher SOFT (heute logi.cals), Siemens VAI Metals Technologies (heute Primetals Technologies) und LOYTEC electronics eingereicht. Im Zentrum des Projekts stand die Entwicklung von adaptiven Steuerungssystemen im industriellen Bereich.

An der TU Wien hat das Projekt gemeinsam mit anderen FIT-IT geförderten Vorhaben wesentlich dazu beigetragen, die Forschungsgruppe personell zu verdreifachen. Das Projekt bot substanzielle Möglichkeiten für wissenschaftliche Veröffentlichungen und Konferenzbeiträge. Es arbeiteten auch mehrere Doktoranden am $\varepsilon$ CEDAC-Projekt mit, wobei einer der Kandidaten seine Promotion sub auspiciis abschließen konnte.

Eines der Arbeitsergebnisse aus dem Projekt \&CEDAC bildete gemeinsam mit den Ergebnissen aus dem bereits zuvor gestarteten FIT-IT Projekt $\mu$ Crons die Grundlage für das Entwicklungswerkzeug 4Diac (www.eclipse.org/4diac/), das eine Standard-kompatible Open Source Infrastruktur für verteilte industrielle Mess- und Steuerungssysteme bietet. 4diac wird heute weltweit von Universitäten und Forschungseinrichtungen in Forschung und Lehre für Anwendungen der verteilten Automatisierungstechnik eingesetzt. Es sind aber auch Produkte gewerblicher Anbieter am Markt erhältlich, die das Entwicklungswerkzeug 4Diac nutzen. Beispielsweise bietet das österreichische Unternehmen nxtControl eine Automatisierungssoftware für Industrie4.0-Anwendungen an, die auf der 4Diac-Plattform aufbaut. Das Grazer Unternehmen meo Energy nutzt 4Diac als Plattform in intelligenten und modularen Heizungssystemreglern.

Der Projektpartner Bachmann electronics nutzte ebenfalls 4Diac für weitere eigene Entwicklungen. Außerdem bestehen nach wie vor Arbeitskontakte mit logi.cals. Auch mit PROFACTOR wurde die Zusammenarbeit nach Projektende fortgesetzt bzw. Kooperationen bestehen bis heute.

Der Projektpartner Siemens VAI Metals Technologies konnte hingegen die Entwicklungen nicht im eigenen Unternehmen nutzen. Im Zuge der Änderung der Eigentumsverhältnisse am Unternehmen während der Laufzeit des \&CEDAC-Projekts wurde auch das im Unternehmen verwendete Automatisationssystem durch jenes der neuen Konzernmutter ersetzt. Die Ergebnisse des \&CEDAC-Projekts, die sich technisch auf das ursprünglich im Unternehmen eingesetzte Automatisierungssystem bezogen, hatten daher keine Relevanz mehr.

logi.cals führte die Entwicklungen aus dem Projekt عCEDAC nach Projektende nicht weiter. Das war für das Unternehmen beim Start des Projekts aber auch nicht das Hauptziel gewesen. Vielmehr ging es dem Unternehmen darum, nach vielen Jahren des "Schwimmens in der eigenen Suppe" erstmals wieder Forschungskooperationen mit wissenschaftlichen Einrichtungen und anderen Unternehmen einzugehen. Damit wollte das Unternehmen den Horizont für die eigenen Innovationsvorhaben verbreitern. Die Forschungskooperation zwischen kircher SOFT (logi.cals) und der TU Wien wurden nach Projektende auch tatsächlich weiter aufrecht erhalten und mündeten unter anderem im Jahr 2010 in die Beteiligung am Christian Doppler-Labor "Software Engineering Integration für flexible Automatisierungssysteme" an der TU Wien. 


\section{Ergebnisse und Wirkungen von Leitprojekten}

Auch wenn die beiden bisher geförderten Leitprojekte der Themeninitiative "IKT der Zukunft" noch nicht abgeschlossen sind, geben die Gespräche mit Projektbeteiligten des Leitprojekts 2014 und die entsprechenden Rückmeldungen aus der Befragung bereits erste Hinweise auf die erzielten Effekte und den Mehrwert dieses neuen Förderinstruments im Vergleich zu klassischen kooperativen FuE-Projekten.

Bezüglich der Bewertung der wirtschaftlichen Effekte ist es allerdings noch zu früh, ein abschließendes Urteil zu fällen. Einige Projektteilnehmer berichteten jedoch in der Befragung darüber, dass ihre Einrichtung aufgrund der Teilnahme am Leitprojekt, heute mehr FuE-Personal beschäftige. Zwei von fünf Partnern, für die uns aus der Befragung Antworten zum Leitprojekt 2014 vorliegen, gaben an, bereits Produkte oder Dienstleistungen am Markt zu haben, die auf Ergebnissen des Projekts aufbauen. Zwei weitere Partner entwickeln ihre Prototypen und Pilotanwendungen aus dem Projekt aktuell noch weiter. Ansonsten werden von den Unternehmen und sonstigen (nichtwissenschaftlichen) Partnern des Leitprojekts 2014 bisher noch im geringen Umfang weitere unmittelbare wirtschaftliche oder wissenschaftliche Auswirkungen des Projekts in der eigenen Einrichtung wahrgenommen. Bei den antwortenden Einrichtungen handelte es sich um potenzielle Anbieter der zu entwickelten Prototypen und Pilotanwendungen.

Allerdings haben Leitprojekte auch eine Funktion, die deutlich über die Generierung von Nutzen beim einzelnen geförderten Partner hinausgeht. Leitprojekte sollen Infrastruktur aufbauen und einen Referenzrahmen bieten, der es auch dritten Einrichtungen erlaubt anzudocken und eigene Innovationen voranzutreiben. Im ersten Leitprojekt Data Market Austria wurde dazu beispielsweise ein begleitendes Inkubator-Programm aufgesetzt, das es KMU und Start-ups erlauben soll, prototypische Anwendungen für datengetriebene Geschäftsmodelle zu testen und weiterzuentwickeln. Auch die angebotenen Workshops, Webinare und sonstige Veranstaltungen gehen sehr deutlich über das Niveau hinaus, das bei kooperativen FuE-Projekten erwartet werden kann.

Ein Unternehmenspartner des Leitprojekts Data Market Austria, der vor allem die Softwareentwicklung von Lösungen unterstützt, wies im Gespräch auf eine große Resonanz der Transferkomponente des Leitprojekts über die Veranstaltungen und Begleitmaßnahmen hin. Die bisher durchgeführten Informationsveranstaltungen und Präsentationen zu den Pilotanwendungen seien auf starkes Interesse dritter Unternehmen gestoßen. Man habe bereits zahlreiche Anfragen von neuen Unternehmen nach weiteren Pilotanwendungen bzw. Diensten erhalten. Mit klassischen FuE-Projekten hätte man wahrscheinlich keine vergleichbaren Ergebnisse erzielen können. Das Konzept des Instruments Leitprojekt dürfte damit aufgegangen bzw. ein wesentliches Ziel der Förderung bereits erreicht worden sein.

\subsection{Wissenschaftliche Publikationen}

In welchem Umfang publizierten die an FIT-IT Projekten beteiligten Wissenschaftler/innen? In welchem Umfang publizieren die Wissenschaftler/innen an den Forschungseinrichtungen gemeinsam mit Unternehmen? Änderte sich das Publikationsverhalten während oder nach der Durchführung der FIT-IT Projekte? Unterscheiden sich geförderte und nicht-geförderte Wissenschaftler/innen hinsichtlich des Umfangs ihrer Publikationstätigkeit nach dem (geplanten) Projektstart?

Für unsere Auswertungen zu diesen Fragen betrachten wir die in Scopus erfassten Publikationen der technischen Ansprechpersonen der wissenschaftlichen Einrichtungen in FIT-IT 
Anträgen in drei Dreijahresperioden: 1) Publikationen, die im Jahr des (geplanten) Starts des FIT-IT Projekts und in den zwei Kalenderjahren zuvor veröffentlicht wurden (Phase vor der Projektdurchführung); 2) Publikationen, die im ersten bis dritten Kalenderjahr nach dem Jahr des (geplanten) Starts des FIT-IT Projekts veröffentlicht wurden (Projektphase) und 3) Publikationen, die im vierten bis sechsten Kalenderjahr nach dem Jahr des (geplanten) Starts des FIT-IT Projekts veröffentlicht wurden (Phase nach der Projekt).

\section{Anteil der Publikationen gemeinsam mit Unternehmen}

Betrachten wir das Ausmaß der Publikationstätigkeit zwischen den technischen Ansprechpersonen und Koautoren aus der Wirtschaft über den gesamten Beobachtungszeitraum hinweg, zeigen sich zwischen der Gruppe der technischen Ansprechpersonen mit genehmigten Projekten und jener mit nur abgelehnten Anträgen kaum Unterschiede. Etwas mehr als jede sechste Publikation der technischen Ansprechpersonen von Anträgen in den FIT-IT-Programmlinien (18 \% bzw. 17 \%) wurde gemeinsam mit einem Autor / einer Autorin aus der Wirtschaft / Praxis veröffentlicht. Die technischen Ansprechpersonen in Anträgen der Gemeinsamen Technologieinitiative veröffentlichten mehr als jede vierte Publikation (27\% bzw. 29 \%) mit einem Partner / einer Partnerin aus der Wirtschaft / Praxis. Den geringsten Anteil an Publikationen mit der Wirtschaft / Praxis sehen wir bei den technischen Ansprechpersonen der Projekte zum Demographischen Wandel (10 \% bzw. $13 \%$ ).

Abbildung 30 Anteil der Publikationen mit einem Koautor aus der Wirtschaft / Praxis der technischen Ansprechpersonen der wissenschaftlichen Partner in FIT-IT Anträgen nach Programmen

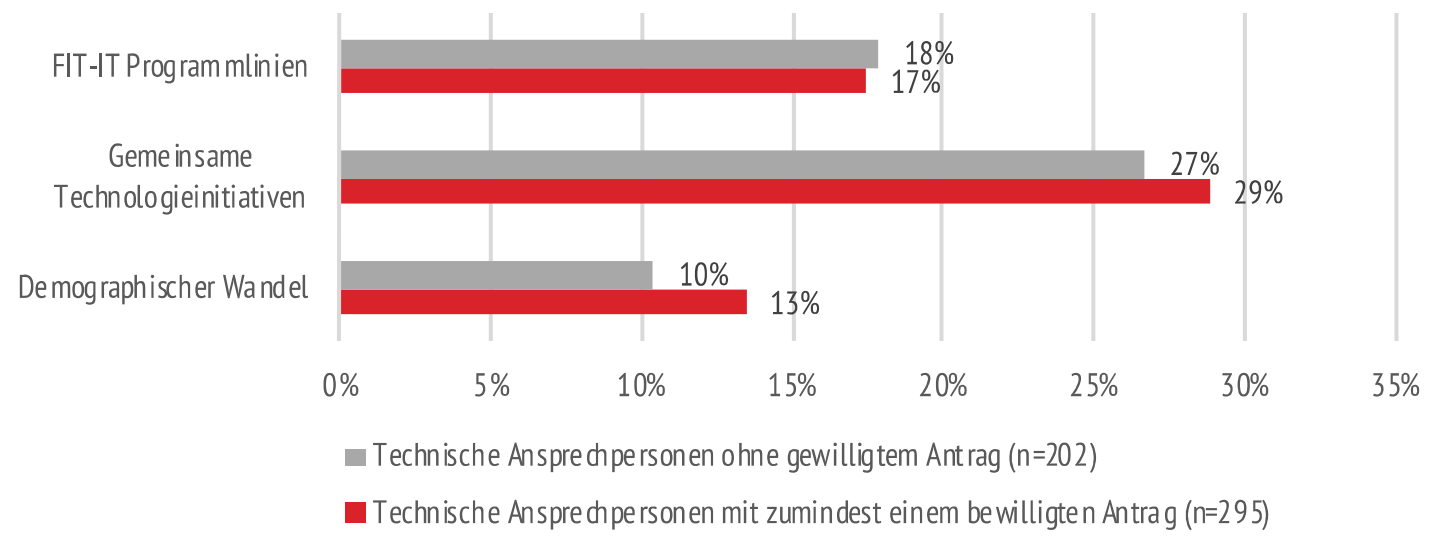

Quelle: FFG, Scopus; Auswertung und Darstellung inspire research

\section{Publikationen gemeinsam mit Unternehmenspartnern von FIT-IT Projekten}

In welchem Umfang publizierten die technischen Ansprechpersonen genehmigter und nichtgenehmigter Anträge mit jenen Partnern aus Wirtschaft und Praxis, mit denen sie die FIT-IT Anträge gemeinsam eingereicht haben?

Gemessen an allen Publikationen mit Partnern aus der Wirtschaft und Praxis macht der Anteil der Publikationen, die gemeinsam mit Unternehmen, mit denen in den FIT-IT-Projekten zusammengearbeitet wurde (bzw. geplant war zusammenzuarbeiten), über den gesamten betrachteten Publikationszeitraum hinweg rund ein Viertel. Während bei den technischen Ansprechpersonen, die keine FIT-IT Förderung erhalten haben, dieser Anteil über die drei betrachteten Publikationsphasen hinweg stetig zunimmt - ein Indiz dafür, dass es einem Teil der nicht geförderten wissenschaftlichen Partner gelingt, geplante Kooperationen auch aus anderen Quellen zu finanzieren - sieht man in der Gruppe der geförderten technischen Ansprechpersonen einen deutlichen Sprung des Anteils der gemeinsamen Publikationstätigkeit 
in der Projektphase. Diesen Sprung interpretieren wir als unmittelbare Wirkung der FIT-ITFörderung.

Abbildung 31 Anteil der Publikationen der technischen Ansprechpersonen der wissenschaftlichen Partner gemeinsam mit Koautoren aus jenen Unternehmen, mit denen in FIT-IT-Projekten zusammengearbeitet wurden, an allen Publikationen mit Partnern aus der Wirtschaft, nach Publikationsperiode

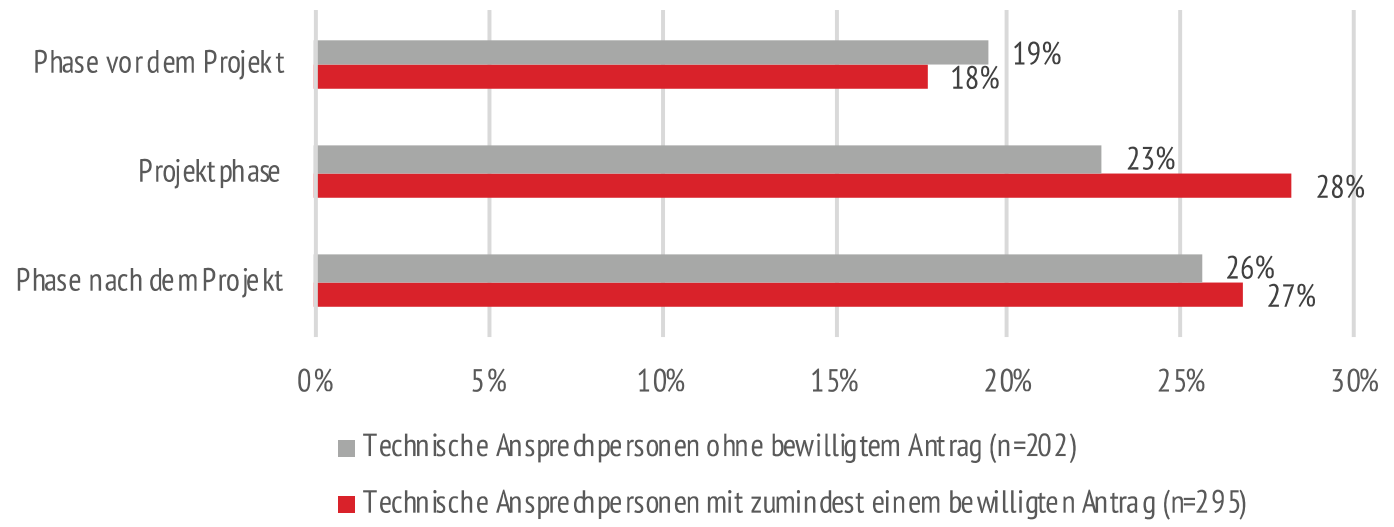

Quelle: FFG, Scopus; Auswertung und Darstellung inspire research

Deutlich größer fällt dieser Unterschied im Anteil gemeinsamer Publikationen mit Unternehmen in den FIT-IT-Projekten zwischen geförderten und nicht-geförderten technischen Ansprechpersonen aus, wenn wir nicht die Anzahl der Publikationen betrachten, sondern die technischen Ansprechpersonen selbst.

Während in der Phase vor dem Projekt 11 \% der technischen Ansprechpersonen der wissenschaftlichen Partner zumindest eine gemeinsame Publikation mit FIT-IT Unternehmenspartnern aufweisen, steigt dieser Anteil bei geförderten technischen Ansprechpersonen auf $25 \%$ in der Förderphase an. Der entsprechende Anteil unter den nicht-geförderten technischen Ansprechpersonen liegt hingegen bei nur $13 \%$. Auch in der Phase nach der Projektdurchführung finden wir noch einen Anteil von 21 \% der geförderten technischen Ansprechpersonen, die gemeinsam mit einem am Projekt beteiligten Unternehmen publiziert haben. In der Gruppe der nicht-geförderten technischen Ansprechpersonen liegt dieser Anteil bei $12 \%$.

Abbildung 32 Anteil der technischen Ansprechpersonen der wissenschaftlichen Partner, die gemeinsam mit den an gemeinsamen FIT-IT Projekten beteiligten Unternehmen publiziert haben, nach Publikationsphase

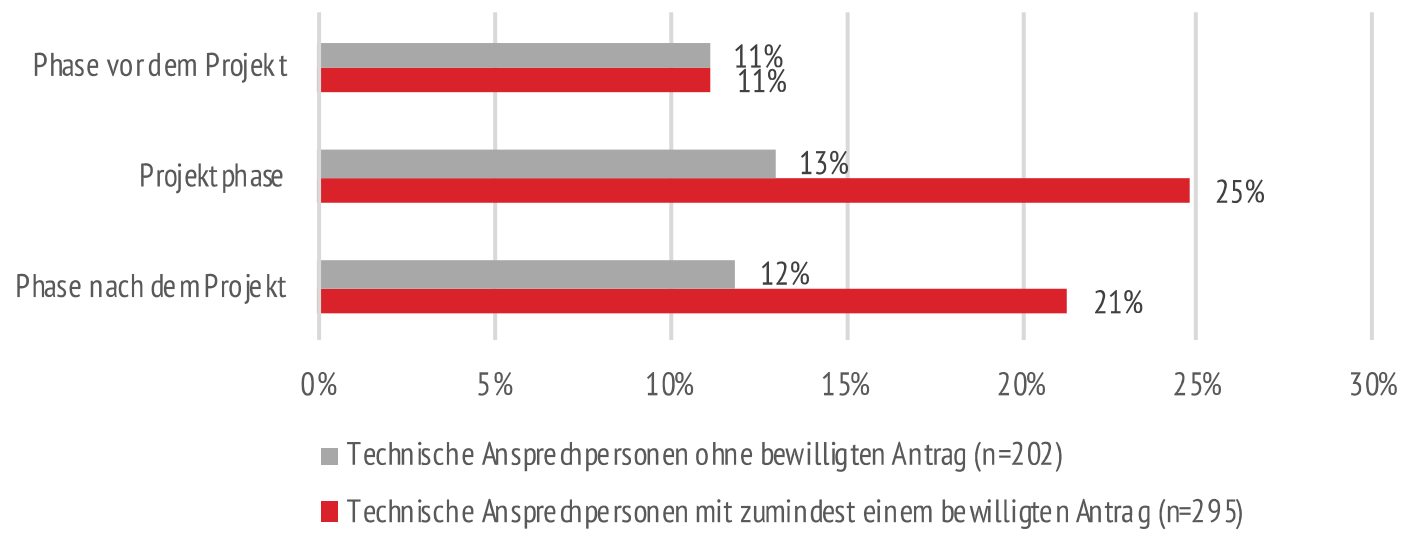

Quelle: FFG, Scopus; Auswertung und Darstellung inspire research

Die Auswertung der Publikationsdaten deutet damit auf eine deutliche Zunahme der gemeinsamen Publikationstätigkeit zwischen den wissenschaftlichen Einrichtungen und den an den FIT-IT Projekten beteiligten Unternehmen hin. 


\section{Box 4: Langfristige Wirkungen der FIT-IT Förderung: IDIOM (Informationsdiffusion zwischen interaktiven Online-Medien)}

Das Projekt IDIOM wurde in den Jahren 2006 bis 2009 unter Koordination des Instituts für Wissensmanagement der Technischen Universität Graz durchgeführt. Als weitere Partner waren die Wirtschaftsuniversität Wien, die Software Unternehmen Gentics Software und Prisma Solutions sowie Austria.info Systems, eine Tochtergesellschaft von Österreich Werbung, am Projekt beteiligt. Das Vorhaben wurde in der 3. Ausschreibung der FIT-IT Programmlinie Semantic Systems eingereicht.

Das Forschungsteam hat über das Projekt und die Projektergebnisse in großem Umfang wissenschaftlich publiziert. Alleine für den Koordinator des Projekts finden sich für den Projektzeitraum in Scopus 20 Publikationen, die mit dem Thema Semantic Web zu tun haben. Allerdings konnte das Kernprojektteam bereits auf Ergebnisse aufbauen, die im Rahmen des ebenfalls aus FIT-IT Mitteln geförderten Projekts AVALON erarbeitet wurden.

Das IDIOM Projekt trug dazu bei - in Kombination mit weiteren Forschungsprojekten im selben Themengebiet - zwei Nachwuchswissenschaftlern die Dissertation zu ermöglichen. Beide sind nach wie vor an Universitäten als Post-doc bzw. Professor tätig und forschen weiterhin an der Entwicklung semantischer Technologien. Auch die leitenden projektdurchführenden Wissenschaftler des IDIOM-Projekts haben als Professoren an Hochschulen die Arbeiten im Rahmen mehrerer nationaler und EU-Forschungsprojekte fortgesetzt. Einer der am Projekt beteiligten Wissenschaftler nutzt heute als Professor an einer Schweizer Fachhochschule das in IDIOM aufgebaute Know-how regelmäßig in Innovationsprojekten gemeinsam mit Schweizer Unternehmen.

Ein unmittelbares Ergebnis des Projekts war die Gründung des Spin off-Unternehmens WebLyzard, das Lösungen für die Marktforschung, strategische Positionierung und die Messung des Kommunikationserfolgs auf Grundlage der visuell unterstützten Analyse von (Web-)Daten anbietet. Die Applikation Webmonitor erlaubt beispielsweise die Echtzeitanalyse von Online-Nachrichten, um Meinungsführer/innen zu identifizieren, die Auswirkungen von Medienkampagnen und Ereignissen zu bewerten und die Wahrnehmung bestimmter Themen in Abhängigkeit von diesen Ereignissen unter verschiedenen Stakeholdergruppen zu vergleichen. Zu den Kunden von WebLyzard zählt beispielsweise die National Oceanic and Atmospheric Administration (NOAA), die für ihre Web Intelligence Lösungen unter anderem auf das Know-how des Unternehmens zurückgreift.

Die Rolle von Austria.info Systems im Projekt war in erster Linie, als Multiplikator für die österreichische Tourismusbranche zu fungieren. Da Tourismus- und Tourismuswerbeunternehmen im Vergleich zu anderen Branchen nur wenige Erfahrung mit Forschung und Forschungszusammenarbeit hatten, war es Österreich Werbung über die Tochter Austria.info Systems wichtig, ein Zeichen für die hohe Bedeutung des Themas zu setzen. Für Austria.info Systems brachte das Projekt aber keine unmittelbar im Unternehmen verwertbaren Ergebnisse, sondern trug dazu bei, die Aufmerksamkeit und Bedeutung von Semantic Web Lösungen für den Tourismus insgesamt zu erhöhen.

Das Thema Semantic Web ist im Tourismus auch heute noch hochaktuell. Applikationen wie Sprachassistenzsysteme und Modelle zur intelligenten Informationsaufbereitung touristischer Informationen spielen eine immer größere Rolle in der Kommunikation mit potenziellen Kund/innen. Neue Forschungsimpulse kommen hier insbesondere auch aufgrund der hohen strategischen Bedeutung von Big Data und Artificial Intelligence für Wissenschaft und Wirtschaft. 


\section{Publikationstätigkeit der Unternehmen und Anwendungspartner}

Betrachten wir die Partner aus Wirtschaft und Praxis (d. h. Unternehmen und sonstige Partner, die keine Hochschulen oder Forschungseinrichtungen sind), die an den Anträgen während der Laufzeit des Impulsprogramms FIT-IT beteiligt waren, so zeigt sich, dass mehr als ein Viertel (28\%) aller geförderter Partner gemeinsame, in Scopus erfasste Publikationen mit technischen Ansprechpersonen wissenschaftlicher Einrichtungen, die an FIT-IT beteiligt waren, vorweisen kann. Interessanterweise sind das nicht nur Publikationen mit den technischen Ansprechpersonen in gemeinsam eingereichten Projekten, sondern auch Publikationen mit technischen Ansprechpersonen, die an anderen FIT-IT-Projekten beteiligt waren. Als Bezugszeitraum zur Bestimmung relevanter Publikationen haben wir wieder den Zeitraum von zwei Kalenderjahren vor dem Kalenderjahr des (geplanten) Starts des FIT-IT Projekts bis sechs Kalenderjahre nach dem Kalenderjahr des (geplanten) Starts des FIT-IT Projekts herangezogen.

Abbildung 33 Anteil der Partner (Unternehmen, sonstige Einrichtungen), die mit den an FIT-IT Projekten beteiligten wissenschaftlichen Einrichtungen gemeinsam publiziert haben*

Anteil Unternehmen mit Ko-Publika tionen gemeinsam mit Anspr echpartner bei wissenschaft lichen Partnern in a llen FITIT Anträgen

Anteil Unternehmen mit Ko-Publikationen gemeinsam mit Ansprechpartner bei wissenschaft lichen Partnern in eigenen FIT-IT Anträgen

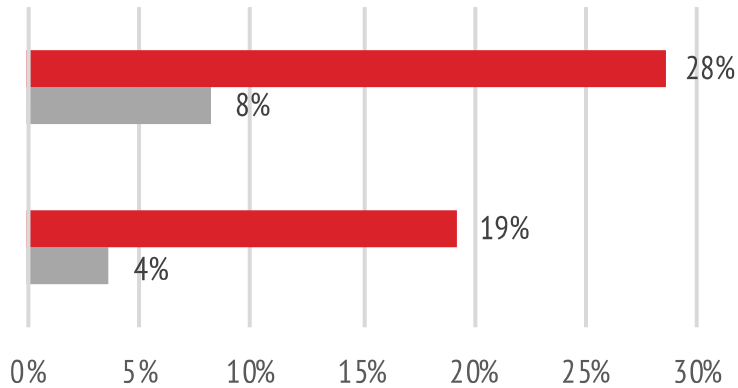

- Partner mit ge nehmigten FIT-IT-Anträ gen ( $n=148)$ — Partner ohne genehmigte FIT-IT Anträge $(n=42)$

Basis: alle in Scopus erfassten Publikationen der technischen Ansprechpartner wissenschaftlicher Einrichtungen von FITIT-Projektanträgen im Zeitraum von zwei Kalenderjahren vor dem Kalenderjahr des (geplanten) Beginns des Projekts bis einschließlich sechs Kalenderjahre nach dem Kalenderjahr des (geplanten) Beginns des FIT-IT-Projekts Quelle: FFG, Scopus; Auswertung und Darstellung inspire research

Insgesamt findet sich eine hohe Anzahl von Publikationen der technischen Ansprechpersonen bei den wissenschaftlichen Partnern (Hochschulen, außeruniversitäre Forschungseinrichtungen), die gemeinsam mit Unternehmenspartnern oder anderen, an den Projektanträgen beteiligten Einrichtungen veröffentlicht wurden. Überrascht hat uns in diesem Zusammenhang ein Ergebnis besonders: Insgesamt 56 \% der identifizierten Publikationen, die gemeinsam zwischen einem Unternehmen und einem Autor /einer Autorin, der/die als technische Ansprechperson bei einem wissenschaftlichen Partner in einem FIT-IT Antrag aufscheint, zeigen eine Zusammenarbeit, für die in dieser Kombination kein FIT-IT-Projekt beantragt wurde.

Zu einem gewissen Ausmaß kann es natürlich auch sein, dass die Unternehmen mit Arbeitsgruppen an Universitäten und Forschungseinrichtungen zusammenarbeiteten, für die bei verschiedenen Projekten unterschiedliche technische Ansprechpersonen angeführt sind. Die bibliographische Auswertung legt aber auch den Schluss nahe, dass eine Zusammenarbeit zwischen den Unternehmen und den Arbeitsgruppen der Hochschulen und außeruniversitären Forschungseinrichtungen in substanziellen Umfang auch außerhalb der (geförderten) FIT-IT-Anträge stattgefunden hat, entweder im Rahmen anderer Förderprogramme oder auch nicht gefördert. Die Tabelle auf der nächsten Seite gibt einen Überblick über die publikationsintensivsten Unternehmen aus Kooperationen mit technischen Ansprechpersonen von wissenschaftlichen Partnern im Impulsprogramm FIT-IT. 
Tabelle 30 Rangliste der an FIT-IT beteiligten Unternehmen nach Anzahl der Ko-Publikationen mit technischen Ansprechpersonen (TA) von wissenschaftlichen Einrichtungen in FIT-IT Anträgen*

\begin{tabular}{|c|c|c|c|c|}
\hline Unternehmen & $\begin{array}{l}\text { Publikationen } \\
\text { mit TA von wiss. } \\
\text { Partnern }\end{array}$ & $\begin{array}{l}\text { davon mit TA in } \\
\text { eigenen FIT-IT } \\
\text { Anträgen }\end{array}$ & $\begin{array}{l}\text { Anzahl der TA, } \\
\text { mit denen pu- } \\
\text { bliziert wurde }\end{array}$ & $\begin{array}{c}\text { Anzahl der be- } \\
\text { willigten FIT-IT } \\
\text { Anträge }\end{array}$ \\
\hline Infineon Technologies Austria & 597 & 298 & 61 & 40 \\
\hline Siemens Austria & 444 & 141 & 91 & 15 \\
\hline AVL List & 161 & 67 & 41 & 14 \\
\hline DICE & 137 & 102 & 7 & 7 \\
\hline NXP Semiconductors & 136 & 72 & 27 & 10 \\
\hline Voestalpine & 132 & 42 & 21 & 0 \\
\hline Microsoft & 100 & 7 & 32 & 1 \\
\hline Boehler & 96 & 19 & 10 & 1 \\
\hline ams AG & 86 & 18 & 18 & 12 \\
\hline BIOS Bioenergiesysteme & 86 & 86 & 2 & 1 \\
\hline CISC Semiconductors & 85 & 52 & 12 & 4 \\
\hline Philips & 68 & 2 & 24 & 1 \\
\hline Medexter Healthcare & 45 & 39 & 3 & 1 \\
\hline Oregano Systems & 44 & 36 & 6 & 3 \\
\hline TTTech & 41 & 33 & 15 & 12 \\
\hline Intel Austria & 40 & 0 & 18 & 2 \\
\hline Tritonic & 37 & 0 & 3 & 1 \\
\hline A1 Telekom Austria & 33 & 11 & 11 & 6 \\
\hline Frequentis & 32 & 3 & 14 & 0 \\
\hline Lixto & 32 & 32 & 4 & 5 \\
\hline Robert Bosch & 31 & 5 & 14 & 0 \\
\hline $\begin{array}{l}\text { Team Communication Technol- } \\
\text { ogy Management }\end{array}$ & 31 & 29 & 4 & 2 \\
\hline Thales & 27 & 0 & 17 & 3 \\
\hline Rockwell & 27 & 8 & 8 & 1 \\
\hline Kapsch & 25 & 2 & 10 & 3 \\
\hline SLR Engineering & 22 & 18 & 5 & 1 \\
\hline Ximes & 22 & 5 & 3 & 1 \\
\hline Weblyzard & 20 & 20 & 5 & 2 \\
\hline Abatec & 19 & 19 & 1 & 0 \\
\hline DMCE & 18 & 0 & 6 & 5 \\
\hline Alcatel Lucent & 17 & 0 & 13 & 1 \\
\hline Seekda & 17 & 14 & 5 & 2 \\
\hline Matrixware & 17 & 8 & 4 & 0 \\
\hline Fronius & 16 & 4 & 9 & 1 \\
\hline Airbus & 15 & 0 & 7 & 1 \\
\hline Plansee & 15 & 0 & 6 & 1 \\
\hline Magna & 14 & 1 & 12 & 3 \\
\hline Netural & 14 & 4 & 6 & 2 \\
\hline Andritz & 14 & 1 & 4 & 1 \\
\hline ITH icoserve & 13 & 13 & 1 & 1 \\
\hline Imagination Computer Services & 12 & 7 & 7 & 3 \\
\hline Ferrobotics & 12 & 12 & 3 & 3 \\
\hline
\end{tabular}

* Publikationszeitraum: Zwei Kalenderjahre vor dem Jahr des (geplanten) FIT-IT Projekts bis sechs Kalenderjahre nach dem Jahr des (geplanten) FIT-IT Projekts; Quelle: FFG, Scopus, Auswertung inspire research 
Die bibliometrischen Auswertungen zeigen zusammen mit den Angaben der Förderempfänger in der Befragung ein insgesamt hohes Ausmaß an wissenschaftlicher Verwertung der Projektergebnisse durch die wissenschaftlichen Einrichtungen und durch die an den Projekten beteiligten Unternehmen. Mehr als $80 \%$ der technischen Ansprechpersonen der wissenschaftlichen Partner gaben in der Befragung an, selbst Ergebnisse aus den Projekten wissenschaftliche publiziert zu haben. Geförderte technische Ansprechpersonen publizierten in den drei Kalenderjahren nach dem Jahr des Starts des FIT-IT Projekts durchschnittlich 14,2 Publikationen; die vorgesehenen technischen Ansprechpersonen in abgelehnten FIT-IT Anträgen publizierten durchschnittlich 10,1 Publikationen. In der Projekt- und Postprojektphase (erstes bis sechstes Kalenderjahr nach dem Jahr des Starts des FIT-IT Projekts) finden sich in Scopus insgesamt 530 Ko-Publikationen der technischen Ansprechpersonen wissenschaftlicher Einrichtungen mit Unternehmenspartnern in den Projekten. Selbst unter der Annahme, dass nur ein Viertel des Publikationsoutputs der technischen Ansprechpersonen der wissenschaftlichen Einrichtungen in der Projektphase unmittelbar den FIT-IT Projekten zugerechnet werden kann, würde damit der in den Programmzielen angestrebte Wert von drei Publikationen je Projekt jedenfalls deutlich übertroffen worden sein.

\subsection{Patentanmeldungen der beteiligten Einrichtungen}

In welchem Umfang patentierten die an Anträgen in den BMVIT IKT-Programmen beteiligten Einrichtungen ihre Erfindungen? In welchem Umfang waren auch wissenschaftliche Partner an den Patenten der Unternehmen beteiligt? Unterscheiden sich geförderte und nicht-geförderte Unternehmen hinsichtlich des Umfangs ihrer Patentierungstätigkeit? Welche Bedeutung haben unterschiedliche Technologiefelder in den Patentanmeldungen, die von an den BMVIT IKT-Programmen beteiligten Unternehmen angemeldet wurden? Wie hat sich der Anteil der Patente in IKT-Technologiefeldern seit Start des Impulsprogramms FIT-IT entwickelt?

Für die folgenden Patentanalysen haben wir die Datenbank PATSTAT des Europäischen Patentamts herangezogen. In einem ersten Schritt wurden aus der PATSTAT-Datenbank alle Anmelder/innen oder Erfinder/innen mit einer österreichischen Adresse identifiziert. In diesem Datenbankauszug wurde nach den an Projektanträgen in den BMVIT IKT-Programmen beteiligten Einrichtungen gesucht und die entsprechenden Datenbankeinträge seit dem Jahr 2002 gesammelt.

\section{Anmeldungen durch Unternehmen und Forschungseinrichtungen}

Insgesamt finden sich für $31 \%$ der in den BMVIT IKT-Programmen geförderten Unternehmen und für 22 \% der nicht-geförderten Unternehmen PATSTAT-Einträge zu Patentanmeldungen. Zwischen den Programmen zeigen sich sehr deutliche Unterschiede. Während fast drei Viertel (74 \%) der geförderten Unternehmen in den Gemeinsamen Technologieinitiativen im Zeitraum 2002 bis 2016 Patente angemeldet haben, liegt der Anteil unter den Unternehmen in Projekten zum Demographischen Wandel bei $15 \%$.

Die an den Projekten der nationalen FIT-IT bzw. IKT der Zukunft-Förderung beteiligten Unternehmen haben zu $37 \%$ einen PATSTAT-Eintrag. Untern den geförderten Unternehmen finden sich in allen drei Programmen bzw. Förderthemen höhere Anteile an Patentanmeldern, wobei die Unterschiede zwischen geförderten und nicht-geförderten Unternehmen in Projekten der Gemeinsamen Technologieinitiativen am größten sind und in Projekten zum Demographischen Wandel am geringsten. 


\section{Box 5: Langfristige Wirkungen der FIT-IT Förderung: GenOptikum (Visual Data Mining for Genetic Data)}

Das Projekt GenOptikum wurde in den Jahren 2007 bis 2009 unter Koordination des Instituts für Wissensmanagement der Technischen Universität Graz durchgeführt. Als weitere Partner waren das Institut für Pathologie an der Medizinischen Universität Graz sowie das Spin off-Unternehmen Oridis Biomed am Projekt beteiligt. Die Förderung erfolgte im Zuge der 1. Ausschreibung der FIT-IT Programmlinie Visual Computing. Die Visualisierung von biologischer bzw. medizinischen Informationen war zum Zeitpunkt der Einreichung des Projekts ein junges Forschungsthema. Inzwischen wurden international an vielen Universitäten neue Professuren bzw. Forschungsgruppen eingerichtet, die sich mit bioinformatischer Visualisierung beschäftigen. In diesem Sinne sei GenOptikum nach Ansicht beteiligter Personen ein wegweisendes Projekt gewesen.

Die beiden Doktoranden, die an der TU Graz im GenOptikum-Projekt tätig waren, haben inzwischen ihre akademische Karriere sehr erfolgreich fortsetzen können. Ein ehemaliger Doktorand ist inzwischen als Assistenzprofessor an der University of Utah beschäftigt und leitet das Visualization Design Lab am Scientific Computing and Imaging Institute / School of Computing. Der zweite ehemals im Projekt tätige Doktorand ist heute Professor am Institut für Computergraphik an der Johannes Kepler Universität Linz.

Ihre wissenschaftliche Zusammenarbeit haben beide auch nach Ende des Projekts GenOptikum fortgesetzt. Ein für Dritte nutzbares Ergebnis dieser Zusammenarbeit ist die Open Source Software Caleydo (www.caleydo.org), die den Nutzer/inne/n die Entwicklung von Tools für die Visualisierung von biomolekularen Informationen ermöglicht. Ergebnisse des GenOptikum Projekts sind in die Entwicklung von Caleydo mit eingeflossen. Die zugrundeliegenden Forschungsarbeiten wurden dabei nicht nur aus öffentlichen Mitteln nationaler und regionaler Fördergeber, sondern auch durch die Beiträge von Unternehmenspartnern wie Novartis oder Boehringer Ingelheim finanziert. Caleydo-Anwender finden sich sowohl im akademischen Bereich als auch in der industriellen Forschung.

Mit weiteren Partnern aus ihrem Kollaborationsumfeld haben die beiden ehemaligen Doktoranden ein Spin off-Unternehmen gegründet. Das 2016 gegründete Unternehmen datavisyn entwickelt Softwarelösungen zur visuellen Datenanalyse für die FuE-Abteilungen pharmazeutischer Unternehmen. Die Software soll dazu beitragen, die Prozesse in der Wirkstoffforschung zielgerichteter zu gestalten und damit die Entwicklung neuer Medikamente effizienter zu machen. Die Software unterstützt dabei insbesondere die Entscheidungsfindung auf Grundlage der erfassten Datensammlungen.

Nach Einschätzung von wissenschaftlich am Projekt beteiligten Personen an der Medizinischen Universität Graz hat GenOptikum auch bei diesem Partner sehr gute fachliche Resultate geliefert, die in mehreren nationalen und EU-Projekten weitergeführt wurden. Im Institut für Pathologie haben sich die Forschungsschwerpunkte in den letzten zehn Jahren zwar weiterentwickelt. Es hätten sich aber beispielsweise auch für die Entwicklung von Lösungen in der Digitalen Pathologie zahlreiche, mit GenOptikum-Ergebnissen in Zusammenhang stehende Forschungsfragen ergeben ( $z$. B. für die automatisierte Auswertung von Bibliotheken histologischer Schnitten), die heute neue Ansätze von Artifical Intelligence und Machine Learning für die Interpretation medizinischer Informationen erfordern.

Das am Projekt beteiligte Unternehmen Oridis Biomed war vor allem für die Formulierung von Anforderungen an die Visualisierung von genetischen Informationen zuständig. Eine Nutzung der Ergebnisse ist für dieses Unternehmen nicht mehr möglich, da es bereits im Jahr 2012 liquidiert wurde. 
Abbildung 34 Anteil der Unternehmen mit PATSTAT-Eintrag unter den Antrag stellenden Unternehmen in den BMVIT IKT-Programmen im Zeitraum 2002 bis 2016, nach Programmen

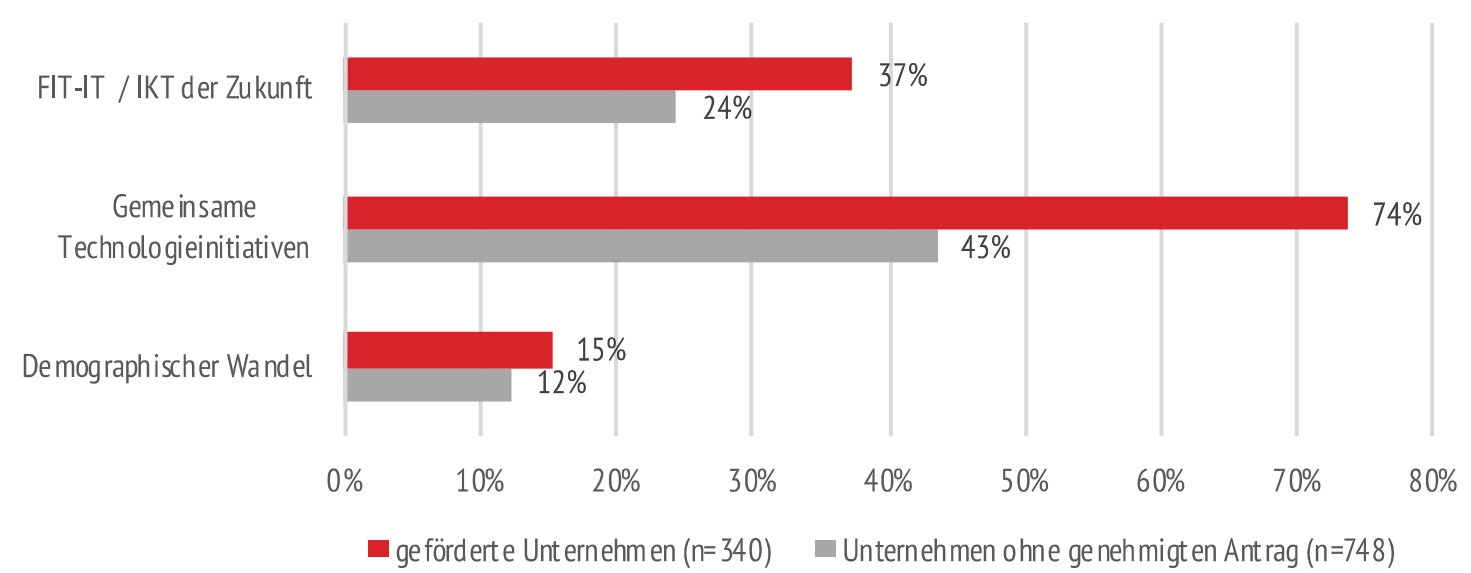

Quelle: FFG, PATSTAT, Auswertung inspire research

Die Daten zeigen, dass der überwiegende Anteil der Patente der Programmteilnehmer/innen von Unternehmen angemeldet wurde. Der Anteil der Forschungseinrichtungen unter den insgesamt angemeldeten Patenten durch Antragsteller/innen in den BMVIT IKT-Programmen liegt im Auswertungszeitraum bei rund $10 \%$.

Abbildung 35 Anzahl der PATSTAT erfassten Patentanmeldungen von Antragstellern in den BMVIT IKT-Programmen 2002 bis 2014

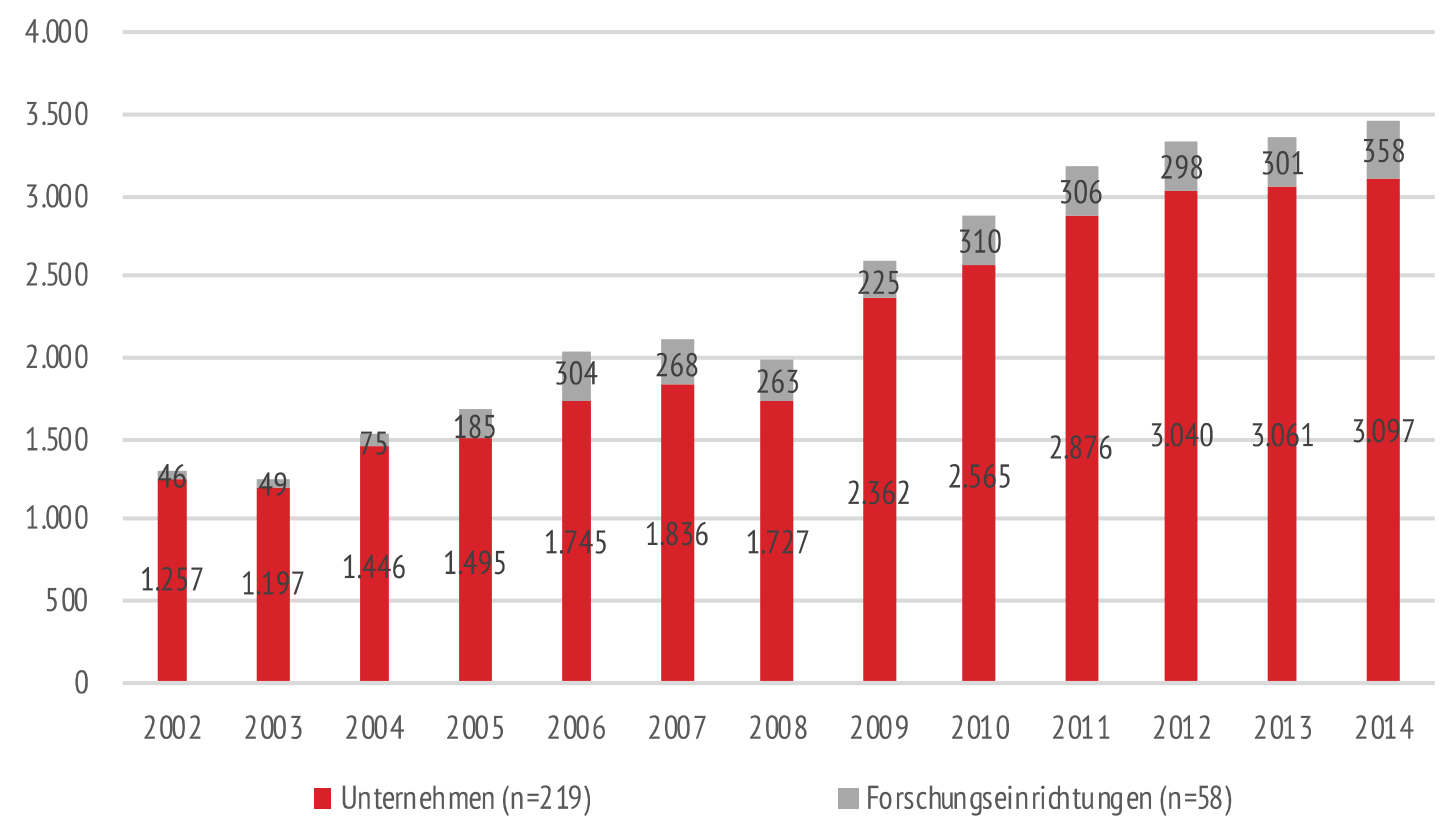

Quelle: FFG, PATSTAT; Auswertung und Darstellung inspire research

In einem weiteren Schritt haben wir geprüft, ob in den Patentanmeldungen der Einrichtungen, die an Anträgen in den BMVIT IKT-Programmen beteiligt waren, auch technische Ansprechpersonen der beteiligten wissenschaftlichen Einrichtungen in den BMVIT IKT-Programmen als Anmelder oder Erfinder genannt sind, d. h. ob es zu gemeinsamen Patentanträgen zwischen technischen Ansprechpersonen und Einrichtungen in den BMVIT IKT-Programmen kam. Unberücksichtigt blieb dabei vorerst, ob es sich beim Anmelder des Patents um die wissenschaftliche Einrichtung der technischen Ansprechpersonen handelte oder um ein am Programm beteiligtes Unternehmen. 
Für etwas mehr als ein Drittel (35\%) der technischen Ansprechpersonen geförderter Projekte der Gemeinsamen Technologieinitiativen finden sich PATSTAT-Einträge, die von an den BMVIT IKT-Programmen beteiligten Einrichtungen angemeldet wurden. Unter den technischen Ansprechpersonen geförderter Projekte in den FIT-IT Programmlinien / IKT der Zukunft beträgt dieser Anteil ein Viertel (25\%). Das geringste Ausmaß an gemeinsamen Patentanträgen zwischen technischen Ansprechpersonen der Forschungseinrichtungen und Einrichtungen in den BMVIT IKT-Programmen zeigt sich unter den technischen Ansprechpersonen in Projekten zum Demographischer Wandel (13\%).

Abbildung 36 Anteil der technischen Ansprechpersonen der wissenschaftlichen Partner, die an Patenten von Einrichtungen, die im Zeitraum 2002 bis 2016 in den BMVIT IKT-Programmen Anträge gestellt haben, angemeldet wurden

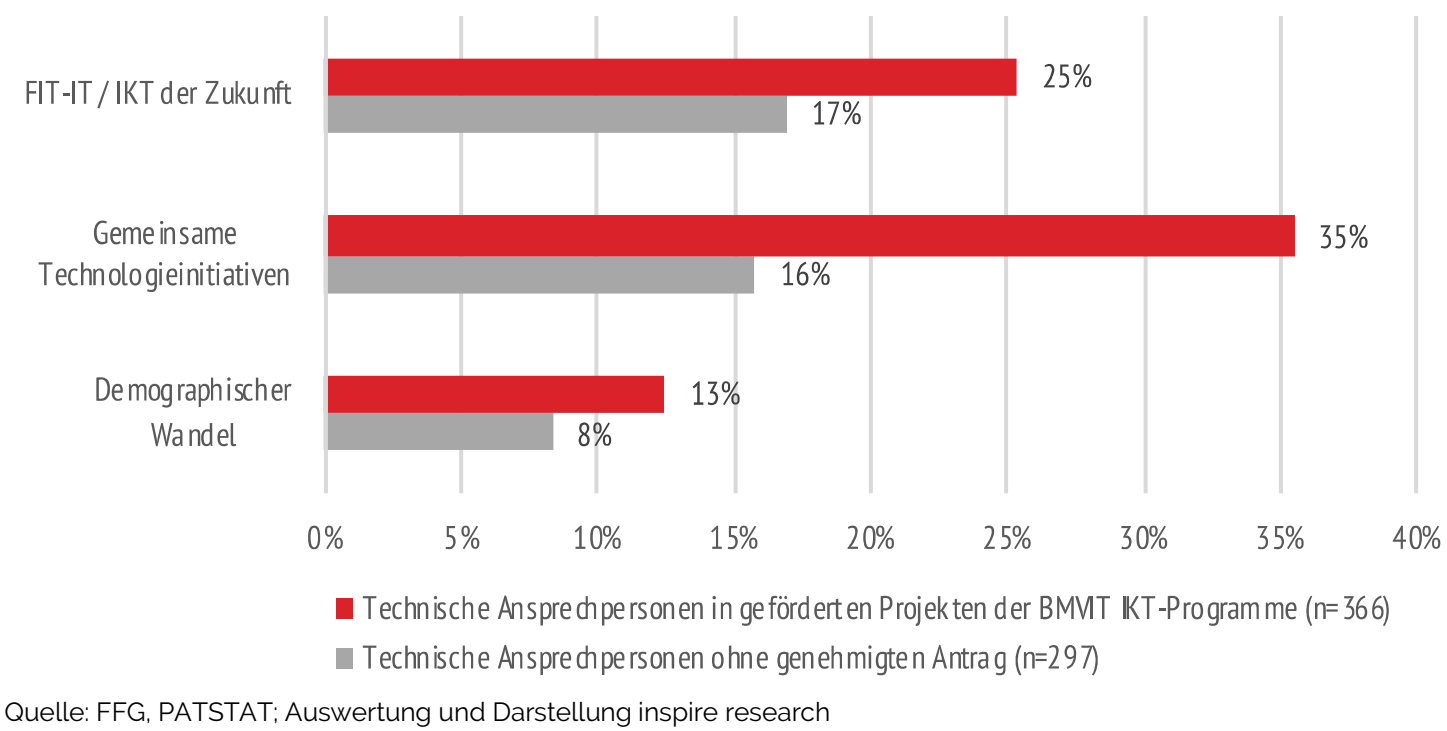

Etwas weniger als zwei Drittel (62 \%) der technischen Ansprechpersonen der wissenschaftlichen Partner, die in Patenten von Einreichern in den BMVIT IKT-Programmen erfasst sind, sind in Patentanmeldungen der eigenen Einrichtung genannt, etwas mehr als ein Drittel (34\%) in Patentanmeldungen von beteiligten Unternehmen. Ein Viertel (25\%) der technischen Ansprechpersonen der wissenschaftlichen Partner ist in Patentanträgen genannt, die von anderen an den Programmen beteiligten wissenschaftlichen Partnern angemeldet wurden.

Nennung technischer Ansprechpersonen von wissenschaftlichen Einrichtungen in Patentanmeldungen der Unternehmen

Bei den Patentanmeldungen von an den BMVIT IKT-Programmen beteiligten Unternehmen, in denen auch technische Ansprechpersonen einer Forschungseinrichtung aufscheinen, handelt es sich nur in wenigen Fällen um jene Personen, mit denen gemeinsam der Antrag gestellt wurde. Insgesamt 13 von 86 technischen Ansprechpersonen mit Patenteinträgen (15\%) sind in Patentanträgen von Unternehmen genannt, mit denen auch gemeinsame Projekte in den BMVIT IKT-Programmen durchgeführt wurden. In der Gruppe der technischen Ansprechpersonen mit Patenteinträgen, die kein Projekt bewilligt erhielten, sind es 5 von 42 Personen (12\%), die in Patentanträgen von Unternehmen genannt werden, mit denen gemeinsam ein Projekt in den BMVIT IKT-Programmen beantragt wurde. 


\section{Anteil der Patentanmeldungen in IKT-Technologiefeldern}

Die PATSTAT-Daten zeigen auch, dass in den IKT-Programmen Unternehmen gefördert wurden, deren Patentanmeldungen in deutlich stärkerem Ausmaß als bei nicht-geförderten Unternehmen in IKT-Technologiefelder fallen. Unter den geförderten Unternehmen nahm der Anteil der Patentanmeldungen in IKT-Technologiefeldern zwischen 2002 und 2014 von 11 \% auf 30 \% deutlich zu, während unter den nicht-geförderten Unternehmen der Anteil von Patentanmeldungen in IKT-Technologiefeldern in diesem Zeitraum tendenziell abnahm.

Die Auswertung der PATSTAT-Daten gibt auch einen Hinweis auf die zunehmende Internationalisierung der Patentanmeldungen von österreichischen Unternehmen in IKT-Technologiefeldern, die in den BMVIT IKT-Programmen aktiv waren. Während bei den im Jahr 2002 von Unternehmen angemeldeten Patenten in IKT-Technologiefeldern noch mehr als ein Drittel (35\%) in Österreich angemeldet wurden, lag dieser Anteil im Jahr 2014 nur mehr bei $8 \%$.

Abbildung 37 Anteil der Patenanmeldungen in IKT-Technologiefeldern* von geförderten und antragstellenden, nicht-geförderten Unternehmen in den BMVIT IKT-Programmen 2002 bis 2014

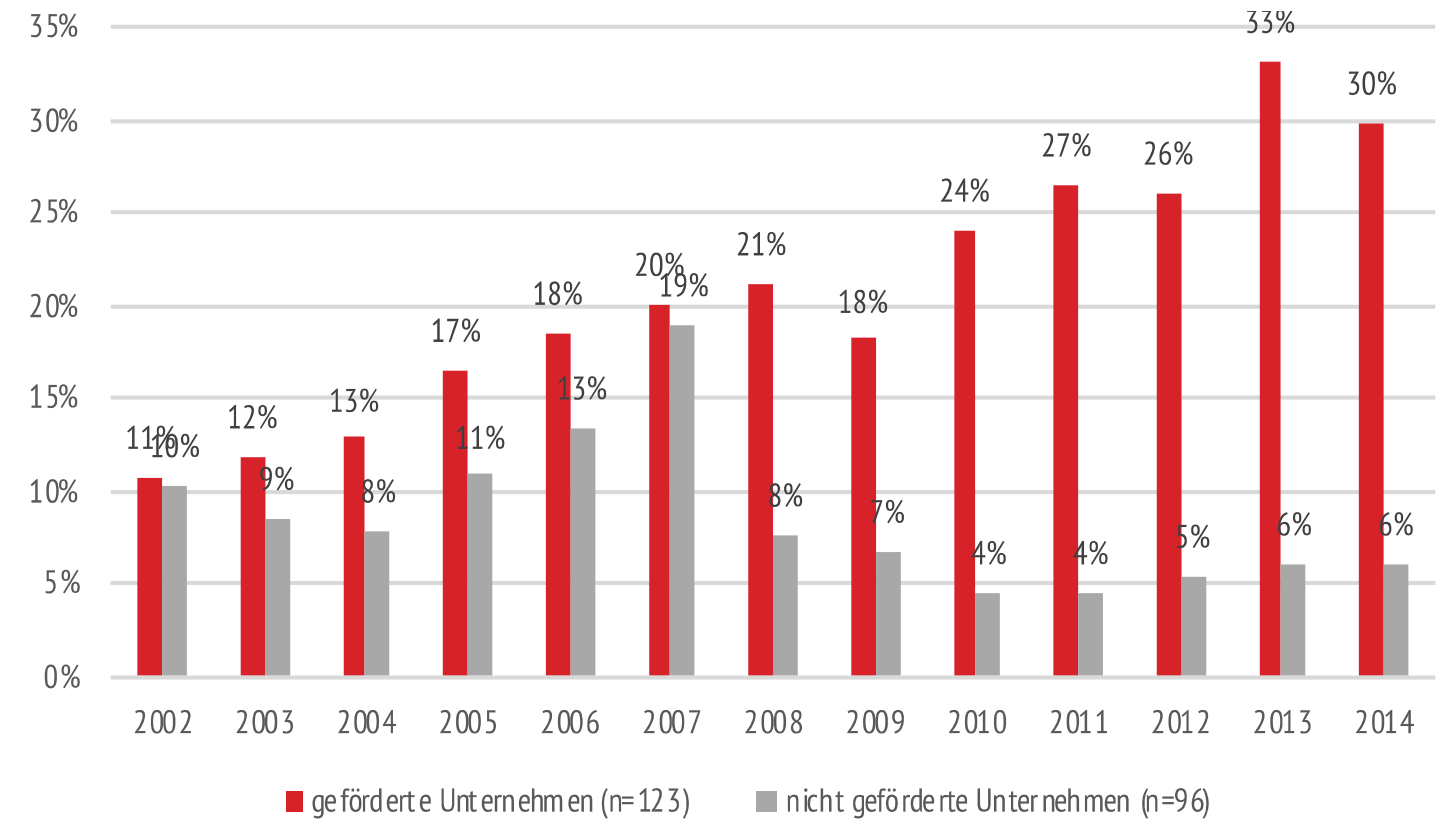

*IKT-Technologiefelder: Audio-visual technology; Telecommunications; Digital communication; Basic communication processes; Computer technology; IT methods for management; Semiconductors

Quelle: FFG, PATSTAT; Auswertung und Darstellung inspire research

Dieser Trend zu Internationalisierung ist bei Patentanmeldungen in IKT-Technologiefeldern deutlich stärker ausgeprägt als bei Patentanmeldungen in anderen Technologiefeldern.

Auch wenn eine direkte Zuordnung der Patente der Förderempfänger in den BMVIT IKT-Programmen zu den geförderten Projekten nicht möglich ist, zeigen die Patentanalysen doch eine deutlich aktivere Patentierungstätigkeit der an den Programmen beteiligten Unternehmen im Vergleich zur Gruppe der nicht-geförderten Unternehmen. Zwar gaben nur $16 \%$ der Unternehmen, die bereits bis Ende 2016 Projekte im Programm IKT der Zukunft abgeschlossenen hatten, in der Befragung an, Ergebnisse (bereits) patentiert zu haben. Gleichzeitig finden wir aber fast ein Drittel (31\%) der Antrag stellenden Unternehmen in den BMVIT-Programmen, die seit 2002 Erfindungen patentiert haben. 


\section{Box 6: Langfristige Wirkungen der FIT-IT Förderung: SPECTACLES (Wearable Autonomous Displays)}

Ziel des Projekts SPECTACLES war es, Standard Software- und Hardwarekomponenten in einer Brille zu integrieren und die dazu notwendigen eingebetteten Rechner- und Kommunikationselemente zu definieren und zu entwickeln. Neben einer integrierten Energieversorgung umfasste die Plattform ein modulares System aus Sensoren, Sprache-zu-Textund Text-zu-Sprache-Komponenten, Standort- und Positionierungselementen und Mikrodisplays. Als Open Source Lösung sollten die Ergebnisse für beliebige Anwendungsfälle von Wearable Devices genutzt werden können.

Das Projekt wurde vom Institut für Pervasive Computing an der Johannes Kepler Universität Linz (JKU Linz) in der 4. Ausschreibung der FIT-IT Programmlinie Embedded Systems eingereicht. Am Projekt waren der Fachbereich Computerwissenschaften an der Universität Salzburg sowie als Unternehmenspartner der Linzer Brillenhersteller Silhouette International beteiligt.

Das Unternehmen wirkte vor allem bei der Festlegung der funktionalen, wirtschaftlichen und ästhetischen Anforderungen an die entwickelten Lösungen mit. Das Unternehmen lieferte hierzu wichtige Hinweise für die Gestaltung der Produkte unter dem Gesichtspunkt der Kundenakzeptanz. Silhouette konnte die Ergebnisse des Projekts später jedoch nicht für die Entwicklung eigener Produkte nutzen. Die Jahre nach Abschluss des Projekts fielen im Unternehmen mit ernsten wirtschaftlichen Herausforderungen im Zusammenhang mit Produktpiraterie und Urheberrechtsverletzungen seitens internationaler Konkurrenten zusammen, die gerichtlich mit großem Aufwand verfolgt wurden.

Im Projekt kam es zu einer Kooperation mit führenden internationalen Forschungsgruppen im Bereich Wearable Computing, wobei insbesondere die Zusammenarbeit mit der University of Central California und dem Massachusetts Institute of Technology (MIT) für die Arbeiten von großer Bedeutung war. Mitglieder der Forschungsteams bei den amerikanischen Projektpartnern waren später maßgeblich an der Entwicklung von Google Glass beteiligt. Es waren auch die Partner an den amerikanischen Universitäten, die Projektergebnisse schließlich patentierten.

An der JKU Linz stand hingegen die wissenschaftliche Verwertung im Vordergrund. Aus den Ergebnissen des Projekts sind zahlreiche Konferenzbeiträge und wissenschaftliche Zeitschriftenartikel entstanden. In den Folgejahren engagierte sich das Team der JKU Linz aktiv an der Organisation von internationalen wissenschaftlichen Konferenzen, um die erzielten Forschungsergebnisse weiterzutragen und organisierte dazu spezielle Workshops. Auch mehrere EU-Projekte wurden in der Folge durchgeführt. So arbeitete das Institut an der JKU Linz nach Abschluss von Spectacles gemeinsam mit der ETH Zürich, der EFP Lausanne und der Universität Passau an einem FET Open Projekt zum Thema "Activity and Context Recognition with Opportunistic Sensor Configurations" mit.

Nicht zuletzt konnte eine Promotion mit Unterstützung des Spectacles-Projekts durchgeführt werden. Allerdings reichte die Laufzeit des Projekts nicht aus, um dem Doktoranden die Durchführung der gesamten Dissertation innerhalb des Projekts zu ermöglichen.

Spectacles stellte für das Institut für Pervasive Computing den Startpunkt für eine bis heute andauernde Projekttrajektorie dar. Mit dem Projekt konnten Fortschritte zu Fragen von Activity Recognition und Context Recognition erzielt werden, die aktuell am Institut vor allem für Forschungsfragen zum Machine Learning / Artificial Intelligence genutzt werden. 
Abbildung 38 Anteil der in PATSTAT erfassten Patentanmeldungen von Unternehmen in den BMVIT IKT-Programmen, die in Österreich angemeldet wurden nach Technologiefeldern* 2002 - 2014

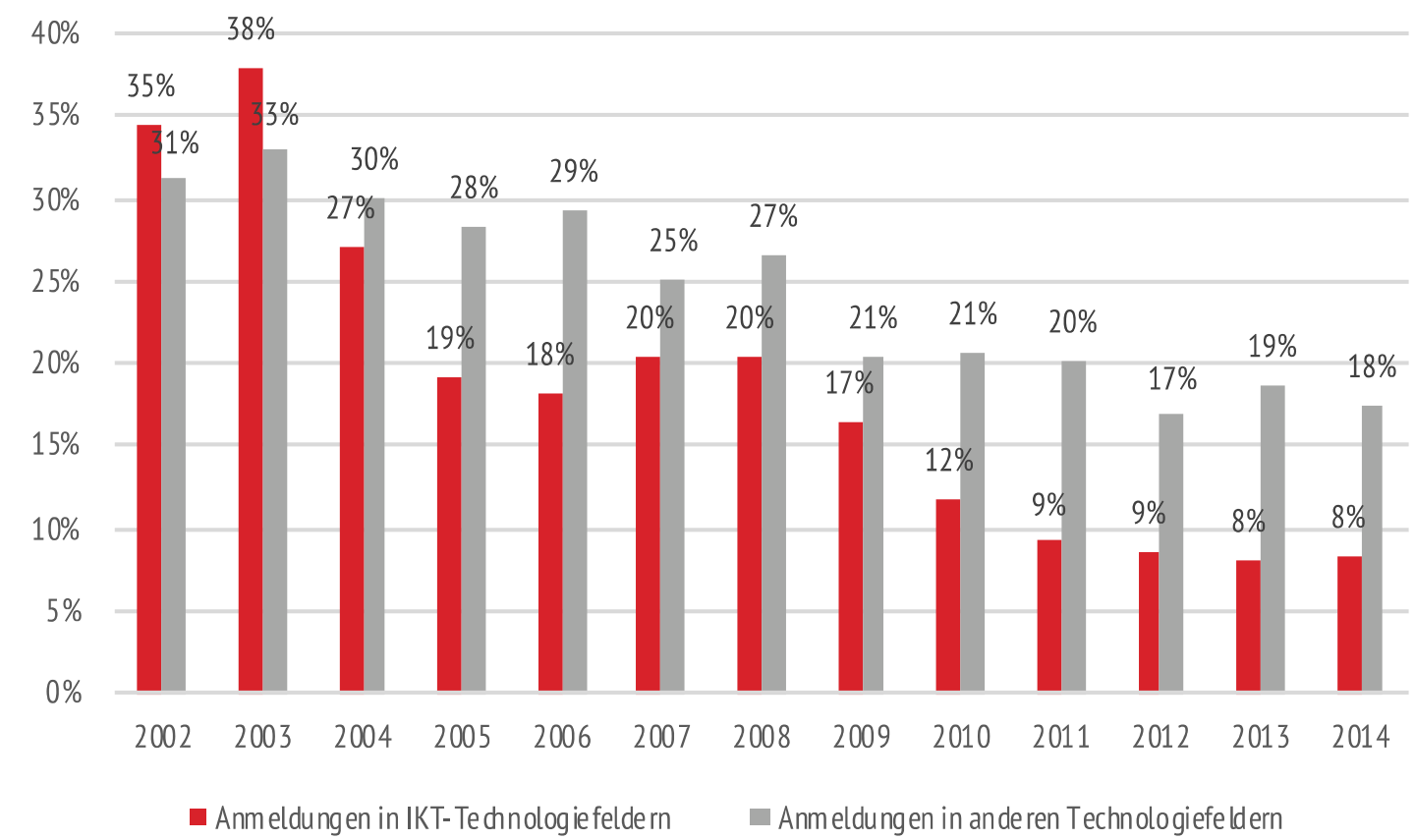

*IKT-Technologiefelder: Audio-visual technology; Telecommunications; Digital communication; Basic communication processes; Computer technology; IT methods for management; Semiconductors:

Quelle: FFG, PATSTAT; Auswertung und Darstellung inspire research

Sowohl die Anzahl als auch der Anteil an Patentanmeldungen in IKT-Technologiefeldern hat seit Start des Impulsprogramms FIT-IT im Jahr 2002 zudem stark zugenommen. Außerdem weisen die Auswertungen der PATSTAT-Daten auf eine deutliche Internationalisierung der Patentanmeldungen österreichischer Unternehmen hin, die in den BMVIT IKT-Programmen gefördert wurden.

\subsection{Entwicklung von FuE-Ausgaben und FuE-Beschäftigung}

Als Teil der Evaluierung wurde eine Sonderauswertung der FuE-Erhebungen von Statistik Austria durchgeführt. Dazu wurde Statistik Austria eine Liste der Firmenbuchnummern jener Unternehmen übermittelt, die jeweils in den Vierjahresperioden 2002 bis 2005, 2006 bis 2009 sowie 2010 bis 2013 in den BMVIT IKT-Programmen einen Förderantrag gestellt hatten.

Die Zuordnung zur den Perioden erfolgte nach der tatsächlichen Laufzeit (bei geförderten Unternehmen) bzw. der geplanten Laufzeit (bei nicht-geförderten Unternehmen) der Projekte gemäß FFG-Datenbank. Das heißt, wurde ein Unternehmen für ein von 1. Oktober 2005 bis 30. September 2008 laufendes Projekt gefördert, wurde es für die Periode 2002 bis 2005 und für die Periode 2006 bis 2009 der Gruppe der geförderten Unternehmen geordnet. Reichte beispielsweise dieses Unternehmen zwei weitere, nicht genehmigte Anträge mit einer vorgesehenen Projektlaufzeit von 1. Juli 2007 bis 30. Juni 2010 bzw. von 1. Jänner 2010 bis 31. Dezember 2012 wurde das Unternehmen für die Periode 2010 bis 2013 der Gruppe der nicht-geförderten Unternehmen zugeordnet.

Das Team von Statistik Austria identifizierte anhand der Firmenbuchnummern jene Unternehmen, die für die Erhebungsjahre 2004, 2009 und 2013 auch in der FuE-Erhebung erfasst wurden. Für diese Unternehmen wurden getrennt nach geförderten Unternehmen und nichtgeförderten Unternehmen in aggregierter Form Vergleiche zur Höhe und Entwicklung der 
internen FuE-Ausgaben, der Finanzierung der internen FuE-Ausgaben sowie der FuE-Beschäftigten vorgenommen.

Die Vergleiche wurden sowohl zwischen der Gruppe der geförderten und der nicht-geförderten Unternehmen in den BMVIT-IKT-Programmen durchgeführt, als auch zwischen den geförderten Unternehmen und der Grundgesamtheit aller in der FuE-Erhebung erfassten Unternehmen im firmeneigenen Bereich (d. h. ohne Berücksichtigung des kooperativen Sektors, der vor allem die außeruniversitären Forschungseinrichtungen umfasst).

Abbildung 39 Vergleich der Entwicklung der internen FuE-Ausgaben sowie der Zahl der FuE-Beschäftigten zwischen den geförderten und den einreichenden, nicht-geförderten Unternehmen mit (geplanten) FIT-IT Projekten in den Jahren 2002 bis 2005

FIT-IT geförderte Unternehmen (2002-2005)

gesunkene

gestiegene

\begin{tabular}{ccl}
2 & 16 & FuE-Ausgaben \\
\hline 3 & 15 & Beschäftigtenzahlen
\end{tabular}

nicht FIT-IT geförderte Unternehmen (2002-2005)

gesunkene

\begin{tabular}{rrr}
11 & 7 \\
\hline & 10 & 8
\end{tabular}

gestiegene

FuE-Ausgaben

Beschäftigtenzahlen

Quelle: FFG, Statistik Austria, Darstellung inspire research

Die Sonderauswertung der FuE-Erhebungen für die Jahr 2004 und 2009 zeigt, dass Unternehmen, die in den Jahren 2002 bis 2005 in den BMVIT IKT-Programmen gefördert wurden, sich hinsichtlich ihrer internen FuE-Ausgaben und der Zahl der FuE-Beschäftigten tendenziell besser entwickelt haben, als einreichende, nicht-geförderte Unternehmen.

Abbildung 40 Veränderung der internen FuE-Ausgaben in \% zwischen den Erhebungsjahren 2004 und 2009 unter den geförderten und den einreichenden, nicht-geförderten Unternehmen mit (geplanten) FIT-IT Projekten in den Jahren 2002 bis 2005 sowie Vergleich mit der Gesamtheit der Unternehmen in der FuE-Statistik (firmeneigener Bereich)

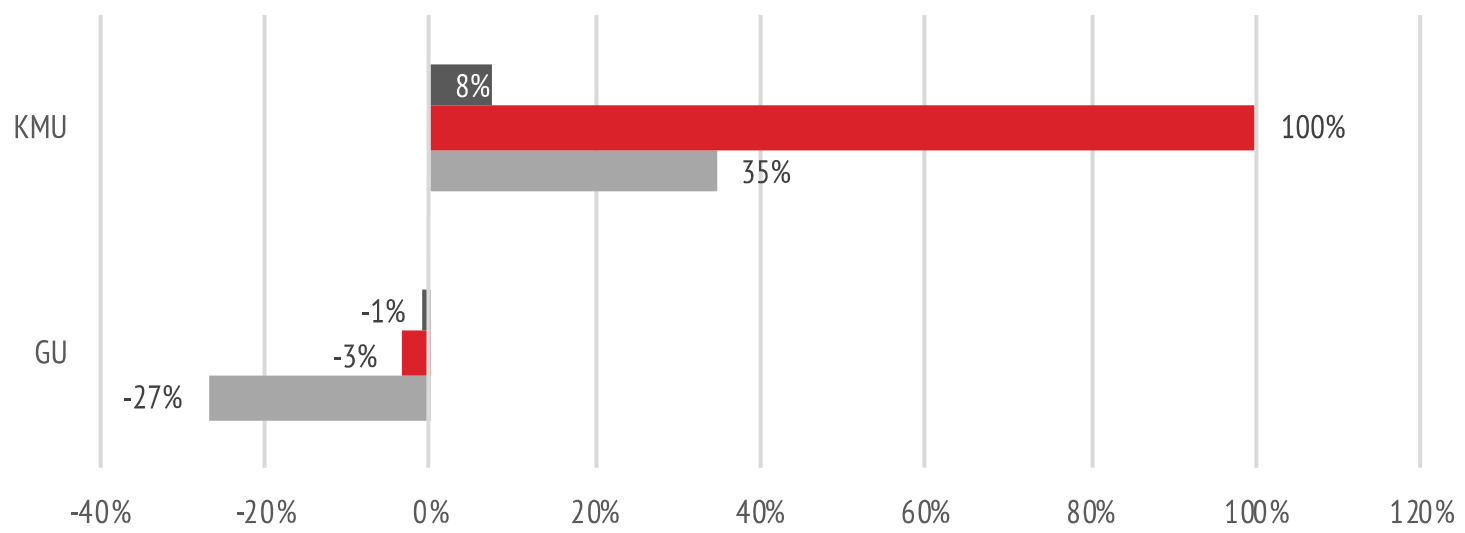

ש alle Unternehmen ( $n=1.244)$ — FIT-IT geför dert $(n=18)$ — FIT-IT Antrag, nicht geför dert $(n=18)$

Quelle: FFG, Statistik Austria, Darstellung inspire research

Allerdings zeigt sich auch, dass auf Basis eines Vergleichs mit der Grundgesamtheit aller Unternehmen in der FuE-Erhebung eine bessere Entwicklung nur für die Gruppe der KMU zu 
sehen ist. Die in den Jahren 2002 bis 2005 im Programm FIT-IT geförderten Großunternehmen konnten sich hinsichtlich ihrer internen FuE-Ausgaben (ohne Berücksichtigung der öffentlichen Finanzierungsanteile durch direkte Subventionen und den Beitrag der Forschungsprämie) nicht besser entwickeln als die Grundgesamtheit der in den FuE-Erhebungen der Jahre 2004 und 2009 erfassten Großunternehmen.

Ein ähnliches Bild zeigt sich für die Entwicklung der FuE-Beschäftigten der in den Jahren 2004 und 2009 forschungsaktiven Unternehmen. Auch hier ist eine Zunahme nur in der Gruppe der KMU festzustellen.

Abbildung 41 Veränderung der Anzahl der FuE-Beschäftigten in \% zwischen den Erhebungsjahren 2004 und 2009 unter den geförderten und den einreichenden, nicht-geförderten Unternehmen mit (geplanten) FIT-IT Projekten in den Jahren 2002 bis 2005 sowie Vergleich mit der Gesamtheit der Unternehmen in der FuE-Statistik (firmeneigener Bereich)

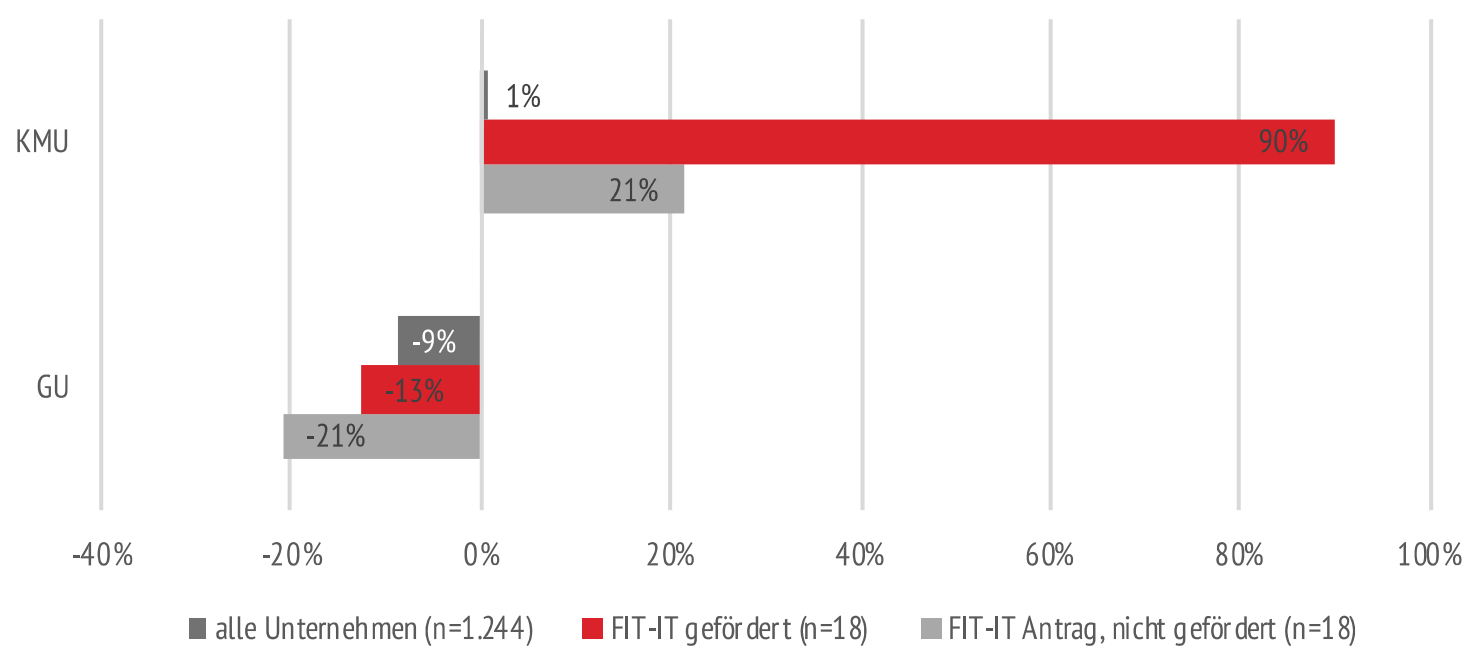

Quelle: FFG, Statistik Austria, Darstellung inspire research

Diese Ergebnisse deuten tendenziell auf eine geringe Effektivität der Förderung im Bereich der Großunternehmen hin. Geförderte und nicht-geförderte KMU steigerten hingegen ihre internen FuE-Ausgaben (ohne öffentlichen Förderanteil) in größerem Ausmaß als die Grundgesamtheit der Unternehmen, die in den FuE-Statistiken der Jahre 2004 und 2009 erfasst sind, wobei die geförderten KMU ihre FuE-Ausgaben besonders deutlich erhöhen konnten.

Für den Förderzeitraum 2006 bis 2009 lässt sich kaum mehr ein Zusammenhang zwischen Förderung in den IKT-Programmen und einer stärker positiven Entwicklung der internen FuEAusgaben bzw. der FuE-Beschäftigung feststellen. Zwar nahmen bei einem größeren Anteil der geförderten Unternehmen die internen FuE- Ausgaben weiter zu. Bei der Zahl der FuEBeschäftigten war das Verhältnis jedoch umgekehrt. 
Abbildung 42 Vergleich der Entwicklung der internen FuE-Ausgaben sowie der Zahl der FuE-Beschäftigten zwischen den geförderten und den einreichenden, nicht-geförderten Unternehmen mit (geplanten) FIT-IT Projekten in den Jahren 2006 bis 2009

FIT-IT geförderte Unternehmen (2006-2009)

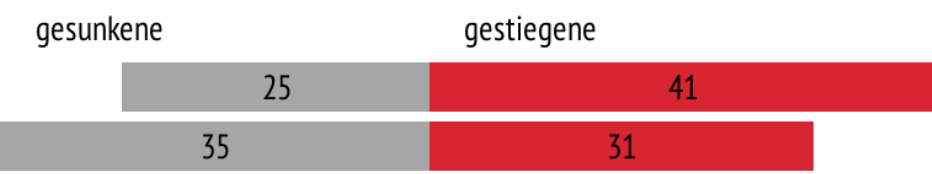

FuE-Ausgaben

Beschäftigtenzahlen

nicht FIT-IT geförderte Unternehmen (2006-2009)

gesunkene

gestiegene

\begin{tabular}{|r|r|}
\hline 24 & 17 \\
\hline 17 & 24 \\
\hline
\end{tabular}

FuE-Ausgaben

Beschäftigtenzahlen

Quelle: FFG, Statistik Austria, Darstellung inspire research

Differenzierung wir die Ergebnisse wieder nach Unternehmensgröße, so haben sich die internen FuE-Ausgaben (ohne Subventionen) bei den geförderten Großunternehmen zwischen 2009 und 2013 nur unwesentlich dynamischer entwickelt als in der Grundgesamtheit aller in den Jahren 2009 und 2013 erfassten Unternehmen der FuE-Erhebung.

Abbildung 43 Veränderung der internen FuE-Ausgaben in \% zwischen den Erhebungsjahren 2009 und 2013 unter den geförderten und den einreichenden, nicht-geförderten Unternehmen mit (geplanten) FIT-IT Projekten in den Jahren 2006 bis 2009 sowie Vergleich mit der Gesamtheit der Unternehmen in der FuE-Statistik (firmeneigener Bereich)

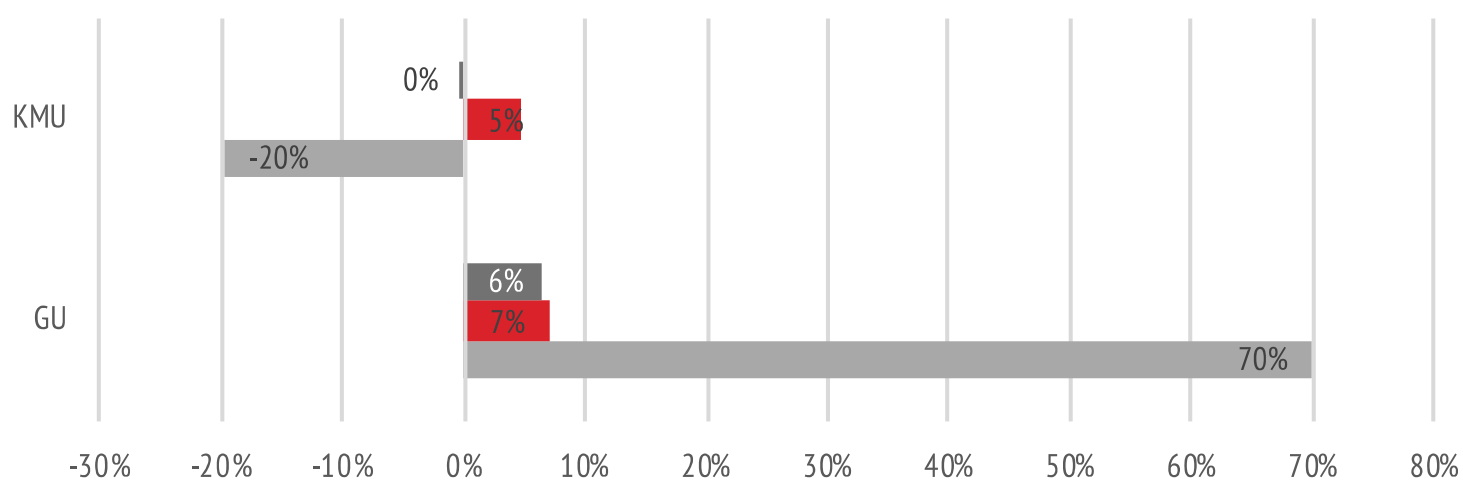

alle Unternehmen $(n=1.860) \quad \square$ FIT-IT g eför dert $(n=66) \quad$ FIT -IT Antrag, nicht g eför dert $(n=41)$

Quelle: FFG, Statistik Austria, Darstellung inspire research

Bezogen auf die Zahl der FuE-Beschäftigten in den geförderten Unternehmen lässt sich ebenfalls keine bessere Entwicklung der FIT-IT geförderten Unternehmen im Vergleich zur Grundgesamtheit aller in den Jahren 2009 und 2013 erfassten Unternehmen der FuE-Erhebung feststellen. 
Abbildung 44 Veränderung der Zahl der FuE-Beschäftigten in \% zwischen den Erhebungsjahren 2009 und 2013 unter den geförderten und den einreichenden, nicht-geförderten Unternehmen mit (geplanten) FIT-IT Projekten in den Jahren 2006 bis 2009 sowie Vergleich mit der Gesamtheit der Unternehmen in der FuE-Statistik (firmeneigener Bereich)

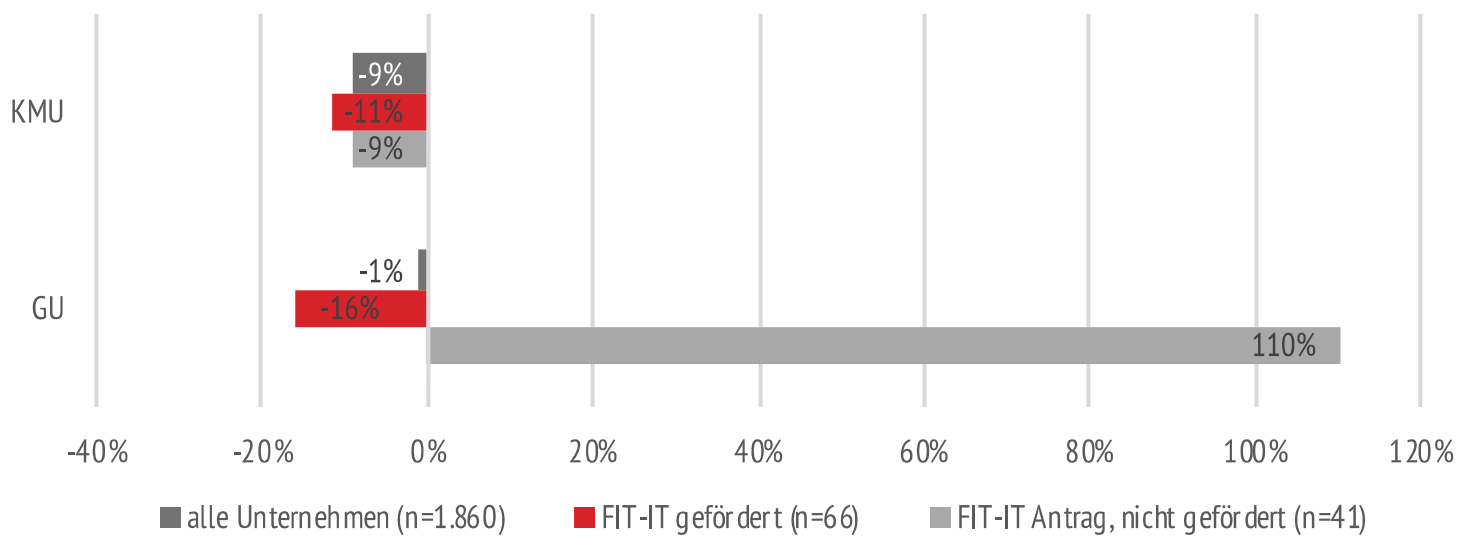

Quelle: FFG, Statistik Austria, Darstellung inspire research

Die Ergebnisse der Sonderauswertung der FuE-Erhebungen der Jahre 2004, 2009 und 2013 zeigen für Unternehmen, die im FIT-IT-Programm Projekte mit Laufzeitbeginn bis Ende 2009 eingereicht hatten, nur für die Gruppe der KMU in der ersten Programmphase eine positive Korrelation zwischen der Projektförderung im FIT-IT Programm und der Entwicklung der internen FuE-Ausgaben sowie der Entwicklung der Zahl an FuE-Beschäftigten. Für große Unternehmen kann hingegen kein Zusammenhang zwischen Förderung und einer stärker positiven Entwicklung der interbetrieblichen FuE-Kennzahlen Ausgaben und Beschäftigung im Vergleich zu allen in den FuE-Erhebungen erfassten Unternehmen festgestellt werden. Zu beachten ist dabei, dass die Unternehmen auch aus zahlreichen anderen Quellen öffentliche FuE-Finanzierungen für ihre internen FuE-Ausgaben erhielten. Ein nicht feststellbarer Zusammenhang zwischen Förderung und Entwicklung der genannten Kennzahlen muss also nicht unbedingt auf eine fehlende Wirkung der FIT-IT Förderung hindeuten. 


\section{Diskussion der Ergebnisse}

In diesem Abschnitt fassen wir die Ergebnisse der Evaluierung zusammen und diskutieren sie im Hinblick auf die gestellten Evaluierungsfragen.

\subsection{Konnten die Ziele des Impulsprogramms FIT-IT erreicht werden?}

Die Erhebungen und Auswertungen in der ex-post Evaluierung des Impulsprogramms FIT-IT zeigen, dass es dem Programm in den Jahren 2002 bis 2012 insgesamt sehr gut gelungen ist, die gesetzten Ziele zu erreichen. Ein sehr hoher Anteil von 81 \% der ehemals beteiligten wissenschaftlichen Partner und $67 \%$ der ehemals beteiligten Unternehmen gaben in der Befragung an, die FIT-IT Projekte hätten Nachwirkungen über die Projektlaufzeit hinaus gehabt, die auch heute noch - und damit viele Jahre nach Beendigung der Projekte - von Bedeutung für ihre Einrichtung seien. Hervorgehoben wurden insbesondere die Beiträge der FIT-IT Projekte zur Qualifizierung der Mitarbeiter/innen und zum internen Know-how-Aufbau sowie zur Verbesserung der Netzwerke und Kooperationen für die Forschungszusammenarbeit im IKTBereich. Die FIT-IT Förderung hat nach Einschätzung der Programmteilnehmer/innen insbesondere in den ersten Jahren der Programmdurchführung maßgeblich zur Stärkung der Leistungs- und Wettbewerbsfähigkeit der österreichischen IKT-Forschung und der Kooperationen zwischen Wirtschaft und Wissenschaft beigetragen.

Die Hinweise der FIT-IT-Programmteilnehmer/innen in der Evaluierung lassen auch den Schluss zu, dass mit dem Programm die Sichtbarkeit österreichischer Forschungsgruppen und Unternehmen im internationalen Kontext - insbesondere in den europäischen Programmen - durch FIT-IT deutlich verbessert werden konnte. Die Programmteilnehmer/innen berichteten über zahlreiche Fälle, in denen es zur Fortführung bzw. Weiterentwicklung der nationalen FuE-Projekte in europäischen Forschungskooperationen gekommen sei. Hierzu haben nicht nur die kooperativen FuE-Projekte, sondern auch die Stimulierungsprojekte einen Beitrag leisten können.

Mit dem FIT-IT Programm ist es gelungen, eine große Anzahl von Akteuren in Wirtschaft und Wissenschaft zu mobilisieren. Über die Laufzeit des Impulsprogramms FIT-IT haben sich praktisch alle relevanten wissenschaftlichen Einrichtungen mit IKT-Schwerpunkten in Österreich und mehr als 400 unterschiedliche Unternehmen an den Ausschreibungen beteiligt. Mehr als 300 Unternehmen wurden in den bewilligten FIT-IT Projekten auch gefördert. Über die Programmlaufzeit von FIT-IT wurden rund 95 Mio. Euro an Fördermittel vergeben, wobei mehr als 40 \% der Förderungen den Hochschulen zugute kam. Damit leistete das Impulsprogramm einen wichtigen Beitrag, wissenschaftlich anspruchsvolle, kooperative Forschungsprojekte durchzuführen und damit junge Wissenschaftler/innen für Wissenschaft und Wirtschaft auszubilden und zu qualifizieren. Innerhalb der Projektdauer der geförderten FIT-ITProjekte - mehr als $70 \%$ der geförderten Projekte hatten eine Laufzeit zwischen zwei und drei Jahren - war es jedoch Doktorand/inn/en nur in Ausnahmefällen möglich, ohne weitere Finanzierungsquellen oder Förderprojekte ihre Promotionen auch abzuschließen.

Die bibliometrischen Auswertungen zeigen eine intensive Publikationstätigkeit der wissenschaftlichen Partner, aber auch der beteiligten Unternehmen, während und nach der Laufzeit der FIT-IT Projekte. Selbst unter der konservativen Annahme, dass nur ein Viertel des in Scopus erfassten Publikationsoutputs der technischen Ansprechpersonen bei den wissenschaftlichen Partnern, die während der Laufzeit des Projekts veröffentlicht wurden, dem FIT-IT Projekt zugerechnet werden können, errechnen sich noch immer vier wissenschaftliche Publikationen je beteiligtem wissenschaftlichen Partner als Publikationsleistung aus dem Projekt. 
Nach Einschätzung der Programmteilnehmer/innen und anderer Personen, die in die Programmkonzeption und Programmabwicklung einbezogen waren, ist es dem FIT-IT-Programm zwar nicht unbedingt gelungen, dem Programmanspruch entsprechend auf breiter Linie radikal neue Informationstechnologie bis zum funktionsnachweisenden Forschungsprototyp in Österreich zu entwickeln. Gleichzeitig zeigen aber die Fallstudien zu den geförderten FIT-IT Projekten doch sehr deutlich einen langfristig sehr positiven Beitrag der Förderung zur nachhaltigen wissenschaftlichen und wirtschaftlichen Verwertung der Forschungsergebnisse. Aufgrund des Designs, der Orientierung und der Durchführung des Impulsprogramms FIT-IT kann jedenfalls von einer hohen Additionalität der Förderung, insbesondere im wissenschaftlichen Bereich, ausgegangen werden.

Die an FIT-IT beteiligten Unternehmen bewerten die wirtschaftlichen Erfolge und den Beitrag zur Steigerung ihrer Wettbewerbsfähigkeit durchwegs positiv. Fast die Hälfte (45\%) der an FIT-IT Projekten beteiligten Unternehmen berichteten über einen besseren Zugang zu Kund/innen und fast ein Viertel (24\%) über unmittelbare Umsatzsteigerungen bzw. verringerte Kosten aufgrund der Durchführung der FIT-IT Projekte. Der Hauptnutzen der Beteiligung an den FIT-IT Projekten wird aber im Aufbau von internem Know-how, der Verbesserung der Zusammenarbeit mit Forschungseinrichtungen und Unternehmen sowie in der Schaffung der Grundlage für die Weiterentwicklung der Ergebnisse in FuE-Projekten und Kundenprojekten gesehen.

Die Auswertung der PATSTAT-Datenbank über die Patentanmeldungen der an den FIT-IT Projekten beteiligten Unternehmen zeigt eine sehr deutliche Zunahme der Patentierungsaktivität über die Laufzeit des FIT-IT-Programms, die mit einem deutlich steigenden Anteil der Bedeutung von Patenten in IKT-Technologiefeldern und der fortschreitenden Internationalisierung der Patentanmeldungen verbunden war.

Auch wenn nur ein geringer Anteil (10\%) der beteiligten Unternehmen in der Befragung über die Gründung von Spin-offs oder Start-ups als eine bleibende Nachwirkung der FIT-IT Förderung berichtete, dürfte damit das Programmziel, pro Förderjahr zumindest die Gründung eines neuen Unternehmens zu stimulieren, jedenfalls erreicht worden sein. In den sechs FITIT Projekten, die wir in den Fallstudien näher bezüglich ihrer weiteren Auswirkungen betrachtet haben, finden sich bereits zwei, die unmittelbar zu Firmengründungen geführt haben.

Die Angaben der Projektbeteiligten in der Befragung zur Pilotaktion ModSim sind durchwegs positiv. Mehrere ehemalige Partner aus dem universitären Bereich gaben an, die Förderaktion habe wesentlich zum Aufbau von Kompetenz und Arbeitsgruppen im Bereich der mathematischen Modellierung und Simulation beigetragen. Zahlreiche erstmals in den ModSim-Projekten verfolgte Ansätze wären in Folgeprojekten weiterentwickelt worden und würden noch heute Bedeutung in Forschung und Lehre haben. Auch einige der an ModSim-Projekten beteiligte Unternehmen gaben an, heute noch von den Ergebnissen zu profitieren, einerseits durch ein insgesamt besseres Verständnis der Möglichkeiten und Grenzen der verfolgten Lösungsansätze, andererseits durch die entwickelten Algorithmen und Verfahren selbst.

Demgegenüber konnten für die Konzeptinitiative "Forschung schafft Arbeit“ in der Evaluierung keine belastbaren Informationen über deren Nutzen und Nachhaltigkeit eruiert werden. In der FFG-Datenbank finden sich überhaupt nur fünf Einträge genehmigter Projekte, die den Förderkriterien der Konzeptinitiative entsprechen. Nur von einem dieser Projekte erhielten wir eine Rückmeldung in der Befragung, wonach die Durchführung des Vorhabens keine bleibenden Nachwirkungen im Unternehmen gehabt habe. 
Die Programmkonzeption und das Programmmanagement von FIT-IT wurden von den Programmbeteiligten durchwegs als exzellent beschrieben. Die Programmausrichtung, das Auswahlverfahren und die Programmdurchführung hätten Vorbildcharakter für andere nationale Förderprogramme.

Ein Aspekt der im Zusammenhang mit der ex-post Evaluierung des Impulsprogramms FIT-IT auch für die Weiterentwicklung der aktuellen Themeninitiative IKT der Zukunft im Auge behalten werden sollte, betrifft die doch beträchtliche Diskrepanz der Einschätzungen und Angaben der Förderempfänger in unseren Gesprächen und in der Befragung zu den Wirkungen der Förderung in den Unternehmen und den unabhängig davon aus anderen Quellen erhobenen Daten (d. h. FuE-Erhebung Statistik Austria, PREDICT-Datenbank der Europäischen Kommission). Die ausgesprochen positiven Einschätzungen der Programmwirkungen der BMVIT IKT-Förderung in unseren Gesprächen und in der Befragung spiegeln sich jedenfalls nicht in dieser Allgemeinheit im Vergleich geförderter und nicht-geförderter Unternehmen hinsichtlich der Entwicklung der internen FuE-Ausgaben und der FuE-Beschäftigten in der FuE-Statistik wider. Ein positiver Zusammenhang zwischen Programmförderung und Entwicklung dieser Kennzahlen konnte nur (eingeschränkt) für die Gruppe der KMU festgestellt werden.

Dabei ist natürlich zu beachten, dass auch die nicht FIT-IT geförderten Unternehmen, die in der FuE-Erhebung von Statistik Austria erfasst sind, zahlreiche andere öffentliche FuE-Förderungen nutzen konnten (und genutzt haben) und damit eine einfache Gegenüberstellung von Förderung und Förderzielen nicht unmittelbar möglich ist. Es bleiben jedoch trotzdem beträchtliche Zweifel an der Additionalität der öffentlichen Förderung bei Großunternehmen hinsichtlich der internen FuE-Ausgaben und der FuE-Beschäftigung. Möglicherweise sollten bei der Programmkonzeption und der Programmumsetzung andere Aspekte von Additionalität, wie Verhaltensadditionalität durch die Intensivierung der Zusammenarbeit von Forschungseinrichtungen und Unternehmen bzw. der erzielte Mehrwert durch eine anwendungsorientierte Ausbildung und Forschung an den Universitäten in den Vordergrund gestellt werden.

\subsection{Wie gut ist die Umsetzung von IKT der Zukunft bisher gelungen?}

Die hohe Qualität der Programmdurchführung wurde von den Förderempfängern auch in der Interims-Evaluierung des FIT-IT Nachfolgeprogramms IKT der Zukunft bestätigt. Die Programmteilnehmer sind mit der Abwicklung des Programms ausgesprochen zufrieden. Hervorgehoben wurden von den befragten Förderempfängern insbesondere die Informationen über das Programm sowie die Schnelligkeit der Fragenbeantwortung und der Rückmeldungen durch die FFG sowie die Kompetenz der FFG-Mitarbeiter/innen. Auch mit den Informationsveranstaltungen zu den Ausschreibungen sind die Förderempfänger überwiegend zufrieden.

\section{Was kann zur Qualität des Projektauswahlverfahrens gesagt werden?}

Auch wenn viele Programmteilnehmer mit abgelehnten Anträgen Kritik an den aus ihrer Sicht nicht immer nachvollziehbaren Förderentscheidungen, an nicht ausreichenden Rückmeldungen zu den Gründen der Ablehnungen und an den zu geringen Gesamtbudgets der Ausschreibungen übten, schätzen jene Personen, die auch Einblick in die Verfahren und Prozesse der Antragsbewertung und Antragsauswahl in der Themeninitiative IKT der Zukunft haben, die Qualität des Auswahlverfahrens - auch im Vergleich zu anderen Förderprogrammen sehr hoch ein. Die Evaluierung der Anträge durch die internationalen Fachgutachter/innen in englischer Sprache sei immer noch Good Practice. Die Zusammenarbeit zwischen BMVIT und 
FFG funktioniere gut: Die FFG schlägt die Gutachter/innen vor. Die Bestellung erfolgt durch das BMVIT. Jeder Projektantrag wird von drei internationalen Fachgutachter/innen online vorab bewertet. In der späteren Vor-Ort-Sitzung des Bewertungsgremiums arbeiten die Gutachter/innen in parallelen Panels. Ein Briefing durch die FFG vor den Panelsitzungen soll sicherstellen, dass über alle Panels hinweg die vorgegebenen Bewertungskriterien für die Anträge in gleicher Art und Weise angelegt werden. Die Fachgutachter/innen in den Panels reihen die von innen bewerteten Projekte vergleichend anhand eines Punkteschemas.

Eine große Herausforderung im Verfahren ist die Erstellung der finalen Reihung der bewerteten Anträge über die Panels hinweg, die von Vertretern der einzelnen Panels in einer gemeinsamen abschließenden Sitzung vorgenommen wird. In der Praxis tritt durchaus der Fall ein, dass einzelne Panels ihre Anträge generell strikter oder auch weniger strikt beurteilt haben als andere Panels. Hier kommt der gemeinsamen Diskussion und abschließenden Reihung der Projektanträge durch die Panelvorsitzenden noch eine wichtige Funktion zu.

Auch wenn eine systematische Verzerrung der Antragsbewertung durch die unterschiedlichen Gutachter/innen in den Panels nicht ausgeschlossen werden kann, stellt dieser Umstand die Qualität und Fairness des Verfahrens nicht grundsätzlich in Frage. Solange sichergestellt bleibt, dass im Sinne der Ausschreibungsziele ausschließlich qualitativ ausgezeichnet bewertete Anträge zur Förderung vorgeschlagen werden, spielt es bezüglich der Angemessenheit und Effektivität der Förderung keine Rolle, ob es im Falle einer anderen personellen Zusammenstellung der Panels eine etwas veränderte Reihung der Anträge über die gesamte Ausschreibung hinweg gekommen wäre.

Die FFG versucht darüber hinaus ohnehin eine möglichst hohe Qualität und Kontinuität im Begutachtungsverfahren dadurch zu erreichen, dass ein Teil der Fachgutachter/innen über mehrere Ausschreibungen hinweg zu den Panelsitzungen eingeladen wird. Im Pool der Fachgutachter/innen sollen sich außerdem die wissenschaftlich-fachliche Expertise und die Anwendungsexpertise die Waage halten. Als Zielgröße wird ein Anteil von 50 \% Fachgutachter/innen aus der Industrie angestrebt.

\section{Welche Wirkungen und welchen Nutzen sehen die Förderempfänger/innen?}

Bezüglich der bereits erreichten Ergebnisse und Wirkungen der Projektförderung in IKT der Zukunft zeigen die Ergebnisse der Befragung, dass rund zwei Drittel der Unternehmen ihre Entwicklungen zu Prototypen oder Pilotanwendungen weiterführen. Unter den beteiligten Unternehmen der mit Ende 2016 bereits abgeschlossenen IKT der Zukunft Projekte geben rund $40 \%$ an, eigene Produkte, Prozesse oder Dienstleistungen bereits am Markt zu haben, die Ergebnisse des Projekts nutzen. Ein etwa ebenso großer Anteil sieht einen längerfristigen positiven Effekt auf die Anzahl der FuE-Beschäftigten im Unternehmen. Die beteiligten wissenschaftlichen Einrichtungen der bereits abgeschlossenen IKT der Zukunft-Projekte haben zu einem Anteil von über $90 \%$ wissenschaftliche Ergebnisse der Projekte publiziert. In mehr als der Hälfte der abgeschlossenen Projekte konnten auch Master-Arbeiten fertiggestellt werden. Gleichzeitig berichten rund 20 \% der wissenschaftlichen Partner bereits beendeter Projekte über den Abschluss von Promotionen als ein Ergebnis des Projekts.

Insgesamt sehen die Projektpartner der IKT der Zukunft-Projekte den größten Nutzen der Teilnahme in der Stärkung ihrer Kooperationsnetzwerke und der FuE-Partnerschaften mit anderen Forschungseinrichtungen und Unternehmen in Österreich. Vor allem die wissenschaftlichen Einrichtungen berichten in diesem Zusammenhang über einen großen Nutzen zur Verbesserung des Verständnisses der Anforderungen und Bedürfnisse der Nutzer/innen. 
In den geförderten Konsortien der IKT der Zukunft-Projekte dominieren eindeutig die Forschungseinrichtungen: Festlegung des Arbeitsprogramms, Durchführung der Forschungsarbeit, Koordination mit den Partnern sowie Ergebnistransfer und Öffentlichkeitsarbeit werden eindeutig von den wissenschaftlichen Partnern bestimmt. Eine eher gleichrangige Rollenverteilung zwischen Wissenschaft und Unternehmen gibt es bei der Festlegung der Projektziele und der Anforderungen an die zu entwickelnden Lösungen. Trotz der dominierenden Rolle der Forschungseinrichtungen in der operativen Projektdurchführung gelingt es den Unternehmen trotzdem, Projektergebnisse wirtschaftlich zu verwerten. Fast zwei Drittel der beteiligten Unternehmen sehen sich in großem Umfang bei der wirtschaftlichen Verwertung der Projektergebnisse involviert. Hinweise auf Unternehmensgründungen aus IKT der ZukunftProjekten konnten wir im Rahmen der Interims-Evaluierung noch nicht finden.

\section{Ist das Programmdesign hinsichtlich IKT-Themenfelder, Anwendungsfelder und Quer- schnittsthemen angemessen?}

Während die IKT-Themenfelder von IKT der Zukunft bei den Programmteilnehmer/inne/n insgesamt auf hohe Akzeptanz stoßen, trifft die Änderung des inhaltlichen Programmdesigns im Vergleich zum Impulsprogramm FIT-IT mit der nunmehrigen Ausrichtung der Projekte auf spezifische Anwendungsfelder nicht auf ausschließliche Zustimmung. Durch die Dimension Anwendungsfelder würden stärker generisch ausgerichtete IKT FuE-Projekte nicht mehr eingereicht werden können. Außerdem wären die Alleinstellungsmerkmale des IKT Programms im Vergleich zu anderen thematischen Förderprogrammen des BMVIT geringer geworden. Zum Teil sei nicht mehr ausreichend klar, wie sich ein IKT der Zukunft-Projekt von einem IKTbezogenen Mobilität der Zukunft-, Energiesysteme der Zukunft oder Produktion der ZukunftProjekt unterscheidet bzw. unterscheiden sollte. Das IKT-Förderprogramm habe damit an eigenem Profil verloren, da anwendungsfeldorientierte IKT-Forschung auch in anderen thematischen Programmen adressiert werden würde. Innerhalb des angebotenen Förderportfolios würde es sowohl Programme brauchen, die Anwendungsfelder adressieren, aber auch solche, in denen wissenschaftlich ambitionierte generische Forschung und Entwicklung im Zentrum steht.

Einige der Programmteilnehmer gaben zudem an, dass es durch die inhaltliche Neuausrichtung der IKT-Programmförderung zu einer Verschiebung des Programmschwerpunkts in Richtung Software und Systeme gekommen sei, während stärker hardwareorientierte FuEThemen heute deutlich schwieriger unterzubringen seien als während der Laufzeit des Impulsprogramm FIT-IT.

Unter den Rückmeldungen der Förderempfänger/innen zur thematischen Ausrichtung des Programms finden sich daher viele mit dem Vorschlag, die Ausschreibungen von IKT der Zukunft sollten auch Anträge außerhalb der spezifisch angesprochenen Anwendungsfelder zulassen. Eine zu restriktive thematische Fokussierung der Ausschreibungen würde oft nicht die Möglichkeit bieten, auf die aktuellsten Entwicklungen in einem der adressierten IKT-Themenfelder einzugehen.

Während das Spannungsfeld IKT-Themen und Anwendungsfelder in den Interviews und in den Rückmeldungen der Befragung von den Programmteilnehmer/inne/n häufig thematisiert wurden, kamen die drei Querschnittsziele von IKT der Zukunft praktisch nicht zur Sprache. Lediglich der häufige Hinweis von Programmteilnehmer/inne/n, die thematisch-inhaltlichen Vorgaben an Projekte in den Ausschreibungen sollten ambitionierte, zukunftsweisende IKT FuE-Vorhaben nicht unmöglich machen, interpretieren wir als einen Hinweis, dass die dritte Dimension Querschnittsziele (neben den Themenfeldern und den Anwendungsfel- 
dern) als Einschränkung verstanden wird. Gleichzeitig gab es zahlreiche Vorschläge der Programmteilnehmer/inne/n, das BMVIT möge verstärkt themenübergreifende Projekte, bzw. Anträge zu Querschnittsthemen in den Ausschreibungen zulassen. Damit waren aber jedenfalls nicht die Querschnittsziele "Human-Centered Computing", "Schonender Umgang mit Ressourcen“, "Bewusster Umgang mit FuE-Daten“ und „Europäische Dimension“ gemeint, sondern generische Projekte an den Schnittstellen zwischen den IKT-Themen- und/oder Anwendungsfelder.

Unserer Einschätzung nach waren die drei Querschnittsziele des Programms in den bisherigen Ausschreibungen von IKT der Zukunft von ganz unterschiedlicher Bedeutung. Es käme auch einer Überforderung und künstlichen Überfrachtung des Programmdesigns gleich, sie in allen Themen und Förderschwerpunkten des Programms integrieren oder adressieren zu wollen. Wir halten die Querschnittsziele "Human-Centered Computing“, „Schonender Umgang mit Ressourcen", "Bewusster Umgang mit FuE-Daten" und "Europäische Dimension" daher eher als Orientierungspunkte bei der Vorbereitung und Gestaltung von spezifischen Ausschreibungen und Förderthemen geeignet, denn als Aspekte, die in jeder Ausschreibung bzw. bei jeden Förderthema unbedingt berücksichtigt bzw. von den Antragstellern aufgegriffen werden müssten.

\section{Ist die Programmplanung hinsichtlich der Ausschreibungsthemen angemessen?}

Das Thema Planungssicherheit bezüglich der Vorhersehbarkeit der Auswahl der Anwendungsfelder in den Ausschreibungen spielt für die Programmteilnehmer nur insofern eine Rolle, als dass ein thematisches Andocken an die ausgeschriebenen Themen möglich ist. Daher ist für die Programmteilnehmer das Vertrauen auf die regelmäßige jährliche Ausschreibung in den IKT-Programmen verbunden mit einem möglichst großen Förderbudget wichtiger, als das frühzeitige Wissen um die thematische Ausrichtung einer Ausschreibung, die ferne in der Zukunft liegt. Für viele Programmteilnehmer ist die wichtigste Ausschreibung ohnehin immer die nächste relevante Ausschreibung, unabhängig vom spezifischen Programm bzw. der thematischen Ausrichtung.

Die Hinweise der Förderempfänger zum Thema Planungssicherheit gehen daher wie bereits oben gezeigt hauptsächlich in die Richtung, keine zu einschränkende thematische Fokussierung bzw. Ausrichtung auf ein bestimmtes Anwendungsfeld in den Ausschreibungen vorzunehmen, sondern innerhalb der definierten Forschungsthemen der Förderinitiative IKT der Zukunft verstärkt auch wieder generische Forschungsthemen bzw. Projekte in offenen Anwendungsfeldern zuzulassen. Zu enge Förderthemen würden außerdem den Kreis der potenziellen Antragsteller limitieren und damit den Wettbewerb der besten Ideen einschränken.

Planungssicherheit bezüglich der Auswahl und Gestaltung der Themen ist unserer nach vor allem für den Programmeigentümer BMVIT und die FFG als Förderagentur wesentlich. Langfristige, mehrjährige Planungsprozesse bezüglich Inhalte und Budgets tragen jedenfalls dazu bei, den strategischen Fokus bei der Programmumsetzung von IKT der Zukunft besser im Auge zu behalten und sprunghafte Einzelmaßnahmen zu vermeiden. In diesem Sinne sollten die Planungsprozesse für die Ausschreibungen im BMVIT und der FFG jedenfalls möglichst langfristig angelegt werden.

\section{Hat sich das Instrument Leitprojekt bewährt?}

Die bereits vorhandenen Erfahrungen mit dem Instrument Leitprojekt zeigen, dass ein Leitprojekt deutlich mehr ist als ein großes kooperatives FuE-Projekt oder auch als ein Portfolio mehrerer kooperativer Projekte unter einer gemeinsamen Ausschreibungsklammer. Der 
erste wesentliche Unterschied zwischen Leitprojekt und kooperativen FuE-Projekt ergibt sich aus der Projektgenese: Während kooperative FuE-Projekte inhaltlich und organisatorisch bottom-up durch die Partner entwickelt werden, sind in Leitprojekten die top-down durch den Fördergeber vorgegebenen Anforderungen deutlich höher. Dies wird auch von den Projektbeteiligten so wahrgenommen.

Angesichts der Erfahrungen mit den bisher durchgeführten Ausschreibungen für Leitprojekte stellt sich auch die Frage wie ein ausreichender Wettbewerb in der Antragsphase sichergestellt werden kann. In der ersten Ausschreibung 2014 wurde nur ein Antrag für ein Leitprojekt eingereicht. In der zweiten Ausschreibung 2016 wurden drei Anträge auf Förderung gestellt. Mangelnder Wettbewerb im Ausschreibungsverfahren kann die Handlungs- und Gestaltungsmöglichkeiten des Fördergebers in der Projektauswahl einschränken. Sofern Antragsteller von einer hohen Erfolgswahrscheinlichkeit eines einzelnen Antrags aufgrund der spezifischen formalen, inhaltlichen oder institutionellen Kriterien ausgehen können, wird dies Einfluss auf Charakter und Inhalte des eingereichten Antrags haben. Im Antragsbewertungsund Auswahlverfahren sollten die Gutachter/innen von Leitprojekten besonders darauf hingewiesen werden, dass durchaus auch kein einziger Antrag für eine Förderung empfohlen werden kann, wenn die formale und inhaltliche Qualität der Anträge eine Förderempfehlung nicht rechtfertigen.

Die bereits vorliegenden Erfahrungen mit dem Leitprojekt Data Market Austria zeigen, dass Leitprojekte jedenfalls ein zweckmäßiges und wirkungsvolles Förderinstrument sein können, sofern das Thema und die angestrebten Ziele ausreichend spezifiziert und inhaltlich beschrieben werden können. Das größere Projektkonsortium führt zwar zu mehr organisatorischer Komplexität und erfordert mehr Koordination zwischen den Beteiligten. In der operativen Projektdurchführung werden die Arbeiten aber ohnehin in parallel laufenden Entwicklungssträngen - und damit in kleineren Subkonsortien - durchgeführt werden.

Um die Ergebnisse und Erfahrungen in den Leitprojekten wieder zusammenzuführen und für Dritte nutzbar zu machen, sollte der Fördergeber besonderes Augenmerk auf die Kommunikation im Konsortium, auf den Ergebnistransfer und auf die Öffentlichkeitsarbeit zur Diffusion der Ergebnisse über das Konsortium hinaus richten. Gerade hier lägen nach Einschätzung von Projektbeteiligten die spezifischen Stärken eines Leitprojekts gegenüber der klassischen kooperativen FuE-Projektförderung. Mit der begleitenden Ausschreibung von Sondierungsprojekten für das Inkubatoren-Programm im Leitprojekt Data Market Austria hat das BMVIT hier auch schon besondere Maßnahmen gesetzt.

\section{Ist der Umfang der Maßnahmen zu Öffentlichkeitsarbeit und Awareness-Bildung in der Themeninitiative IKT der Zukunft ausreichend?}

Insgesamt schätzen die Programmteilnehmer/innen die Informationsangebote über die Themeninitiative und über zukünftige Ausschreibungen sowie die Gestaltungs- und FeedbackMöglichkeiten bei Veranstaltungen des BMVIT bzw. der FFG als sehr gut ein. Die Personen, die bereits beim Start des Impulsprogramms FIT-IT als Programmteilnehmer oder im Programmmanagement involviert waren, gaben an, dass vor allem in den ersten Jahren des Impulsprogramms FIT-IT ein deutlich aktiverer Ansatz des Programmmanagements gewählt wurde, als dies heute der Fall ist. Das Angebot an proaktiven, programmbegleitenden Maßnahmen und Aktivitäten zur Mobilisierung und Kommunikation des Programms sei deutlich dichter und intensiver gewesen als heute: Es wurden Preise für die besten FIT-IT-Projekte des Jahres vergeben (unter anderem unter dem Motto "Höchstleistung bitte!") und Dialogveranstaltungen mit der österreichischen Forschungscommunity über die Weiterführung des 
Programms und neue Programmschwerpunkte veranstaltet. Auf Informationsveranstaltungen wurden Ergebnisse aktueller bzw. abgeschlossener Projekte breit vorgestellt und das Programmmanagement kümmerte sich sehr aktiv Kommunikation des Programms.

Diese Hinweise zu den Unterschieden zwischen FIT-IT und IKT der Zukunft müssen jedoch unter den besonderen Rahmenbedingungen des Impulsprogramms FIT-IT Mitte der 2000er Jahre gesehen werden. Einige der damaligen Programmansprüche wie etwa die Heranführung der österreichischen IKT-Forschung an das EU-Forschungsrahmenprogramm können als inzwischen erreicht gesehen werden. Gleichzeitig konnte das damalige inhaltliche Programmmanagement deutlich einfacher und agiler agieren als dies heute für die FFG der Fall ist. Einerseits gab es noch wenig Abstimmungsbedarf mit anderen nationalen Programmen gab und andererseits waren Förderinstrumente und Förderprozesse noch nicht in dem Ausmaß standardisiert und harmonisiert wie heute.

Aufgrund der enormen Bedeutung die IKT-bezogene Themen im Zusammenhang mit der Digitalisierung von Wirtschaft und Gesellschaft in der Forschungs- und Innovationsförderung in den letzten Jahren erhalten haben, sehen wir keinen besonderen Bedarf an AwarenessBildung. Vielmehr sehen wir die Herausforderung, die sich durch Digitalisierung ergebenden Chancen und Potenziale gerade über IKT FuE-Projekte durch die Vernetzung von Akteuren und den Transfer von Erfahrungen und Ergebnissen bestmöglich zu nutzen. In den Gesprächen mit Programmteilnehmer/inne/ $\mathrm{n}$ und in den Antworten der Befragungen wurde in diesem Zusammenhang mehrmals der Wunsch nach Begleitgruppen zu Clustern von thematisch verwandten Projekten bzw. mehr Aufmerksamkeit für den Transfer von Ergebnissen aus abgeschlossenen Projekten als gewünschte Maßnahmen genannt.

Ein Schritt in diese Richtung bietet bereits das Design der Leitprojekte in der Themeninitiative IKT der Zukunft, die ein klares Konzept für den Ergebnistransfer bzw. für die Einbeziehung Dritter in die Vorhaben enthalten müssen. Auf die vorliegenden Erfahrungen mit der Umsetzung dieser Konzepte sollte das BMVIT bzw. die FFG bei der Weiterentwicklung von Maßnahmen der Öffentlichkeitsarbeit und der Awareness-Bildung aufbauen. Die Vernetzung laufender Projekte untereinander und der Ergebnistransfer könnte durch verstärkte Begleitmaßnahmen ( $z$. B. Projektpräsentationen und regelmäßige Ergebnisaustausch während der Laufzeit der Projekte) unterstützt werden.

\subsection{Wie erfolgreich ist die österreichische Beteiligung an der Gemeinsamen Technologieinitiative ECSEL?}

Österreich hat sich sehr erfolgreich an den Gemeinsamen Technologieinitiativen beteiligt. Österreichische Projektpartner sind in 19 von 38, und damit in der Hälfte aller in den ECSELAusschreibungen der Jahre 2014 bis 2016 geförderten Projekte vertreten. Bezogen auf die Anzahl der Projektbeteiligungen liegt Österreich nach Deutschland (29), Niederlanden (27), Frankreich (26), Belgien (25), Italien (22) und Spanien (21) an siebter Stelle unter 26 Ländern, in denen Organisationen an ECSEL-Projekten beteiligt waren. Mit 29.5 Mio. Euro ECSEL EU-Beiträgen in den Jahren 2014 bis 2016 liegt Österreich sogar an sechster Stelle. Die wirtschaftlich doch deutlich größeren Länder Deutschland, Frankreich und Italien erhielten nur das 3,6-fache, 1,7-fache und 1,4-fache der den österreichischen Partnern zugesprochenen EU-Mittel. Nur die Niederlande (15,7\% ECSEL-Anteil versus 4,8\% BIP-Anteil unter den EU-Staaten) und Belgien (12,6 \% ECSEL-Anteil versus 2,8 \% BIP-Anteil) haben in den Jahren 2014 bis 2016 bezogen auf ihre Wirtschaftsleistung einen höheren ECSEL EU-Beitragsanteil als Österreich (6.7 \% ECSEL-Anteil versus 2,4 \% BIP-Anteil unter den EU-Staaten) erhalten. 
Die Hinweise aus den Interviews und der Befragung deuten für die beteiligten Forschungseinrichtungen während der Laufzeit des Impulsprogramms FIT-IT auf eine hohe Komplementarität der österreichischen Beteiligung an den Gemeinsamen Technologieinitiativen mit dem nationalen IKT-Förderprogramm FIT-IT hin. Zahlreiche an ARTEMIS, ENIAC und ECSEL beteiligte Forschungseinrichtungen gaben an, dass nationale (Vor-)Projekte im Impulsprogramm FIT-IT wesentlich gewesen seien, die inhaltlichen Vorarbeiten für die Einreichung von Anträgen in den Ausschreibungen der Gemeinsamen Technologieinitiativen zu leisten. Netzwerke und Kontakte, die bereits im Rahmen von ARTEMIS und ENIAC geknüpft wurden, seien auch in ECSEL-Projekten fortgeführt worden bzw. würden heute noch von Bedeutung sein.

Für die beteiligten Unternehmen hatte diese Türöffner-Funktion der nationalen FIT-IT-Förderung hingegeben weniger Bedeutung. Vor allem die laufend in Projekten integrierten groBen Unternehmen waren auch ohne nationale Vorprojekte in der Lage, Zugang zu geeigneten Kooperationen und Netzwerken zu finden. Die bereits früh eingerichteten nationalen Plattformen haben zudem zu einer effektiven Kommunikation und Vernetzung der österreichischen Schlüsselakteure untereinander und mit relevanten europäischen Partnern beigetragen.

Auffällig im Vergleich zu nationalen Projekten in den Programmen FIT-IT bzW. IKT der Zukunft ist auch die deutlich unterschiedliche Positionierung der Unternehmen innerhalb der Projektkonsortien. In ECSEL-Projekten sind es überwiegend die Unternehmen, und nicht die Forschungseinrichtungen, die Ziele, Anforderungen an Lösungen und das Arbeitsprogramm der Projekte festlegen. Sie sind stärker in die Koordination der Partner involviert und tragen auch selbst in größerem Umfang maßgeblich zur Durchführung der Forschungsarbeiten bei. Den beteiligten wissenschaftlichen Einrichtungen kommt bezüglich der strategischen Ausrichtung und Steuerung dieser Projekte nur eine untergeordnete Rolle zu.

Diese deutlich stärkere Rolle der Unternehmen spiegelt sich auch in der Einschätzung des Nutzens der Projektteilnahme und der Verwertung der Projektergebnisse wieder. Die langfristige Bedeutung der Projekte für die Mitarbeiter/innenqualifizierung und für den internen Know-how-Aufbau wird von den Unternehmen besonders hoch eingeschätzt. Auch die Nachwirkungen der Projekte bezüglich der Verbesserung der Zusammenarbeit mit Unternehmen werden deutlich größer eingeschätzt als das bei nationalen Projekten in den BMVIT IKT-Programmen der Fall ist. Außerdem werden die wirtschaftlichen Wirkungen der Projektteilnahme von den Unternehmen deutlich besser eingeschätzt als in nationalen Projekten. Vier von zehn Unternehmen (41\%), die an ARTEMIS und ENIAC-Projekten beteiligt waren, berichteten über Umsatzsteigerungen bzw. eine Verringerung der Kosten, während in nationalen FIT-IT Projekten dieser Anteil bei rund einem Viertel lag (24\%).

Ein ähnliches Bild ergibt sich aus der Befragung der jüngeren ECSEL-Projekte. Vier von fünf der beteiligten Unternehmen (84\%) gaben in der Befragung an, aufgrund der Projektteilnahme heute mehr FuE-Personal zu beschäftigen und fast zwei Drittel (63\%), dass bereits eigene Produkte, Prozesse oder Dienstleistungen auf dem Markt seien, die Ergebnisse des Projekts nutzen. Die entsprechenden Anteile bei nationalen IKT der Zukunft-Projekten liegen bei $36 \%$ (mehr FuE-Personal) bzw. 28\% (Produkte. Prozesse oder Dienstleistungen am Markt). ECSEL-Projekte dürften damit in den Unternehmen sehr deutlich marktnäher und damit auch wettbewerblich ausgerichtet sein als IKT der Zukunft-Projekte.

Bei den beteiligten Forschungseinrichtungen zeigt sich hingegen ein anderes Bild. Interessanterweise ziehen die beteiligten Forschungseinrichtungen aus der Teilnahme an ECSELVorhaben vor allem auch wissenschaftlichen Nutzen. So können die wissenschaftlichen Partner in ECSEL-Projekten deutlich häufiger bzw. rascher Promotionen abschließen (25\%) als in 
nationalen IKT der Zukunft-Projekten (12\%). Sowohl die Möglichkeiten der Publikation von wissenschaftlichen Ergebnissen als auch der Beitrag zur Verbesserung der Ausbildung von Nachwuchswissenschaftler/inne/ $\mathrm{n}$ wird in ECSEL-Projekten deutlich höher bewertet als in nationalen IKT der Zukunft-Vorhaben.

Gleichzeitig wurde aus den Hinweisen der Unternehmen und Forschungseinrichtungen zu den Projekten der Gemeinsamen Technologieinitiativen deutlich, dass der additionale Effekt der Projektförderung bei den Unternehmen nicht überschätzt werden sollte. Vertreter/innen von Forschungseinrichtungen gaben an, Unternehmen in ECSEL-Projekten würden häufig eigenen Entwicklungsschwerpunkten folgen und nur an wenigen Partnern tatsächlich inhaltliches Interesse haben. Gleichzeitig sind die Projekte der Gemeinsamen Technologieinitiativen für die Unternehmen häufig von hoher strategischer Bedeutung.

Dies führte in der Befragung der Programmteilnehmer/innen zu einigen vordergründig paradoxen Ergebnissen. In keinem anderen Förderthema wurde und wird das Ausmaß der Förderung (das heißt die Förderhöhe) so kritisch beurteilt, wie in den Projekten der Gemeinsamen Technologieinitiativen. Nur 15 \% der Unternehmen in ECSEL-Projekten sind mit der Förderhöhe zufrieden oder sehr zufrieden, während in IKT der Zukunft-Projekten dies 59 \% der Unternehmen sind. Gleichzeitig hat aber über die Jahre der Förderanteil unter den drei Förderthemen und das absolut bewilligte Fördervolumen für Projekte der Gemeinsamen Technologieinitiativen zugenommen. Auch hat die Absenkung der durchschnittlichen Förderquote für die Unternehmen in Projekten der Gemeinsamen Technologieinitiativen - wie auch in der Zwischenevaluierung bereits angeregt - von etwa 40 \% in ARTEMIS und ENIAC während der Laufzeit von FIT-IT auf nunmehr rund $30 \%$ in ECSEL seit Start der Themeninitiative IKT der Zukunft keineswegs zu einer geringeren Beteiligung der Unternehmen geführt, sondern vielmehr zu einer deutlichen Ausweitung der Projektvolumina (von durchschnittlich 1,3 Mio. Euro Gesamtkosten auf 2,3 Mio. Euro Gesamtkosten).

Aus diesen Ergebnissen ziehen wir den Schluss, dass der Mehrwert der ECSEL-Projekte für die Unternehmen vor allem in der europäischen Konzertierung liegt und nicht unbedingt in der Finanzierung der Forschung. Radikal zu Ende gedacht läge daher die ausschließliche öffentliche Finanzierung dieser Konzertierungskosten nahe, ähnlich dem Model der Finanzierung von COST Actions. Aus förderökonomischer Sicht erscheint jedenfalls die Reduzierung der Förderquote für Unternehmen noch nicht ausgereizt zu sein. Für die beteiligten Forschungseinrichtungen - vor allem für die Hochschulen - sollten hingegen aufgrund des klar wissenschaftlichen Charakters ihrer Projektbeiträge und aufgrund der Bedeutung für die Ausbildung von Doktorand/inn/en für den wissenschaftlichen Nachwuchs und für die Wirtschaft bessere Modelle der (Aus-)Finanzierung angedacht werden.

\subsection{Welche Erfahrungen liegen für die Umsetzung des Förderschwer- punkts Demographischer Wandel vor?}

Die Projekte zum Demographischen Wandel - sowohl auf nationaler als auch auf transnationaler Ebene - sind nach wie vor unter den drei Förderthemen der Themeninitiative IKT der Zukunft die strukturell und organisatorisch ambitioniertesten. Durch die Einbeziehung von nicht-klassischen FuE-Akteuren und dem Fokus auf Lösungen für (End-)Anwender/innen unterscheiden sich diese Projekte sehr deutlich von den Vorhaben der sonstigen IKT-FuESchwerpunkte der Themeninitiative. Projekte zum Demographischen Wandel sind im Vergleich der drei Förderschwerpunkte jene, in denen bei der Projektdurchführung von den Beteiligten ein besonders breiter Mix an Herausforderungen wahrgenommen wird. Diese betreffen nicht nur technische oder organisatorische Aspekte, sondern stehen auch mit den 
verfügbaren Kompetenzen und Ressourcen der Partner in Zusammenhang. Trotz - oder vielleicht auch gerade wegen - dieser besonderen Herausforderungen schätzen die beteiligten Unternehmen die Intensität der Zusammenarbeit mit den Anwender/inne/n und mit anderen Unternehmen als besonders hoch ein. Es gelingt den Projekten auch vergleichsweise gut, eines der Hauptziele der Förderung zu erreichen, nämlich bei den Unternehmen das Verständnis über die Anforderungen und Bedürfnisse der Nutzer/innen und Endanwender/innen zu erhöhen.

Die Aufrechterhaltung der Kontakte mit den Partnern des Projekts nach Projektende fält im Förderschwerpunkt Demographischer Wandel jedoch schwerer als in Projekten der anderen Förderschwerpunkte. Die beteiligten Partner wiesen in diesem Zusammenhang beispielsweise auf mangelnde Möglichkeiten der (öffentlichen) Folgefinanzierung von geförderten Projekten hin, gerade auch an der Schnittstelle zwischen nationalen benefit-Projekten und transnationalen AAL-Projekten.

Die geförderten transnationalen Projekte des Förderschwerpunkts Demographischer Wandel sind bezüglich der Gesamtkosten je Partner nach wie vor signifikant größer als jene im nationalen Programm benefit. Wie bereits in der Evaluierung der österreichischen Beteiligung am AAL Joint Programme festgestellt, erlaubt die nationale Programmschiene die Erprobung neuer Förderansätze, was insbesondere auch durch die Förderung der Modellregionen zum Ausdruck kommt.

Der Anteil der Unternehmen in Projekten zum Demographischen Wandel, die über bereits am Markt befindliche Produkte, Prozesse oder Dienstleistungen berichten, die auf Ergebnissen der Projekte aufbauen, ist im Vergleich zu Befragung im Jahr 2015 in etwa gleichgeblieben. In der aktuellen Befragung gaben 30 \% der Unternehmenspartner an, eigene Produkte, Prozesse oder Dienstleistungen am Markt zu haben, die Ergebnisse des Projekts nutzen und weitere 26 \% berichteten über Produkte, Prozesse oder Dienstleistungen von Projektpartnern.

Auch wenn die Überschneidung der Zielgruppen im Unternehmensbereich zwischen dem Förderschwerpunkt Demographischer Wandel und den anderen Förderschwerpunkten sehr gering ist - weniger als $4 \%$ der geförderten Partner aus Wirtschaft und Praxis wurden bereits auch in einem der beiden anderen Förderschwerpunkte gefördert (der Vergleichswert im Förderschwerpunkt IKT der Zukunft beträgt 10 \% bzw. im Förderschwerpunkt ECSEL 31 \%) spricht grundsätzlich nichts gegen die Positionierung des Förderschwerpunkts in der Themeninitiative IKT der Zukunft. Durch die inhaltliche Gestaltung und Ausrichtung der Ausschreibungen sowie durch die Ansprache und Betreuung sehr heterogener Zielgruppen, ist in den vergangenen zehn Jahre im Förderschwerpunkt Demographischer Wandel spezielle Expertise aufgebaut worden, die in Zukunft auch in anderen komplexen, primär nicht technisch orientierten IKT-Anwendungsfeldern von Nutzen sein könnte. 


\section{$7 \quad$ Empfehlungen zur Fortführung von IKT der Zukunft}

Die Umsetzung der Themeninitiative IKT der Zukunft sollte in Zukunft auf in dieser Evaluierung sehrdeutlich gewordenen Stärken des Programms, vor allem hinsichtlich der Steuerung, Organisation und Umsetzung sowie der breiten Beteiligung und Kommunikation mit den Zielgruppen der Förderung weiter aufbauen. Handlungsfelder für die Anpassungen bei der Umsetzung der Themeninitiative sehen wir neben in den vorherigen Abschnitten bereits erwähnten Punkten vor hinsichtlich der Positionierung des nationalen Förderschwerpunkts IKT der Zukunft innerhalb des Programmportfolios des BMVIT, der damit zusammenhängenden Gestaltung der Förderschwerpunkte und der Förderinstrumente sowie beim Programmmonitoring.

\subsection{Förderschwerpunkt IKT der Zukunft auf wissenschaftlich ambitionierte, generische FuE-Projekte ausrichten}

Durch die Verflechtung der Förderthemen in IKT der Zukunft an Anwendungsfeldern haben sich zahlreichen Schnittflächen zu anderen thematischen Förderprogrammen des BMVIT ergeben, in denen ebenfalls IKT-bezogene Forschungsfragen adressiert werden können (z. B. Produktion der Zukunft, KIRAS, Mobilität der Zukunft, Stadt der Zukunft, Elektromobilität, Smart Grids). Um die Abgrenzung des Förderschwerpunkts zu stärken, könnte IKT der Zukunft stärker auf wissenschaftlich besonders ambitionierte Forschungsvorhaben zwischen Forschungseinrichtungen und Unternehmen ausgerichtet werden. Dies könnte weiterhin entlang der bestehenden Themenfelder und Querschnittsthemen des Programms erfolgen und auch primär Vorhaben unterstützen, die einen Beitrag zur Lösung von Herausforderungen in einem oder mehreren Anwendungsfeldern erwarten lassen, ohne diese jedoch unmittelbar ins Zentrum zu stellen.

Unser Vorschlag würde darauf abzielen, in IKT der Zukunft bevorzugt besonders ambitionierte Projekte der industriellen Forschung zu fördern, die auch einen entsprechend hohen Beitrag an wissenschaftlicher Forschung vor allem aus dem Bereich der Hochschulen erfordern. Projekte der experimentellen Entwicklung sowie Vorhaben, die überwiegend durch die internen Forschungsleistungen der Unternehmen getragen werden, könnten hingegen bevorzugt in anderen thematischen Programmen des BMVIT (oder anderer Programmeigentümer) gefördert werden. Durch eine stärkere Orientierung auf wissenschaftliche Grundlagen und besonders ambitionierte Forschungsfragen könnten die Programmziele "Spitzentechnologien weiterentwickeln" und "Spitzenkräfte bereitstellen und gewinnen" besonders unterstützt werden. Ein solcher Fokus von IKT der Zukunft auf wissenschaftlich besonders ambitionierte Forschung ließe sich auch sehr gut mit strukturell wirksamen Maßnahmen wie Stiftungsprofessuren kombinieren. Unser Vorschlag bedeutet aber nicht, bloß wissenschaftsgetriebene Vorhaben zu fördern. Im Gegenteil: Die Konsortien der Projekte sollten durchaus strukturell anspruchsvoll sein, etwa in dem mehrere Forschungspartner und Unternehmen gemeinsam an komplexeren FuE-Fragen zusammenarbeiten.

\subsection{Durch Instrumentenmix die Strukturwirkung von Förderung stärken}

Auf die Frage des BMVIT in diesem Evaluationsauftrag, ob es sinnvoller erscheint, mehrere Leitprojekte (FFG-Instrument Leitprojekt) auszuschreiben oder eine größere Anzahl von kleineren Projekten (FFG-Instrument kooperatives FuE-Projekt bzw. Sondierungsprojekt) zu fördern können wir zusammenfassend antworten, dass je nach Förderziel und Förderbedarf 
weiterhin ein Mix an Instrumenten eingesetzt werden sollte. Sofern Leitprojektthemen ausreichend inhaltlich spezifiziert werden können, spricht nichts gegen eine Ausweitung der Nutzung des Instruments in der Themeninitiative IKT der Zukunft. Leitprojekte bieten den Vorteil, dass damit die national vorhandenen Akteure und deren Ressourcen zu einem bestimmten Thema unmittelbarer zusammengeführt und koordiniert werden können als dies mit einem Bündel von kooperativen FuE-Projekten möglich wäre. Aufgrund der vergleichbar marktnahen Anforderungsbeschreibung des Konzepts Leitprojekt, wäre zu überlegen, ob das Instrument bei den beteiligten Unternehmen vor allem die Förderung experimenteller Entwicklung im Fokus haben sollte.

Gleichzeitig erachten wir es jedoch als sinnvoll, auch weiterhin ambitionierte kooperative FuE-Projekte im Programm IKT der Zukunft zu fördern, die bottom-up durch Forschungseinrichtungen und Unternehmen ausgearbeitet werden, vor allem wenn die generische Ausrichtung der Themeninitiative im Vergleich zu stärken anwendungsfeldorientierten Programme des BMVIT in Zukunft gestärkt werden sollte. Sondierungsprojekte könnten zur Unterstützung spezifischer Programmziele oder auch als Begleitmaßnahmen für laufende Leitprojekten eingesetzt werden, wie sie mit der Ausschreibung 2018 bereits auch erfolgt ist.

Die inhaltlichen Ansprüche und die Größe kooperativer FuE-Projekte in der Themeninitiative IKT der Zukunft sollte jedenfalls die Beschäftigung von Doktorand/inn/en möglich machen, wobei ein entsprechendes Konzept bereits im Antrag gefordert werden könnte. Auch in dieser Evaluierung wurde von vielen Gesprächspartner/inne/n (zum wiederholen Male) auf die nach wie vor zentrale Bedeutung der Stärkung der universitären Ausbildung von IKT-Spezialisten und Nachwuchswissenschaftler/innen hingewiesen. Auch wenn es nicht in die primäre Zuständigkeit des BMVIT fällt, für eine zukunftsgerichtete Ausbildungs- und Forschungsinfrastruktur an den österreichischen Hochschulen zu sorgen, sollte das BMVIT weiterhin die Einrichtung von programmstrategisch als kritisch erkannte Stiftungsprofessuren fördern.

Ausschließlich durch Unternehmen getragene Projekte, bzw. Vorhaben von Unternehmen, in denen öffentliche Forschungseinrichtungen nur in untergeordneter Rolle (z. B. im Unterauftrag) vertreten sind, sollten an das FFG-Basisprogramm verwiesen werden. Sollten im Einzelfall derartige Projekte doch gefördert werden, sollten die Projekte die gleichen Fördersätze erhalten, wie bei einer Förderung im FFG-Basisprogramm (bzw. bei internationalen Vorhaben als EUREKA-Projekt) zu erwarten gewesen wäre.

\subsection{FFG Erhebungen für das Monitoring des Programms nutzen}

Eine der Fragen in der Leistungsbeschreibung in dieser Evaluierung war, wie aussagekräftig die gewählten Indikatoren der Themeninitiative IKT der Zukunft sind, um die Zielerreichung des Programms zu messen und ob es gegebenenfalls im Hinblick auf die Programmziele besser geeignete Indikatoren gäbe.

Grundsätzlich sollten die Indikatoren so gewählt werden, dass sie zum einen die Programmziele möglichst gut abbilden und zum anderen durch das BMVIT bzw. die FFG auch erhoben bzw. nachverfolgt werden können. Für die Steuerung und kontinuierliche Weiterentwicklung des Programms ist es zudem wünschenswert, sich auf Indikatoren zu konzentrieren, die mit möglichst einfachen Mitteln, vergleichbar, regelmäßig bzw. zeitnahe erhoben werden können. Eine Herausforderung im Kontext des Programms stellen auch die Spezifika der drei Förderschwerpunkte dar, die aufgrund ihrer Ausrichtung und Zielsetzung jeweils besondere 
Indikatoren zweckmäßig erscheinen lassen. Positiv an den aktuell festgelegten Zielindikatoren für die Themeninitiative IKT der Zukunft ist anzumerken, dass ein Teil unmittelbar durch die Auswertung der FFG-Antrags- und Förderdaten erhoben bzw. überprüft werden kann.

Allerdings würden wir mittelfristig für das Monitoring der Themeninitiative IKT der Zukunft vorschlagen, als Informationsgrundlage zusätzlich verstärkt die durch die FFG beauftragte Befragung der Förderempfänger der jeweils vier Jahre zuvor abgeschlossenen Projekte über die erzielten Effekte und Wirkungen der Förderung zu nutzen. Diese Befragung wird jährlich für alle FFG-geförderten Projekte durchgeführt. Durch eine über die Erhebungsjahre hinweg kumulierte Auswertung der Ergebnisse der Wirkungsbefragung könnten beispielsweise Informationen zum time-to-market, zu den gegebenenfalls erzielten Umsätzen und zur Beschäftigungswirkung durch Verwertung der Projektergebnisse für die Laufzeit der Themeninitiative hinweg erhoben werden.

Ein wesentlicher Vorteil der Nutzung der Erhebungsdaten der FFG aus der Wirkungsbefragung gegenüber Befragungen im Rahmen von einmaligen ex-post Befragungen im Zuge von Programmevaluationen ist, dass alle abgeschlossenen Projekte immer zum gleichen Zeitpunkt nach Projektende befragt werden. Außerdem ist beim Nutzen der Daten der FFG-Wirkungsbefragung ein Vergleich der Förderwirkungen mit anderen Programmen und Themeninitiativen unmittelbar möglich. Dies würde die Abstimmung bzw. Abgrenzung der unterschiedlichen Förderinstrumente und Förderprogramme des BMVIT unterstützen. Wir schlagen daher vor, dass das BMVIT mit der FFG gemeinsam eine Vorgehensweise erarbeitet, die zukünftig die Nutzung der Daten aus der FFG-Wirkungserhebung programmbezogen und über den Zeitverlauf der Programme hinweg möglich macht. Bezüglich der aktuellen (und möglicher zukünftiger) Indikatoren und Kennzahlen zu den strategischen Zielen der Themeninitiative IKT der Zukunft möchten wir im Lichte der Evaluierung die folgenden Hinweise geben:

Zum strategischen Ziel „Steigerung der Quantität und Qualität der IKT-Forschung und -Entwicklung, die dazu geeignet ist, Technologieführerschaft zu erringen und beizubehalten" strebt das BMVIT an, über die Programmlaufzeit im Mittel mehr als neue $10 \%$ der Teilnehmer/innen im Programm bzw. mehr als $5 \%$ erstmals FFG geförderte Einrichtungen zu erreichen. Die Beteiligungszahlen seit 2012 zeigen, dass es der Themeninitiative IKT der Zukunft jedenfalls gelingt diese Zielwerte zu erreichen bzw. zum Teil deutlich zu übertreffen. Als einen weiteren möglichen Indikator, der aus dem FFG-Wirkungsmonitoring übernommen werden könnte, böte sich der Anteil der Unternehmen an, die vier Jahre nach Projektende über eine Verbesserung ihrer technologischen Wettbewerbsposition durch die Ergebnisse des FuE-Projekts berichten. Laut aktuellem FFG-Wirkungsmonitoringsbericht lag der Durchschnittswert für Unternehmen in kooperativen FuE-Projekten, die über eine positive Veränderung ihrer technologischen Wettbewerbsposition berichteten (Position verbessert, zur Spitze aufgeschlossen oder Spitze gehalten) bei $68 \%$ (nationale Wettbewerbsposition) bzw. $61 \%$ (internationale Wettbewerbsposition).

Der genannte Indikator würde unserer Ansicht nach auch das strategische Ziel „Stärkung der Innovationsleistung österreichischer IKT-affiner Unternehmen im globalen Wettbewerb der Innovationen" abbilden helfen. Derzeit wird dieses Ziel durch den Indikator Erhöhung des KMU-Anteils in den Projektkonsortien operationalisiert, wobei als Kennzahl ein Wert von gröBer als $25 \%$ KMU-Anteil festgelegt ist. Möglicherweise wäre es hier aussagekräftiger, den KMU-Anteil auf die insgesamt beteiligten Unternehmen zu beziehen und zwischen den drei Förderthemen stärker zu differenzieren. Der KMU-Anteil unter den geförderten Unternehmen variierte bisher von 16 \% in Projekten der Gemeinsamen Technologieinitiativen bis $86 \%$ in den geförderten Projekten zum Demographischen Wandel. Ein weiterer Indikator, der aus 
dem FFG-Wirkungsmonitoring zusätzlich übernommen werden könnte, ist der Anteil der Unternehmen, der Ergebnisse der Projekte vier Jahre nach Projektende wirtschaftlich verwertet. Laut aktuellem FFG-Wirkungsmonitoringsbericht lag der Durchschnittswert für Unternehmen in kooperativen FuE-Projekten, die Ergebnisse wirtschaftlich verwerten bei $32 \%$, wobei weitere 33 \% über einen Erkenntnisgewinn berichteten.

Zum strategischen Ziel „Positionierung österreichischer F\&E-Akteure in IKT-Forschungsfeldern mit hohem Potenzial" sieht das BMVIT den Indikator Peer-reviewed Publikationen mit einem Zielwert von mindestens drei pro Projekt bei Projektende vor. Eine Erhebung des Indikators wäre aktuell nur durch eine Auswertung der Projektendberichte möglich und mit substanziellem Aufwand für die FFG verbunden. Für das Vorgängerprogramm FIT-IT konnten wir in der Evaluation mit den bibliometrischen Auswertungen zeigen, dass der vorgesehene Zielwert mit hoher Wahrscheinlichkeit deutlich übertroffen wurde. Aus dem FFG-Wirkungsmonitoring könnten zusätzlich die Indikatoren „Anteil der Forschungseinrichtungen mit Projektpublikationen in referierten Journals / Konferenzen“ und „Anteil der Forschungseinrichtungen, die das Projekt als Referenzprojekt für Auftragsprojekte nutzen konnten" für das Monitoring des strategischen Ziels "Positionierung österreichischer F\&E-Akteure in IKT-Forschungsfeldern mit hohem Potenzial" verwendet werden.

Andere Zielindikatoren sind unserer Ansicht nach hingegen nicht bzw. nicht zweckmäßig für alle Förderschwerpunkte der Themeninitiative IKT der Zukunft zu erheben. So ist nicht klar, warum das Ziel „Erhöhung der Lebensqualität durch neue IKT-Anwendungen" auch für Projekte der Gemeinsamen Technologieinitiative bzw. für die Ausschreibungen im Förderschwerpunkt IKT der Zukunft angewendet werden sollten. Gleiches gilt für den Indikator „Einbindung der Bedarfsträger in die Projekte". Auch die Definition bzw. Klassifikation von "gesellschaftlichen Herausforderungen" im Kontext der Ausschreibungen der Förderschwerpunkte Gemeinsamen Technologieinitiative und IKT der Zukunft bleibt offen.

Hinsichtlich des strategischen Ziels "Unterstützung österreichischer IKT-affiner Unternehmen bei Gründung und Wachstum" stellt sich die Frage, ob die Ausrichtung und die Schwerpunkte des Programms alleine mit dem Indikator "Anzahl von Spinn offs, Start ups“ angemessen abgebildet werden. Selbstverständlich kann die Gründung neuer Unternehmen ein wesentlicher Effekt der BMVIT IKT-Förderung sein (wie auch die Befragung der FIT-IT-Programmteilnehmer/innen zu den langfristigen Wirkungen der Förderung und die Fallstudien belegen). Möglicherweise wäre hier aber eine Fokussierung des Ziels bzw. der Monitoringaufgaben auf bestimmte Förderthemen bzw. Initiativen zweckmäßig. Beispielsweise stellt die Ausschreibung 2018 von Sondierungsprojekten für den Datenmarkt unmittelbar auf die Unterstützung des Inkubatorenprogramms von Data Market Austria ab (und damit auch auf Gründungen und Spin-offs) während in den Projekten der Gemeinsamen Technologieinitiative eher der Aspekt "Wachstum" (auch von international führenden großen Unternehmen) als Zielkategorie im Vordergrund steht. Als wachstumsgezogenen Indikator könnten aus dem FFG-Wirkungsmonitoring die Beschäftigungseffekte (d. h. geschaffene Arbeitsplätze und Rekrutierung von FuE-Personal) durch die wirtschaftliche Verwertung der Projektergebnisse ergänzend herangezogen werden. 


\section{Anhang 1 Kennzahlen zur Entwicklung des IKT-Sektors in Österreich}

Die Gemeinsame Forschungsstelle (JRC) der Europäischen Kommission führt seit 2006 jährlich eine Untersuchung mit dem Titel "Prospective Insights in ICT R\&D“ (PREDICT) durch, für die ein laufend aktualisierter Datensatz mit Monitoring-Indikatoren für die Mitgliedsländer der EU und internationale Wettbewerber erstellt und fortgeschrieben wird. Dieses Datensatz dient dazu, die Wirkungen der Lissabon Agenda und der EU2020 Strategie, der Digitalisierungsagenda für Europa sowie die Maßnahmen der Mitgliedsländer im Bereich der IKT-Forschung bzw. der Verwirklichung des Gemeinsamen Digitalen Marktes verfolgen zu können. Der Datensatz enthält auf Ebene der Mitgliedsländer international vergleichbare Daten zum Beitrag des IKT-Sektors zum Bruttoinlandsprodukt, zu den staatlichen Ausgaben für FuE im IKT-Sektor, zu den Unternehmensausgaben für FuE sowie deren Finanzierung sowie zu den FuE-Beschäftigten. Datengrundlage für den PREDICT-Datensatz bilden jeweils die letzten verfügbaren amtlichen Daten des Statistischen Amtes der Europäischen Union (Eurostat) und der Organisation für wirtschaftliche Zusammenarbeit und Entwicklung (OECD). Der Datensatz ist öffentlich zugänglich und wurde für diese Evaluierung dazu genutzt, die Entwicklung des österreichischen IKT-Sektors seit 2002 nachzuzeichnen.

Bei der Interpretation der Daten im Zusammenhang mit den Fragestellungen in dieser Evaluation ist zu beachten, dass die Zielgruppe des Impulsprogramm FIT-IT bzW. nunmehr jene der Themeninitiative IKT der Zukunft nicht nur den IKT-Sektor im engeren Sinne umfasst, sondern nicht zuletzt aufgrund der Digitalisierung eine zunehmend breitere Schnittmenge unterschiedlicher Wirtschaftsbereiche adressiert. Trotzdem bilden nach wie vor Unternehmen des IKT-Sektors im produzierenden und im Dienstleistungssektor den Kern bzW. sind Multiplikatoren für IKT-induzierte Veränderungen in der breiteren Wirtschaft. Von den Antragstellern im Impulsprogramm FIT-IT sind nur rund $20 \%$ der einreichenden produzierenden Unternehmen dem IKT-Sektor im engeren Sinn zuzurechnen, jedoch rund $60 \%$ der einreichenden Dienstleistungsunternehmen.

In den Auswertungen umfasst der produzierende Bereich des IKT-Sektors die Wirtschaftsklassen 26.1-26.4 (Herstellung von elektronischen Bauelementen und Leiterplatten, Herstellung von Datenverarbeitungsgeräten und peripheren Geräten, Herstellung von Geräten und Einrichtungen der Telekommunikationstechnik, Herstellung von Geräten der Unterhaltungselektronik) sowie 26.8 (Herstellung von magnetischen und optischen Datenträgern). Im Dienstleistungsbereich des IKT-Sektors sind die Wirtschaftsklassen 58.2 (Verlegen von Software), 61 (Telekommunikation), 62 (Erbringung von Dienstleistungen der Informationstechnologie), 63.1 (Datenverarbeitung, Hosting und damit verbundene Tätigkeiten; Webportale) und 95.1 (Reparatur von Datenverarbeitungs- und Telekommunikationsgeräten) zusammengefasst.

Die Auswertung der PREDICT-Daten zeigt, dass die staatlichen FuE-Mittel (Government budget appropriations or outlays for research and development - GBOARD) für IKT von 121 Mio. Euro im Jahr 2006 auf 215 Mio. Euro im Jahr 2015 zugenommen haben. Dies entspricht einer durchschnittlichen jährlichen Steigerung der staatlichen FuE-Mittel für IKT von 6,6 \%. 
Abbildung 45 Staatliche FuE-Mittel (GBOARD) Österreichs für IKT 2006 bis 2015 (Mio. Euro)

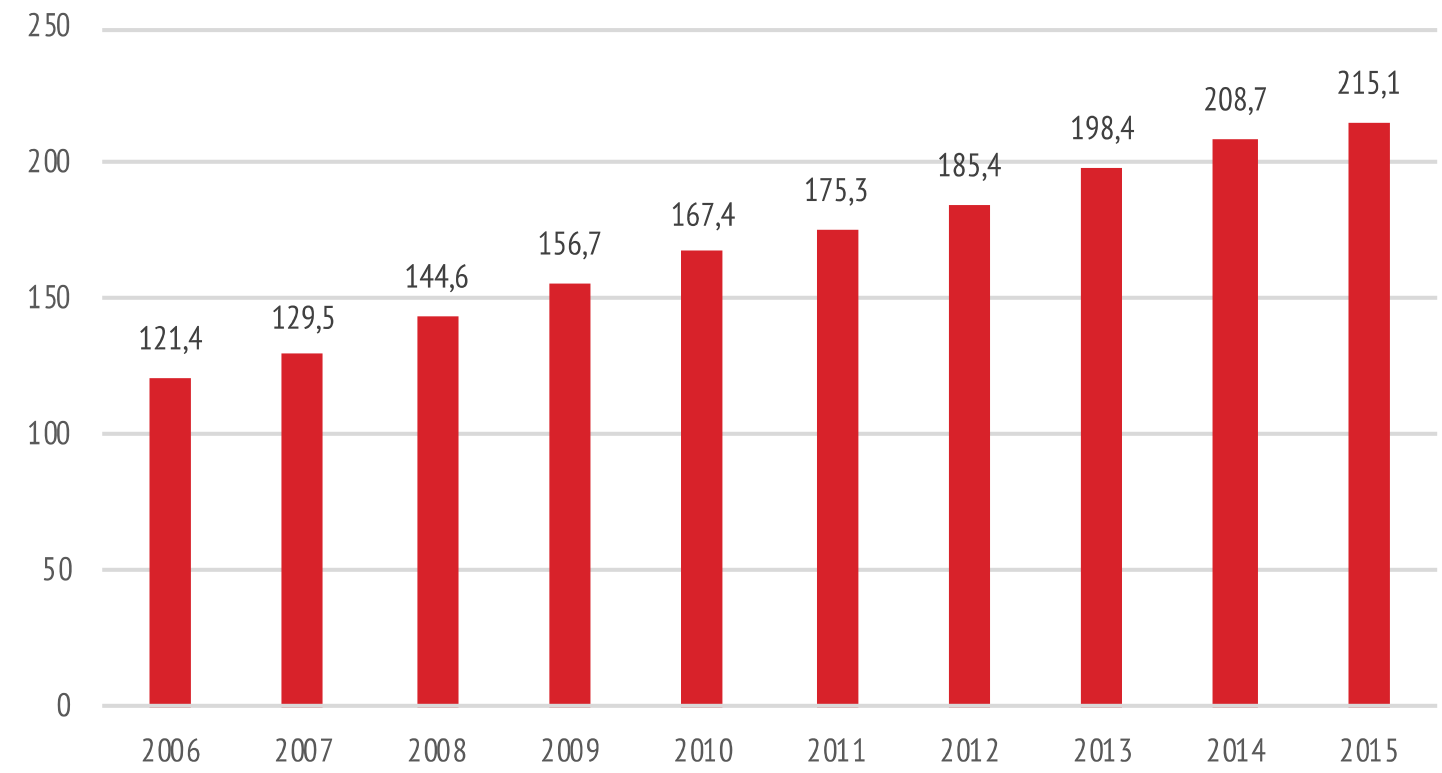

Quelle: EC PREDICT-Datenbank 2017; Berechnung und Darstellung inspire research

Besonders deutlich bezog sich die Zunahme der staatlichen FuE-Mittel für IKT in diesem Zeitraum auf die Mittel, die der industriellen Forschung und Produktion zur Verfügung gestellt wurden. Die staatlichen FuE-Mittel für dieses Forschungsziel stiegen zwischen 2006 und 2015 um 182 \% an, während beispielsweise die IKT-Grundlagenforschung über die allgemeine Hochschulfinanzierung nur um $51 \%$, die Grundlagenforschung für IKT ohne Berücksichtigung der allgemeinen Hochschulfinanzierung um $71 \%$ zunahm.

Abbildung 46 Staatliche FuE-Mittel (GBOARD) Österreichs für IKT 2006 bis 2015 nach Forschungszielen (Mio. Euro)

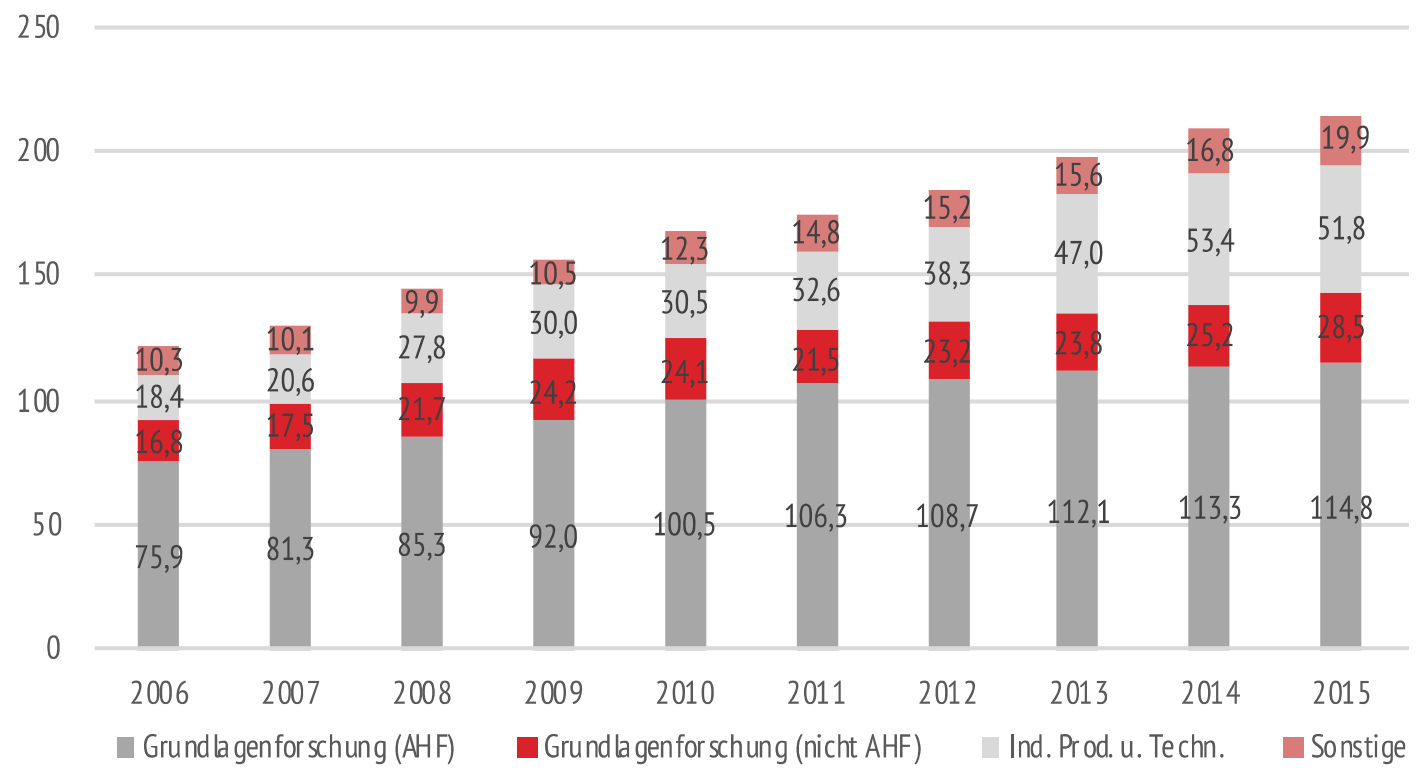

Quelle: EC PREDICT-Datenbank 2017; Berechnung und Darstellung inspire research

Auch im Vergleich zu anderen Forschungsthemen für industrielle Produktion und Technologie hat der Anteil der für IKT gewidmeten Mitteln in der Periode 2006 bis 2015 deutlich zugenommen, während der IKT-Anteil an den staatlichen Mitteln, die der Grundlagenforschung 
zur Verfügung gestellt wurden (aus der allgemeinen Hochschulfinanzierung und anderen Quellen) praktisch konstant geblieben ist.

Abbildung 47 IKT-Anteil an den staatlichen FuE-Mitteln (GBOARD) Österreichs 2006 bis 2015 für die Forschungsziele Industrielle Produktion und Technologie und Grundlagenforschung

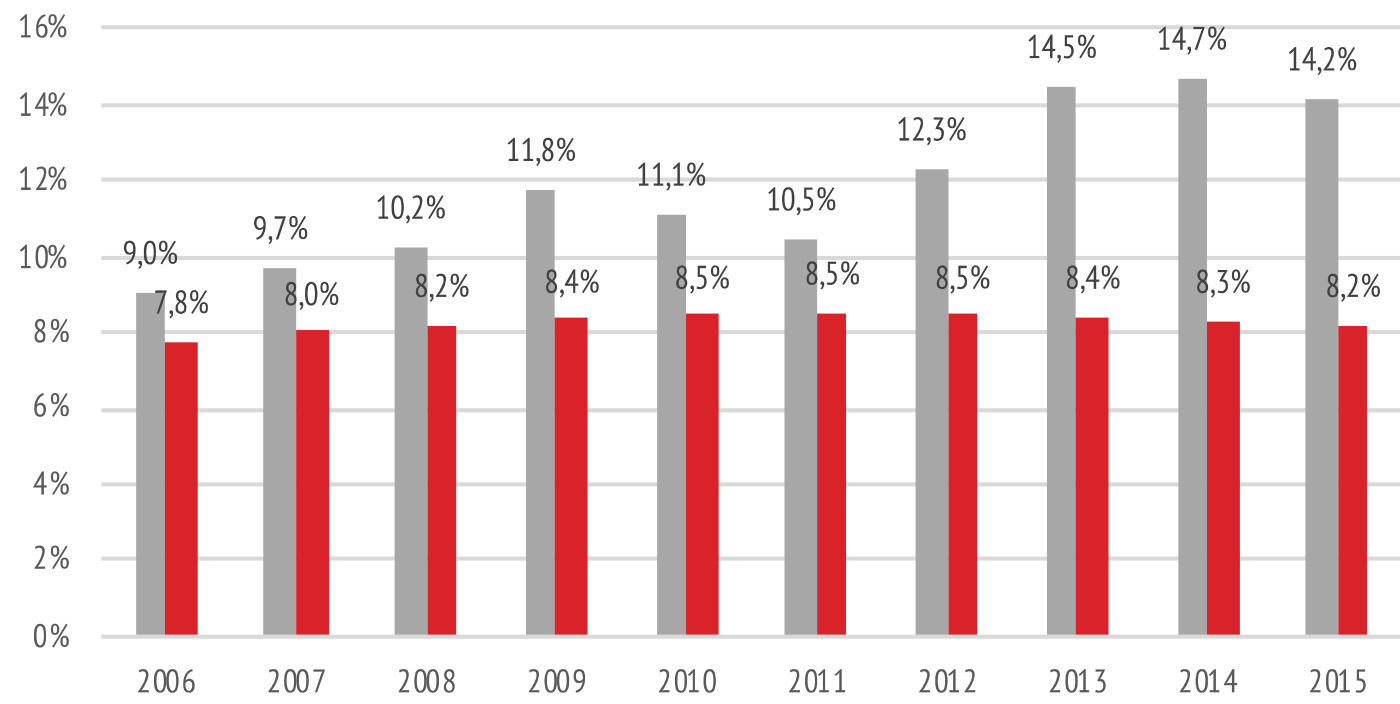

- IKT-Anteil an den staatlichen FuE-Mitteln für Industrielle Produktion und Technologie

IKT-Ante il an den staat lichen FuE-Mitteln für Grundlagenforschung (AHF und nicht-AHF)

Quelle: EC PREDICT-Datenbank 2017; Berechnung und Darstellung inspire research

In absoluten Zahlen haben die staatlichen FuE-Mittel, die Unternehmen des IKT-Sektors zugute kamen, von 27,4 Mio. Euro im Jahr 2006 auf 81,1 Mio. Euro im Jahr 2015 zugenommen.

Abbildung 48 Staatliche FuE-Mittel für Unternehmen des IKT-Sektors 2006 bis 2015 (Mio. Euro)

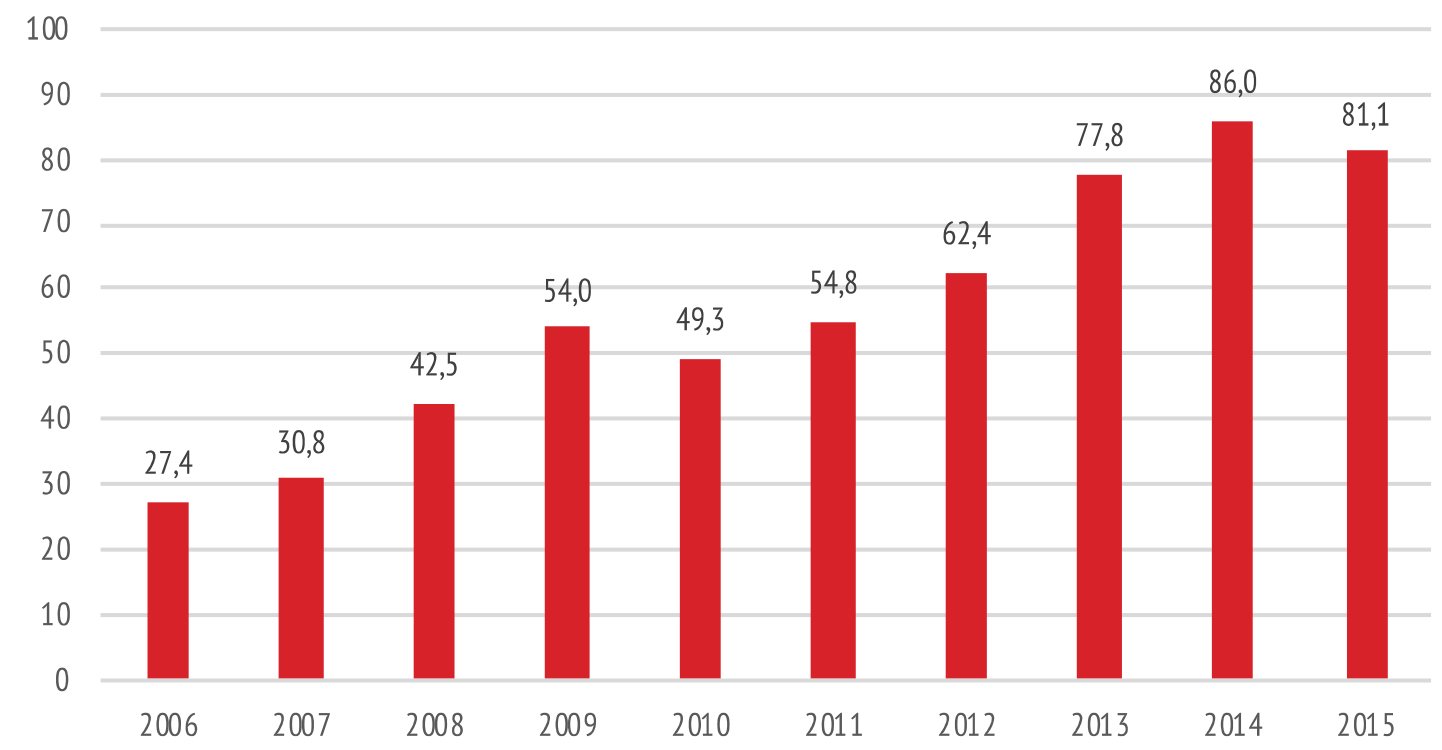

Quelle: EC PREDICT-Datenbank 2017; Berechnung und Darstellung inspire research

Damit erhöhte sich der staatliche Finanzierungsanteil an den FuE-Ausgaben der Unternehmen des IKT-Sektors von 3,5\% im Jahr 2006 auf 12,6 \% im Jahr 2015. 
Abbildung 49 Anteil der staatlichen FuE-Mittel für Unternehmen des IKT-Sektors an den gesamten FuE-Ausgaben der Unternehmen des IKT-Sektors 2006 bis 2015

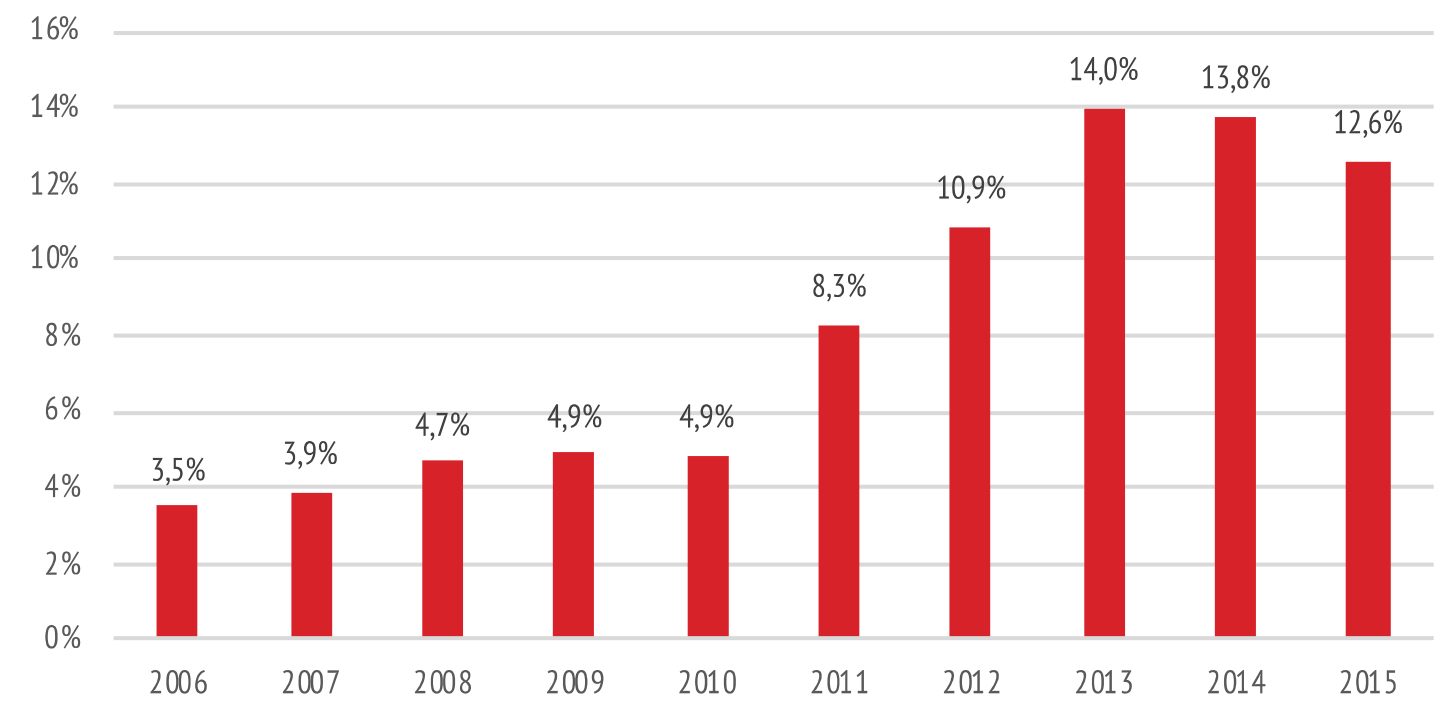

Quelle: EC PREDICT-Datenbank 2017; Berechnung und Darstellung inspire research

Mit dieser deutlichen Zunahme der öffentlichen Finanzierung der Unternehmen des IKT-Sektors geht nur zum Teil eine entsprechende Zunahme der FuE-Ausgaben der Unternehmen selbst einher. Einen sehr starken Zuwachs der Forschungsausgaben der Unternehmen hat in den Jahren 2002 bis 2016 der IKT-Dienstleistungssektor verzeichnet. Hier nahmen die FuEAusgaben von 138 Mio. Euro im Jahr 2002 auf 457 Mio. Euro im Jahr 2016 zu. Dies entspricht einer jährlichen Steigerung der Ausgaben von 8,9\%. Im Produktionsbereich des IKT-Sektors verringerten sich hingegen die FuE-Ausgaben der Unternehmen in den vergangenen 15 Jahren laut PREDICT-Datenbank von 648 Mio. Euro (2002) auf 459 Mio. Euro (2016). Dies entspricht einer jährlichen Abnahme der FuE-Ausgaben der Unternehmen von 2,4\%.

Abbildung 50 Unternehmensausgaben für FuE des IKT-Sektors 2002 bis 2016 (Mio. Euro)

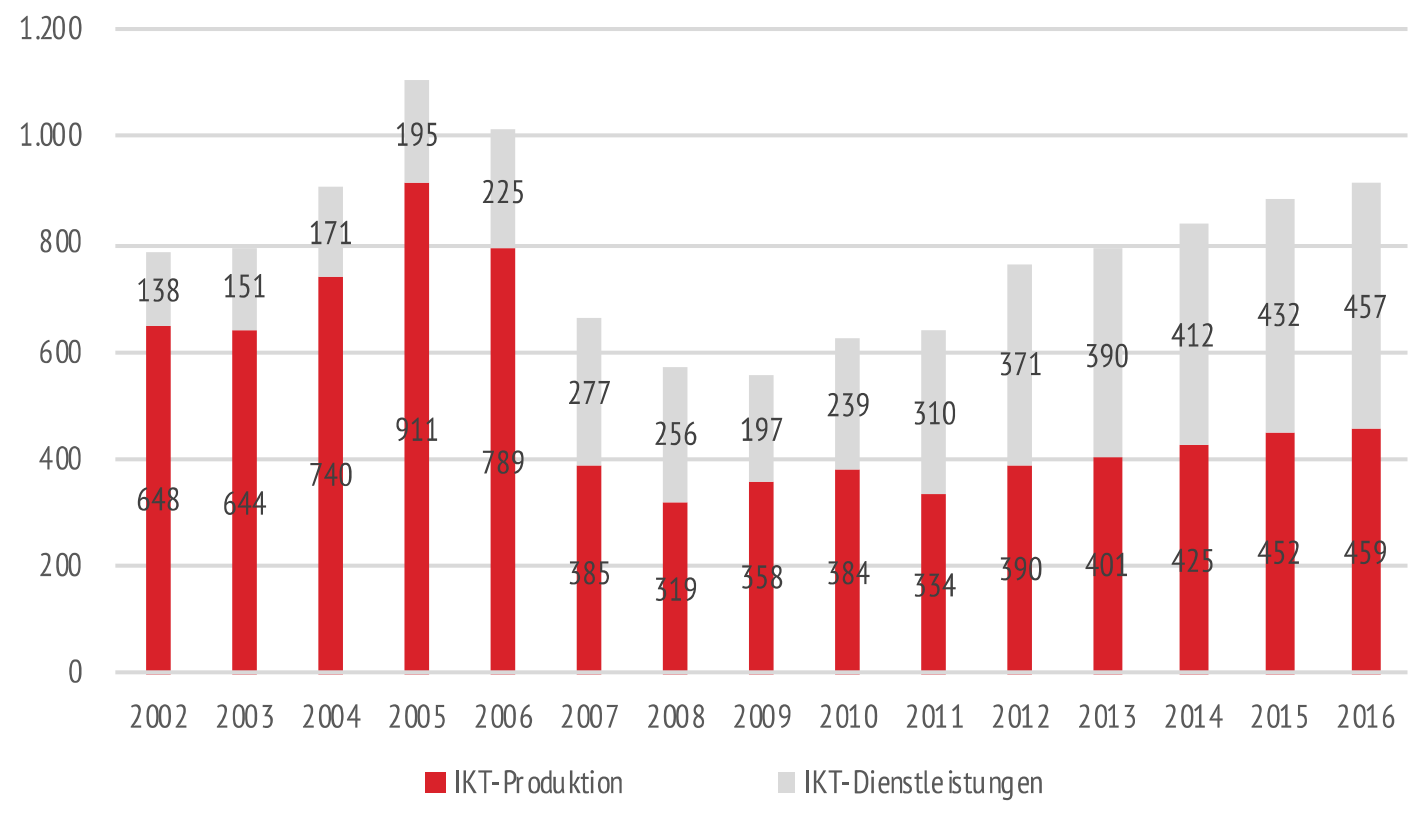

Quelle: EC PREDICT-Datenbank 2017; Berechnung und Darstellung inspire research 
Diese Gewichtsverschiebungen zwischen produzierendem Bereich und Dienstleistungsbereich des IKT-Sektors spiegeln sich auch in der Anzahl der FuE-Beschäftigten in den Unternehmen wider. Im IKT-Dienstleistungssektor verdreifachte sich zwischen 2002 und 2016 die Zahl der FuE-Beschäftigten (gemessen in Vollzeitäquivalenten) in den Unternehmen, während sich die Zahl der FuE-Beschäftigten im produzierenden Bereich des IKT-Sektors in diesem Zeitraum halbierte.

Abbildung 51 FuE-Beschäftigte in Unternehmen des IKT-Sektors 2002 bis 2016 (VZÄ)

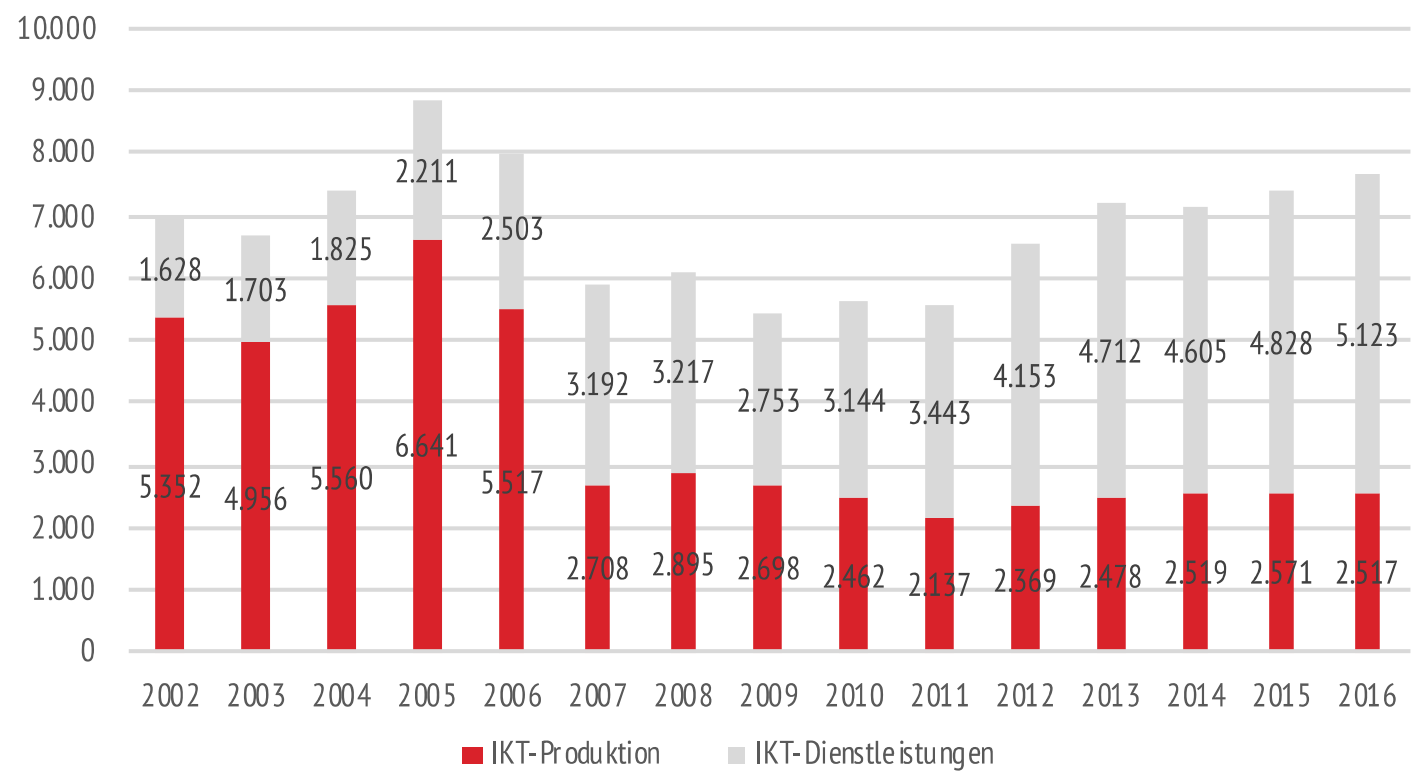

Quelle: EC PREDICT-Datenbank 2017; Berechnung und Darstellung inspire research

In Vergleich zu anderen Wirtschaftsklassen hat die Bedeutung des IKT-Sektors im engeren Sinne als Arbeitgeber für FuE-Beschäftigte in den vergangenen Jahren deutlich abgenommen. Waren im Jahr 2002 noch 26 \% aller FuE-Beschäftigten in Unternehmen im IKT-Sektor tätig, reduzierte sich dieser Anteil im Jahr 2014 auf rund $15 \%$.

Abbildung 52 Anteil der FuE-Beschäftigten in Unternehmen des IKT-Sektors an der Gesamtzahl der FuE-Beschäftigten in Unternehmen 2002 bis 2014

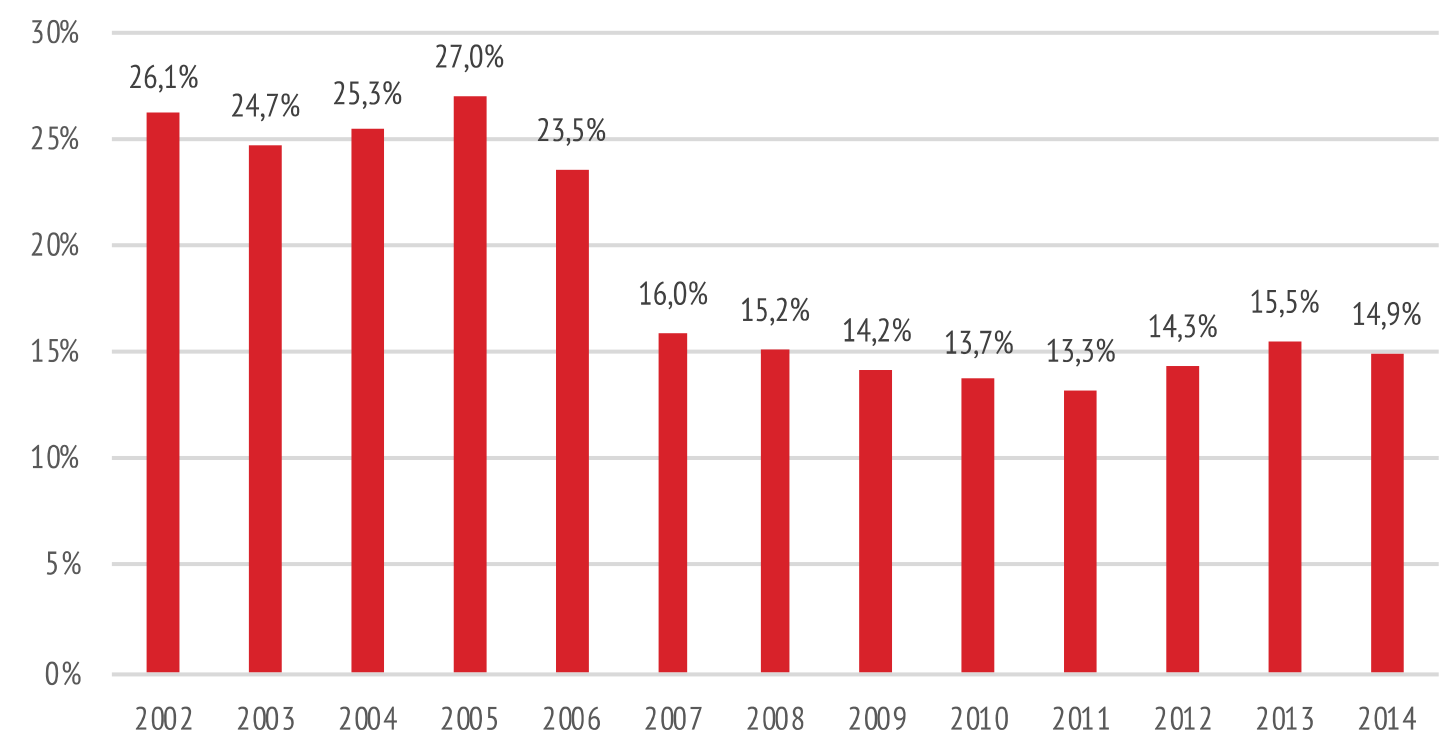

Quelle: EC PREDICT-Datenbank 2017; Berechnung und Darstellung inspire research 
Ein interessantes Detail ist, dass der Anteil des Forschungspersonals im engeren Sinne an der Zahl der FuE-Beschäftigten in den Unternehmen in den vergangenen 15 Jahren deutlich zurückgegangen ist. Waren im Jahr 2002 von 74\% der FuE-Beschäftigten in Unternehmen des IKT-Sektors Forschungspersonal, so lag dieser Anteil im Jahr 2016 nur mehr bei 61 \%. Der Anteil des FuE-unterstützenden Personal an den FuE-Beschäftigten (das sind FuE-Manager, Techniker/innen, wissenschaftliches Hilfspersonal, administratives FuE-Personal) hat hingegen entsprechend zugenommen.

Abbildung 53 Anteil des Forschungspersonals an den FuE-Beschäftigten in Unternehmen des IKTSektors 2002 bis 2016

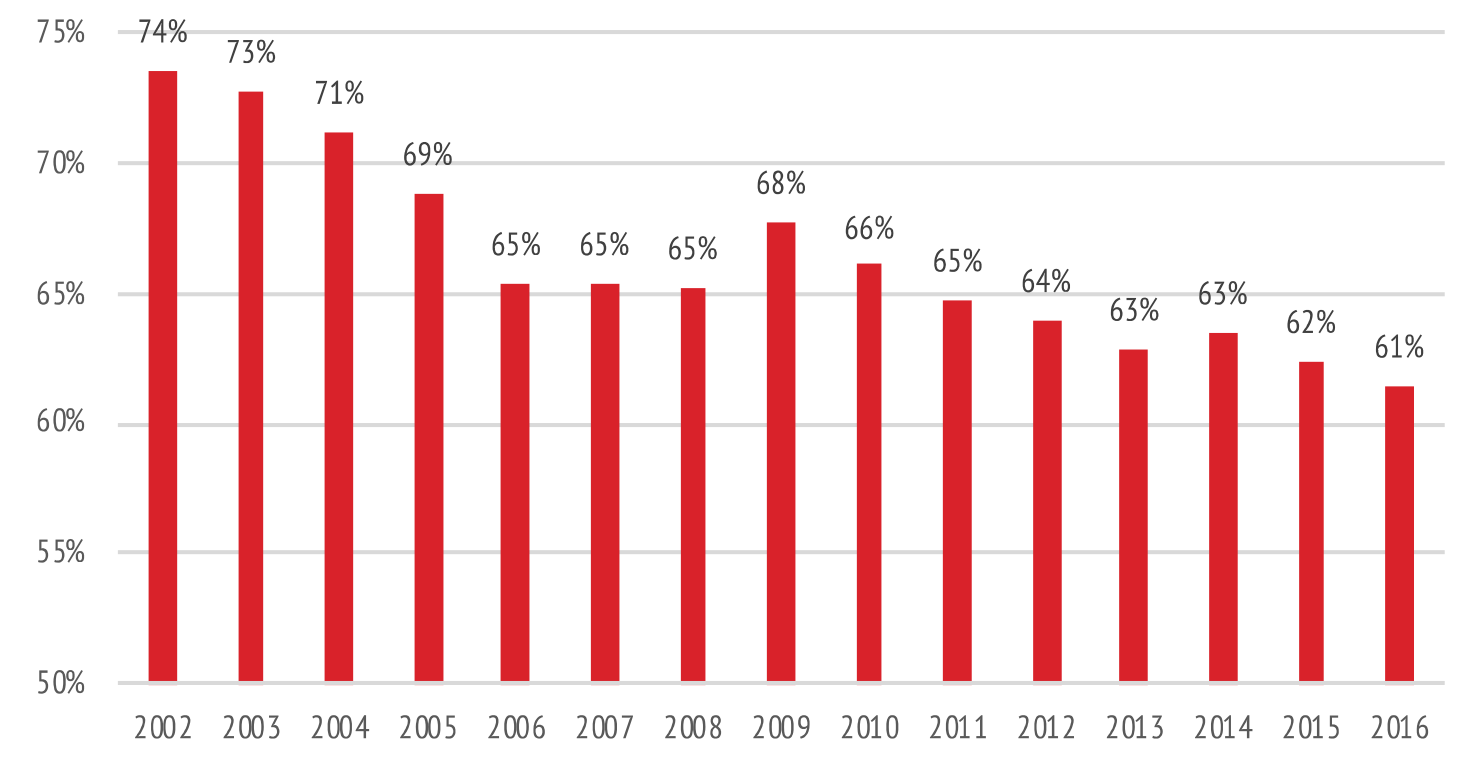

Quelle: EC PREDICT-Datenbank 2017; Berechnung und Darstellung inspire research 


\section{Anhang 2 Programmbeispiele zur IKT-Forschung in anderen Ländern}

Die in diesem Bericht skizzierten Veränderungen des Fokus bzw. der Orientierung von IKT-Forschung in Österreich sind kein nationales Phänomen. In der einen oder anderen Form zeigen sich diese Entwicklungen auch in der Forschungsförderung bzw. in den Programmprioritäten in anderen europäischen Ländern. Gemeinsam ist diesen Entwicklungen, dass es in vielen Ländern zu einer starken Ausdifferenzierung von Förderprogrammen gekommen ist, in deren Fokus IKT oder zumindest IKT-Lösungen für die zugrundeliegenden Probleme steht. Mit dem stärkeren Problem- bzw. Anwendungsbezug hat sich auch die Art der geförderten Projekte verändert. Klassische, technisch-wissenschaftlich orientierte IKT-Forschung stellt zwar nach wie vor ein Fundament auch für anwendungsorientierte Projekte dar, gleichzeitig inkludieren aber die Ziele der Projekte häufig stärker anwendungsnahe Aspekte: die FuE-Orientierung ist (zumindest teilweise) durch eine Innovationsorientierung ergänzt oder auch ersetzt worden.

In diesem Prozess verändern sich aber auch die Akteure in den Projekten und die Instrumente der Förderung. In vielen Programmen zielen die Projekte nicht mehr nur auf die Wissenschaft und den (technologieorientierten) Unternehmenssektor ab, sondern beziehen im stärkerem Umfang andere Innovationsakteure, wie Anwender/innen oder öffentliche Bedarfsträger/innen mit ein. Neben Forschung und Entwicklung spielen neue Geschäftsmodelle oder neuen Kooperationsformen / Arrangements der Leistungserbringung in den geförderten Projekten eine bedeutende Rolle.

Aus- und Weiterbildung ist ein weiterer Aspekt, der vor allem in Programmen, die sich dem Thema Digitalisierung widmen, häufig adressiert wird. Nicht zuletzt haben sich auch die Foren bzw. die organisatorische Struktur der Projekte im Fokus neuer Programme mit IKT-Bezug verändert: Neben dem klassischen (kooperativen) FuE-Projekt werden offene Entwicklungsund Innovationsansätze heute deutlich häufiger eingesetzt als noch zum Zeitpunkt des Starts des Impulsprogramms FIT-IT.

Anhand der Darstellung einzelner Programme in anderen europäischen Ländern möchten wir exemplarisch diese Entwicklungen veranschaulichen.

\section{Deutschland}

In Deutschland ist es seit 2010 aufgrund der Prominenz der Themen Digitialisierung und Industrie 4.0 zu einer starken Ausdifferenzierung des IKT-bezogenen Förderangebots gekommen. Neben dem Forschungsministerium (Bundesministerium für Bildung und Forschung BMBF) wurde dabei zunehmend auch das Wirtschaftsministerium (Bundesministerium für Wirtschaft und Energie - BMWi) als Programmanbieter aktiv. Exemplarisch für das Förderangebot in Deutschland stellen wir hier das seit 2015 laufende BMBF-Forschungsrahmenprogramm für IT-Sicherheit vor.

Bereits seit 2013 gab es zum Thema IT-Sicherheit Vorläuferaktivitäten. Parallel zu anderen bereits laufenden Förderaktivitäten zur IT-Sicherheit finanzierte das BMBF seit 2011 als zentralen Baustein der Digitalen Agenda in Deutschland zudem drei nationale Kompetenzzentren für IT-Sicherheitsforschung. Damit sollten vor allem strukturell die Forschungskapazitäten im Bereich Cybersicherheit erhöht werden. Diese Kompetenzzentren werden gemeinsam von Universitäten, Fraunhofer-Instituten und Max-Planck-Instituten an den drei Standorten Saarbrücken, Karlsruhe und Darmstadt getragen. 
Für das Forschungsprogramm des BMBF wurde in Abstimmung zwischen dem BMWi, dem Bundesinnenministerium (BMI), dem Bundesamt für Sicherheit in der Informationstechnik (BSI) und den Forschungseinrichtungen eine ressortübergreifende Agenda mit einem Zeithorizont bis 2020 erarbeitet. In diesem Rahmenprogramm wird die Forschung zur IT-Sicherheit gebündelt und auf prioritäre Themenfelder fokussiert. Diese sind:

1. Hightech für die IT-Sicherheit

2. Sichere und vertrauenswürdige IKT-Systeme

3. IT-Sicherheit in Anwendungsfeldern

4. Privatheit und der Schutz von Daten

Die Umsetzung erfolgt durch die Förderung von Verbund- oder Einzelprojekten. Als Budgetrahmen für den Zeitraum 2015 bis 2020 sind 180 Mio. Euro vorgesehen. Die bisher neun durchgeführten Ausschreibungen (Bekanntmachungen) betrafen beispielsweise die Themen ITSicherheit für Cloud Computing, Kritische Infrastrukturen, Autonomes Fahren, Anomaliererkennung / IT-Forensik und Hardware-Sicherheit. Parallel dazu können KMU über die Förderinitiative KMU-Innovativ laufend thematisch passende Projektanträge einreichen.

Eine Ausschreibung widmete sich auch dem Thema Disruptive Innovationen für Kommunikationssysteme und IT-Sicherheit. Hier erwartete sich das BMBF radikal neue Ideen und Lösungen. Dieser Anspruch konnte jedoch in der Praxis nicht erfüllt werden. Nach Einschätzung des Programmmanagements waren die überwiegende Anzahl der eingereichten Projekte im zu erwarteten Mainstream geblieben. Auch das Echo der Gutachter sei gewesen, die Ausschreibung habe kaum inhaltliche Überraschungen gebracht. Auf Basis der Erfahrungen mit der Ausschreibung stellte sich für das BMBF und das Programmmanagement die Frage, wie disruptive Innovationen effektiver gefördert werden könnten. Als Lösungsansätze hierzu wurden Aspekte identifiziert, die vor allem mit dem Forschungsprozess und den Forschungsakteuren zu tun haben und weniger mit der Form der Projektförderung. Innovationsförderlich sei es beispielsweise, vor allem junge Wissenschaftler/innen zu fördern, die vom eigenen Wissenschaftsbetrieb noch nicht vollständig geformt seien. Außerdem könne der Fördergeber durch stärker missionsorientierte Vorgaben (ähnlich DARPA Grand Challenges) das Einreichen radikal neuer Ideen unterstützen. Schließlich könne auch das Zusammenführen von Akteuren aus ganz unterschiedlichen Bereichen der Forschung und Anwendung Impulse für neue Ideen generiert.

Eine weitere Aktivität im Programm widmete sich dem Thema IT-Sicherheit für Industrie 4.0. In der Umsetzung wurde hier jedoch nicht auf die klassische kleinteilige Projektförderung im Rahmen einer Ausschreibung zurückgegriffen, sondern es wurde ein gut sichtbares nationales Referenzprojekt entwickelt. Der Vorteil dieses Ansatzes liege darin, dass ein „viel größeres Rad bewegt" werden könne, weil die relevanten Wirtschaftsverbände in das Projekt aktiv eingebunden seien und deren Strukturen und Ressourcen synergetisch genutzt werden könnten. Ein Nachteil sei jedoch gewesen, dass die unmittelbaren Bedürfnisse und Forschungsbedarfe von KMU damit nicht bedient werden hätten können.

Mit der Projektförderung im Forschungsprogramm, und hier vor allem mit der Bekanntmachung zu disruptiven Innovationen, sollten nicht nur FuE-Vorhaben im engeren Sinne, sondern auch strukturelle Verbesserungen angeregt werden, insbesondere auch für die Entwicklung der notwendigen Humanressourcen. Das Programm soll(te) dazu beitragen, Nachwuchswissenschaftler/innen (d. h. Promovierende und Postdocs) an den Hochschulen und Forschungseinrichtungen zu fördern und damit das Förderangebot der DFG Deutsche Forschungsgemeinschaft mit ihrem Fokus auf wissenschaftliche Grundlagenforschung in problem- und anwendungsorientierten Zukunftsfeldern ergänzen. 


\section{Finnland}

In Finnland haben eine hohe Anzahl der aktuell laufenden Programme der nationalen Innovations- und Standortagentur Business Finland einen Bezug zur IKT-Forschung. Digitalisierung ist eines der Hauptthemen der Business Finland Fördertätigkeit. Unter den Themenschirm Digitalisierung laufen derzeit mehrjährige FuE-Förderprogramme zu den Themen "5th Gear", "Mixed Reality“, „Al Business“, „Connectivity from Finland“, „ECOM Growth“, "Intelligent Vehicles and Mobility" und "Industrial Internet".

Als Beispiel für den Ansatz der anwendungsorientierten IKT-Forschungsförderung der letzten Jahre in Finnland greifen wir das Programm "5th Gear" heraus. Das Programm wurde 2015 von Tekes (nunmehr Business Finland) lanciert. Finnland investiert seit der Entwicklung des GSM-Standards signifikante Mittel in den FuE-Bereich Konnektivität und verfügt daher national über umfangreiches Know-how im Bereich der kabellosen Kommunikation. Das Programm 5th Gear setzt diese strategische Orientierung fort, nicht zuletzt vor dem Hintergrund einer zunehmenden globalen Konkurrenz, die aus finnischer Sicht substanzielle nationale Investitionen in Forschung und Innovation notwendig machen. Dazu komme, dass im Vergleich zu den früheren Mobilfunkgenerationen die 5G-Entwicklung stärker softwareorientiert sei, womit auch neue Akteure auftreten bzw. eingebunden werden müssten.

Das Programm 5th Gear unterstützt FuE-Projekte von finnischen Unternehmen und Forschungseinrichtungen, die Fragen der nächsten Generation der kabellosen Datenkommunikation bearbeiten. Das Programm zielt darauf ab. Finnland als führenden Standort für $5 \mathrm{G}$ zu etablieren. Auch globale Akteure sollen ihre 5G-bezogene FuE in Zukunft bevorzugt in Finnland durchführen, 5G-bezogene Investitionen tätigen und am nationalen 5G-Ökosystem teilhaben. Da die 5G-Technologie bezüglich der Anwendungsfelder viel breiter gedacht werden muss als frühere Mobilfunkstandards (z. B. Autonomes Fahren, Remote Surgery) will das Programm nicht nur die traditionellen Akteure aus dem Telekommunikationssektor erreichen, sondern auch jene in den Anwendungsfeldern, um gemeinsam technische Lösungen und neue Geschäftsmodelle zu entwickeln.

Das ursprüngliche Budget für das 5th Gear Programm betrug 100 Mio. Euro, wobei 50 \% von Tekes (Business Finland) und 50 \% von den beteiligten Unternehmen und Universitäten beigesteuert werden sollten. Wenn Programme jedoch auf hohe Nachfrage treffen, kann Business Finland seine Budgets auch umschichten. Dies war im 5th Gear Programm der Fall. Bereits bis Ende 2017 unterstützte Tekes (Business Finland) Projekte im Umfang von 170 Mio. Euro, wobei bisher rund 75 \% der Projektkosten durch Fördermittel finanziert wurden.

Das 5th Gear Programm ist ein besonders grundlagenforschungsorientiertes Programm von Business Finland. Mehr als 50 \% der Fördermittel gingen bisher an finnische Universitäten. Dies hat nach Ansicht des Programmmanagements mit den besonderen technologischen Bedingungen der 5G-Entwicklung zu tun. Der Entwicklungszyklus würde etwa zehn Jahre betragen und hohe FuE-Investitionen, gerade in die Grundlagenforschung an Universitäten, erfordern.

Obwohl das Programm stark wissenschaftlich orientiert ist, findet nur in kleinen ausgewählten Bereichen eine direkte Zusammenarbeit mit dem nationalen Wissenschaftsfonds (Academy of Finland) statt. Beide Organisationen würden jedoch derzeit in das Thema stark investieren, um so eine kritische Maße an Forschung in Finnland zu schaffen.

Mit dem 5th Gear Programm möchte Business Finland sicherstellen, dass finnische Forschung in diesem als strategisch prioritär eingeschätzten Forschungsbereich internationale Forschungsexzellenz ausweist und damit auch an globalen Netzwerken partizipieren kann. 
Dahinter steht die Überzeugung der Förderagentur, dass exzellente wissenschaftliche Forschung in den als prioritär definierten Enabling Technologies für ein kleines Land wie Finnland die Voraussetzung ist, um auch führend in Anwendungsbereichen sein zu können.

Ein zentrales Element des 5th Gear-Programms war der Aufbau von kritischer FuE-Masse durch Investitionen in vier große FuE-Testbedprojekte, die von Hochschulen und Unternehmen gemeinsam getragen werden. Die Synergien zwischen den 5 thGear-Testbeds werden genutzt, um die erforderliche Vielseitigkeit für eine gemeinsame nationale 5G-Testnetzwerkinfrastruktur zu schaffen, die eine große Vielfalt von Technologien abdeckt und den Bedürfnissen sowohl der Industrie als auch der Wissenschaft gerecht wird. An den Testbeds findet auch Forschung in den Anwendungsbereichen statt, z. B. zu den Themen Autonomes Fahren oder Smart City Infrastructure. Zusätzlich zu den vier Testbed-Projekten finanziert das Programm klassische kooperative Forschungsvorhaben mit nationaler, aber auch internationaler Orientierung.

Das Programm hat sich seit seinem Start kontinuierlich weiterentwickelt. Stand zu Beginn der Aufbau von FuE-Infrastruktur und Technologieentwicklung im Zentrum der Förderung, konzentrierte sich das Programm heute stärker auf die Förderung der vertikalen Zusammenarbeit entlang von Wertschöpfungsketten in den verschiedenen Anwendungsbereichen.

Der Programmfortschritt hat auch gezeigt, dass zu einer Reihe von Themen weiterhin Bedarf nach akademischer Grundlagenforschung besteht (z. B. Ultrareliabiltät, Low Latency). Hier würde der Wissenschaftsfonds Academy of Finland wieder ins Spiel kommen. Dieser habe über das neue Flagship-Programm die Möglichkeit, begleitend wissenschaftliche Grundlagenforschung langfristig zu fördern. Das Budget für 2018 ist dabei mit 25 Mio. Euro festgelegt. Im aktuell laufenden Flagship-Ausschreibungsverfahren hat es das Thema "6G-Enabled Wireless Smart Society \& Ecosystem“ bereits auf die Shortlist gebracht.

\section{Schweden}

Neben der FuE-Förderung in eigenen (IKT-)Programmen der schwedischen Innovationsagentur Vinnova und der verschiedenen schwedischen Wissenschaftsfonds haben sich in den vergangenen Jahren auch FuE- und Innovationsförderprogramme mit IKT-Bezug etabliert, die von mehreren Agenturen gemeinsam durchgeführt werden. Diese sind auch hinsichtlich der einbezogenen Akteure und Zielsetzungen deutlich breiter als klassische FuEProgramme aufgesetzt. Ein Beispiel dafür ist das Strategische Innovationsprogramm der schwedischen Regierung mit der Bezeichnung "Smartare Elektroniksystem (Electronic Components and Systems, ECS)", das gemeinsam von Vinnova (Innovationsagentur), Forfas (Wissenschaftsfonds für nachhaltige Entwicklung) und der Schwedischen Energieagentur finanziert wird.

Als eines von 17 Strategischen Innovationsprogrammen in Schweden setzt ECS seit 2015 die Forschungs- und Innovationsagenda "Smarter Electronic Systems for Sweden“ um. Einbezogen in die Maßnahmen des Programms sind Universitäten, Forschungsinstitute, Unternehmen und Branchenverbände. ECS ist als Öffentlich-Private-Partnerschaft eingerichtet. Die Zielgruppe der angesprochenen Akteure ist außergewöhnlich breit festgelegt. Für eine Teilnahme am Programm sind keine formalen institutionellen Voraussetzungen zu erfüllen. Jede/r, der/die zur Lösung der Herausforderungen des Programms einen Beitrag leisten kann, ist eingeladen, einen Förderantrag zu stellen. Neben dem ECS-Programm haben zumindest fünf weitere Strategische Innovationsprogramme einen klaren IKT- bzw. Digitalisierungsbezug (Drive Sweden, IfraSweden 2030, Internet of Things, ProduktionzO30 sowie IKT für Industrielle Prozesse und Automatisierung). 
Das Programmbüro wurde an das schwedische Industrieforschungsinstitut RISE Acreo (ehemals Acreo Swedish ICT) übertragen. ESC möchte Elektronische Systemtechnologien entwickeln, die in verschiedenen Branchen Anwendung findent. Dazu fördert das Programm die verstärkte Zusammenarbeit von Akteuren in Wertschöpfungsketten, trägt dazu bei, Exzellenz in Forschung und Entwicklung in Schweden aufrechtzuerhalten und setzt Maßnahmen, um genügend neue Talente im Bereich Elektronik heranzubilden.

Im Rahmen von ECS werden einerseits klassische FuE- und Innovationsprojekte (sowie Machbarkeitsstudien) finanziert. Die Fördermittel für die Projektförderung sind jedoch vergleichsweise bescheiden. Im Jahr 2017 wurden beispielsweise Projekte mit einem Volumen von 95,2 Mio. SEK (9,5 Mio. Euro) im Umfang von 44,8 Mio. SEK (4,5 Mio Euro) gefördert. Die Ausschreibungen im ECS Programm laufen dabei über die finanzierenden Förderagenturen VINNOVA, die Schwedische Energieagentur und FORMAS. Das ESC Programmmanagement tritt nicht als finanzierende Programmagentur auf, ist jedoch in die Formulierung der Ausschreibungen mit einbezogen.

Neben der FuE-Projektförderung setzt ESC weitere Maßnahmen. Beispielsweise wurden für die Technologiefelder Photonik, Embedded Systems, Mikro- und Nanoelektronik, Power Electronics, Integrated Sensor Systems for Health, Integrated Circuits and Systems, Construction Set - Cross Connect, Printed Electronics und Reliable Electronics Hardware sogenannte "Kompetensnavs" (Kompetenzhubs) aufgebaut. Kompetenzhubs fungieren als Plattformen, über die der Austausch zwischen Unternehmen, Universitäten und Forschungsinstituten für Innovationsprojekte organisiert wird. In einer weiteren Programmlinie mit der Bezeichnung "Kompetensförsörjning" (Kompetenzen aufbauen) arbeitet ECS mit Schulen und Bildungseinrichtungen zusammen, um Lehr- und Lernmaterialen zum Thema Elektronik bereitzustellen. ECS ist auch im Beirat vertreten, der für die Entwicklung der Lehrpläne im Sekundarschulbereich verantwortlich ist. Ziel ist es, Elektronik als Fach in den schwedischen Gymnasien einzuführen.

Für die kommenden Jahre hat das ESC-Büro von den drei Förderagenturen den Auftrag erhalten, auch international besser sichtbar zu werden. Ziel ist es, die verschiedenen vorhandenen Finanzierungsquellen (z. B. H2020, Strukturfonds und nationale Förderungen) für Innovationen zu Smart Electronic Systems effektiver zu kombinieren. Schwedische Unternehmen sollen verstärkt zur Zusammenarbeit mit Unternehmen und Universitäten in anderen europäischen Ländern angeregt werden. Derzeit besteht die Schnittstelle von ESC zur Gemeinsamen Technologieinitiative ECSEL ausschließlich darin, dass Mitglieder des ECS-Steuerungsgremium in Unternehmen und Forschungseinrichtungen arbeiten, die bereits auch an ECSEL-Projekten teilgenommen haben. Eine Verzahnung von nationaler und europäischer Agenda besteht jedoch derzeit nicht.

Programmorientierte FuE-Projektförderung im Bereich der angewandten problem- bzw. anwendungsfeldorientierten wissenschaftlichen Forschung hat in Schweden in den vergangenen Jahren vor allem auch die Schwedische Agentur für Strategische Forschung (Swedish Foundation for Strategic Research) gefördert. Aktuell startet beispielsweise ein mit 300 Mio. SEK (30 Mio. Euro) dotiertes Programm zum Thema "Cybersecurity and Information Security“. Gefördert werden kollaborative interdisziplinäre FuE-Projekte von Forschungsgruppen für einen Zeitraum von fünf Jahren, wobei die jährliche Förderung der Forschungsgruppen an den antragsberechtigen Hochschulen und außeruniversitären Forschungseinrichtungen zwischen 4 Mio. SEK (400 Tsd. Euro) und 7 Mio. SEK (700 Tsd. Euro) betragen kann.

Das Programm ist abgestimmt mit einem anderen der insgesamt 17 Strategischen Innovationsprogramme der schwedischen Regierung (siehe oben) zum Thema "Connected Industry". 
Seit 2014 wurden mit dem Programm "Rambidrag Electronics" weitere 250 Mio. SEK (25 Mio. Euro) für Projekte zu den Themen "Post CMOS", "More than Moore-Elektronik" sowie "Techniques for high data-rate communications" entlang der selben Förderkriterien vergeben. Weitere 100 Mio. SEK (10 Mio. Euro) wurden seit 2014 für FuE-Infrastrukturmaßnahmen an den Universitäten für die IKT-Forschung im Programm "Research Infrastructure Fellows" aufgewendet. Schließlich läuft seit 2016 das ebenfalls mit 300 Mio. SEK (30 Mio. Euro) dotierte Programm "Smart Systems" mit den Förderthemen "Cyber Physical Systems", "Integrated Systems", "Systems of Systems", "Automation", „Autonomous Systems" und "Artificial Intelligence-Based Informations System".

\section{Niederlande}

Neben der klassischen, projektbezogenen FuE-Forschungsförderung an Universitäten und außeruniversitären Forschungseinrichtungen, auch in Kooperation mit Unternehmen, haben sich in den Niederlanden in den letzten Jahren neue Programmformate entwickelt. Ein Bespiel dafür ist das Partnerschaftsprogramm zwischen der ehemaligen Förderagentur STW (nunmehr als TTW ein Teil von NWO - Netherlands Organisation for Scientific Research) und dem Unternehmen NXP mit der Bezeichnung "Advanced 5G Solutions". Mit dem für 2017 bis 2021 eingerichteten Programm sollen neue Lösungen und Durchbrüche mit dem technischen Fokus auf "Advanced IC \& Antenna Design“, "Signal Processing" und "System Research" für Anwendungen in der Kommunikationstechnik und für den Automotiv-Bereich erarbeitet werden.

Mit dem Finanzierungsinstrument "Partnerschaften“ sollen Fragen und aktuelle Herausforderungen aus der Wirtschaft gemeinsam mit den niederländischen Universitäten und Forschungseinrichtungen bearbeitet und gelöst werden. Kern einer Partnerschaft ist eine Themenbeschreibung, die von einem einzelnen oder mehreren Unternehmen gemeinsam verfasst wird. NWO TTW organisiert auf Basis dieser Themenbeschreibung eine Ausschreibung, an der sich Wissenschaftler/innen an Universitäten und Forschungseinrichtungen beteiligen können, um Ideen für Lösungen der aufgeworfenen Forschungsfragen in den Themenbeschreibungen vorzuschlagen. Die Ausschreibungen dienen also einer Art "matchmaking" zwischen Wirtschaft und qualifizierten Forscher/inne/n. Die einlangenden Projektvorschläge werden gemäß dem Standardverfahren von NWO TWW wissenschaftlich begutachtet und gereiht. Die Auswahl der zur Förderung gelangenden Projekte nimmt das zuständige TTWGremium vor. Ein Programmausschuss aus Mitgliedern der Unternehmen und von Wissenschaftler/innen, die durch NWO TTW nominiert werden, begleiten die Abwicklung des Programms.

Die Projekte des Forschungsprogramms der Partnerschaft werden durch NWO TWW im Ausmaß von 50 \% der bei den Forschungspartnern anfallenden Kosten finanziert. Das Mindestprojektvolumen einer Partnerschaft ist mit 3 Mio. Euro festgelegt. Den Unternehmen wird über das Ausschreibungsverfahren Zugang zu den jeweils am besten geeigneten Wissenschaftler/innen in den Niederlanden gegeben. Ein Einstieg anderer Partner in das Programm ist nicht vorgesehen. Ähnliche Partnerschaftsprogramme mit IKT-Bezug bestehen derzeit in den Niederlanden mit den Unternehmen Bronkhorst High Tech, KROHNE Altometer sowie Philips.

Ein weiterer, nicht klassisch FuE-Projekt-bezogener Programmansatz zur Förderung von innovativen IKT-Lösungen in Anwendungskontexten in den Niederlanden ist das Smart Industry Action Programme. Dieses wurde entwickelt, nachdem in Deutschland das Zu- 
kunftsprojekt Industrie 4.0 gestartet wurde. Die Niederlande wollten eine vergleichbare Initiativen setzen: ein Vorhaben, das von der Wirtschaft und den relevanten Ministerien auch sehr positiv aufgenommen und unmittelbar aufgegriffen wurde. Es wurde daraufhin ein nationaler Konsultationsprozess gestartet, der alle wichtigen Stakeholder, insbesondere aus der Wirtschaft, mit einbezog. Die deutsche Industrie 4.0-Initiative sei laut den niederländischen Programmverantwortlichen stark von großen IT-Unternehmen getragen, während KMU in die Programmmaßnahmen wenig eingebunden seien. Das niederländische Aktionsprogramm Smart Industry sollte hier breiter aufgesetzt sein.

Am Smart Industry Action Programm sind das niederländische Wirtschaftsministerium, die Arbeitgeberverbände, die Wirtschaftskammer, Universitäten und TNO als national führende, anwendungsorientierte Forschungseinrichtung beteiligt. Smart Industry wird von einem Steuerungsgremium geführt, dem ein Programmbüro beigestellt ist, das bei TNO angesiedelt ist. Jede Trägereinrichtung finanziert eine Person des Programmbüros im Ausmaß von jeweils 0,8 Vollzeitäquivalenten. Das Smart Industry Action Programme verfügt über keine eigenen Mittel zur Forschungsfinanzierung.

Übergreifendes Ziel des Programms ist die Entwicklung intelligenter IKT-Lösungen, um Operationen von Maschinen zu vernetzen, nicht nur innerhalb von Unternehmen, sondern auch zwischen Unternehmen und zwischen Unternehmen und ihren Kunden. Das Programm setzt drei Aktionslinien um: 1) Awareness, 2) Field Labs und 3) Stärken der Grundlagen für Innovationen.

In der Aktionslinie Awareness werden vor allem Präsentationen und Workshops organisiert. Rund 200 Smart Industry-Botschafter/innen helfen mit, das Thema in der niederländischen Wirtschaft zu verbreiten. Zielgruppe sind vor allem KMU und interessierte Einzelpersonen aus dem Unternehmensbereich. Jährlich werden mit dieser Maßnahme rund 10.000 Personen erreicht. Daneben wurden in der Aktionslinie Awareness Online-Ausbildungsmodule und Angebote für Team-Trainings entwickelt.

In der ersten Programmperiode 2014 bis 2017 wurde die Aktionslinie Field Labs sehr intensiv verfolgt. Field Labs sind praxisorientierte Umgebungen, die es Unternehmen, Hochschulen und Forschungsorganisationen erlauben, Smart Industry-Lösungen zu entwickeln und zu testen. Field Labs werden in einem realen industriellen Umfeld eingerichtet. Neben Entwicklungsaufgaben haben zahlreiche Fieldlabs auch einen Fokus auf Aus- und Weiterbildung und arbeiten dabei mit Schulen und Einrichtungen der beruflichen Bildung zusammen. Etwa die Hälfte der aktuell 29 Field Labs verfolgen Ausbildungsschwerpunkte. Die Teilnehmer/innen sollen lernen, mit Smart Industry-Lösungen praktisch zu arbeiten. Field Labs entwickeln sich häufig bottom-up entlang einer Wertschöpfungskette in einem regionalen Ökosystem, wobei aber auch Hochschulen und Forschungsorganisationen eingebunden sind. Field Labs dienen dazu, Forscher/innen aus ihren Labors heraus ins das industrielle Feld zu bringen.

Finanziert werden die Field Labs durch die Sachleistungen der beteiligten Einrichtungen sowie durch Finanzierungsbeiträge der öffentlichen Hand. Organisatorisch können Field Labs als Stiftungen, als Non-Profit-Organisationen oder ohne gesonderte institutionelle Struktur über Kooperationsverträge eingerichtet sein. Das Wirtschaftsministerium stellte dafür insgesamt 15 Mio. Euro an Fördermittel zur Verfügung.

Die Aktionslinie "Stärken der Grundlagen für Innovationen“ umfasst Maßnahmen, die auch in klassischen FuE-Förderprogrammen angetroffen werden. Zur Unterstützung der Aktionslinie konnten von den niederländischen Forschungsförderorganisationen NWO (Netherlands Organisation for Scientific Research) und der Technologiestiftung STW (heute ebenfalls Teil von 
NWO) FuE-Themen ausgeschrieben werden. Eine weitere eher klassische Maßnahme in dieser Programmlinie war die Ausarbeitung einer langfristigen Forschungsagenda, die in Zusammenarbeit zwischen den niederländischen "Topsektoren", den Universitäten und den Forschungsförderagenturen erstellt wurde.

Das Smart Industry Action Programm widmet sich dezidiert auch Fragen der Qualifizierung von Beschäftigten im Zusammenhang mit der industriellen Digitalisierung und arbeitet an der Weiterentwicklung von Curricula und Ausbildungsplänen mit. Schließlich gibt es noch Maßnahmen, die sich mit Forschungs- und Innovationsfragen zu Big Data, Cyber Security und Software für Smart Industry beschäftigen.

\section{Fazit aus den internationalen Beispielen}

Aus der Darstellung der Entwicklungen in anderen europäischen Ländern wird deutlich, dass sich in den vergangenen Jahren sowohl die Ausrichtung der Programme als auch die Akteurslandschaft in den IKT-Förderprogrammen deutlich verbreitert hat. Getrieben durch das Thema Digitalisierung sind die Grenzen zwischen dem IKT-Bereich im engeren Sinne und den Anwendungsbereichen immer fluider geworden bzw. ineinander verschmolzen. Dies hatte nicht nur eine Auswirkung auf Inhalte und Schwerpunkte der Forschung, sondern auch auf die Akteure, die mit den Programmen adressiert werden.

Auch die Orte für die Programmsteuerung und das Programmmanagement haben sich damit geändert: Das schwedische ECS-Programm zur Umsetzung der Innovationsagenda für "Smarter Electronic Systems" wird von einem Industrieforschungsinstitut getragen. Am Programm beteiligt sind Akteure aus Universitäten, Forschungsinstituten, Branchenverbänden und Unternehmen. Die öffentliche Finanzierung stammt von drei unterschiedlichen Förderagenturen (Vinnova, Forfas und Schwedische Energieagentur). In das niederländische Smart Industry Programm sind nicht nur im Programmdesign, sondern auch in der Abwicklung neben dem Wirtschaftsministerium Arbeitgeberverbände, Wirtschaftskammern, Universitäten und die nationale außeruniversitäre Forschungseinrichtung TNO, welche auch das Programmbüro stellt, involviert. Nur das finnische 5 th Gear-Programm, das von Business Finland getragen und abgewickelt, ist noch traditionell als klassisches Forschungsprogramm strukturiert. Die breite Involvierung von verschiedenen Akteuren spiegelt damit vor allem auch die Breite und Dringlichkeit des Themas Digitalisierung wider.

Das bedeutet aber nicht, dass klassische IKT-Grundlagenforschung in diesen Ländern verschwunden wäre. Im Gegenteil: Neben der problem- und anwendungsfeldbezogenen Forschung wird in anderen Ländern zunehmend wieder Wert auf die akademische Grundlagenforschung im IKT-Bereich gelegt und über Instrumente der Forschungsförderung an Hochschulen auch substanziell finanziert.

Die Themen Bildung und Ausbildung haben jedenfalls an Bedeutung stark gewonnen, womit sowohl der akademische Nachwuchs, als auch die Aus- und Weiterbildung im Unternehmensbereich und Maßnahmen, um IKT und Digitalisierungsthemen in der Sekundarstufe von Schulen attraktiver zu machen, gemeint sein können. Das schwedische ECS-Programm entwickelt beispielweise Lehr- und Lernmaterialen zum Thema Elektronik und verteilt diese über bestehenden Bildungsnetzwerke an Schulen in ganz Schweden. Daneben gibt es derzeit Bestrebungen, Elektronik als Fach in der Sekundarstufe (Gymnasium) einzuführen, und ECS-Vertreter arbeiten an der Entwicklung von Lehrplänen in den zuständigen Gremien mit. Die niederländischen Field Labs wiederum waren zwar ursprünglich als angewandte FuEEinrichtungen konzipiert, haben sich aber in der Praxis immer stärker um das Thema Ausund Weiterbildung gekümmert. Etwa die Hälfte der derzeit bestehenden Field Labs haben 
einen Fokus auf Ausbildung, arbeiten mit (Berufs)schulen zusammen oder bieten Schulungen für Studierende und Berufstätige in Unternehmen an. In Deutschland ist zwar aus zuwendungsrechtlichen Gründen fördertechnisch nur die Förderung von (FuE-)Projekten möglich. Aber auch in den IKT-Programmen Deutschland ist zu beobachten, wie die beteiligten Ministerien und Projektträger im Rahmen der rechtlichen Möglichkeiten mit neuen Förderansätzen zu den Themen Ausbildung und Qualifizierung experimentieren, um die FuE-Projektförderung für IKT-Themen insgesamt treffsicherer und effektiver zu machen. 


\section{Anhang 3 Online-Befragungen der Förderempfänger}

Grundgesamtheit für die Adressaten der Befragung waren die in der FFG-Datenbank angeführten technischen Ansprechpersonen bewilligter Anträge in den BMVIT IKT-Programmen seit Start des Impulsprogramms FIT-IT. Um Doppelbefragungen zu vermeiden, wurde bei Personen, die in mehreren geförderten Projekten als technische Ansprechperson angeführt sind, jeweils nur das erste bewilligte Projekt für die Befragung berücksichtigt. Außerdem wurden alle technischen Ansprechpersonen bzw. die innen zugeordneten Projekte ausgeschieden, die sich bereits im Jahr 2015 an der Online-Befragung der Evaluierung der österreichischen Beteiligung am Ambient and Assisted Living Joint Programme beteiligt hatten. Schließlich wurden für die Befragung alle Projekte ausgeschieden, die im Jahr 2013 abgeschlossen wurden, da diese Gruppe von Adressaten im Herbst 2017 einen Fragebogen zum Erfolg der Projektförderung im Rahmen des FFG-Wirkungsmonitorings erhielten. Dieses Verfahren lieferte eine Stichprobe von 660 Personen, für die in der FFG-Datenbank eine Kontakt-E-MailAdresse hinterlegt ist.

Tabelle 31 Kennzahlen zur Online-Befragung der technischen Ansprechpersonen der Projektpartner in geförderten Projekten der BMVIT IKT-Programme 2002 bis 2016

\begin{tabular}{|c|c|c|c|}
\hline Kennzahlen zur Teilnehmerbefragung & $\begin{array}{l}\text { Ex-Post- } \\
\text { Befragung }\end{array}$ & $\begin{array}{l}\text { Interims- } \\
\text { Befragung }\end{array}$ & Gesamt \\
\hline Förderfälle gesamt & 1.254 & 764 & 2.018 \\
\hline Stichprobe & 660 & 381 & 1.041 \\
\hline darunter mit gültiger E-Mail-Adresse & 444 & 323 & 767 \\
\hline Verwertbarer Rücklauf & 240 & 225 & 465 \\
\hline in \% der gültigen E-Mail-Adressen & $54,1 \%$ & $69,7 \%$ & $60,6 \%$ \\
\hline in \% der Stichprobe & $36,4 \%$ & $59,1 \%$ & $44.7 \%$ \\
\hline in \% der Förderfälle & $19,1 \%$ & $29,5 \%$ & $23,0 \%$ \\
\hline \multicolumn{4}{|l|}{ Verwertbarer Rücklauf } \\
\hline unter Unternehmen in \% der gültigen E-Mail-Adressen & $48,9 \%$ & $67,8 \%$ & $57,2 \%$ \\
\hline $\begin{array}{l}\text { Verwertbarer Rücklauf unter wissenschaftlichen } \\
\text { Einrichtungen in \% der gültigen E-Mail-Adressen }\end{array}$ & $59.7 \%$ & $72,1 \%$ & $64.7 \%$ \\
\hline $\begin{array}{l}\text { unter Projektpartnern in Demographischer Wandel- } \\
\text { Projekten in \% der gültigen E-Mail-Adressen }\end{array}$ & $53,8 \%$ & $70,2 \%$ & $62,2 \%$ \\
\hline $\begin{array}{l}\text { unter Projektpartnern in FIT-IT/IKTdZ-Projekten in \% der } \\
\text { gültigen E-Mail-Adressen }\end{array}$ & $53,1 \%$ & $70,4 \%$ & $59,1 \%$ \\
\hline $\begin{array}{l}\text { unter Projektpartnern in JTI-Programmen in \% der gültigen } \\
\text { E-Mail-Adressen }\end{array}$ & $61,4 \%$ & $67,1 \%$ & $64.9 \%$ \\
\hline
\end{tabular}

Quelle: Befragung Evaluierung BMVIT IKT-Programme 2017; Berechnung inspire research

Die Zuordnung der Adressaten zu den beiden entwickelten Fragebögen für die Ex-post-Evaluierung bzw. die Interimsevaluierung wurde nicht streng nach der Zuordnung der Projekte zu Ausschreibungen in den Programmen FIT-IT bzW. IKT der Zukunft vorgenommen, sondern nach dem Zeitpunkt des Projektabschlusses. Projekte (bzw. die technischen Ansprechpersonen dieser Projekte), die bis zum 31. Dezember 2014 abgeschlossen wurden, erhielten den Fragebogen für die Ex-Post-Befragung, später abgeschlossene bzw. alle laufenden Projekte erhielten den Fragebogen für die Interims-Befragung. 


\section{Anhang 4 Quellenverzeichnis}

Bundesministerium für Wissenschaft und Forschung, Bundesministerium für Verkehr, Innovation und Technologie, Bundesministerium für Wirtschaft und Arbeit, 2007, Österreichischer Forschungs- und Technologiebericht 2007. Lagebericht gem. § 8 (1) FOG über die aus Bundesmitteln geförderte Forschung. Technologie und Innovation in Österreich. Wien.

ECSEL Joint Undertaking, 2015, Financial Transparency Reporting. Recipients of ECSEL JU Grans for Research Actions, Calls 2014. Brussels.

ECSEL Joint Undertaking, 2016, List of Recipients 2015. Brussels.

ECSEL Joint Undertaking, 2017, List of Recipients 2016. Brussels.

European Commission, 2017, eurostat, Share of Member States in EU GDP (online: http://ec.europa.eu/eurostat/web/products-eurostat-news/-/DDN-20170410-1).

European Commission, 2017, 2017 PREDICT Dataset (online: https://ec.europa.eu/jrc/en/predict/ict-sector-analysis-2017/data-metadata).

European Parliament, 2015, EU budget management: innovation agency and ITER project fail 2013 discharge test. Press release. 23.03.2015. Ref.: 20150323IPR36410.

European Parliament, 2016, U budget management: ARTEMIS, ENIAC and ITER projects fail 2014 discharge test. Press release. 04.04.2016. Ref.: 20160404IPR21395.

Geyer, Anton und Barbara Good, 2016, Evaluierung der österreichischen Beteiligung am Ambient Assisted Living Joint Programme (AAL JP 2008 - 2013). Technopolis. Wien.

Geyer, Anton, 2012, Interimsevaluierung der FIT-IT Initiativen ARTEMIS und ENIAC. Endbericht. Technopolis. Wien.

Kaufmann, Peter und Elisabeth Nindl, 2018, Wirkungsmonitoring der FFG Förderung 2017. Studie im Auftrag der FFG Österreichische Forschungsförderungsgesellschaft. Wien.

Österreichische Forschungsförderungsgesellschaft FFG, 2011, Forschungserfolge. Der Jahresbericht der Österreichischen Forschungsförderungsgesellschaft FFG, Mittwoch, 6. April 2017, Wien.

Österreichische Forschungsförderungsgesellschaft FFG, 2017, Forschungserfolge. Der Jahresbericht der Österreichischen Forschungsförderungsgesellschaft FFG, Mittwoch, 5. April 2011, Wien.

Statistik Austria, 2018, FIT-IT Sonderauswertung der FuE-Erhebungen 2004, 2009 und 2013. Unveröffentlicht. Wien.

Thompson, Haydn, Emilio Lora-Tamayo, Werner Damm, Jean-Luc Dormoy, Leonard Hobbs, Margriet Jansz, Tomasz Kosmider und Wolfgang Pribyl. 2017, Interim Evaluation of the ECSEL Joint Undertaking (2014-2016) Operating under Horizon 2020. Final Report. doi: $10.2759 / 614017$

Uusikylä, Petri, Carmen Ceinos Kohn, Agnieszka Ciesla, Anton Geyer, Heidrun Mollenkopf und Michael Sharpe, 2017. Interim Evaluation of the Active \& Assisted Living Programme. October 2017. European Commission. Brussels. doi: 10.2759/785852

Zinöcker, Klaus, Julia Schindler, Wolfgang Polt, Stefanie Gude, Michael Stampfer und Julia Schmidmayer, 2005. FIT-IT Interimsevaluierung. Konzepte, Rahmenbedingungen, Design, Prozesse. Snapshots auf Wirkung und Additionalität. Joanneum Research. Wien. 
inspireresearch

inspire research Beratungsgesellschaft m.b.H.

Lange Gasse 65/16, A-1080 Vienna, Austria

office@inspire-research.at

www.inspire-research.at 